\title{
Ursachen früher gesundheitlicher Ungleichheiten und ihre schulischen Folgen
}

\author{
Dissertation \\ zur Erlangung des Doktorgrades \\ der Sozialwissenschaftlichen Fakultät \\ der Georg-August-Universität Göttingen
}

vorgelegt von

Sten Becker

(geboren in Cottbus)

Göttingen, März 2018 


\section{Betreuungsausschuss}

Erstbetreuerin: $\quad$ Prof. Dr. Karin Kurz

Weitere Betreuer: $\quad$ Prof. Dr. Thorsten Schneider und Prof. Dr. Steffen Kühnel

Tag der mündlichen Prüfung: 21.12.2017 


\section{Danksagung}

Die vorliegende Dissertation entstand während meiner Mitarbeit in Forschung und Lehre an den Universitäten in Göttingen, Potsdam und Halle (Saale).

Prof. Dr. Karin Kurz gilt mein herzlicher Dank für die Möglichkeit zur Promotion und die Bestärkung darin, meinen Forschungsinteressen an der Schnittstelle Gesundheit/Bildung zu folgen. Bedanken möchte ich mich auch für die gute und angenehme Zusammenarbeit.

Auch bei Prof. Dr. Thorsten Schneider und Prof. Dr. Steffen Kühnel möchte ich mich herzlich bedanken. Beide erklärten sich zu einer bereits fortgeschrittenen Phase der Promotion bereit, den Betreuungsausschuss zu vervollständigen. Als Zweit- bzw. Drittgutachter gaben sie mir wichtige inhaltliche und methodische Hinweise.

Für fachlich offene Diskussionen in und außerhalb des Instituts bedanke ich mich bei meinen Göttinger Arbeitskolleginnen und Kollegen, besonders bei Carolyn Stolberg, Sabine Keller, Jörg Hartmann, Diana Schacht, Paul Schmelzer und Kerstin Schulze. Regina Wandrey-Traudt möchte ich danken für ermutigenden Zuspruch und den unerschöpflichen Vorrat an Süßem, zu dem selbst zu Schließzeiten des Sekretariats Zugang bestand.

Für wertvolle Rückmeldung zu Kapitel 7 danke ich Prof. Dr. Wolfgang Lauterbach und meinen Potsdamer Kolleginnen und Kollegen am Lehrstuhl für Sozialwissenschaftliche Bildungsforschung.

Prof. Dr. Oliver Arránz Becker und dem ganzen Methodenteam am Institut für Soziologie der Martin-Luther-Universität Halle-Wittenberg gebührt mein ausdrücklicher Dank für hilfreiche Hinweise. Besonders danken möchte ich meinen Kolleginnen Vivian Herold, Katharina Loter, Eva-Maria Schultze und Audrey Terracher-Lipinski, für Feedback, Motivation und Energie, welche ich aus der gemeinsamen Zeit für den Endspurt schöpfte.

Während der gesamten Promotionszeit wurde mir der uneingeschränkte Rückhalt meiner Familie zuteil: Eveline, Hans-Jürgen und Paweł - danke! 


\section{Inhalt}

$1 \quad$ Einleitung........................................................................................................................ 4

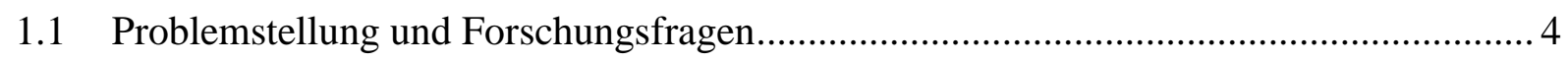

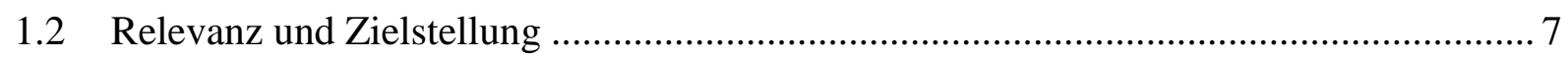

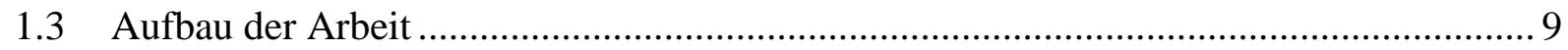

2 Theoretische Bezüge und Befunde zu sozialen Chancenungleichheiten ................... 11

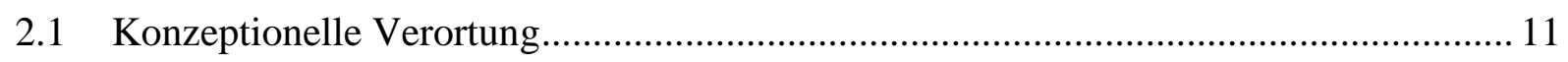

2.1.1 Statuserwerbsforschung - Ungleichheit als mehrstufiger Prozess ............................... 11

2.1.2 Humankapitaltheorie - Bildung und Gesundheit als Investition ................................. 13

2.1.3 Theorie sozialer Produktionsfaktoren - Bildung und Gesundheit als Bedürfnis............ 14

2.2 Boudon's mikrosoziologischer Erklärungsansatz.................................................... 15

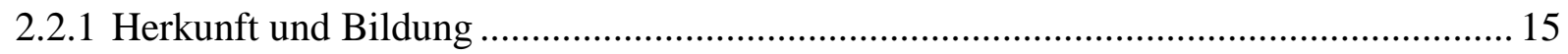

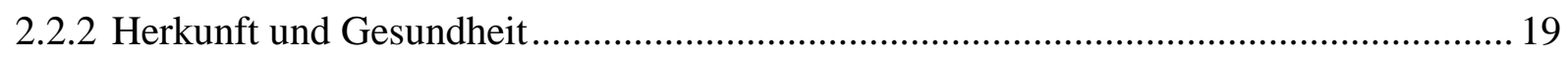

2.3 Bourdieus gesellschaftstheoretischer Erklärungsansatz …........................................ 22

2.3.1 Bourdieus analytischer Zugang - Struktur, Habitus und Praxis .................................. 23

2.3.2 Einverleibung der Gesellschaftsordnung durch soziale Exposition ............................. 28

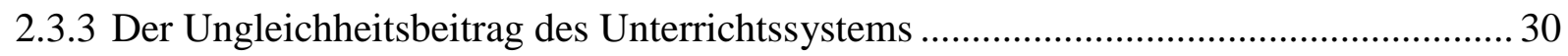

2.4 Lebensverlaufsperspektiven auf Gesundheit und Bildung ......................................... 33

2.4.1 Lebensverläufe als endogene Kausalzusammenhänge .............................................. 34

2.4.2 Modell kritischer Perioden: Schwangerschaft und frühe Kindheit............................... 36

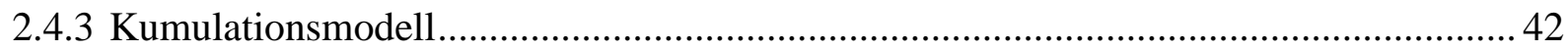

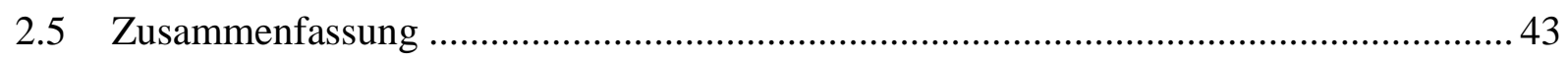

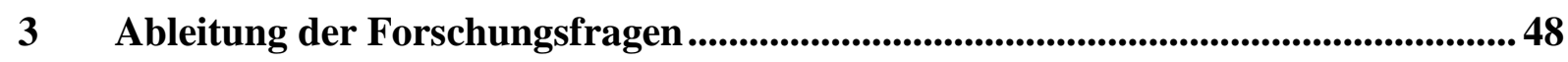

3.1 Herkunftsbedingte Ungleichheiten im Geburtsergebnis ............................................ 48

3.2 Herkunftsbedingte Ungleichheiten im Vorsorgestatus Neugeborener ......................... 49

3.3 Herkunftsbedingte Ungleichheiten im Bildungserfolg ............................................ 51

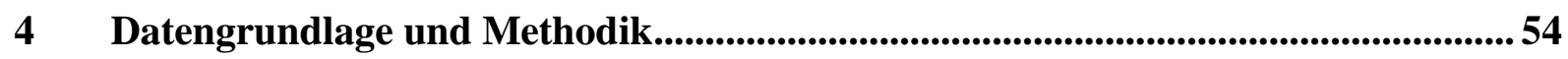

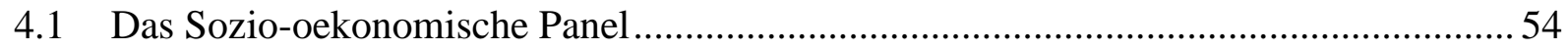




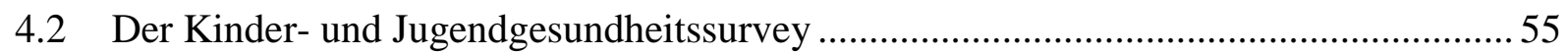

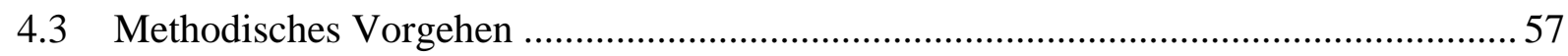

$5 \quad$ Gesundheitliche Ungleichheit zum Lebensbeginn .................................................5 58

5.1 Frühchen - ein medizinisches und soziales Problem..................................................... 58

5.2 Bourdieus Kapitaltheorie zur Erklärung gesundheitlicher Ungleichheit zum

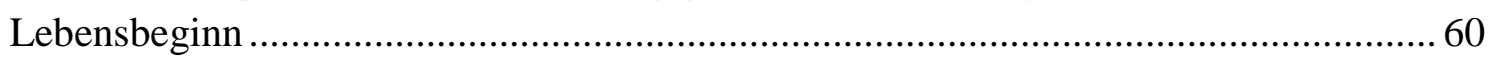

5.2.1 Das Kulturkapital als zentraler Frühgeburtsprädiktor ................................................ 61

5.2.2 Direkte und vermittelnde Effekte ökonomischen Kapitals ........................................... 62

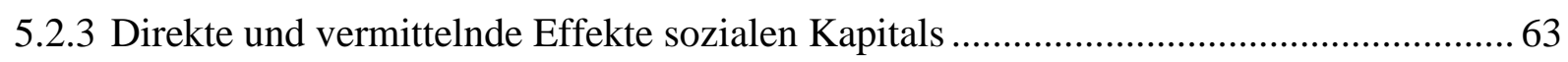

5.2.4 Habitus und Lebensstil als Bindeglieder zwischen Sozialstruktur und Gesundheit ....... 65

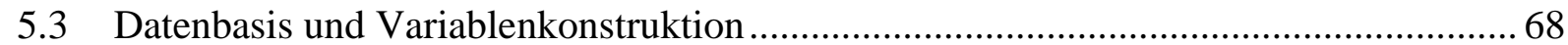

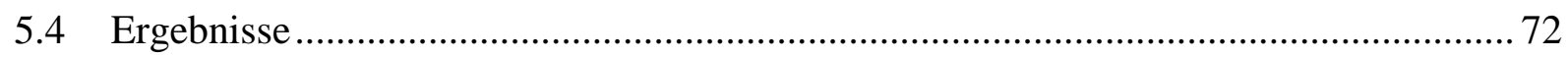

5.4.1 Die Modellvariablen nach mütterlichem Kulturkapital .............................................. 72

5.4.2 Mediatoren bildungsabhängiger Frühgeburtsrisiken …........................................... 75

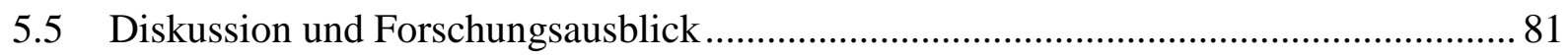

6 Gesundheitliche Ungleichheit im Vorsorgestatus Neugeborener ................................ 88

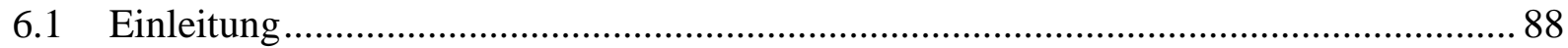

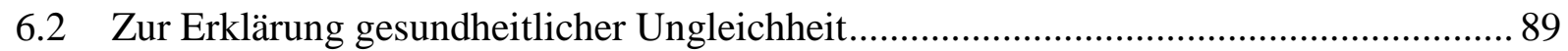

6.3 Das Sozialkapitalkonzept in der Gesundheitsforschung............................................ 91

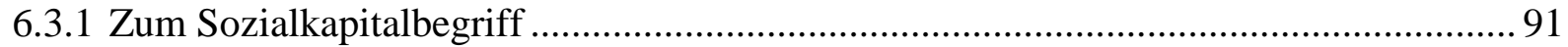

6.3.2 Die gesellschaftliche Verteilung sozialen Kapitals ................................................... 92

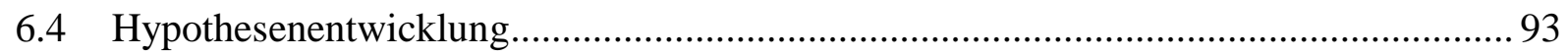

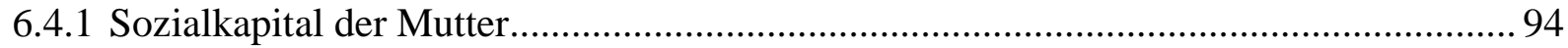

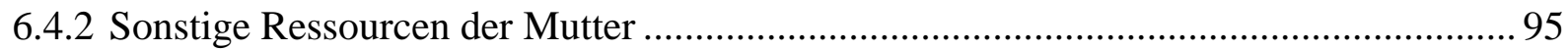

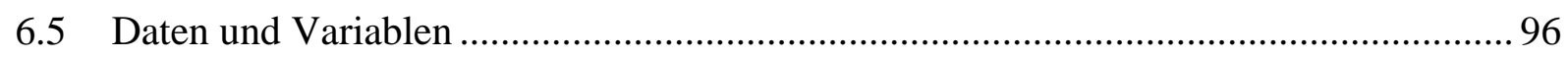

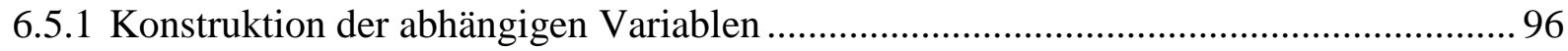

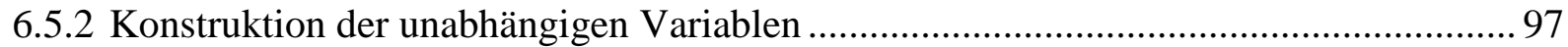

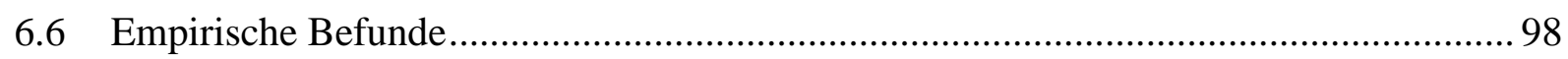

6.6.1 Sozialkapital: Ungleich verteilt nach Klassenlage und Bildung? ................................. 99

6.6.2 Die Teilnahme an einer altersgemäßen Vorsorgeuntersuchung für Kinder ................. 101

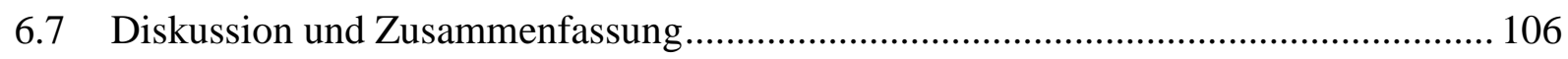




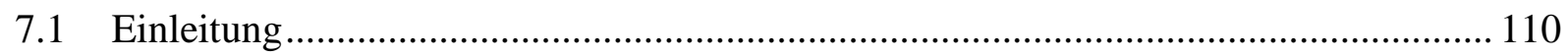

7.2 Zur Erklärung ethnischer Gesundheits- und Bildungsunterschiede ........................... 112

7.2.1 Zur Entstehung ethnischer Ungleichheit zum Lebensbeginn .................................... 113

7.2.2 Zur Entstehung ethnischer Ungleichheit im Kompetenz- und Bildungserwerb ........... 115

7.2.3 Schulische Konsequenzen lebensanfänglicher Entwicklungsnachteile ....................... 117

7.3 Datenbasis, Variablenkonstruktion und methodisches Vorgehen ................................ 118

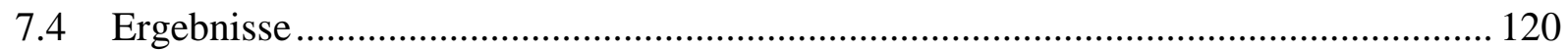

7.4.1 Deskriptive Statistiken zum Schulerfolg und den zentralen Modellvariablen ............. 120

7.4.2 Ethnische Disparitäten in der gesundheitlichen und schulischen Ausgangslage.......... 123

7.4.3 Ethnische Disparitäten in den Grundschulnoten und im Sekundarschulbesuch........... 127

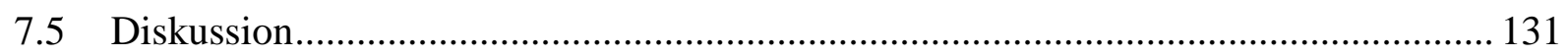

8 Diskussion und Zusammenfassung ..................................................................................... 137

8.1 Gesundheitliche Ungleichheit zum Lebensbeginn ................................................... 139

8.2 Ungleichheiten im Vorsorgestatus Neugeborener ...................................................... 143

8.3 Bildungsfolgen lebensanfänglicher Gesundheitsungleichheiten ............................... 146

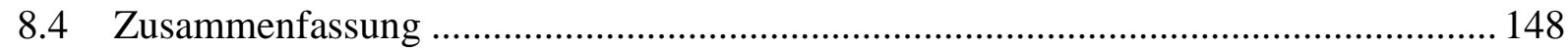

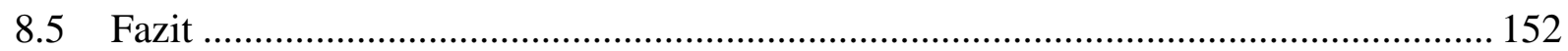

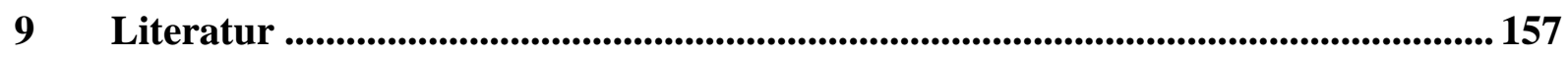




\section{Abbildungsverzeichnis}

Abbildung 2.1: $\quad$ Erklärungsmodell zur sozialen Statusvererbung......................................... 13

Abbildung 3.1: Erklärungsmodell zu herkunftsspezifischen Geburtsergebnissen................ 49

Abbildung 3.2: $\quad$ Erklärungsmodell zu herkunftsspezifischem Präventionsverhalten ............ 51

Abbildung 3.3: Erklärungsmodell zu herkunftsspezifischem Bildungserfolg ......................52

Abbildung 5.1: Die Kapitaltheorie zur Erklärung gesundheitlicher Ungleichheit................ 61

Abbildung 6.1: Sozialkapital der Mutter nach elterlicher Klassenlage (in Prozent)............. 99

Abbildung 6.2: Sonstige Ressourcen der Mutter nach elterlicher Klassenlage (in Prozent)

Abbildung 6.3: Beteiligung am Vorsorgeprogramm nach mütterlichem Bildungsabschluss

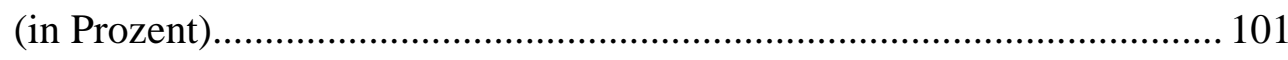

Abbildung 6.4: Beteiligung am Vorsorgeprogramm nach elterlicher Klassenlage (in

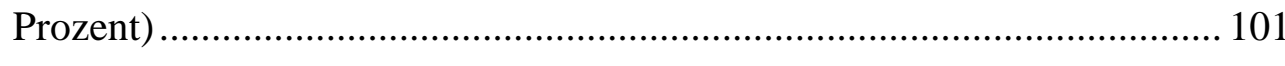

Abbildung 6.5: Beteiligung am Vorsorgeprogramm nach sozialem Kapital der Mutter (in

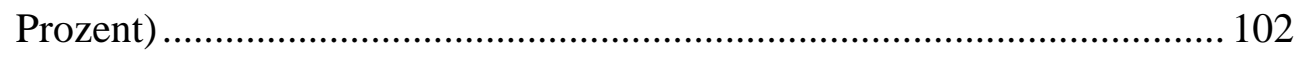

Abbildung 6.6: Beteiligung am Vorsorgeprogramm nach sonstigen Ressourcen der Mutter (in Prozent)............................................................................... 103

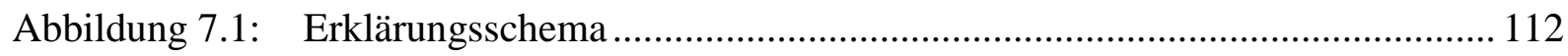




\section{Tabellenverzeichnis}

Tabelle 4.1: $\quad$ Vor- und Nachteile des Erhebungsprogramms und -designs von SOEP und

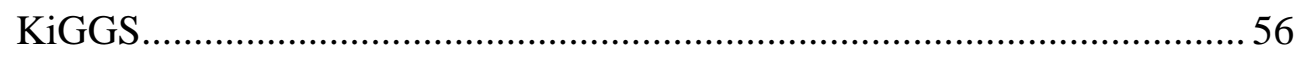

Tabelle 5.1: $\quad$ Verteilung der Frühgeburt nach mütterlichem Kulturkapital. ...................... 72

Tabelle 5.2: $\quad$ Verteilung des ökonomischen- und sozialen Kapitals und des

Gesundheitsverhaltens nach mütterlichem Kulturkapital

Tabelle 5.3: Logistische Regressionen zur Vorhersagewahrscheinlichkeit einer Frühgeburt (durchschnittlich marginale Effekte, multivariat) .................... 79

Tabelle A 5.1: Verteilung der Kontrollvariablen nach mütterlichem Kulturkapital............ 85

Tabelle A 5.2: $\quad$ Logistische Regressionen zur Vorhersagewahrscheinlichkeit einer

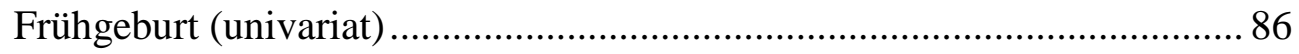

Tabelle A 5.3: $\quad$ Korrelationen zwischen einzelnen Variablen ............................................. 87

Tabelle 6.1: $\quad$ Sozialkapitaleinfluss auf die Teilnahme an altersgemäßer

Vorsorgeuntersuchung (logistische Regression, odds ratios und average marginal effects) .................................................................................. 103

Tabelle 6.2: $\quad$ Determinanten der Teilnahme an einer altersgemäßen Vorsorgeuntersuchung für Kinder (logistische Regression, odds ratios und average marginal effects) ................................................................ 105

Tabelle A 6.1: $\quad$ Überblick zur Komposition des Untersuchungssamples ........................... 109

Tabelle 7.1: $\quad$ Bildungsmerkmale nach ethnischer Herkunft (getrennt für Primar-/Sekundarstufe)

Tabelle 7.2: $\quad$ Säuglingsgesundheit, Schwangerschafts- und Familienmerkmale nach ethnischer Herkunft ${ }^{\text {a }}$.....

Tabelle 7.3: $\quad$ Logistische Regressionen zu Termingeburten und postnataler Probleme sowie lineare Regression des Geburtsgewichts auf die Modellvariablen .. 124

Tabelle 7.4: Zurückstellung vom Schulbesuch (logistische Regression, Gesamtstichprobe) ......................................................................... 126

Tabelle 7.5: $\quad$ Lineare Regression der Deutschnote (GrundschülerInnen) 129

Tabelle 7.6: $\quad$ Logistische Regression des Schulbesuchs (SekundarschülerInnen, inkl. Gesamtschülerschaft) ........................................................................ 130

Tabelle A 7.1: Lineare Regression der Mathematiknote (GrundschülerInnen)

Tabelle A 7.2: $\quad$ Logistische Regression des Schulbesuchs (SekundarschülerInnen, ohne Gesamtschülerschaft) ...................................................................... 136

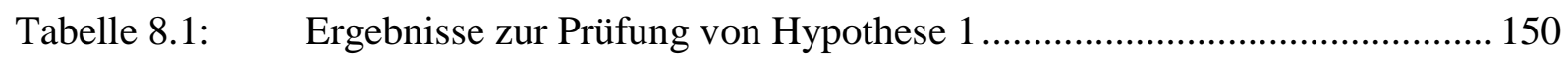

Tabelle 8.2: $\quad$ Ergebnisse zur Prüfung von Hypothese 2 ............................................... 151 


\section{Einleitung}

„Da die schulischen Leistungen von Arbeiterkindern und die darauf basierenden Bewertungen durch Lehrkräfte mit der sozialen Herkunft der Schulkinder zusammenhängen, besteht ihre Chancenungleichheit im Schulsystem im bildlichen Sinne darin, dass die Startchancen beim Hundertmeterlauf insofern ungleich nach sozialer Herkunft verteilt sind, als dass die Arbeiterkinder mit zu groß geratenen Schuhen ohne Schnürsenkel an der Startlinie stehen, während die Kinder aus höheren Sozialschichten mit bester Ausstattung einen nicht einholbaren Vorsprung von über 50 Meter haben, bevor überhaupt der Startschuss gefallen ist.“ (Becker und Lauterbach 2008: 134f)

„Bereits während der Schwangerschaft werden wichtige Voraussetzungen für die späteren Entwicklungschancen des Kindes im Rahmen primärer Sozialisation geschaffen (...) Verzögerte oder gestörte Reifung während der Schwangerschaft können Umfang und Tempo von Lernprozessen nachhaltig beeinflussen“ (Siegrist 2005: 91, Herv. i.O.)

\subsection{Problemstellung und Forschungsfragen}

Soziale Ungleichheiten in Gesundheit und Bildung erweisen sich als äußerst langlebige Phänomene und stellen westliche Gesellschaften vor zentrale politische Herausforderungen.

Im Bereich Gesundheit stellt die Zugehörigkeit zu einer niedrigen Sozialschicht die bedeutsamste Einflussgröße auf das Sterbe- und Krankheitsgeschehen dar, im Erwachsenen- wie im Jugend-, Kindes- und Säuglingsalter (Williams 1998). Empirisch zeigt sich: Je höher die auf der sozialen Leiter erklommene Stufe, desto besser die Gesundheit (Mielck 2005; Power und Kuh 2008). An diesem Tatbestand vermochten medizinisch-technologische Fortschritte oder der Ausbau des Gesundheitssystems nur wenig auszurichten (Siegrist und Marmot 2008: 16).

In ähnlicher Weise zeigen sich vertikale Bildungsungleichheiten als relativ änderungsresistent. Während sich horizontale Ungleichheiten, wie die nach Geschlecht ungleiche Bildungsbeteiligung, über die Zeit wandelten und zu Ungunsten von Jungen umkehrten oder aber neue Dimensionen hinzukamen, wie die ethnische Herkunft, ,ragen schichtspezifische Unterschiede weiterhin wie ein Fels in die Brandungswellen der Bildungsexpansion hinein“" (Geißler 1994: 111). Der massive Ausbau des deutschen Bildungssystems, insbesondere der weiterführenden Schulen, führte im 20. Jahrhundert zwar zu mehr Bildungschancen für alle Bevölkerungsgruppen, an den sozialen Ungleichheitsmustern in der Bildungsbeteiligung, im Kompetenz- und Leistungsstand hat sich aber nur wenig geändert (Geißler 1994; Becker und Lauterbach 2008). Über Erfolg oder Misserfolg im Bildungssystem entscheidet auch am Anfang des 21. Jahrhunderts noch immer die soziale und ethnische Herkunft, beispielsweise in Bezug auf die Chancen, regulär und nicht durch Rückstellung ein Jahr verspätet eingeschult zu werden (Kratzmann und Schneider 2009; Tuppat und Becker 2014), in der Primarstufe gute Noten zu erzielen, in der Sekundarstufe eine weiterführende Schule zu besuchen (Kristen 2002) und diese erfolgreich mit einem Bildungszertifikat abzuschließen.

Angesichts der Beständigkeit sozialer und ethnischer Strukturphänomene schaut die Bildungsforschung wenig optimistisch in die Zukunft: Solange sich Kinder beim Schuleintritt im Entwicklungsstand nach familiären Merkmalen unterscheiden, bleibt Chancengleichheit im 
Bildungswesen, so Rolf Becker und Wolfgang Lauterbach (2008: 36), wahrscheinlich eine „Utopie“, zumal Grundschulen individuelle entwicklungs- und kompetenzbezogene Nachteile in den Lernvoraussetzungen nur partiell kompensieren (Diefenbach 2011). Weil sich geringe Startnachteile im Bildungsverlauf verfestigen können (Becker und Biedinger 2006: 680), erscheint es notwendig, mehr Wissen über die biographisch frühe Genese herkunftsbezogener Ungleichheiten in Erfahrung zu bringen. Diesbezüglich finden in der Ungleichheitsforschung zwei Aspekte bislang noch zu wenig Beachtung, nämlich auf der einen Seite, dass Bildung bereits mit der Geburt beginnt (Schäfer 2003) und, auf der anderen Seite, dass soziale Unterschiede in der Lernausgangslage von Grundschülern und Grundschülerinnen auch über auf sozialem Wege erzeugte entwicklungsbiologische Risiken vermittelt sein können, wie etwa infolge einer ,zu frühen Geburt“(Jäkel et al. 2012: 149).

Wie Evidenzen belegen, zeigen sich nach Bildung und Herkunft der Mutter bedeutsame Differenzen in der Schwangerschaftsdauer (Behrman und Butler 2007; Raum et al. 2001; Razum et al. 2011). So entbinden Frauen aus unteren Bildungsschichten, insbesondere aber Migrantinnen häufiger zu früh, d.h. vor Vollendung der 37. Schwangerschaftswoche (Robert Koch-Institut 2008; Becker und Stolberg 2013). Von diesen Ungleichheitsbefunden nimmt die deutsche Bildungsforschung bislang kaum Notiz; die vorgeburtliche Lebensphase wird in soziologischen Erklärungen herkunftsabhängiger Bildungsdisparitäten zumeist ausgeblendet womöglich vorschnell angesichts empirisch bedeutsamer Relationen zwischen Frühgeburtlichkeit, niedrigem Geburtsgewicht auf der einen und schulischen Erfolgsindikatoren auf der anderen Seite (Lynch 2011: 523).

Für eine möglichst tiefgehende Erklärung sozialer und ethnischer Bildungsdifferenzen sind also leistungsrelevante Frühbedingungen zu berücksichtigen - von Anbeginn der Schwangerschaft (Kristen 2008: 231). Bezugnehmend auf das im Eingangszitat enthaltene Sinnbild für ungleiche Bildungswettbewerbsbedingungen, stellt sich deshalb die Frage, ob Ungleichheiten bei Neugeborenen zu Nachteilen beim „Hundertmeterlauf“ führen: Besteht der Vorsprung in den Lernvoraussetzungen bei Grundschulkindern aus oberen Sozialschichten (oder aus der einheimischen Mehrheitsgesellschaft) auch aus dem Grund, dass sie unter günstigeren Bedingungen im Mutterleib heranreifen und mit mehr oder weniger schwer einholbaren Entwicklungsvorteilen ins Leben starten? Könnten die Schuhe, in denen Arbeiterkinder (oder auch Migrantenkinder) beim Bildungswettlauf an die Startlinie treten, womöglich deshalb zu groß sein, weil für sie der Startschuss ins Leben, gemessen an den im Mittel höheren Frühgeburtsraten, vergleichsweise früh fällt?

Inwiefern sich die in der Gesundheitsforschung prominente „fetal origin“-These, gemäß welcher Ausgangsnachteile im Entwicklungsstand, wie sie über ein niedriges Geburtsgewicht abgebildet werden, ${ }^{1}$ die Gesundheitschancen von Säuglingen schmälern (Barker et al. 2002),

\footnotetext{
${ }^{1}$ Ein Lebendgewicht weniger als 2500 Gramm weist auf ungünstige Bedingungen in der fötalen Entwicklungsperiode hin und erhöht die Säuglingsmortalität (Dragano und Siegrist 2009: 185; Steinhausen 2000: 101 ff.)
} 
auch im Forschungsbereich zu ungleichen Bildungschancen bewährt, ist für Deutschland erst ansatzweise untersucht (Jäkel 2012; Jäkel et al. 2013).

Im Allgemeinen sind die Ursachen für soziale Entwicklungsdifferenzen zum Lebensbeginn nicht abschließend ermittelt, wobei neben den Lebensbedingungen das Gesundheitshandeln einen bedeutsamen Einflussfaktor darstellt (Behrman und Butler 2007; Siegrist 2005: 190). Aber gerade in Bezug auf die Erklärung sozialer Unterschiede im elterlichen Gesundheitshandeln bestehen größere Blindstellen im Forschungsstand. Neben lebensanfänglichen Gesundheitsnachteilen nimmt die vorliegende Arbeit deshalb auch die Gründe hinter sozial strukturierten Präventionspraxen genauer unter die Lupe, und zwar am Beispiel der Inanspruchnahme von Früherkennungsuntersuchungen für Kinder. Im Zusammenhang mit Schulerfolg interessiert dabei, ob Lücken im Vorsorgestatus, welche, so die Befundlage, in unteren Bildungsschichten (und Migrantenfamilien) prägnanter ausfallen, nicht nur soziale Gesundheitsunterschiede begünstigen (Jungbauer-Gans und Kriwy 2004: 15; Mueller und HeinzelGutenbrunner 2001: 9), sondern auch zur Entstehung sozialer Bildungsunterschiede beitragen.

Ein potentieller Erklärungsbeitrag scheint also durchaus plausibel, da frühe Entwicklungsbeeinträchtigungen, wie sie bei Frühgeborenen zumeist aus organischer Unreife (das Gehirn, Seh- und Hörvermögen betreffend) herrühren, die individuelle Aneignung lernrelevanter Kompetenzen und somit die kognitive Verarbeitung schulischer Inhalte behindern. Vermittelt über niedrige Schulleistungen können schwangerschaftsbezogene Entwicklungsnachteile, bleiben sie unerkannt und unbehandelt, den Weg in untere Bildungssegmente (Sonder/Hauptschule) vorbahnen (Petrou et al. 2001).

Vor diesem Hintergrund gewinnt die sozialinvestive Rhetorik moderner Wohlfahrtsstaaten, um jedes Kind zu kämpfen (Thaiss et al. 2010) an Nachdruck. Öffentliche Gesundheitsstrategien zielen darauf ab, allen Kindern, insbesondere jenen aus stärker risikobehafteten Familien mit geringem Sozialstatus (darunter insbesondere Migrantenfamilien) über den Präventionsweg ein gesundes Aufwachsen zu ermöglichen (Ostner und Stolberg 2015; Bermaoui et al. 2012). Kindliche Entwicklungs-, Lern- und Bildungsverläufe stehen zunehmend unter epidemiologischer, präventiver und pädagogischer Beobachtung. Dabei hat sich in Surveystudien gezeigt, dass neben Kindern aus unteren Sozialschichten auch Kinder aus Migrantenfamilien präventiv im Mittel weniger gut erreicht werden - pränatal (etwa im Rahmen der Vorsorgeuntersuchungen für Schwangere, Razum et al. 2011) wie auch postnatal (Kamtsiuris et al. 2007; Stich et al. 2009; Kurz und Becker 2017).

Eine gesetzlich verankerte Präventions-, Kompensations-, elterliche Beratungs- und Kontrollfunktion erfüllt das hier in den Untersuchungsfokus gerückte Krankheitsfrüherkennungsprogramm für Kinder, welches mit den sogenannten U-Untersuchungen darauf abzielt, frühe Entwicklungsbeeinträchtigungen frühzeitig zu erkennen und möglichst noch vor Schulbeginn auszugleichen (Thaiss et al. 2010).

Auf welchen Mechanismen herkunftsbezogene Ungleichheiten unter Neugeborenen und ihrem Vorsorgestatus beruhen und inwiefern eine Betrachtung dieser frühen gesundheitsbezogenen Ungleichheiten als Erklärungsgrößen helfen, die Entstehung sozialer und ethnischer 
Bildungsungleichheiten besser zu verstehen, stellt gegenwärtig noch ein Forschungsdesiderat dar. Diesem widmet sich die vorliegende Dissertation theoretisch wie empirisch, wobei die Leitfragestellung wie folgt lautet: Werden Gesundheit und Bildung in die Wiege gelegt?

Mit für Deutschland repräsentativen Daten, die einerseits vom Sozioökonomischen Panel (Befragungsdaten) und andererseits vom Kinder- und Jugendgesundheitssurvey (Befragungsund medizinische Untersuchungsdaten) stammen, wird nachfolgend drei Teilfragestellungen nachgegangen:

(1) Bestehen nach der sozialen und ethnischen Herkunft gesundheitliche Unterschiede zwischen Neugeborenen und wie lassen sich diese erklären?

(2) Warum nehmen Eltern mit einem formal unterschiedlichen Bildungsgrad in einem unterschiedlichen Umfang an den U-Untersuchungen für Kinder teil?

(3) Lässt sich die Herkunftsabhängigkeit des Bildungserfolgs über lebensanfängliche Gesundheitsdifferenzen und soziale Unterschiede im Gesundheitshandeln erklären?

\subsection{Relevanz und Zielstellung}

Warum handelt es sich bei den aufgeworfenen Fragen um soziologisch untersuchungswürdige Probleme? Ihre Relevanz kann aus verschiedenen Blickwinkeln begründet werden. Zunächst einmal haben Gesundheit und Bildung für das Individuum (z.B. mit Blick auf Arbeits- und Heiratsmarktchancen, soziokulturelle Teilhabe, alltägliche Lebensbewältigung, Lebenserwartung) wie für die Gesellschaft (kollektive Leistungsfähigkeit, Wohlstand) einen hohen Wert (Becker 1993; Hurrelmann 2006; Mirowsky und Ross 2003; Siegrist und Marmot 2008). Dementsprechend wird beiden Formen humanen Vermögens auch auf institutioneller Ebene Bedeutung verliehen: Gesundheit (WHO 1986; Hurrelmann 1988; Borgetto und Kälble 2007: 43) und Bildung (Dahrendorf 1965; Motakef 2006: 9) sind Menschen- bzw. Bürgerrechte.

Vor diesem Hintergrund erscheint es besonders problematisch, dass Neugeborene, wie eingangs illustriert, mit ungleichen Entwicklungsvoraussetzungen ins Leben starten. Ein gesundheitlich schlechter Start ins Leben stellt dabei nicht nur betroffene Familien, sondern die Gesellschaft vor verschiedene Herausforderungen. Für die Gesellschaft markieren Gesundheitsdisparitäten zum Zeitpunkt der Geburt eine soziale Frage bzw. ein öffentliches Gesundheitsproblem, das in seiner quantitativen Dimension in modernen Gesellschaften zukünftig an Bedeutung gewinnt (Kirschner et al. 2009). So werden Prävalenzen für Frühgeburten und niedriges Geburtsgewicht weiter ansteigen: Morbiditätsbedingt infolge der Aufschiebung des Kinderwunsches in höhere Lebensalter, durch die Zunahme von Mehrlingsschwangerschaften, was mit dem Anstieg künstlicher Befruchtungen in Verbindung steht, sowie im Zuge des medizinisch-technologischen Fortschritt selbst, der das Überleben immer jüngerer schon in der 23. Schwangerschaftswoche geborener Kinder ermöglicht (Goldberg und Divitto 2002).

Letzteres birgt wiederum gesundheitsökonomische Implikationen. So werden Frühgeborene häufig intensivmedizinisch versorgt, was das Gemeinwesen finanziell stark belasten kann. Ein Beispiel: Auf Basis von Krankenkassendaten sowie einer Befragung von Müttern im Ba- 
byCare-Programm ergibt sich zwischen Früh- und Termingeburten eine geschätzte Kostendifferenz von mindestens 10.550 Euro pro Fall (Kirschner et al. 2009), wobei es sich, wie das Autorenteam betont, um eine konservative Berechnung handelt, die nur in den ersten zwei Lebenstagen anfallende Versorgungskosten einbezieht - nicht aber Ausgaben im Zusammenhang mit stationären Wiederaufnahmen, der aufwendigen Kompensation entwicklungsbezogener Ausgangsnachteile oder therapeutischen Begleitung der Eltern, welche in Sorge um die Gesundheit ihres Nachwuchses starken psychosozialen Belastungen ausgesetzt sind.

Darüber hinaus werfen Ungleichheiten unter Neugeborenen womöglich auch ein bildungspolitisches Problem auf, ließe sich die in der vorliegenden Arbeit aufgestellte Anahme empirisch erhärten, dass sich gesundheitsbezogene Startnachteile nicht im Vorfeld der Schule verflüchtigen, sondern in systematische schulische Erfolgsnachteile übersetzen. Vorstrukturiert ein Frühstart ins Leben den Kompetenz- und Bildungserwerb, kann das für die „Bildungsausschöpfung“" gesamtwirtschaftliche Folgen haben, weil Leistungsvermögen, Produktivität und Wettbewerbsfähigkeit moderner Volkswirtschaften bedeutsam vom Bildungs- und Gesundheitszustand der Bevölkerung abhängen (Krüger-Hemmer 2016: 98).

Neben der ökonomischen Relevanz der Thematik kann eine Intensivierung der Forschung $\mathrm{zu}$ gesundheitlichen Ungleichheiten zum Lebensbeginn, einschließlich potentiell negativer Bildungskonsequenzen, darüber motiviert werden, dass in demokratisch verfassten Gesellschaften soziale Ungleichheitsphänomene das Prinzip sozialer Chancengleichheit verletzen (zu Bildung, Solga 2005).

Die Arbeit verortet sich in der soziologischen Ungleichheitsforschung, für die die Frage nach der Entstehung von sozialer Ungleichheit unter den Menschen zentral ist (Berger 2004). Indem den Gründen und Bildungsfolgen gesundheitlicher Ungleichheiten nachgegangen wird, besetzt die Arbeit die Schnittstelle zwischen der empirischen Bildungs- und Gesundheitsforschung. Beiden Forschungsbereichen ist mit Blick auf die Prozesse der sozialen Produktion und intergenerationalen Reproduktion von ungleichen Bildungs- und Gesundheitschancen sowohl eine quantitativ-empirische als auch ressourcen- und sozialisationstheoretische Ausrichtung gemein (für Gesundheit, Jungbauer-Gans 2006; für Bildung, Becker 2009). Trotz der offensichtlichen theoretisch-konzeptionellen Nähe stehen beide Bindestrichsoziologien derzeit jedoch relativ unverbunden nebeneinander.

Zur Beantwortung der drei aufgeworfenen Forschungsfragen gilt es demnach, Gesundheit und Bildung aufeinander zu beziehen. Hierfür wird das Augenmerk einerseits verstärkt auf Bourdieu's multidisziplinär anwendbare Kapital- und Habitustheorie gelegt. In der Gesundheitssoziologie wird Bourdieus Theorie den neuen (Abel 2008), in der Bildungssoziologie den etablierten Erklärungsansätzen zugeordnet (Becker 2009). Andererseits wird theoretisch auf die sozialepidemiologische Lebenslaufforschung abgestellt. Diese eignet sich für die Integration kapitaltheoretischer und entwicklungsbiologischer Faktoren, so dass ein Brückenschlag zu Bourdieus körpersoziologischer Einverleibungsthese hergestellt werden kann.

In diesem Kontext kommt der schwangerschaftsbezogenen Exposition zu sozioökonomischen und kulturell-verhaltensbezogenen Entwicklungsrisiken eine wichtige Rolle zu. Letzte- 
re wird soziologisch über herkunftsabhängige Denk-, Wahrnehmungs- und Handlungsorientierungen mit der Habitustheorie spezifiziert. Auch diesbezüglich trägt die Arbeit zum überschaubaren Forschungsstand bei; denn in der Forschungsliteratur bleiben Aussagen zur Ungleichheitswirksamkeit habitueller Dispositionen zumeist hypothetisch (für Gesundheit, Sperlich und Mielck 2003). Eine der für die Untersuchung verwendeten Datenquellen (Sozioökonomisches Panel) stellt Habitus-Informationen bereit und bietet die Möglichkeit, Bourdieus ungleichheitstheoretische Struktur-Habitus-Praxis-Formel empirisch auf Relevanz zu prüfen.

\subsection{Aufbau der Arbeit}

Im direkten Anschluss an die Einleitung führt Kapitel 2 in den konzeptionellen und theoretischen Rahmen ein. Neben frühen und neuen ungleichheitstheoretischen Bezügen werden ausgewählte Befunde berichtet, die der Untermauerung der theoretischen Argumentation dienen.

Der Einstieg in die theoretische Betrachtung erfolgt über die Statuserwerbsforschung (Abschnitt 2.1.1), die der ungleichheitssoziologischen Bildungsforschung in den 60er und 70er Jahren des 20. Jahrhunderts den Weg ebnete (Kristen 1999). Zusammen mit der Humankapitaltheorie (Abschnitt 2.1.2) und der Theorie sozialer Produktionsfunktionen (Abschnitt 2.1.3) bietet dieses Forschungsfeld einen wichtigen konzeptionellen Anker für die Problemstellung.

Über welche Prozesse und Mechanismen sozioökonomische Verteilungsungleichheiten und kulturell-verhaltensbezogene Bedingungen die Lebenschancen von Kindern prägen, wird sodann mithilfe zweier soziologischer Klassiker spezifiziert: Auf der einen Seite mit Boudon's Konzept primärer und sekundärer sozialer Herkunftseffekte (Abschnitt 2.2), das hauptsächlich auf Bildung (2.2.1) grundsätzlich aber auch auf Gesundheit angewandt werden kann (2.2.2) und, auf der anderen Seite, mithilfe Bourdieu's Kapital- und Habitustheorie (Abschnitt 2.3).

Beide theoretischen Konzepte sind in der Bildungsforschung populär geworden und beinhalten unterschiedlich differenzierte Begründungen für die Entstehung von Chancenungleichheiten. So entwirft Boudon ein rationaltheoretisches Konzept, das sich auf sozial differentielles Bildungsverhalten in Kontexten freier Schulwahl anwenden lässt (Stocké 2012), aber nur bedingt zur Erklärung sozialer Ungleichheiten im Schulerfolg eignet, da erfolgsrelevante Sozialisationspraxen nicht ausführlich behandelt und Institutioneneffekte ausgeblendet werden. Zur Erklärung von leistungs-, kompetenz- und gesundheitsbezogenen Disparitäten eignet sich Boudon's Ansatz daher nur begrenzt. Aus Gründen der Vollständigkeit - Boudon ist aus der Bildungsforschung nicht wegzudenken - und weil im dritten empirischen Teil (Kapitel 7) der Einfluss schwangerschaftsbezogener Faktoren nicht nur auf die Leistungen in der Grundschule, sondern auch auf die Bildungsbeteiligung getestet wird, soll und kann hier auf Boudon und das Konzept primärer und sekundärer Herkunftseffekte nicht verzichtet werden.

Unverzichtbar für die ressourcentheoretische Einbettung der Forschungsfragen ist die Gesellschaftstheorie Pierre Bourdieus. In dieser thematisiert Bourdieu ausführlicher als Boudon, auf welche Art und Weise familiäre und institutionelle Bedingungen zu Erfolg oder Misserfolg im Bildungssystem beitragen. Darüber hinaus erweist sich Bourdieus Habitustheorie als 
fruchtbar zur Erklärung gesundheitlicher Unterschiede (Abel 2009), wie sie hier in Gestalt gruppenspezifischer Gesundheitschancen zum Lebensbeginn im Untersuchungsfokus stehen.

Für die theoretische Rahmung der Untersuchungsfragen liefern also beide soziologischen Klassiker wichtige Ungleichheitsperspektiven, wobei jedoch Bourdieu mit seinen Schriften zur Einverleibung der Sozialwelt, in welche Ideen und Befunde aus wichtigen soziologischen Nachbardisziplinen, wie der Anthropologie, Erziehung und Psychologie einfließen, den theoretisch differenzierteren und kompletteren, Makro-, Meso- wie Mikro-Ebene berücksichtigenden Zugang eröffnet, um der Relation zwischen lebensanfänglicher Gesundheit und späterem Bildungserfolg auf den Grund zu gehen.

Da herkunftsspezifische Gesundheits- und Bildungsdisparitäten im Laufe vor- wie nachgeburtlicher Lebensphasen erzeugt werden - wobei das Zeitfenster der Schwangerschaft von der herkömmlichen Ungleichheitssoziologie bislang nur einen Spalt breit geöffnet wurde - ist es sinnvoll und notwendig, das in der Bildungs- wie Gesundheitsforschung angewandte Lebenslaufparadigma in den Theorierahmen zu integrieren (Abschnitt 2.4). Das Augenmerk liegt dabei auf dem sozialepidemiologischen Lebenslaufansatz und zwei theoretischen Ansätzen, mit deren Hilfe der Einfluss schwangerschaftsbezogener Gesundheits- und Bildungsdeterminanten modelliert werden kann. Von unmittelbarem Interesse hierbei sind das Modell kritischer Perioden (2.4.2), das sich auf die Zeit vor der Geburt sowie auf die frühe Kindheit bezieht, sowie das Modell der Akkumulation gesundheitlicher und bildungsbezogener Vor- und Nachteile im Lebenslauf (2.4.3). Beide Modelle sind eher als sich ergänzende als miteinander konkurrierende Erklärungskonzepte zu verstehen. Den Kapitelabschluss bildet ein die theoretischen Überlegungen zusammenfassender Abschnitt (2.5).

Aufbauend auf den Theorieteil erfolgt in Kapitel 3 die Ableitung der Forschungsfragen. Zur Veranschaulichung der Grundannahmen werden zu jedem Forschungsproblem modellhaft Erklärungsskizzen angefertigt. Einen Überblick zu den verwendeten Datenbasen und das methodische Vorgehen gibt Kapitel 4. Entwicklung und Überprüfung der zentralen Hypothesen erfolgen in den drei empirischen Hauptkapiteln, beginnend mit Kapitel 5 zu gesundheitlichen Ungleichheiten zum Lebensbeginn, gefolgt von Kapitel 6, das sich mit schichtspezifischen Unterschieden im Vorsorgestatus Neugeborener befasst, bevor Kapitel 7 die Bildungsfolgen lebensanfänglicher Startnachteile zum Untersuchungsgegenstand erhebt. In Kapitel 8 werden schließlich zentrale Befunde der Arbeit zusammengefasst und diskutiert. 


\section{Theoretische Bezüge und Befunde zu sozialen Chancenungleichheiten}

Der Entstehung herkunftsspezifischer Gesundheits- und Bildungsdisparitäten liegt nach jetzigem Wissensstand ein komplexes Zusammenspiel multipler ökonomischer, sozialer, kultureller, psychologischer, institutioneller und - in der Ungleichheitssoziologie in der Regel nur am Rande beleuchtet - entwicklungsbiologischer Faktoren zugrunde. Um nachzuvollziehen, wie Unterschiede im Humanvermögen der jüngsten Gesellschaftsmitglieder strukturiert werden, bedarf es demzufolge einer interdisziplinären Erklärungsperspektive. Diese wird in diesem Kapitel entlang in der empirischen Bildungs- und Gesundheitsforschung etablierter Ressourcentheorien und in der Sozialepidemiologie angewandter lebenslauftheoretischer Ansätze zur Modellierung der Herkunftsunterschiede in den Lebenschancen von Kindern entwickelt. ${ }^{2}$

Die konzeptionellen und theoretischen Überlegungen werden angeleitet durch die Arbeitshypothese, dass herkunftsspezifische Ungleichheiten beim Bildungserwerb neben differentiellen Sozialisationserfahrungen auch von lebensanfänglichen Ungleichheiten im Entwicklungsstand herrühren. Da schulische Erfolgschancen neben dem familiären Hintergrund auch maßgeblich von den Institutionen des Bildungssystems abhängen, wird im Theoriekapitel auch die potentiell ungleichheitsstiftende Rolle des Unterrichtssystems problematisiert.

\subsection{Konzeptionelle Verortung}

\subsubsection{Statuserwerbsforschung - Ungleichheit als mehrstufiger Prozess}

Für die Erklärung der sozialen Produktion und Reproduktion sozialer Ungleichheit geht die Statuserwerbsforschung von einem mehrstufigen Prozess aus, d.h. der Sozialstatus wird über mehrere Etappen im Lebenslauf individuell erworben (Boudon 1974: 21). Auf der ersten Prozessstufe durchlaufen Kinder aus Familien mit verschiedenem sozialem Hintergrund mit unterschiedlichem Erfolg das Bildungssystem, das sie mit mehr oder weniger wertvollen Bildungstiteln verlassen. Dieser über das Bildungssystem laufende Prozess, bei dem Kinder aus sozial weniger privilegierten Familien vergleichsweise selten als Gewinner hervorgehen, wird als ,inequality of educational opportunity“ (IEO) bezeichnet (Esser 1999: 317).

Auf der zweiten Stufe der intergenerationalen Statusvererbung übersetzen sich unterschiedlich angesehene (Aus-)Bildungsabschlüsse in differentielle arbeitsmarktbezogene Verwertungschancen: Im Beschäftigungssystem bringen Hochschulabschlüsse Jobvorteile, die wiederum den Boden für eine dem Bildungsgrad entsprechend hohe soziale Positionierung bereiten. Dieser zweite Ungleichheitsprozess wird als ,inequality of social opportunity“ (ISO) bezeichnet. Beide Prozesse zusammen, also IEO und ISO, stellen ein „doppeltes, hintereinander an-

\footnotetext{
${ }^{2}$ Bei Lebenschancen handelt es sich um ein Konzept zur Analyse gesellschaftlich ungleicher Entfaltungsmöglichkeiten (Dahrendorf 1979: 92); hier bezieht sich der Begriff auf herkunftsspezifische Chancen zur Verwirklichung gesellschaftlich anerkannter Wünsche oder Ziele in den Bereichen Gesundheit und Bildung.
} 
geordnetes Problem der soziologischen Erklärung“ dar (Esser 1999: 318), wobei ISO im Gegensatz zu IEO relativ gut verstanden wird.

Verdienst der Statuserwerbsforschung ist der mithilfe regressionsanalytischer Methoden (Pfadanalysen) erbrachte Nachweis (Blau und Duncan 1967; Sewell et al. 1969; für Deutschland, Müller 1975), dass die Sozialstruktur, in Gestalt des elterlichen (väterlichen) Bildungsgrads, kausal auf individuelle Lebenschancen der Kindergeneration (Söhne) wirkt. Da Bildung nicht als Erklärungsproblem im Untersuchungsfokus stand, wurden die Theoriemodelle sehr sparsam angelegt (Kristen 1999: 13). Folglich wurden Determinanten sozialer Bildungsungleichheiten nur ansatzweise ermittelt. Selbiges gilt teilweise für die aktuelle Bildungsforschung, welche soziale und ethnische Kompetenzunterschiede im Grund- und Vorschulalter oftmals nur partiell aufklärt (Kristen 2008). Liefert die Berücksichtigung vorschulischer und vorgeburtlicher Bildungsfaktoren die fehlenden Puzzlestücke zur Vervollständigung des sozialen Ungleichheitsbildes? Hierbei handelt es sich letztlich um eine empirisch zu klärende Frage, die in Kapitel 7 beantwortet wird. Aus theoretischer Sicht besteht nach gegenwärtiger Forschungslage nun berechtigterweise Grund anzunehmen, dass vorgeburtliche Bedingungen für den Bildungs- und damit für den sozialen Statuserwerb bedeutsam sind (Jackson 2013). Vor diesem Hintergrund müsste das herkömmlicherweise zwei Stufen umfassende soziale Reproduktionsmodell um einen entwicklungsbiologischen Übergang erweitert werden, und zwar der Transition von intrauteriner Umwelt (Mutterleib) in die extrauterine Sozialwelt: Der Geburt.

Ausgangspunkt für die soziale (Re-)Produktion sozialer (Bildungs-)Ungleichheiten sind demnach Prozesse, welche zu sozialer Ungleichheit in der entwicklungsbezogenen Ausgangslage Neugeborener führen. So ist denkbar, dass nachteilige vorgeburtliche Entwicklungsbedingungen individuelle Entwicklungsnachteile zum Zeitpunkt der Geburt (z.B. in Form niedrigen Geburtsgewichts) begünstigen, welche je nach Schweregrad familiär oder außerfamiliär unternommene Bildungsanstrengungen unterwandern können; mit dem Ergebnis einer sozial differentiellen Gefährdung des Bildungserfolgs zu Ungunsten unterprivilegierter Gruppen.

Konzeptionell kann soziale Ungleichheit zum Lebensbeginn als IEO und ISO vorgelagertes Erklärungsproblem modelliert und in Anlehnung an Boudons Terminologie als inequality of health opportunity $(\mathrm{IHO})$ bezeichnet werden. Im Fokus dieses dritten Prozessabschnitts steht die in der Regel 40 Wochen dauernde Schwangerschaft. Für das Ungeborene wie für die werdende Mutter stellt diese Zeit eine hochvulnerable kritische Lebensphase dar (Siegrist 2005), die entwicklungswidrigen sozialen Umweltbedingungen eine leichte Angriffsfläche bietet, mit womöglich negativen Folgen für die Gesundheits- und Bildungschancen Neugeborener.

In diesem Problemkontext umfasst das in Abbildung 2.1 vorgeschlagene soziale Reproduktionsmodell (kurz: iHES-Modell: Inequality of Health, Educational and Social Opportunity) statt der bisher zwei nunmehr drei Stufen. Soziale Unterschiede im Gesundheitsstatus Neugeborener werden demnach als soziologisches Teilproblem der intergenerationalen Statusvererbung konzeptualisiert, ein Problem, das für sich genommen aber auch in Bezug auf potentielle Beeinträchtigungen des Bildungserwerbs eine hohe Untersuchungsrelevanz besitzt. 
Im Folgenden stellt sich somit die soziologische Aufgabe, die in der Abbildung 2.1 dargestellten Relationen zwischen den Sozialstrukturgliedern Herkunft - Gesundheit - Bildung theoretisch zu spezifizieren. Hierfür wird der (ungeborene) Nachwuchs als Adressat gesundheitsbezogener und pädagogischer Maßnahmen auf der einen Seite und die Eltern als primäre und Schulen als sekundäre Vermittlungsinstanzen auf der anderen Seite betrachtet.

Abbildung 2.1: Erklärungsmodell zur sozialen Statusvererbung

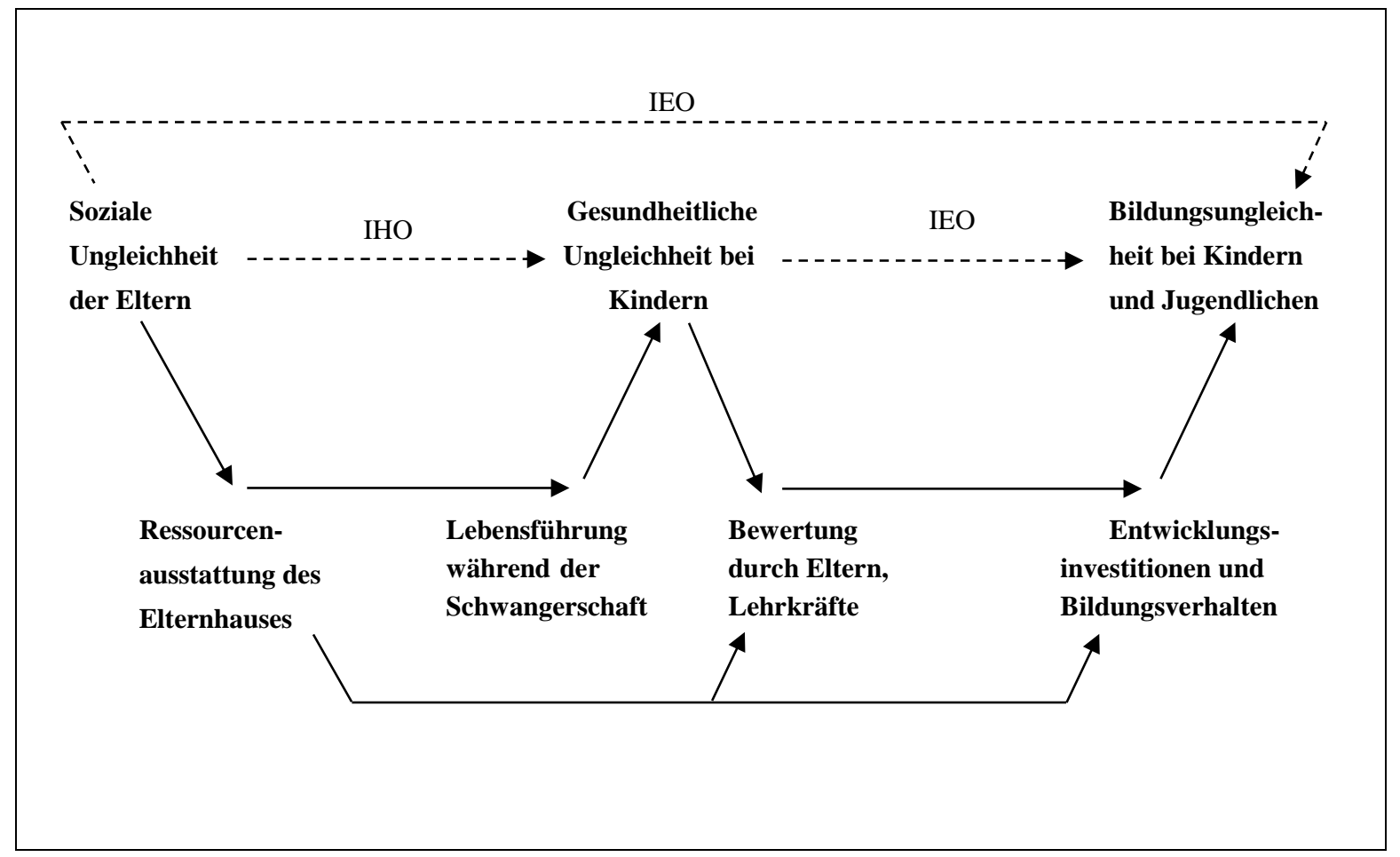

Anmerkung: Modifizierte Darstellung nach Esser (2000: 319); IHO (inequality of health opportunity), IEO (inequality of educational opportunity), nicht dargestellt ist der Prozess der Arbeitsmarktplatzierung (inequality of social opportunity).

\subsubsection{Humankapitaltheorie - Bildung und Gesundheit als Investition}

Mit der Humankapitaltheorie steht ein ökonomischer Zugang zu den Forschungsproblemen zur Verfügung. Bildung und Gesundheit können als Formen humanen Vermögens verstanden werden (Schultz 1961; G.S. Becker 1993). Beide Vermögensformen sind unauflöslich mit dem Körper des Individuums verbunden. In die Entwicklung, Gesundheit und Bildung von Kindern zu investieren lohnt sich nach dem Humankapitalansatz, weil eine Reihe monetärer und nicht-monetärer Erträge im Schul- und Erwachsenenalter an den produktiven Einsatz von Bildung und Gesundheit geknüpft. Bildung und Gesundheit werden primär mithilfe familiärer Entwicklungsinvestitionen von Kindesbeinen an individuell erworben. Der Idee, dass der Erwerb von Bildungs- und Gesundheitsvermögen über die Mitarbeit der Eltern läuft, kommt in anderen Theorien, wie etwa in sozialisationstheoretischen Ansätzen, auch ein zentraler Stellenwert zu, wird dort aber, im Gegensatz zum Humankapitalansatz, nicht ausschließlich ökonomisch begründet, sondern auch über kulturell tradierte bildungs- und gesundheitsbezogene Werte, Normen und Verhaltensweisen (Weber 1994; Jungbauer-Gans 2006). 
Indem der Humankapitalansatz soziokulturelle Bedingungen für kognitive Stimulation und ein gesundes Aufwachsen ausblendet, weist dieser einen vergleichsweise geringen Mehrwert für die Erklärung ungleichheitstheoretischer Fragen und damit eine größere Problemreihe auf: Da gesundheits- und bildungsbezogene Investitionsneigungen und Akkumulationsbedingungen vom sozialen und kulturellen Herkunftskontext abgekoppelt werden, kann die Humankapitaltheorie nicht überzeugend erklären, warum Menschen aus sozial weniger begünstigten Gruppen im Mittel eine geringere Investitionsneigung aufweisen und sich entgegen der ökonomischen Rationalitätsprämisse verhalten (Bourdieu 1983). Eine allgemeine Kosten-NutzenArgumentation ohne Einbezug feldspezifischer soziokultureller, psychosozialer und institutioneller Handlungsspielräume kann demnach sozial differentielle Gesundheits- und Bildungsaktivitäten und daraus resultierende Chancenungleichheiten nicht befriedigend erklären.

Es empfiehlt sich daher das zu bildende Theoriegerüst auf ein stärker soziologisch bewährtes, sozial differentielle Handlungsrestriktionen in Rechnung stellendes Fundament zu stellen. Hierfür bietet die Theorie sozialer Produktionsfaktoren (Lindenberg 1989) einen übergeordneten handlungstheoretischen Anknüpfungspunkt. Ihre zentrale Annahme lautet, dass Menschen gesundheits- und sozialstatusbezogene Grundbedürfnisse haben. Allerdings ist deren Befriedigung an günstige soziö̈konomische Bedingungen gebunden ist, welche in der Bevölkerung mehr oder weniger ungleich verteilt sind.

\subsubsection{Theorie sozialer Produktionsfaktoren - Bildung und Gesundheit als Bedürfnis}

Die Theorie sozialer Produktionsfaktoren (Lindenberg 2001) postuliert, dass alle Menschen unabhängig ihrer Position im sozialen Ungleichheitsgefüge ähnlich gelagerte bzw. universelle Ziele verfolgen, wie etwa einen hohen Sozialstatus zu erlangen. Analog gehört der Wunsch, „ein gesundes und langes Leben zu führen, (..) zu den menschlichen Bedürfnissen von hoher Priorität" (Weber 1994: 195). Die Grundannahme, dass Menschen neben sozioökonomischen Zielen nach physischem Wohlbefinden streben (Lindenberg 2001; Ormel et al. 1997), lässt sich auch auf die Gesundheit und Bildung von Kindern übertragen. Mit der Universalannahme kann argumentiert werden, dass sich alle Eltern nur das Beste für den Nachwuchs wünschen - in schulischer wie gesundheitlicher Hinsicht. Im Volksmund heißt es daher in Erwartung der Ankunft des (ungeborenen) Nachwuchses: Hauptsache gesund! Analog lässt sich für Bildung festhalten: Eltern wünschen sich im Allgemeinen, das heißt schichtübergreifend, eine möglichst gute (Aus-)Bildung für die eigenen Kinder (Geißler 1994: 142).

$\mathrm{Ob}$ die in der gesamten Bevölkerung vorhandenen Grundbedürfnisse verwirklicht werden, hängt entscheidend von Verfügbarkeit und Einsatz sogenannter instrumenteller, dem jeweiligen Universalziel hierarchisch untergeordneter Zwischenziele bzw. Zwischengüter ab. Zu letzteren zählt eine privilegierte Ausstattung mit ökonomischen, kulturellen und sozialen Ressourcen. Die Sozialschichtzugehörigkeit steckt dabei den Rahmen dafür ab, welche instrumentellen Zwischenziele bzw. -güter zur Realisierung der Wünsche nach Gesundheit und Bildung ausgewählt und ,produktiv eingesetzt werden können“ (für Bildung, Dollmann 2010: 24; für Gesundheit, Ormel et al. 1997). Soziale Ressourcenungleichheiten in der Elterngenera- 
tion führen somit in der logischen Konsequenz zu gesundheitlichen und bildungsbezogen Ungleichheiten in der Kindergeneration.

Wie Chancenunterschiede in den Dimensionen Bildung und Gesundheit erzeugt werden, wird nachfolgend mit zwei in der Ungleichheitsforschung etablierten Ansätzen aufgezeigt. Den Anfang macht das im internationalen Forschungsraum vielzitierte Konzept primärer und sekundärer sozialer Herkunftseffekte nach Raymond Boudon (Abschnitt 2.2), das sich herkömmlicherweise auf soziale Ungleichheiten in der Bildungsbeteiligung (2.1.1) aber auch auf Gesundheit anwenden lässt (2.1.2). Im Anschluss wird auf Pierre Bourdieus Kapital- und Habitustheorie für eine tiefergehende Ungleichheitserklärung zurückgegriffen (Abschnitt 2.3).

\subsection{Boudon's mikrosoziologischer Erklärungsansatz}

Boudon's mikro-soziologischer Erklärungsansatz besitzt einen Klassikerstatus in der internationalen Bildungsforschung. ${ }^{3}$ Im nationalen wie internationalen Forschungsraum bildet das Konzept primärer und sekundärer sozialer - und in neueren Arbeiten auch ethnischer (u.a. Dollmann 2010) - Herkunftseffekte den theoretischen Rahmen für unzählige Studien zur Genese von sozialen Ungleichheiten an den zentralen Gelenkstellen des Bildungssystems, wie dem Übergang von der Primar- in die Sekundarstufe oder vom Sekundar- ins Hochschulsystem. In jüngster Zeit erfährt das Konzept eine Ausweitung auf vorlagerte Bildungspassagen, wie der Transition in die Grundschule, bei dem sich Einschülerinnen und Einschüler je nach sozialer und ethnischer Familienherkunft bedeutsam voneinander in ihrer physischen, psychosozialen und kognitiven Entwicklung unterscheiden (Tuppat und Becker 2014). Diese Befunde geben wiederum Anlass, den Anwendungsbereich des Konzepts auf frühkindliche und vorgeburtliche Bildungsdeterminanten auszuweiten, womit sich unmittelbare Bezüge zur Gesundheit Neugeborener ergeben. Bevor das Konzept allgemein auf Gesundheit angewandt wird, steht Boudon's klassischer Gegenstand im Fokus - Herkunft und Bildung.

\subsubsection{Herkunft und Bildung}

In der klassischen Sichtweise werden Bildungsungleichheiten neben genetisch bedingten Begabungsunterschieden vor allem über zwei soziale Mechanismen erzeugt, wobei jeweils die Familienposition im sozialen Stratifikationssystem eine wichtige Rolle spielt (Boudon 1974).

Auf der einen Seite bieten Familien während der Sozialisation je nach sozialer Zugehörigkeit unterschiedliche kulturelle Anregungs- und Lernmöglichkeiten, welche im Vorschulalter zu spezifischen Entwicklungs- und Kompetenzunterschieden zu Ungunsten von Kindern aus sozial weniger privilegierten Familien führen (Sozialisationseffekt bzw. primärer Effekt der sozialen Herkunft). Schul- und lernrelevante Kompetenzrückstände schlagen sich wiederum in schlechten Schulnoten nieder, welche in nach Leistung selektierenden Bildungssystemen, wie dem deutschen System, die Chance signifikant reduzieren, eine zur Hochschule führende

\footnotetext{
${ }^{3}$ Das Konzept primärer und sekundärer sozialer Stratifikationseffekte findet sich bereits bei den Forschern Girard und Bastide (1963) (vgl. Neugebauer 2010).
} 
Schule zu besuchen. Auf der anderen Seite treffen Eltern im Bildungssystem an institutionell festgelegten Verzweigungspunkten je nach ökonomischen, kulturellen und sozialen Kapitalbesitz eine mehr oder weniger ambitionierte Schulwahl (Entscheidungseffekt bzw. sekundärer sozialer Herkunftseffekt). Aus dem Wechselspiel beider Stratifikationseffekte resultiert das in allen westlichen Gesellschaften beobachtbare je nach Länderkontext mehr oder weniger stark akzentuierte Phänomen der schichtspezifischen Bildungsbeteiligung.

Welche mit dem sozialen Familienhintergrund verknüpften Prozesse und Barrieren liegen dieser Ungleichheitserscheinung nun genau zugrunde? Nach Boudon spielen hierfür soziale Unterschiede im Bildungsverhalten die entscheidende Rolle. Im Vergleich zu Grundschulkindern aus sozial weniger privilegierten Familien setzen Kinder aus Familien am oberen Ende der Sozialhierarchie ihren Bildungsweg auch gegen Widerstände, wie negative Lehrerurteile oder Empfehlungen, durchschnittliche Leistungen oder Leistungskrisen (Geißler 1994: 142), überproportional häufig in einer weiterführenden Sekundarschulform fort. Ein wichtiger Grund hierfür sind in Abhängigkeit der sozialen Herkunft variierende bildungsbezogene Orientierungen, Wertschätzungen und Verhaltensweisen. Um das Risiko eines sozialen Statusverlustes in der Generationenfolge zu minimieren, haben Familien aus höheren Schichten keine andere Wahl als den potentiell ertragreichsten Bildungsgang anzustreben, in Deutschland das Gymnasium. Dem in höheren Schichten ,stärkeren Druck zu hohen Ausbildungsabschlüssen [steht in unteren Sozialschichten nur] die vage Hoffnung auf sozialen Aufstieg“ gegenüber (Geißler 1994: 144), wobei der erfolgreiche Besuch einer mittleren oder unteren Schullaufbahn zumeist für den Statuserhalt in unteren Sozialschichten ausreicht.

Der erwartete Nutzen einer höheren Schulbildung ist in höheren Schichten somit wesentlich höher anzusetzen als in unteren Schichten. Zudem fällt die Distanz zwischen elterlichem Sozialstatus und höherem Schulabschluss für Kinder aus unteren Sozialschichten relativ groß aus. Für Arbeiterfamilien wiegen deshalb die Kosten für das Zurücklegen des relativ langen (gymnasialen) Bildungsweges ungleich schwerer. Gleichzeitig wird in diesen Familien, aufgrund geringer Unterstützungsressourcen und fehlender Erfahrung mit den tatsächlichen Anforderungen höherer Schullaufbahnen, das schulische Misserfolgsrisiko subjektiv höher eingeschätzt. Deshalb wird die Entscheidung für anspruchsvolle Schullaufbahnen mit abnehmender Sozialschicht stärker vom individuellen Leistungsvermögen diktiert (Boudon 1974).

Zur Bewältigung der vom Standpunkt der Arbeiterfamilien aus betrachtet relativ großen sozialen Distanz zu den gesellschaftlich angesehensten Bildungssegmenten, müssen diese im Vergleich zu Akademikerfamilien im Mittel mehr Ressourcen aufwenden, eine höhere Motivation aufweisen sowie größere Lernanstrengungen unternehmen, um sich an Bildungsübergängen für höhere Bildungswege zu entscheiden (Boudon 1974). Untere Schichten tendieren daher verstärkt zur Wahl eines weniger anspruchsvollen Schultyps, selbst bei vergleichbaren Leistungen in den übergangsrelevanten Fächern oder Sekundarschulempfehlungen. Empfehlungen der Grundschullehrkräfte für untere Schullaufbahnen widersetzen sich dabei höhere Schichten eher als niedrigere Schichten, was die sozialen Differenzen in den Übergangsraten auf höhere zur Universität führende Bildungswege zusätzlich vergrößert. 
Vor diesem Hintergrund haben Kinder aus sozial privilegierten Familien gegenüber jenen aus weniger privilegierten Familien einen mehrfachen Bildungsvorteil (Dollmann 2010: 18). Einerseits werden sie im Vorschulalter aufgrund der relativen sozialen Besserstellung besser von ihren Familien auf schulische Lern- und Leistungsanforderungen vorbereitet, was folglich relative Notenvorteile bringt und damit, insofern Noten die Hauptübergangsdeterminanten darstellen, auch bessere Chancen auf einen Übergang in das Gymnasium. Andererseits streben Eltern aus oberen Sozialschichten, um einen sozialen Statusabstieg zu vermeiden, verstärkt die anspruchsvollsten Schulen in der Sekundarstufe an, und zwar selbst dann, wenn ihre Sprösslinge nur (unter-)durchschnittliche Noten erzielen. Familien mit ausreichend ökonomischem Kapital können bei eventuell auftretenden schulischen Schwierigkeiten private Nachhilfe finanzieren, oder, da sie häufig selbst das Gymnasium durchlaufen sind und daher mit den Leistungsanforderungen vertraut sind, selbst Hilfestellung in schulischen Angelegenheiten geben, was subjektive mit der Entscheidungssituation verbundene Unsicherheiten (ob des zukünftigen Schulerfolgs) aufseiten der Eltern und aufseiten der Kinder reduziert.

Sekundäre Herkunftseffekte treten darüber hinaus auch in Form höheren elterlichen Engagements in der Schule in Erscheinung. Eltern aus oberen Bildungsschichten arbeiten vergleichsweise intensiv mit der Lehrerschaft zusammen, zeigen Präsenz bei Elternabenden und arbeiten aktiv in Gremien mit (Elternvertretung, Elternbeirat), was sich wiederum positiv auf die Schulerfolgschancen ihrer Kinder auswirken kann (Geißler 1994: 143). Vermutlich verhalten sich obere Sozialschichten vor dem Übergang in die Sekundarstufe strategisch vorausschauend, um zu vermeiden, dass der Nachwuchs am Ende der Grundschullaufbahn die Leistungshürde für das Gymnasium reißt. Die Erhöhung der Übergangschancen kann direkt erfolgen über die Kontaktaufnahme mit Klassenlehrerinnen und -Lehrern (Geißler 1994: 143) oder indirekt, über die Erhöhung des Leistungsdrucks auf die Kinder oder durch Bereitstellung zusätzlicher Unterstützung (Nachhilfe) zur kurzfristigen Steigerung des Leistungsvermögens. Umgekehrt kann angenommen werden, dass Schülerinnen und Schüler eine geringere Leistungsmotivation aufweisen, wenn sie davon ausgehen, die für den Übergang in das Gymnasium erforderliche Leistungshürde nicht zu meistern (Jackson 2013).

Wie diese Beispiele illustrieren, lassen sich primäre und sekundäre soziale Herkunftseffekte theoretisch nicht trennscharf voneinander separieren. Weil es sich bei beiden Effekten um eine analytische Unterscheidung handelt (Dollmann 2010), stellt den Ansatz vor methodische Probleme bei der Bestimmung ihres relativen Anteils an der Ungleichheitsentstehung. So heißt es, die Kritik am Kausalmodell zusammenfassend, bei Jackson (2013):

\footnotetext{
"The decomposition of IEO into primary and secondary effects rests on the principle that these effects act independently to produce overall inequalities. If students anticipate that they will not make an educational transition, they may then decide to work less hard and subsequently achieve a lower level of performance; this behavior violates the principle of independence of primary and secondary effects. If students do routinely make anticipatory decisions of this kind, the size of secondary effects estimated under the principle of independence would be an underestimate of the true role of choice in creating IEO.” (Jackson 2013: 17).
} 
Wie Neugebauer (2010) auf Basis bundesweiter Längsschnittdaten des DJI Kinderpanels in einer Dekompositionsanalyse zur Abschätzung der relativen Bedeutung beider Effekte zeigt, entfallen auf den Entscheidungseffekt 59 Prozent der am Übergang auf das Gymnasium beobachteten Bildungsungleichheit, wobei das Ausmaß dieses Effekttyps in Bundesländern mit verbindlicher Grundschulempfehlung stärker ausfällt als in Kontexten, in denen der Elternwille keiner institutionellen Einschränkung unterliegt. ${ }^{4}$ Zudem variieren die geschätzten Erklärungsgewichte je nach verwendeten Daten, so dass nationale wie internationale Studien zum Teil zu gegenteiligen Ergebnissen kommen, den primären Herkunftseffekten also größeren Anteil zuschreiben (ebd;; Ditton et al. 2005; Ditton und Krüsken 2008). Allerdings gilt es bei dieser Einschätzung zu differenzieren. Für Subgruppen der Bevölkerung, wie Migrantenkinder, stellt der leistungsbezogene Effekt der Familienherkunft, bei einem für diese Gruppe typischerweise besonders hohen Aspirationsniveau, anteilig sogar den Ungleichheitsmechanismus dar (neben möglichen Effekten der Diskriminierung) (Gresch und Becker 2010).

Der Dekomposition sozialer Bildungsungleichheit in primäre und sekundäre Herkunftseffekte kommt eine wichtige sozialpolitische Bedeutung zu. Beispielsweise könnten finanzielle Hilfsmaßnahmen zur Reduzierung sekundärer Herkunftseffekte beitragen, indem ein Mehr an ökonomischen Mitteln die in unteren Sozialschichten schwerer wiegenden Bildungskosten tragbarer macht (Dollmann 2010: 18). Damit stünde, im Falle einer adäquaten sozialpolitischen Umsetzung, ein wichtiger Baustein zur Veränderung des Bildungsverhaltens unterer Sozialschichten zur Verfügung. Werden herkunftsspezifische Werthaltungen gegenüber höhere Bildung in Rechnung gestellt, müsste dieser notwendigerweise ergänzt werden um das Aspirationsniveau anhebende Maßnahmen, was an eine formale Weiter- bzw. Höherbildung bildungsfernerer Schichten geknüpft ist. Eine Erhöhung des Bildungskapitals müsste, soll der sekundäre Herkunftseffekt bei der Bildungswahl abgeschwächt werden, vermutlich zusätzlich begleitet werden von einer beruflichen Höherpositionierung, um die soziale Distanz zum System höherer Bildung zu verkürzen, welche sich, wie oben dargelegt, an der Distanz zwischen elterlichem Sozialstatus und für das Kind angestrebtem Bildungsabschluss bemisst. Solche Maßnahmen sind jedoch kostspielig und ihr Erfolg ungewiss.

Zur Reduzierung primärer sozialer Herkunftseffekte setzt die Sozialpolitik starke Hoffnungen in das System institutioneller Frühbildung (Jackson 2013: 6), das das Kind als Lernumwelt direkt erreicht sowie einen Zugang zu den Eltern herstellt. Eine weitere, im deutschen Kontext wenig Beachtung findende Maßnahme im Kampf gegen primäre Herkunftseffekte, stellt das für alle Kinder verpflichtende Programm zur Früherkennung gesundheitlicher Probleme und lernrelevanter Entwicklungsauffälligkeiten dar. Um Entwicklungsdefiziten vorzubeugen, zielt das Präventionsprogramm zudem auf eine Stärkung der Erziehungskompetenz,

\footnotetext{
${ }^{4}$ Neben dem Freiheitsgrad, den Familien beim Grundschulübergang institutionell in einigen Bundesländern zugestanden wird (für eine Übersicht siehe Neugebauer 2010), variiert die Bedeutsamkeit beider Effekttypen in Abhängigkeit institutioneller Rahmenbedingungen, wobei das Gewicht sekundärer sozialer Herkunftseffekte von weiteren Strukturgrößen des Bildungssystems abhängt, unter anderen von der laufbahnbezogenen Optionsvielfalt, Zeitpunkt und Anzahl der Bildungsübergänge (Hillmert 2008; Becker 2009).
} 
indem Informationen, Rat und Erziehungshilfen zu einem gesunden Aufwachsen vermittelt werden. Im Unterschied zum relativ populären und erfolgreichen Frühbildungssystem, liegen kaum Evidenzen zur Wirksamkeit der U-Untersuchungen im Schulkontext vor (MeyerNürnberger 2002). Wie im empirischen Teil der Arbeit gezeigt werden kann, hängen Lücken im Vorsorgestatus tatsächlich mit schlechteren Bildungschancen zur Einschulung und Bildungsergebnissen in der Grundschule zusammen, was für die Relevanz des Präventionsansatzes spricht. Im Folgenden stellt sich nun die Frage, ob Boudon's Argumentation zur Erklärung sozialer Unterschiede in der Bildung und im Bildungsverhalten auch auf soziale Ungleichheiten in der Gesundheit und hierauf bezogenes Verhalten übertragen werden kann.

\subsubsection{Herkunft und Gesundheit}

Analog zu Bildung eröffnet Gesundheit soziale Teilhabe und gilt als Resultat der Interaktion zwischen sozialen Prozessen und erblicher Veranlagung (Weber 1994: 195 f.). Boudon's Konzept kann somit auf den ersten Blick relativ einfach auf die soziale Strukturierung von Gesundheit bezogen werden, obgleich die Begriffe primäre und sekundäre soziale Herkunftseffekte nicht analog in der Gesundheitsforschung verwendet werden.

Im Sinne primärer sozialer Herkunftseffekte beeinflusst die familiäre Ausstattung mit ökonomischen, kulturellen und sozialen Ressourcen, vermittelt über differentielle Lebensbedingungen und Handlungsspielräume, die gesunde Entwicklung von Kindern. Ungünstige soziale Umwelteinflüsse begünstigen dabei soziale Differenzen im Gesundheitsstatus - wie die vorliegende Arbeit mit Blick auf sozialdifferentielle Risiken für a) eine Frühgeburt oder niedriges Geburtsgewicht sowie b) die Schulfähigkeit zum Zeitpunkt der Einschulung belegt. Im Sinne sekundärer Effekte der sozialen Herkunft auf Gesundheit spielen mit der sozioökonomischen Situation verknüpfte Gesundheitsdispositionen und Verhaltensweisen der Eltern in Bezug auf die Inanspruchnahme des Gesundheitsversorgungssystems eine zentrale Rolle. Je nach sozialer Schichtzugehörigkeit bestehen bedeutsame Unterschiede im alltäglichen Umgang mit Gesundheit (Herzlich 1973; Sperlich und Mielck 2003; Faltermaier 1994), welche sich mit Blick auf das Risikohandeln aber nur bedingt rationaltheoretisch begreifen lassen:

\footnotetext{
„Ob man Zigaretten oder Alkohol konsumiert, ist häufig gewohnheitsmäßig gesteuert und wird selten noch eigens im Hinblick auf mögliche Folgen reflektiert. $\mathrm{Ob}$ man in seiner individuellen Zeitplanung Entspannungs- und Regenerationsmöglichkeiten vorsieht, beruht selten auf einem rationalen Kalkül unter Abwägung körperlicher Langzeitfolgen. Ähnlich festgefahren sind in der Regel Verhaltensweisen wie körperliche Aktivität, Verhalten im Straßenverkehr und andere.“(Weber 1994: 197)
}

Auf den zweiten Blick fällt es somit im Vergleich zu Bildung schwerer, Gesundheit theoretisch als Entscheidung bzw. sekundären sozialen Herkunftseffekt zu konzeptualisieren, nicht zuletzt aus dem Grund, weil es zu Gesundheit als menschliches Grundbedürfnis im Allgemeinen keine erstrebenswerte Alternative gibt - im Unterschied zur herkunftsspezifischen Wahl eines kürzeren (weniger Kosten verursachenden) oder längeren (kostenintensiveren, anspruchsvolleren und daher auch unsichereren) Bildungsweges. Nichtsdestotrotz bestehen im Bereich Gesundheit im Sinne sekundärer sozialer Herkunftseffekte Analogien zum Bildungsbereich. Der Umgang mit Gesundheit und Krankheit wird maßgeblich vom sozialen, kulturel- 
len (und religiösen) Kontext beeinflusst (Faltermaier 2009: 49). So prägt die soziale Herkunft das subjektive Denken über Gesundheit, einschließlich hieraus folgender Verhaltensweisen: Untere Sozialschichten sehen nicht nur die Einnahme eines hohen Sozialstatus eher als eine Frage des persönlichen Glücks an (Boudon 1974: 21; Hyman 1953), sondern begreifen auch die eigene Gesundheit häufiger als Schicksal, auf das sie selbst nur wenig Einfluss und Kontrolle ausüben können (gesundheitsbezogenen Selbstwirksamkeits- bzw. Kontrollüberzeugungen, siehe Sperlich und Mielck 2003). In Abgrenzung zu solchen fatalistischen Gesundheitseinstellungen lassen sich gesundheitsförderliche Dispositionen (Präventionsneigung, ausgewogene Ernährung, minimaler Alkoholkonsum, ausreichend Bewegung, Verzicht auf Rauchen und sonstigen Substanzkonsum) eher intentional verstehen, obgleich sich die Formierung gesundheitsförderlicher Dispositionen - wie auch das Risikohandeln - im Zuge der Primärsozialisation vollzieht und damit stark vom soziokulturellen Kontext abhängt, so dass sich erlernte gesundheitsbezogene Orientierungen, Wahrnehmungen und Verhaltensweisen zum Teil dem Bewusstsein entziehen (Weber 1994: 197).

„Symptomaufmerksamkeit und präventive Einstellung sind Korrelate eines Orientierungsmusters, das sich als ,individuelle Zukunftsorientierung" beschreiben lässt. Eine solche Einstellung, für die etwa ein langfristiges Planen der eigenen Lebensführung zentral ist, resultiert aber hauptsächlich aus mittelschichtspezifischen Erziehungspraktiken. Angehörige unterer Sozialschichten legen demgegenüber mehr Wert auf gegenwartsnahe [eher kurzfristig wirksame] Techniken der Situationsbewältigung [wie etwa stressinduzierter Zigarettenkonsum]. Sie klassifizieren auch häufiger als Angehörige anderer Sozialschichten Krankheiten als ,Schicksalsschläge‘."(Weber 1994: 197)

Neben herkunftsabhängigen Gesundheitseinstellungen verweist dieses Zitat auf sozialschichtspezifische Zukunftsorientierungen, wie sie in ähnlicher Weise, obgleich mit stärkerer Betonung materieller Größen, auch die an Boudon anknüpfende Bildungsforschung in Bezug auf herkunftsspezifische Zeithorizonte bei der Planung von Bildungskarrieren thematisiert:

\footnotetext{
„Arbeiterschichten sind hierbei im Nachteil: Erstens erzwingt bei ihnen der Kostendruck einen kurzen Zeithorizont, wodurch sie von einer längeren Ausbildung an Universitäten abgelenkt werden. Zweitens schränken geringer eingeschätzte Erfolgswahrscheinlichkeiten den Zeithorizont auf kürzere und weniger anspruchsvolle Bildungswege ein.“ (R. Becker 2011: 114, sich auf Hillmert und Jacob 2013 beziehend).
}

Übertragen auf den Gesundheitskontext heißt das, dass neben herkunftsspezifischen Präferenzen für kurzfristig wirksame wie potentiell gesundheitsabträgliche Stressbewältigungsformen vermutlich auch materielle Restriktionen (Geld; indirekt: Zeit), welche das Ausüben langfristig gesundheitswirksamer Entspannungstechniken behindern, eine erklärende Rolle spielen. Von möglicherweise übergeordneter Bedeutsamkeit könnte sich in Fragen der Kindesgesundheit jedoch das Motiv des Statuserhalts erweisen. Für diese These spricht die in oberen sozialen Schichten eher überlegte (geplante) Familiengründung, welche in Erwartung des Eintritts der Schwangerschaft schon Wochen und teils Monate vor der Empfängnis mit der Umstellung der gesundheitlichen Lebensführung vorbereitet wird, mit dem Ziel, verhaltensbedingte Gefahren für das gesundheitliche Wohl des sich in utero entwickelnden Embryos bzw. heranreifenden Fötus von vornherein auszuschließen. In diesem Sinn beinhaltet die Verantwortung der (werdenden) Mutter jedes Risiko für das ungeborene Leben zu vermeiden, um dem 
Nachwuchs einen bestmöglichen Start ins Leben zu ermöglichen. Obere Bildungsschichten entwickeln aufgrund umfangreicherer Wissensbestände zu Schwangerschaft und Geburt vermutlich realistischere Vorstellungen zu verhaltensgebundenen Komplikationen, welche die Entwicklung des ungeborenen Kindes gefährden (zur Schichtspezifik gesundheitsbezogener Wahrnehmungsschemata, Abel 2009 und Faltermaier 1994). Dass der Grundstein für den späteren Kompetenz-, Bildungs- und Statuserwerb bereits im Mutterleib gelegt wird, dürfte in den vorausschauenden Überlegungen zum frühen Verzicht auf Substanzkonsum oder der Inanspruchnahme der Schwangerenvorsorgeuntersuchungen, welche von werdenden Müttern aus oberen Bildungsschichten im Mittel frühzeitiger und in der Regel auch kontinuierlicher genutzt werden (Tietze et al. 1982), bewusst oder unbewusst eine Rolle spielen. Analog kann für die sozial selektive Nutzung der U-Untersuchungen für Kinder argumentiert werden.

Schließlich ist im Sinne sekundärer sozialer Herkunftseffekte denkbar, dass die Gesundheit von Kindern beim Eintritt in das Bildungssystem ein zentrales Entscheidungskriterium darstellt. Je nach sozialer Positionierung könnten Eltern schulpflichtiger bzw. schulpflichtig werdender Kinder die physische und psychische Entwicklungsbiographie des Kindes als Entscheidungskriterium für den Zeitpunkt des Schuleintritts heranziehen und bei der Frage Früh-, Regel- oder Späteinschulung differentielle Bewertungen vornehmen. Bei gleichem Entwicklungsstatus, schlechtem wie guten, könnten in Abhängigkeit der Schichtzugehörigkeit unterschiedlich ambitionierte Schuleingangsentscheidungen getroffen werden. Ressourcenstärkere Oberschichteltern könnten gegenüber ressourcenschwächeren Schichten eher die ambitioniertere Früheinschulung anstreben und sich (je nach Grad der institutionellen Beschränkung des Elternwillens) auch eher über eine schulärztliche Empfehlung für eine Regeleinschulung hinwegsetzen. Denn entscheidungstheoretisch implizieren Ressourcenvorteile bessere Möglichkeiten zur Entwicklungsförderung, welche wiederum das subjektiv wahrgenommene Risiko für das Auftreten von Schwierigkeiten bei der Bewältigung der Transition in die Grundschule reduzieren. Oberschichteltern könnten also demnach das Risiko für schulanfängliche Schwierigkeiten als kalkulierbar bzw. beherrschbarer einschätzen. Ebenso denkbar ist, dass bei suboptimaler Schulfähigkeit Eltern aus oberen Sozialschichten, im Vergleich zu Eltern aus unteren Schichten, eher für eine Spät- statt Regeleinschulung optieren, um ein Jahr wertvolle Zeit für den Ausgleich schulfähigkeitsbezogener Nachteile zu gewinnen, so dass der Nachwuchs mit optimalen Voraussetzungen die Schullaufbahn und das Elternprojekt Statuserhalt antritt.

Zusammenfassend lässt sich festhalten, dass Bildungs- und Gesundheitsunterschiede zwischen verschiedenen Bevölkerungsgruppen als Ergebnis des Wechselspiels primärer und sekundärer sozialer Herkunftseffekte betrachtet werden können. Im Unterschied zu Bildung und Bildungsverhalten lässt sich gesundheitsschädigendes Verhalten jedoch mit Boudon weniger überzeugend rationaltheoretisch begründen, wie etwa mit Blick auf in unteren Schichten stärker ausgeprägtes Risikohandeln (Rauchen während der Schwangerschaft), das sich plausibler mit sozialisations- und habitustheoretischen Konzepten verstehen lässt. Weitere Kritikpunkte an Boudons Theorie beziehen sich zum einen darauf, dass keine tiefergehenden Erklärungen für die Entstehung schichtspezifischer Leistungsdisparitäten (primärer sozialer Herkunftsef- 
fekt) angeboten werden (Maaz 2006: 54). Zum anderen blendet Boudon die Meso-Ebene und damit den institutionellen Ungleichheitsbeitrag des Bildungswesens, wie ihn Bourdieu und Passeron (1971) unterstellen, aus (Becker 2011: 113).

Begibt man sich nun mit Bourdieu auf Suche nach den frühen Ungleichheitsmechanismen setzt diese aufgrund der existenziellen Abhängigkeit des Nachwuchses von der Fürsorge und den Lebensbedingungen der Eltern sinnvollerweise zunächst beim Elternhaus an und ist sodann auszuweiten auf das Unterrichtssystem, in dem Individual- und Familienmerkmale auf sozial differentielle Weise schulerfolgswirksam werden. Auf welche Weise Familie und Schule Lebenschancen strukturieren, wird nun im Folgenden mit Bourdieus gesellschaftstheoretischem Ansatz anhand seiner Grundformel „Struktur-Habitus-Praxis“ dargestellt.

\subsection{Bourdieus gesellschaftstheoretischer Erklärungsansatz}

Bourdieu versteht die Gesellschaft als einen durch Konflikt gekennzeichneten Schauplatz, auf dem Menschen um soziale Statuspositionen konkurrieren. Die aus dem sozialen Statuswettbewerb hervorgehenden Sieger streben danach, die einmal errungene, mit verschiedenen materiellen und immateriellen Profiten, wie Geld und soziale Anerkennung, verknüpfte Positionierung im sozialen Raum zu wahren, zu verbessern und möglichst in der Generationenfolge aufrechtzuerhalten, wobei letzteres ein mehr oder weniger schwieriges Unterfangen darstellt. In westlichen Gegenwartsgesellschaften wird die soziale Stellung der Elterngeneration nämlich nicht ohne Umweg über das Bildungssystem an die Kindgeneration vererbt. Der Nachwuchs aller Familien - ob sozial privilegiert oder nicht - muss in einem formal organisierten Rahmen um die wertvollsten Bildungszertifikate, die eine Gesellschaft als Zugangsvoraussetzung für angesehene Statuspositionen zu verteilen hat, kämpfen. Das sozial strukturierte Feld, in dem die je nach Familienherkunft unterschiedlich günstig aufgestellte Bildungskonkurrenz ausgetragen wird, ist die Schule (Bourdieu und Wacquant 1996: 133). In Abhängigkeit der elterlichen Sozialstellung betreten Grundschulkinder mit ungleich verteilten lern- und schulrelevanten Kompetenzen, Fertigkeiten, Verhaltensweisen und familiären Unterstützungsressourcen im Rucksack die Bildungsarena, wo sie auf sozial differentielle Bildungshürden stoßen. Zur Veranschaulichung dieses Szenarios, bei dem mit unterschiedlichen Ressourcen ausgestattete Akteure um feldspezifische Profite ringen, sei Geißlers Ungleichheitsmetapher wiedergegeben, die „Bourdieus Vorliebe für die Sprache des Sports und Wettkampfs“ (Schwingel 1995: 83) adressiert und die Aufmerksamkeit auf den Ungleichheitsbeitrag der Schule lenkt.

„In der Konkurrenz um die höheren Bildungsabschlüsse gehen ungleich trainierte Gruppen an den Start, die unterschiedliche Ziele anvisieren. Die Trainingsrückstände werden dann während des Wettkampfes nicht aufgeholt. Da den schlecht Trainierten ihr Handicap bewußt wird, schrauben sie ihre Zielvorstellungen noch weiter zurück. Es fehlt ihnen während der Konkurrenz nicht nur an Unterstützung, Zuspruch und Durchhalteparolen aus dem Kreis der Familie und Freunde, sondern das Bildungssystem stellt ihnen auch noch zusätzliche Hindernisse in den Weg. So kommt es, daß sie auch bei gleichem Einsatz und gleicher Leistung nicht dieselben Ziele erreichen wie die Begünstigten. Vergleichsweise gut trainierte Gruppen mit Unterstützung ihrer näheren sozialen Umwelt auf glatten schulischen Bahnen wetteifern mit vergleichsweise schlecht trainierten Gruppen mit mangelnder Unterstützung auf schulischem Hindernisparcours.“ (Geißler 1994: 149) 


\subsubsection{Bourdieus analytischer Zugang - Struktur, Habitus und Praxis}

Um das Zustandekommen sozial ungleicher Bildungschancen zu erklären, entwirft Bourdieu ein kapital- und konflikttheoretisches Theoriegebäude. In diesem nimmt neben den individuellen und familiären Ressourcen, welche strategisch sinnvolle Bildungsmanöver ermöglichen oder einschränken, das Bildungswesen als strukturiertes Feld besonderer kultureller Praxen einen wichtigen Platz ein. Dem meritokratischen Ideal, wonach jedes Kind auf der Bildungsleiter nach oben steigen kann, wenn es sich nur genügend anstrengt (Leistungsprinzip) zuwiderlaufend, operieren die mit der Kulturvermittlung beauftragten Bildungsinstitutionen nach kulturspezifischen (Spiel-)Regeln und damit nur scheinbar demokratisch oder herkunftsneutral. Sozialer Aufstieg durch Bildung ist daher in den Augen Bourdieus und Passerons (1971) für den aus dem Arbeitermilieu stammenden Nachwuchs ein fast haltloses Versprechen, also nicht mehr als eine „Illusion der Chancengleichheit“, kurzum, ein „Mythos“ (Solga 2005), der als solcher aber nicht von der Arbeiterklasse entlarvt wird.

Mit hochkulturellem Kapital (Bildungskapital) ausgestatteten Bevölkerungsgruppen haben ein feineres Gespür bzw. praktischen Sinn dafür, was in der Schule verlangt oder nicht verlangt, erwartet oder nicht erwartet wird, möglich bzw. unmöglich ist, weshalb sich ihre Kinder aufgrund der relativen Vertrautheit mit den Inhalten der Unterrichtskultur relativ mühelos über die Leistungshürden hinwegsetzen und sich, besser angepasst an die praktischen Gepflogenheiten des Unterrichtssystems erwartungskonformer im Schulalltag bewegen, ohne bei den Lehrern und Lehrerinnen im kulturell-verhaltensbezogen Sinn anzuecken.

„Die Schulbildung orientiert sich aber so stark an der Elitekultur, daß ein Kind aus kleinbürgerlichem und mehr noch aus bäuerlichem oder Arbeitermilieu mühsam erwerben muß, was Kinder der gebildeten Klasse [mühelos von ihrem Elternhaus] mitbekommen: Stil, Geschmack, Esprit, kurz die Leichtigkeit und Lebensart, die dieser Klasse, da es ihre eigene Kultur ist, natürlich sind“ (Bourdieu und Passeron 1971: 42)

Vor diesem Hintergrund stellt die Schule den zentralen Kontext für die Reproduktion sozialer Ungleichheiten dar. Die Forschungsliteratur hält Befunde bereit, die belegen, dass sich Herkunftsunterschiede im vorschulischen Kompetenzerwerb in der Grundschule eher weiten als dass sie nivelliert werden (Becker 2011: 113; Diefenbach 2008; Ditton 2008).

Wie der Bildungswettkampf verläuft, das heißt, wie lange und mit welchem Ergebnis, ob mit schulischem Erfolg oder Misserfolg, ob mit einem niedrigen, mittleren oder hohen Bildungsabschluss oder gar ohne Abschluss, hängt dabei entscheidend von den ökonomischen, kulturellen und sozialen Ressourcen der Schülerinnen und Schüler und ihrer Familien ab, einschließlich von habituell bedingten, in der Schule mit praktischem Sinn eingesetzten herkunftsspezifischen Bildungs- bzw. Reproduktionsstrategien.

Für die Erklärung sozialer Ungleichheitsstrukturen erweitert Bourdieu den in der Ökonomie auf den materiell-ökonomischen Aspekt gesellschaftlicher Austauschbeziehungen reduzierten Kapitalbegriff, den des ökonomischen Kapitals (also Geld und alles in Geld Transformierbare) um zwei weitere Kapitalien: Kulturelles und soziales Kapital (Bourdieu 1983).

Diese Erweiterung wird der Ausdifferenzierung moderner Gesellschaften wesentlich gerechter, da hier das Prinzip der Eigennützigkeit nicht allein für den ökonomischen Warenaus- 
tausch reserviert, sondern auf weitere spezifische kulturelle, soziale und symbolische Praxisformen in ,nicht warenförmige[n] Ökonomien der verschiedenen sozialen Felder“ auszuweiten ist, in denen spezifische „Interessen, Einsätze und Profitmöglichkeiten“ vorherrschen, die von den Wirtschaftswissenschaften nicht erfasst werden (Schwingel 1995: 87).

Alle Ressourcen, welche für den Erwerb, Erhalt und Verbesserung des Sozialstatus (in verschiedenen Feldern) zweckdienlich sind, bezeichnet Bourdieu als Kapital (Bourdieu 1983), wozu neben ökonomischen, auch kulturelle und soziale Kapitalbestände fallen.

„Bourdieu verwendet den Kapitalbegriff für den Bereich kultureller und sozialer Ressourcen, weil er von der Grundannahme einer einheitlichen, Ökonomie der Praxis' ausgeht - einer strategischen Grundorientierung sozialen Handelns, die fernab eines ,rational-choice'-Kalküls darauf ausgerichtet ist, vorhandene Ressourcen weitgehend unbewusst so zu ,investieren', dass sie in Hinblick auf die Erzielung eines Maximums an symbolischen Kapital (,soziales Ansehen') und Erfolg bei der Weitergabe des eigenen sozialen Status an die nächste Generation (,soziale Reproduktion') eingesetzt werden.“(Georg 2005: 181).

Obwohl ökonomisches Kapital aufgrund „seiner Unersetzbarkeit“ (Müller 2014: 174) relativ dominiert, spielen bei der Erzeugung von Ungleichheiten in den Bereichen Bildung (Bourdieu 1992: 49 f.) und Gesundheit (Abel 2009; Williams et al. 1995) neben dem sozialen Beziehungskapital auch das Kulturkapital eine besonders wichtige Rolle. Was aber versteht Bourdieu unter kulturellem und sozialem Kapital? Soziales Kapital bezeichnet alle an eine soziale Gruppenzugehörigkeit gebundenen materiellen (instrumentelle Hilfen, wie z.B. Geld) und immateriellen Ressourcen (Wissen, Zeit, emotionale Zuwendung), deren Mobilisierung eine fortwährende Beziehungsarbeit vorausgeht (Bourdieu 1983: 191, 192).

Kultur- bzw. Bildungskapital kommt wiederum in drei verschiedenen Ausprägungsformen vor: Als verdinglichtes bzw. objektiviertes Kulturkapital (Museen, Bücher, Kunstwerke, Instrumente), in schulischen und akademischen Titeln, Zertifikaten oder Auszeichnungen institutionalisiertes Kulturkapital (sogenannte kulturelle Kompetenzausweise, Bourdieu 1983: 190, 1987: 51) und schließlich das, unter Ausschluss der Delegationsmöglichkeit, unter persönlichem Aufwand in familiären wie schulischen Lernumwelten einverleibte Kulturkapital. Letzteres umfasst eine nach der sozialen Klassenstellung willkürliche Bandbreite wissensund kompetenzbezogener Eigenschaften, mit denen sich objektivierte Kulturgüter symbolisch erschließen, genießen und beherrschen lassen (Bourdieu 1983: 189).

Das primär in der Herkunftsfamilie - erster ,,vergangenheitsloser“ pädagogischer Aktionsraum (Bourdieu 1973: 58), in dem Maßnahmen der Sozialisation und Erziehung mehr oder weniger bewusst bzw. planerisch zum Einsatz kommen (Bourdieu 1983) - und sekundär in außerfamiliären Kontexten, wie Kindertagesstätten und Schulen, inkorporierte Kulturkapital hängt maßgeblich mit dem Schulerfolg zusammen. Analog stehen und fallen die Chancen für ein gesundes Aufwachsen mit den materiellen Existenzbedingungen sowie den kulturellen Kapitalien (Gesundheitskompetenz), welches im Alltag neben der finanziellen Lage des Elternhauses und dort bereitgestellter sozialer Unterstützungsressourcen in besonderem Maße für die praktische Herstellung und Förderung der Gesundheit der Familienmitglieder bedeutsam ist und eng mit den habituellen Schemata zur Erfassung und Beurteilung gesundheitsbezogener Risiken sowie der Nutzung von Präventionsangeboten zusammenhängt (Abel et al. 
2009: 200, 201). Wie empirische Evidenzen zeigen, ist das Bildungskapital eng mit der Inanspruchnahme von Vorsorgediensten für Kinder assoziiert (Kurz und Becker 2017). Dieser Befund stützt die Annahme einer Schlüsselfunktion inkorporierten Kulturkapitals für die Ausübung gesundheitsorientierter Aktivitäten, welche sich mit herkunftsspezifischen gesundheitsbezogenen Selbstwirksamkeits- und Kontrollüberzeugungen habitustheoretisch herleiten lässt (Abel et al. 2009; Sperlich und Mielck 2003; Williams et al. 1995).

Verantwortlich für die Erzeugung sozialer Ungleichheiten im Erwerb von gesundheits- und bildungsbezogenem Humanvermögen sind also gesellschaftliche Verteilungsunterschiede im ökonomischen, kulturellen und sozialen Kapitalbesitz sowie hiermit verknüpfte Unterschiede in den bildungs- und gesundheitsbezogenen Orientierungen und Verhaltensweisen.

Mit Blick auf Gesundheit unterscheiden sich Menschen (als Angehörige sozialer Kategorien) darin, inwieweit sie ihrem Gesundheitsverhalten überhaupt Sinn zuschreiben und glauben, Einfluss auf ihre Gesundheit zu haben (Faltermeier 1994). In unteren Sozialschichten dominieren negative Konzepte (nach dem Verständnis: gesund ist, wer nicht krank ist) mit schwachen Kontroll- und Selbstwirksamkeitserwartungen, welche einer gesundheitsorientierten Lebensführung eher im Weg stehen (Sperlich und Mielck 2003). Mit Blick auf Bildung bestehen ebenfalls soziokulturell verschiedene Einstellungsmuster. Untere Sozialschichten schätzen beispielsweise den Wert akademischer Bildung geringer ein; im Vergleich zu höheren Schichten ist ihr Bildungsverhältnis eher instrumentell (Bourdieu 1983; Boudon 1974). ${ }^{5}$

Über die Zugehörigkeit zu einer der drei von Bourdieu unterschiedenen sozialen Klassen (Arbeiterklasse, Kleinbürgertum, Bourgeoisie) entscheiden Volumen und Struktur der drei ineinander konvertierbaren Kapitalsorten (Bourdieu 1987: 182) sowie der „Klassenhabitus, der ,normalerweise' (d.h. mit hoher statistischer Wahrscheinlichkeit) mit dieser Stellung verbunden ist.“ (S. 585). Wie dieses Zitat verdeutlicht, legt Bourdieu seine Gesellschaftstheorie ungleichheitssoziologisch als Ökonomie der Praxis bzw. (Alltags-)Praktiken an, wobei die Linien zwischen sozialen Klassenzugehörigkeiten auf der einen Seite und spezifischen Formen des Geschmacks und Lebensstils auf der anderen Seite zum zentralen Gegenstand der soziokulturellen Betrachtung werden (Müller 2014: 145).

Der Klassenhabitus verbindet dabei als ein von den ersten Prägeumständen abhängiges Dispositionssystem die soziale Ungleichheitsstruktur mit der Handlungsebene (Bourdieu 1987). Der Habitus wird gleichermaßen als Produkt wie Produzent gesellschaftlicher Ungleichheitsverhältnisse konzeptualisiert (Bourdieu 2013). Das heißt, einerseits handelt es sich beim Habitus um ein dauerhaftes System von Eigenschaften zur Wahrnehmung und Deutung der Sozialwelt. Dieses individuelle - kognitive, evaluative und motorische Dispositionssystem (Schwingel 1995: 65) - ist nach Bourdieu untrennbar mit den sozialen Umständen seiner Erstprägung, kurzum mit dem Elternhaus und den dort vorherrschenden sozialen Lebensbedingungen und kulturellen Alltagspraktiken verknüpft. Soziokulturell ähnlich strukturierte

\footnotetext{
${ }^{5}$ Bildungskonzepte variieren auch zwischen Fachdisziplinen: Naturwissenschaftlich betrachtet ist Schulfähigkeit Resultat biologischer Reifung - soziologisch das Ergebnis sozialer und biologischer Einflussgrößen.
} 
Kontexte des Aufwachsens erzeugen nach Bourdieu ähnliche Muster des Fühlens, Denkens, Wahrnehmens, Erkennens und Verhaltens. In diesem Sinne stellt der Habitus, „dessen Strukturen den Strukturen des [sozialen und kulturellen] Raumes entsprechen, in dem er sich hervorbrachte“ (Bourdieu 2013: 311), ein Produkt der Sozialwelt dar.

Zum Erzeuger gesellschaftlicher Strukturen wird der Habitus, indem er den sozialdifferentiellen Umständen seiner ursprünglichen Konstitution innerhalb der Familie entsprechend sozialdifferentielle Praktiken und Formen ihrer Ausübung hervorbringt - und zwar ,,jenseits aller expliziten Überlegungen und Reflexionen“" (Schwingel 1995: 64). Als Anschauungsbeispiel für den weitgehend unbewussten bzw. impliziten Praxisvollzug im Alltag gilt der habitualisierte herkunftsspezifische Umgang mit dem eigenen Körper (Bewegung, Ernährung, Pflege, Substanzkonsum), welcher klassenspezifische Körperformen und -haltungen sowie klassenspezifische Krankheits- und Sterbemuster hervorbringt.

Im anthropologischen Zentrum der Habitustheorie von Bourdieu steht somit ein Mensch, dessen kognitive und motorische Möglichkeiten zu handeln durch äußere Existenzbedingungen vorstrukturiert sind. Damit steht Bourdieus praktisches Handlungsprinzip ,in Opposition $\mathrm{zu}$ voluntaristischen Handlungstheorien, die das Prinzip des Handelns in den ,freien“ Entscheidungen der Akteure suchen“ (Schwingel 1995: 61) und somit in Opposition zum rationalen Handlungsprinzip, wie es Boudon vertritt. Zwar legt Boudon seine Handlungstheorie mithilfe der sozialen Positionstheorie von Keller und Zavalloni (1964) relational an, färbt aber das ihr zugrundeliegende Bild vom Menschen als ,,berechnende[s] Subjekt“ (Bourdieu 2013: 185) ökonomisch ein, was mit der Denkschule Bourdieus kontrastiert. Bourdieu kritisiert das in der Theorie rationalen Handelns gezeichnete Bild des „, frei, bewußt und, wie manche Utilitaristen sagen, with full understanding [agierenden Menschen], wobei die Handlung aus der Berechnung von Gewinnchancen hervorgeht“" (Bourdieu 2013: 177; Hervorhebung im Original), da diese Akteurskonzeption kurzerhand die Prägekraft der Gesellschaft für die Ausbildung individueller und sozialdifferentieller Handlungsdispositionen ausblendet.

\footnotetext{
„Was Lebewesen angeht, heißt das Dasein erworbener Dispositionen leugnen soviel wie das Lernen als selektive und dauerhafte Umwandlung des Körpers durch Stärkung oder Schwächung synaptischer Verbindungen zu leugnen." (Bourdieu 2013: 175)
}

Wie in den Mediationen dargelegt, braucht es nach Bourdieu (2013) den Dispositionsbegriff, um einer bestimmten anthropologischen Besonderheit Rechnung zu tragen, und zwar der natürlichen Konditionierbarkeit des menschlichen Körpers „nicht naturgegebene, [kulturell] beliebige Fähigkeiten zu erwerben“" (S.175). Die Sozialwelt dringt dabei über Maßnahmen der Sozialisation, Erziehung, Disziplinierung und Konditionierung in den Nachwuchs ein. Genauer schreibt sich die Gesellschaft - wie unauslöschliche Tätowierungen (Bourdieu 2013: 181) in den Körper ein und verwandelt damit auch die Sinne zum Erfassen und Verarbeiten von frühesten Umwelteinflüssen, wie sie in Gestalt des Erwerbs kognitiver Grundfähigkeiten im Zusammenhang mit den pädagogischen Arbeiten am (Vor-)Schulkind eine bedeutsame Rolle spielen, ,in Naturgegebenheiten“ (Bourdieu 2013: 181). 
„Die Welt ist erfassbar, unmittelbar sinnerfüllt, weil der Körper, der dank seiner Sinne und seines Gehirns fähig ist, auch außerhalb seiner selbst in der Welt gegenwärtig zu sein, von ihr Eindrücke zu empfangen und sich durch sie dauerhaft verändern zu lassen, über lange Zeit hinweg (seit seinem Ursprung) ihrem regelmäBigen Einwirken ausgesetzt war.“ (Bourdieu 2013: 174)

Es gilt mit Blick auf den Bildungserfolg also zu berücksichtigen, „das[s] Menschen [hier Schülerinnen und Schüler] keine abstrakten Geister oder ,Akteure', sondern - salopp gesprochen - auch aus Fleisch und Blut sind“ (Villa 2008: 201). Letzteres scheint angebracht, hält man sich vor Augen, dass in Schuleingangsuntersuchungen die Schulfähigkeit, welche anhand kognitiver wie körperlicher Eigenschaften, wie Größe, Gewicht und Motorik ${ }^{6}$ beurteilt wird, auf dem schulärztlichen Prüfstand steht. Diese durch äußere ökonomische Lebensbedingungen und kulturelle Erfahrungen erworbenen schulfähigkeitsbezogenen Größen formieren zusammen den klassenspezifischen Habitus einzuschulender Kinder als ein System bestimmter im Unterrichtssystem erfolgsrelevanter kognitiver (und motorischer) Dispositionen zum Erfassen, Ordnen, Beurteilen, Erinnern und verbal wie schriftsprachlich angemessenen Wiedergeben kultureller Lerninhalte (Bourdieu und Passeron 1971).

Überträgt man nun Bourdieus Gedanken zu Habitus und Einverleibung auf die Einschulungssituation, so verfügen die ,sozialen Akteure [hier: Vorschulkinder] über einen Habitus (..), den vergangene Erfahrungen ihren Körpern einprägten“ (Bourdieu 2013: 177). Einverleibungsprozesse basieren dabei auf der natürlichen Konditionierbarkeit des Körpers, auf sozialem Weg lern- und schulrelevante Fähigkeiten auszubilden: es gilt (Bourdieu 2013: 194): „Der Körper ist in der sozialen Welt, aber die soziale Welt steckt auch im Körper“. Und der sozialisierte Vorschulkörper wird, bevor der Startschuss für den Bildungswettlauf fällt, einer ersten ärztlichen Musterung seitens der Institution Schule unterzogen. Am Ende der Prüfung wird möglicherweise ein folgenreiches, ja eventuell demoralisierendes Urteil über den Habitus und seiner Trägerin gefällt: Schulfähig: Ja/Nein. Diese Eingangsselektion kann feldtheoretisch (Bourdieu und Wacquant 1996: 139, Hervorhebung im Original) als „Auswahl bestimmter [die Eintrittserwartungen erfüllender] Subjekte zuungunsten anderer" verstanden werden, wobei das „Recht auf Eintritt in ein Feld [hier: in die Grundschule] (..) durch den Besitz einer besonderen Konfiguration von [Habitus-]Eigenschaften legitimiert wird“.

Wie erfolgreich die Musterung ausgeht hängt dabei von der im Vorschulalter geleisteten kulturellen Einprägungsarbeit ab und damit entscheidend von der Zeit (Bourdieu 1983), die primäre pädagogische Akteure (Eltern) aufgewandt haben, um in der Kindergeneration die gewünschten und vom Unterrichtssystem (verkörpert in den sekundären Lehrinstanzen, der Lehrerschaft) erwarteten Dispositionen bis zum Beginn der Grundschule zu manifestieren.

In Erweiterung zu Bourdieu wird nun angenommen, dass die Transformierung objektiver Sozialstrukturen in habituelle Dispositionen nicht erst im Vorfeld der Einschulung sondern bereits viel früher, d.h. noch vor der Geburt beginnt. Es wird also unterstellt, dass analog zur

\footnotetext{
${ }^{6}$ „Die habituellen Dispositionen sind auf eine derart fundamentale Weise im Körper verankert, dass sie bis in die entwicklungspsychologisch grundlegende Schicht der motorischen Schemata reichen und dadurch die menschliche Existenzweise von Grund auf prägen.“(Schwingel 1995: 65)
} 
herausragenden Bedeutung der Bildungsdauer für die Einprägung kulturellen Kapitals die Dauer der intrauterinen Entwicklung (Schwangerschaftsdauer) womöglich von entscheidender Bedeutsamkeit ist, da die zur sinnlichen Erfassung der sozialen Welt zuständigen neurologischen Strukturen intrauterin angelegt und in Gestalt kultureller Praxen extrauterin objektiviert werden. Auch wenn der Bauch der (werdenden) Mutter einen wichtigen Schutzraum für den Nachwuchs und seine Entwicklung darstellt, ist dieser äußerst empfänglich für äußere potentiell entwicklungswidrige Einflüsse der Sozialwelt und somit zwangsläufig strukturiert und zwar in doppelter Hinsicht, da der Organismus schwangerer Frauen seinerseits früheren Einverleibungsprozessen und damit dem Risiko der sozialen Verletzung ausgesetzt war. ${ }^{7}$

„Mit einem heideggerisierenden Wortspiel könnte man sagen, daß die Disposition Exposition ist: Weil der Körper (in unterschiedlichem Ausmaß) exponiert ist, weil er in der Welt ins Spiel, in Gefahr gebracht wird, dem Risiko der Empfindung, der Verletzung, des Leids, manchmal des Tods ausgesetzt, also gezwungen ist, die Welt ernst zu nehmen (und nichts ist ernsthafter als Empfindungen - sie berühren uns bis ins Innerste unserer organischen Ausstattung hinein), ist er in der Lage, Dispositionen zu erwerben, die ihrerseits eine Öffnung zur Welt darstellen, das heißt zu den Strukturen der sozialen Welt, deren leibgewordene Gestalt sie sind.“ (Bourdieu 2013: 180)

\subsubsection{Einverleibung der Gesellschaftsordnung durch soziale Exposition}

Von Anbeginn seiner Existenz ist der Organismus mehr oder weniger günstigen Einflüssen der Sozialwelt exponiert. Dieser Umstand birgt Risiken für das Individuum wie für die familiäre Weitergabe kulturellen Kapitals. Bourdieu bleibt, was den Startzeitpunkt für die intergenerationale Transmission betrifft, relativ unkonkret. Hinsichtlich der Frage, wann der Organismus zu leben beginnt, findet sich mit dem Verweis auf „,seinen Ursprung“ eine vage auslegungsbedürftige Formulierung (Bourdieu 2013: 174). Der Ursprung des Organismus wird hier auf die vorgeburtliche embryonale Entwicklungsperiode näher eingekreist und medizinisch auf ungefähr zwei Wochen nach der Empfängnis festgelegt. Entwicklungsbiologisch wie soziologisch markiert dieser Zeitpunkt während der Schwangerschaft den Beginn der intergenerationalen (Gesundheits- und womöglich auch Bildungs-)Transmission, an welchem der embryonale mit dem mütterlichen Blutkreislauf, über welchen wichtige Nähr- aber auch potentiell entwicklungsschädigende Mikrostoffe zum Ungeborenen vordringen, verschaltet wird.

Potentielle Beeinträchtigungen ergeben sich auch für die sich intrauterin ausbildenden physiologischen Grundlagen des Habitus, also der herkunftsspezifischen „Systeme von Wahrnehmungs-, Bewertungs- und Handlungsschemata“ (Bourdieu 2013: 177). Der Habitus wird dabei nicht erst nach der Geburt, sondern bereits im Mutterleib durch die Sozialwelt vorstrukturiert. Angewandt auf die Schwangerschaft, dem Zeitfenster, in welchem in weniger als einem Jahr ein Mensch heranreift, stellen materielle Existenzbedingungen und die gesundheitliche Lebensführung der (werdenden) Mutter ein erhebliches Risiko für das ungeborene Leben

\footnotetext{
${ }^{7}$ Empirisch häufen sich die Hinweise, dass Schwangere, welche selbst als Frühgeborene zur Welt kamen, ein signifikant höheres Frühgeburtsrisiko haben (Razum et al. 2011). In diesem Befund spiegelt sich die grundlegende Idee der bourdieuschen Körpersoziologie, nämlich dass der sozial imprägnierte Organismus nicht vergisst und die soziale Frühgeburtsproblematik als Mehr-Generationen-Prozess ein soziologisch zu erklärendes Problem der sozialen Produktion und Reproduktion gesundheitlicher Ungleichheiten darstellt.
} 
dar, was in Abschnitt 2.4.2 sozialepidemiologisch mithilfe des lebenslauftheoretischen Modells kritischer Perioden im Detail zu spezifizieren sein wird.

Zusammenfassend ist festzuhalten, dass in Erweiterung zu Bourdieu, welcher den Anfang des Einverleibungsprozess auf den Zeitpunkt der Geburt datiert, eine Ausdehnung des intergenerationalen Transmissionsprozesses im Sinne einer zeitlichen Vorverlagerung angebracht scheint. Reproduktionstheoretischer Hintergrund hierfür sind Effekte der Frühexposition des Subjekts zur Sozialwelt, die nicht erst mit der Geburt, sondern in der vorgeburtlichen Lebensbzw. Entwicklungsphase auftreten. Es wird somit körpersoziologisch angenommen, dass die Sozialstruktur der Schwangeren sprichwörtlich „unter die Haut geht“ (Villa 2008: 215) und dabei bis zum im Uterus heranreifenden Kind vordringt, was im Kapitel zum Lebensverlaufsansatz berichtete Evidenzen zu ökonomischen und kulturell-verhaltensbezogenen Effekten auf das Geburts- bzw. Schwangerschaftsergebnis, so die recht technischen Begriffe für den Ausgang der Schwangerschaft bzw. die Gesundheit des Neugeborenen, nahelegen.

Nach der Geburt vollziehen sich durch den im Mutterleib sozial vorstrukturierten Körper mithilfe physiologisch im Gehirn lokalisierter habitueller Schemata schließlich Lernpraxen (Bourdieu 2013: 173), über welche kulturelles Kapital inkorporiert wird. Das Produkt des Einverleibungsprozesses ist ein im Schul- wie späterem Berufsalltag wirksames Dispositionssystem, das „im Alltag von Beobachtern [z.B. Lehrern, Schulärzten) als ,natürlich“, als quasi ,naturwüchsig“ wahrgenommen wird“" (Georg 2005: 181) - obwohl die Inkorporierung kultureller und im Unterrichtssystem sanktionierter Dispositionen an die soziale Herkunft rückgebunden ist. In einem der Klassenlogik nach operierenden Bildungssystem - welches über „Definitionsmacht“ für unterrichtsrelevante kulturelle Wissensbestände, Fähigkeiten und Verkehrsformen verfügt (Georg 2005: 181), würden dann vor allem jene Kinder schulische Erfolge einfahren, die über zur Schulkultur passende kulturelle und körperliche Dispositionen verfügen, also zumeist in obere soziale Klassen (termin-)geborene Kinder. Wie gestalten sich nun die Bildungskarrieren von zu früh geborenen Kindern?

$\mathrm{Zu}$ dieser Frage kann zunächst festgestellt werden, dass überproportional häufig in ressourcenärmere (Migranten-)Familien geborene Frühchen zur Entfaltung ihres Leistungspotentials zusätzliche Lernimpulse benötigen, jedoch im Elternhaus eher weniger Anregung und im Klassenraum zu wenig Beachtung finden: Eltern und Lehrkräfte adressieren die besonderen Lernbedürfnisse von Frühgeborenen vermutlich wissensbedingt nicht adäquat (Johnson et al. 2015). Jäkel et al. (2012: 150) verweisen in diesem Punkt auf normative Erwartungen seitens des Lehrkorpus und der Eltern:

„Viele Eltern und Lehrer erwarten, dass ehemalige Frühgeborene den Entwicklungsrückstand zu Gleichaltrigen bis zum Schuleintritt aufholen. Während dies auf die Mehrheit zutrifft, haben Frühgeborene mit hohem Entwicklungsrisiko oft noch langfristig Probleme, da sie eine eingeschränkte Plastizität besitzen und weniger von Lerngelegenheiten profitieren können.“ (ebd.)

Hinter diesen Überzeugungen steht ein an Schulreife und nicht an Schulfähigkeit orientiertes Entwicklungskonzept, das außer Acht lässt, dass sich Schulfähigkeit nicht ohne Zutun Dritter einstellt. So nimmt mitunter auch ärztliches Personal ein Auswachsen früher Entwicklungsde- 
fizite an (Kynø et al. 2013). Dabei benötigen Frühgeborene neben aufwendigen kulturellen Transmissionsanstrengungen der Eltern oftmals auch sonderpädagogische Zuwendung, um eine reelle Chance zu haben, lebensanfängliche Entwicklungsrückstände bis zur Einschulung aufzuholen (Wolke und Meyer 2000). Andernfalls ergeben sich in nach Noten selektierenden Bildungssystemen automatisch Bildungsnachteile. Diese auf die Interaktion zwischen pränatalen und institutionellen Kontextfaktoren abstellende Erklärung adressiert die Passung zwischen entwicklungs- und lernbezogenen Besonderheiten mit entwicklungsnormativen Erwartungen der „Gate-Keeper“, darunter schulärztliches Personal, das in der Schuleingangsuntersuchung ein Urteil über die Schulfähigkeit fällt oder das Grundschullehrpersonal, welches Übergangsempfehlungen an das Lern- und Leistungsverhalten der Schülerschaft knüpft.

Welche allgemeinen institutionellen Mechanismen nach Bourdieu (und Passeron) im Unterrichtssystem am Ungleichheitswerke sind, wird nun im Folgenden aufgezeigt.

\subsubsection{Der Ungleichheitsbeitrag des Unterrichtssystems}

Bourdieu zufolge tragen in modernen Gesellschaften die mit der Bildung von Kindern beauftragten Institutionen - entgegen der bildungspolitischen Hoffnung Chancengleichheit zu fördern - zur Reproduktion kultureller Ungleichheitsstrukturen und, da Kulturkapital auf Märkten in Geld konvertierbar ist, sozioökonomischer Verteilungsungleichheiten bei. So fungiert das Unterrichtssystem nicht nur als Kulturvermittler, sondern auch als Übermittler von sozialen Privilegien, indem es „hinter dem Mantel der Neutralität“ (Bourdieu 1973: 93) mit einer schichtspezifischen Auslese dafür sorgt, dass das „kulturelle Kapital wieder zum kulturellen Kapital wandert“ (ebd. 96). Die Reproduktion kultureller Kapitalunterschiede zwischen den sozialen Klassen erfolgt durch institutionelles Unterlassen. So stellt die Schule nicht oder nicht in methodisch angemessener Weise die Instrumente zur Aneignung der kulturellen Lerninhalte bereit, setzt gleichwohl aber ihren individuellen Besitz voraus (Bourdieu 1973: 103). Den während der pädagogischen Kommunikation im Klassenraum eingeprägten und in Leistungskontrollen überprüften Lehrstoff können somit in der Regel nur jene Schülerinnen und Schüler voll und damit zur vollsten Zufriedenheit der Lehrkräfte erfassen, die im Vorfeld der Schule mit der „legitimen“ Kultur und entsprechender Praktiken vertraut gemacht wurden - also aus der „kultivierten“ oberen sozialen Klasse stammende Schüler. Aus dieser Perspektive wird das Unterrichtssystem zum Monopol derjenigen Familien, welche es aus eigener Kraft, das heißt „mit eigenen Mitteln“ schaffen, die zur Aneignung der herrschenden Kultur notwendigen Instrumente an ihren Nachwuchs weiterzugeben (Bourdieu 1973: 103).

Primär zuständig für die pädagogische Vorarbeit ist nach Bourdieu die Familie, welche in Eigenregie, zumeist unbewusst (Bourdieu 1983) und in Abhängigkeit der ökonomischen, kulturellen und sozialen Kapitalausstattung mehr oder weniger früh und kontinuierlich lernrelevante „Investitionen an Zeit, Mühe und Geld“ (Bourdieu 1973: 106) in die Akkulturation des Kindes tätigt. Schülerinnen und Schüler, die die relativ komplexen (Sprach-),Codes“ (1973: 102) bzw. kulturellen Interpretationsschemata zur symbolischen Entschlüsselung von künstlerischen, literarischen oder historischen Werken besitzen und beherrschen, demonstrieren im 
Durchschnitt auch bessere Leistungen, was der Schulbetrieb mit guten Noten, günstigen Beurteilungen, ausdrücklichen Empfehlungen für weiterführende Schulen sowie mit der Vergabe gesellschaftlich angesehener Bildungszertifikate entsprechend positiv sanktioniert.

Erfolg im Bildungssystem basiert also nach Bourdieu auf der Passung zwischen familiärer Herkunfts- und Unterrichtskultur, weshalb sich besonders jene Gruppe schulisch im Nachteil befindet, deren Familie die größte kulturelle Distanz zum Unterrichtssystem aufweist - in Klassengesellschaften demnach Kinder der Arbeiterklasse. Die Ungleichheitsreproduktion über das Bildungssystem erweist sich dabei als umso „,vollkommener, je näher die Kultur, die es übermittelt, der herrschenden Kultur steht und je ähnlicher die Einprägungsweise, derer es sich bedient, der familiären Einprägungsweise ist.“ (Bourdieu 1973: 103). In der Konsequenz zieht am Ende der Schulzeit nur eine Minderheit der Arbeiterfamilien eine positive Erfolgsbilanz, wofür ein sozial verzerrtes Bewertungsverhalten der Lehrerschaft mitverantwortlich ist. Die Bildungschancen des Nachwuchses aus sozial unterprivilegierten Familien werden also auch unabhängig von den demonstrierten Leistungen und Fähigkeiten institutionell beeinträchtigt. Privilegierte Kinder aus sozial begünstigten Elternhäusern werden jedoch nicht mit Vorsatz bevorteilt. Vielmehr fließen in die Bewertung schulischer Leistungen, Begabungen und Ambitionen von Lehrern und Lehrerinnen, welche mehrheitlich selbst sozial begünstigen Klassen entstammen, ,mehr oder weniger unbewusst und ungeplant [der Lebens- und Erfahrungswelt höherer Schichten entsprechende] Kriterien, Wertorientierungen und sozialpsychologische Mechanismen [der Anpassung an Leistungserwartungen der Lehrkräfte] hinein, die sich aufsummieren und schließlich die Kinder aus der Mittelschicht tendenziell begünstigen.“ (Geißler 1994: 147). So unterliegen ungleich milde Notenvergaben und Empfehlungen für weiterführende Schulen einer Reihe schichtspezifischer Verzerrungen (für einen Überblick, Ditton 2005), so dass Arbeiterkinder, um im Bildungssystem erfolgreich zu sein, sogar ,ein Übersoll an Leistungen erbringen [müssen]“ (Geißler 1994: 146).

Neben der an Leistungskriterien sowie leistungsfremden Verhaltenskriterien orientierten Fremdselektion weisen Bourdieu und Passeron (1971) auf einen weiteren Selektionsmechanismus hin: Den Prozess der Selbsteliminierung. So nehmen sich Arbeiterkinder selbst bzw. auf Wunsch ihrer Familien früher aus dem Bildungssystem, indem sie sich selbst bei ausreichenden Leistungen für weiterführende Bildungswege eher gegen diese entscheiden, was teils weniger bildungsorientierten Dispositionen, teils aber auch den weniger ermutigenden Bildungserfahrungen mit den verzerrten „Verdikten der Prüfungsinstanzen“ (ebd. 180) geschuldet sein kann. Vor diesem Hintergrund trägt also das Unterrichtssystem durch ein offiziell demokratisches Ausleseverfahren, das auf meritokratischen Kriterien individueller Leistung, Anstrengung und Bildungseifer, welche allesamt untrennbar mit der sozialen Herkunft verbunden sind, basieren soll, faktisch jedoch einer klassenspezifischen Selektionslogik folgt, zur intergenerationalen Reproduktion von Bildungsungleichheiten bei, und zwar nicht allein in Frankreich, sondern insbesondere in Deutschland, wo sich die Schule angesichts des - im internationalen Vergleich - hohen Ausmaß sozialer Selektivität „trotz aller Reformversuche 
[z.B. Einrichtung von Gesamtschulen] (...) auch heute noch weitgehend als ,Mittelklasseinstitution“ dar[stellt].“(Geißler 1994: 148).

Zusammenfassend lässt sich festhalten, dass Bourdieus kultur- und sozialkapitaltheoretische Annahmen zur Spezifizierung primärer sozialer Effekte der Herkunft auf den Schulerfolg tiefere Einblicke in kulturelle Transmissionsprozesse zwischen Eltern- und Kindgeneration ermöglichen (Sullivan 2001; Georg 2005). Auch in der Gesundheitsforschung gilt Bourdieus Reproduktionstheorie als fruchtbar, um zu verstehen, wie differentielle gesundheitliche Dispositionen und Praxen die Entstehung sozialer Gradienten im Krankheits- und Sterbegeschehen begünstigen (Abel 2009; Williams et al. 1995).

Neben diesen Meriten zeigt und klärt Bourdieu jedoch, so die hier kurz angerissene internationale Kritik (für einen Überblick Georg 2005), nur begrenzt auf, wie sich Sozialisationsprozesse und die damit verknüpfte Habitusgenese im Detail vollziehen (Maaz 2006: 66). Dieser zentrale Kritikpunkt macht sich jedoch nicht nur an theoretischen Aussparungen fest, sondern auch an einer „ungenügende[n] Modellierung des unterstellten Wirkungsmechanismus“ (Georg 2005: 188, Hervorhebung im Original) durch die Berechnung einfacher Assoziationsmaße, die Bourdieu statistisch halfen, die vermuteten Relationen zwischen der sozialen Klassenstellung auf der einen Seite und den Schulleistungen der Kinder sowie hochkulturellen Familienpraktiken auf der anderen empirisch zu unterfüttern: „Die dazwischenliegende Kausalkette (...) wurde von Bourdieu interpretativ geschlossen, was jedoch nicht als empirischer Nachweis im engeren Sinne angesehen werden kann" (Georg 2005: 188).

Probleme, welche mit der empirischen Modellierung und Messung der einzelnen kausaltheoretischen Glieder verknüpft sind, finden sich aber nicht nur bei Bourdieu, sondern auch bei jenen, die die von Bourdieu vorgeschlagenen sozialen Reproduktionsmechanismen belegen oder widerlegen möchten (für einen Überblick siehe Georg 2005 oder Maaz 2006). Ein weiterer aus dem bildungssoziologischen Bereich der Übergangsforschung stammende Kritikpunkt an Bourdieus reproduktionstheoretischen Ansatz bezieht sich berechtigter Weise darauf, dass sich dieser (gegenüber dem mikrosoziologischen Ansatz Boudons) nur begrenzt eignet, aufzuzeigen, wie in stratifizierten Bildungssystemen an institutionell festgelegten Verzweigungen herkunftsspezifische Bildungsentscheidungen getroffen werden, welche im Aggregat zu dem Phänomen einer sozial ungleichen Bildungsbeteiligung führen (Maaz 2006: 61; vgl. Dollmann 2010).

Des Weiteren werden Bourdieus Ausführungen zum Ungleichheitsbeitrag des Unterrichtssystems eher kritisch bewertet, da hierfür harte empirische „Belege fehlen, die zeigen, dass die institutionelle Wertordnung, die geforderten Sprachcodes und die Verkehrsformen in der Schule, die vordergründig an den Normen der Mittelschicht orientiert sind, auf sozial weniger begünstigte Gruppen diskriminierend wirken“" (Maaz 2006: 66).

Insgesamt werden schichtspezifische Effekte der Institution Schule in der Forschungsliteratur zu herkunftsspezifischen Bildungsungleichheiten somit uneinheitlich betrachtet. Auf der einen Seite steht die konflikttheoretische Position Bourdieus, gemäß welcher die Schule einen signifikanten Anteil bei der Reproduktion bzw. Verstärkung herkunftsspezifischer Bildungs- 
ungleichheiten trägt, während auf der anderen Seite dem Bildungswesen eine ,ungleichheitsminimierende Funktion“ (ebd.: 62) zugeschrieben wird (DiMaggio 1982).

Auch wenn dieser Widerspruch hier nicht aufgelöst werden kann, kann die unter anderem durch die PISA-Studien belegte Fortdauer der sozialen und ethnischen Differenzen im deutschen Bildungswesen (Baumert et al. 2003; Georg 2005) sowie die Befunde zu sozial differentiellen Notenvergaben (Ditton 2005) Anlass für konflikttheoretische Problemperspektiven geben. Obgleich die Strukturmuster nicht unbedingt konflikttheoretisch gedeutet werden müssen, können sie zumindest angesehen werden als Beleg dafür, dass die Schule nach wie vor vor inklusiven Herausforderungen im Zusammenhang mit soziokultureller (Diefenbach 2011) und auch gesundheitlicher Heterogenität steht (Wolke und Meyer 1999).

Gerade für das Verständnis gesundheitsbezogener Nachteile und ihre potentiell negativen Folgen für den Bildungserfolg kann Bourdieus analytischer Zugang weiterhelfen. Insbesondere seine in den Mediationen (Bourdieu 2013) enthaltenen - obgleich zugegebenermaßen recht abstrakten (Müller 2014: 38) - Überlegungen zum Erwerb des Habitus eröffnen den Zugang zu einem von der Bildungsforschung bislang unbetretenen interdisziplinären Pfad der intergenerationalen Transmission von Lebenschancen zum Lebensbeginn. Das Potential der Reproduktionstheorie Bourdieus ist somit bei Weitem nicht ausgeschöpft.

Im nachfolgenden Kapitel wird dieser auf frühbiographisch wirksame Umweltbedingungen abstellende körpersoziologische Reproduktionsmechanismus, den Bourdieu (2013) auf die (recht einfache) Gleichung Disposition = Exposition bringt, mithilfe des sozialepidemiologischen Lebenslaufansatzes weiter spezifiziert und plausibilisiert.

\subsection{Lebensverlaufsperspektiven auf Gesundheit und Bildung}

Aus der Lebenverlaufsperspektive werden Entstehung und Wandel sozialer Ungleichheiten in den Lebenschancen über die gesamte Lebensspanne erforscht. Zweifelsohne stellen der Erwerb von Bildung und Gesundheit längerfristige Prozesse dar, welche erst im biographischen Kontext angemessen zu betrachten sind. In der bildungssoziologischen Lebenslaufforschung spielen prä- wie postnatale Lebensabschnitte bislang nur eine marginale Rolle bei der Erklärung herkunftsspezifischer Entwicklungs- und Bildungsverläufe. In der vorliegenden Schrift wird diese Rolle mit Retrospektivdaten genauer untersucht. Aufgrund von Datendefiziten können allerdings keine vollständigen Biographien nachgezeichnet, dafür aber für den Erwerb von Gesundheit und Bildung neuralgische Punkte bzw. Stationen im kindlichen Lebenslauf in den Blick genommen werden. Dabei wird detailliert darauf einzugehen sein, wie soziale Ungleichheiten in der Elterngeneration mit gesundheits- und bildungsbezogenen Ungleichheiten in der Kindergeneration zusammenhängen. Nachfolgend werden in diesem Kapitel zunächst allgemein Lebenslaufperspektiven auf Bildung und Gesundheit dargestellt (Abschnitt 2.4.1), bevor mit zwei Ansätzen der sozialepidemiologischen Lebenslaufforschung theoretisch spezifiziert wird, wie es zur Strukturierung von Lebenschancen vor wie nach der Geburt kommt. Letzteres erfolgt in zwei separaten Theorieabschnitten, in denen das Modell lebenslaufkritischer Entwicklungsperioden (2.4.2) sowie das Akkumulationsmodell vorgestellt wird (2.4.3). 


\subsubsection{Lebensverläufe als endogene Kausalzusammenhänge}

In der Soziologie wird der Lebenslauf als ein Mosaik von Übergängen verstanden, die einerseits sozialen Normierungen unterliegen, wie institutionelle Beschränkungen elterlicher Entscheidungsfreiheiten in Fragen der Bildung (Schulformwahl am Ende der Primarstufe) und Gesundheit (Besuch gesetzlich geregelter Vorsorgeuntersuchungen für Kinder), andererseits aber vom entwicklungsbiologischen Status abhängen (Maaz et al. 2006: 299). Biologische und soziale Statusübergänge werden bislang kaum aufeinander bezogen. Im Fokus des Kapitels stehen daher Interdependenzen zwischen den Dimensionen Gesundheit und Bildung.

Aus der Lebensverlaufsperspektive wird Gesundheit als über den gesamten Lebenslauf veränderliche Prozesskategorie verstanden (Faltermaier 2009: 46), je nach positivem oder negativem Vorzeichen der Umwelteinflüsse als Gewinn- oder Verlustgröße. In diesem dynamischen Sinn stellt Gesundheit Kapital dar, das in frühen Lebensphasen auf Basis genetischer und sozialer Faktoren gebildet, unter Aufwand im Säuglings-, Kleinkind- und Vorschulalter akkumuliert und durch Alterungsprozesse wieder abgebaut wird, wobei „Umfang und Tempo" des Kapitalabbaus von sozialen und individuellen Bedingungen im Erwachsenenalter moderiert werden (Power und Kuh 2008: 54). ${ }^{8}$ Mangels Verfügbarkeit an Geburtskohortendaten hat der Lebenslaufansatz erst zur Jahrhundertwende Eingang in die Gesundheitsforschung gefunden (Richter und Hurrelmann 2016: 264). Gegenüber dem englischsprachigen und skandinavischen Forschungsraum ist das Forschungsfeld in Deutschland kaum entwickelt (Dragano und Siegrist 2009: 191). Letzteres gilt auch im Vergleich zur bildungsbezogenen Lebenslaufforschung, deren Anfänge bis in die 80er Jahre des 20. Jahrhunderts zurückreichen (für Westdeutschland, Blossfeld 1988; Mayer 1991; Meulemann 1990; Henz und Maas 1995; für Ostdeutschland, Solga 1997). Analog zu Gesundheitskapital unterliegt auch Bildungskapital frühen Akkumulations- und späteren Verfallsprozessen (Bourdieu 1983). Zentraler Befund der Untersuchung von Bildungsverläufen verschiedener Geburtskohorten war eine - entgegen der bildungspolitischen Hoffnung in die Nivellierung sozialer Bildungsungleichheiten im $\mathrm{Zu}$ ge des massiven Ausbaus des Sekundarschulsystems (Bildungsexpansion) - lediglich moderate Abnahme der Chancenungleichheit in der Kohortenabfolge, welche sich hauptsächlich für den Erwerb mittlerer Bildungsabschlüsse nachweisen ließ (Solga und Becker 2012: 14). Zwischen oberen, mittleren und unteren Sozialschichten ungleich verteilte Chancen auf den Besuch eines Gymnasiums bestanden fort - ein heute noch substanzieller Ungleichheitsbefund.

Die jüngere bildungsbezogene Lebenslaufforschung knüpft an die Forschungsgegenstände und Methoden der älteren Lebenslaufstudien an, etwa mit Blick auf die Untersuchung ungleicher Chancen beim Zugang zur Hochschule (Neugebauer und Schindler 2012) oder den (sozi-

\footnotetext{
${ }^{8}$ Gesundheitskapital bezeichnet nach Power und Kuh (2008: 54) „die in früheren Lebensphasen ererbte oder erworbene Anhäufung von biologischen Ressourcen, welche den aktuellen Funktions- und Gesundheitsstatus determinieren. Dies schließt (...) die Widerstandskraft (Resilienz) gegenüber möglichen, auch zukünftig wirkenden Umweltstressoren mit ein. Der Begriff des Gesundheitskapitals hat eine gewisse Verwandtschaft mit dem früher üblichen Terminus der körperlichen Konstitution.“
} 
al selektiven) Besuch von Berufs- und Fachgymnasien, welche eine ,zweite“ Chance auf einen zur Hochschule führenden Abschluss eröffnen (Winkler 2017). Je nachdem, welche soziale Gruppe diese zusätzlichen Bildungschancen verstärkt nutzt, kann sich das an vorherigen Bildungsübergängen (wie dem Grundschulübergang) festgestellte Ungleichheitsausmaß ändern, das heißt zu- oder abnehmen oder aber unverändert fortbestehen.

Hierin liegt nach Hillmert (2008) die Notwendigkeit Bildungsungleichheiten im individuellen Lebensverlauf zu untersuchen, was nicht zuletzt mit kausaltheoretischen Überlegungen begründet wird: „Denn der zu einem bestimmten Zeitpunkt bei einer Person beobachtete Bildungsstatus lässt sich nicht unbedingt aus aktuellen Bedingungen erklären. Entscheidend ist oft die individuelle Vorgeschichte (...)“(ebd.: 83), die Zugangschancen zu institutionalisierten Lebensbereichen ermöglicht (Schule). Dabei wirken frühere biographische Ereignisse und Erfahrungen oftmals mit zeitlicher Verzögerung auf spätere vom Individuum zu bewältigende Statusübergänge ein. Der Lebensverlauf setzt sich somit zusammen aus einer Vielzahl nicht isoliert zu betrachtender biographischer Phasen und Statuspassagen (Mayer 1987).

„Der Lebensverlauf ist auch ein endogener Kausalzusammenhang. Spätere Ergebnisse, aber auch Zielsetzungen und Erwartungen, sind zu verstehen und zu erklären aus Bedingungen, Entscheidungen, Ressourcen und Erfahrungen der vorausgegangenen Lebensgeschichte“ (Mayer 1990: 10 f.)

In Anlehnung an Mayers Konzeption des Lebensverlaufs als endogenen Kausalzusammenhang wird davon ausgegangen, dass Schulerfolg nicht vom Entwicklungsstatus zum Zeitpunkt der Geburt (Übergang zwischen intrauteriner und extrauteriner Lebensphase) abzukoppeln ist. Je nach gesundheitlicher Ausgangslage, die entscheidend vom Timing der Geburt geprägt wird, entwickeln sich Kinder im Vorfeld der Einschulung wahrscheinlich unterschiedlich gut. Lebenslauftheoretisch lässt sich dies mit Elder's Vorstellung von Übergängen als mehr oder weniger abrupte Zustandswechsel (Elder 1985: 31 f.) verknüpfen, wobei „off-time Übergänge“ - wie in der vorliegenden Arbeit ein Frühstart ins Leben (also der Geburt vor Vollendung der 37. Schwangerschaftswoche, die die medizinisch-normative Grenze darstellt, bei deren Unterschreitung die allgemeine Lebensfähigkeit abnimmt) - negative Entwicklungsverläufe in der Kindheit begünstigen (Elder 1998, zit. n. Sackmann und Wingens 2001: 20).

Verlaufsungleichheiten treten, sofern nicht schon bei Schwangerenvorsorgeuntersuchungen oder U-Untersuchungen beobachtet, spätestens bei der anthropometrischen Vermessung und kognitiven Testung schulpflichtiger Kinder in der Schuleingangsuntersuchung zutage. Selbige dient als Beispiel für die Verwobenheit individueller Gesundheit und Kompetenzen mit dem System organisierter Bildung. So wirft der physische, psychische und soziale Entwicklungsstand von Kindern primär ein schulärztliches und sekundär ein innerfamiliär auszuhandelndes Problem der Entscheidung für eine Regel-, Spät- oder Früheinschulung auf. Durch das schulfähigkeitsbezogene Normraster fallende Jungen und Mädchen, darunter überproportional Kinder aus unteren Sozialschichten und Migrantenfamilien (und vermutlich Frühgeborene), werden häufiger ein Jahr vom Schulbesuch zurückgestellt, während den schulfähigkeitsbezogenen Entwicklungsnormen gerecht werdende Kinder die Einschulungsschwelle zumeist beim 
ersten Anlauf nehmen (Gomolla und Radtke 2002). Wie sind nun soziale mit der Schwangerschaft und Geburt verknüpfte Chancennachteile zu erklären?

In frühkindlichen Lebensphasen wie in vorgeburtlichen Entwicklungsabschnitten generierte Chancenungleichheiten werden bislang vor allem in der sozialepidemiologischen Lebenslaufforschung, welche mit der von David Barker (1991) vorgelegten Studie „The foetal and infant origins of inequalities in Britain“ eingeläutet wurde, im Kontext verhältnis- und verhaltensgebundener Risikoexposition untersucht (Dragano 2007: 18f). Er und sein Team fanden heraus, dass untergewichtige Neugeborene im Erwachsenenalter überdurchschnittlich häufig vorzeitig an Herz-Kreislauf-Erkrankungen versterben, ein von Nachfolgestudien in und außerhalb Großbritanniens bestätigter Ungleichheitsbefund. Lebensanfängliche Entwicklungsund Gesundheitsnachteile verlaufen dabei - analog zum Krankheitsgeschehen im Alter - entlang vertikaler sozialstruktureller Kerndimensionen, wie Bildung, Einkommen und berufliche Stellung, sowie, mit diesen Strukturmerkmalen konfundiert, auch entlang der ethnischen Herkunft als horizontale Ungleichheitsdimension (Behrman und Butler 2007; Razum et al. 2011).

Neben Effekten auf der Individualebene strukturiert der Kontext Gesundheitsverläufe, wie das Gesundheitssystem oder regionale Unterschiede in ärztlicher Versorgung, Therapie- und Fördermöglichkeiten (Mielck 2005). Soziale Gesundheitsdifferenzierungen werden daher also als Folge des Zusammenspiels verhältnis- und verhaltensbezogener mit biologischen Einflüsse während der Schwangerschaft und nach der Geburt verstanden (Power und Kuh 2008: 47).

Zusammenfassend interessieren in der gesundheits- wie bildungsbezogenen Lebenslaufforschung die familiale Weitergabe von Lebenschancen, einschließlich der mit frühen Entwicklungsbeeinträchtigungen assoziierten Konsequenzen für die spätere Gesundheit (Power und Kuh 2008: 46), den Kompetenzerwerb und Bildungserfolg (für primäre Herkunftseffekte, Jackson 2013: 13 und Kristen 2008: 231). Für die lebenslauftheoretische Erklärung des Zusammenhangs zwischen sozialstruktureller Lage des Elternhauses und der individuellen Gesundheit haben sich zwei nicht in Gänze überschneidungsfreie Modelle etabliert: Das Modell kritischer Perioden und das Akkumulationsmodell (Ben-Shlomo und Kuh 2002). Indem sie den sozialen Hintergrund als Abbild für relativ stabile soziale Kontextbedingungen verstehen, welche für die allgemeine Entwicklung des (ungeborenen) Kindes bedeutsame Schutzräume und/oder Risikolagen erzeugen (Power und Kuh 2008: 55), lassen sich diese Modelle auch auf soziale Ungleichheiten im Bildungserfolg anwenden.

\subsubsection{Modell kritischer Perioden: Schwangerschaft und frühe Kindheit}

Das Modell kritischer Perioden geht von einer Vorprogrammierung individueller Entwicklungsprobleme während der Schwangerschaft und früher Stadien der Elternschaft aus. In der Forschungsliteratur wird dann von einer kritischen Periode gesprochen, wenn der menschliche Organismus eine besonders störungsanfällige Entwicklungsphase durchläuft (Dragano 2007: 19). In diesen zeitlich begrenzten Perioden entstehende Schädigungen erweisen sich in der Regel als irreversibel bzw. permanent, wobei Langzeitfolgen oftmals erst zutage treten, wenn weitere gesundheitliche oder soziale Risikofaktoren hinzukommen. Nachfolgend wer- 
den zwei außerordentlich kritische Zeitfenster für die kindliche Entwicklung in das Blickfeld gerückt: Zum einen die Schwangerschaft als vorgeburtliche Entwicklungsperiode und zum anderen die ersten drei Jahre im Leben des Nachwuchses (frühe Kindheit).

\subsubsection{Die Schwangerschaft als entwicklungskritische Periode}

Im Mutterleib angelegte lebenszeitverkürzende chronische Erkrankungen des HerzKreislaufsystems oder des Stoffwechsels manifestieren sich häufig erst nach einer längeren Latenzphase im mittleren und höheren Erwachsenenalter: „der menschliche Körper [hat] offensichtlich ein gutes Gedächtnis: Schädigungen von Zellen und Organen, die im Mutterleib, im Säuglings- und Kindesalter erfolgen, können über Jahre und Jahrzehnte hinweg unbemerkt bleiben“ (Dragano 2007: 19). Relativ kurzfristige Spuren lassen sich hingegen in Gestalt sozialer Unterschiede im Reproduktionserfolg (Schwangerschafts- bzw. Geburtsergebnis) beobachten, wie sozialgruppenspezifische Prävalenzen für eine Frühgeburt, für niedriges Geburtsgewicht oder die Säuglingssterblichkeit belegen (Collatz et al. 1983; Jungbauer-Gans und Kriwy 2004: 10; Becker und Stolberg 2013; Jäkel et al. 2013; Razum et al. 2011).

Diese Befunde unterstreichen, dass es sich bei der Schwangerschaft um ein kritisches Zeitfenster der fötalen Entwicklung handelt. Während der pränatalen Wachstumsperiode läuft die Transmission entwicklungsungünstiger Einflüsse indirekt über den Organismus der werdenden Mutter (Siegrist 2005: 77). Wie die Forschung zeigt, reagiert der Fötus empfindlich auf Mangelsituationen (schlechte Hygiene, Wohn- und Ernährungsbedingungen), mütterlichen Stress (Partnerschaftsprobleme, soziale Isolation, fehlender sozialer Rückhalt) und eine gesundheitsriskante Lebensführung, wie die Exposition des Ungeborenen zu Schadstoffen durch Zigarettenkonsum (Steinhausen 2000: $101 \mathrm{ff}$; Ben-Shlomo und Kuh 2002; Dragano und Siegrist 2009: 185) - allesamt mit ökonomischen, kulturellen und sozialen Ressourcen der (werdenden) Mutter hochkorrelierende Risiken für perinatale Komplikationen, wie eine Frühgeburt oder niedriges Geburtsgewicht (Behrman und Butler 2007). Daneben begünstigt der Konsum von Alkohol, Drogen und Medikamenten Schwangerschaftskomplikationen, wobei diese Verhaltensmaße nicht eindeutig sozialstrukturiert sind.

Unabhängig von sozialen Verhältnis- und Verhaltensgrößen können Schwangerschaftskomplikationen zudem biologisch programmiert sein, im Zusammenhang mit Plazentainsuffizienz, Nabelschnurkomplikationen, genetischer Prädisposition für Fehlbildungen oder Behinderungen oder Hochrisikoschwangerschaften mit Mehrlingen, die mehrheitlich vorzeitig und einhergehend damit überdurchschnittlich häufig mit niedrigem Geburtsgewicht sowie organischen Unreifen zur Welt kommen (zu medizinischen Risikofaktoren, siehe im Überblick Bühling und Friedmann 2009). Ungefähr ein Drittel aller Frühgeburten wird in Folge einer Indikation (z.B. Mangelernährung) medizinisch eingeleitet. Spontane Frühgeburten werden mehrheitlich durch Komplikationen, wie etwa vorzeitige Wehen (40-45 Prozent aller Frühgeburten) oder einen Blasensprung (ca. 30 Prozent) ausgelöst (Urech et al. 2012: 5).

Steht dem Fötus nur ein Bruchteil der sonst üblichen Zeit zum Heranreifen zur Verfügung, fällt das Risiko für Schädigungen des Gehirns - „Denkzentrum des Menschen und (..) physio- 
logische Grundlage für seine besonders entwickelte Lernfähigkeit, zu Bewußtsein und zu gedanklicher Reflexion“ (Esser 1999: 153) - recht hoch aus.

\begin{abstract}
„Der Fötus durchläuft bis zur Geburt ein rasantes Wachstum, das in keiner anderen Lebensphase wieder erreicht wird. Für die Herausbildung bestimmter Organe gibt es zudem Zeitfenster, in denen ihre Entwicklung weitgehend abgeschlossen sein muss. Komplikationen [bedingt durch Unter- oder Fehlernährung, Substanzmissbrauch] können dazu führen, dass ein Organ oder ganze Gruppen von Organen oder funktionelle Mechanismen nicht zur vollen Reife gelangen. Solche Wachstumsstörungen bestehen dann lebenslang, daher wird in diesen Fällen auch von einer ,biologischen Programmierung' gesprochen“"(Dragano 2007: 19).
\end{abstract}

In bildgebenden Verfahren zeigen sich organische Unreifen des Denkapparates an einem bis zu 60 Prozent geringeren Faltungsgrad des Kortex, wobei dieser neurologische Nachteil insbesondere bei extrem frühgeborenen Kindern (Geburt vor der 28 Schwangerschaftswoche) „kaum aufholbar zu sein [scheint]“ (Bolten 2012: 8) und eine Reihe koordinations-, konzentrations-, sprach- und lernbezogener Schwierigkeiten (Behrman und Butler 2007) sowie internalisierender (Ängste, Depressionen) und externalisierender Verhaltensauffälligkeiten (ADHS, ADS) begünstigt (Chu et al. 2012). Solche, zu schulischen Erfolgsnachteilen führende neurologische Probleme stehen teilweise im Zusammenhang mit frühen Hirnverletzungen.

\footnotetext{
„Im Fall einer Frühgeburt ist das Hirn unreif und unterliegt zudem einer spezifischen Verletzungsgefahr durch eine mangelnde Nährstoff- und Sauerstoffversorgung oder eine vermehrte Blutungsneigung. Daraus folgt ein erhöhtes Risiko für atypische Hirnentwicklungsverläufe, die sowohl die Substanzbildung als auch die Verschaltung von Synapsen und Ausbildung von neuronalen Regelkreisen betreffen können. Es gilt die Annahme: ,Je unreifer das Gehirn zu Beginn des extrauterinen Lebens, desto atypischer seine strukturelle und funktionale Ausformung ${ }^{6}$. Andererseits setzt die verfrühte Reizexposition per se möglicherweise auch Entwicklungsprozesse in Gang, die frühgeburtstypische Reifungsmängel kompensieren und die Hirnorganisation befördern können. Diese Annahme wird unterstützt durch die Beobachtung, dass ein Frühgeborenes, das an seinem ursprünglich errechneten Geburtstermin bereits ein, zwei oder mehr Monate des extrauterinen Lebens hinter sich gebracht hat, manchmal in einigen Funktionsbereichen weiter entwickelt ist als ein reifes Neugeborenes.“ (von der Wense und Bindt 2013: 134)
}

Aber auch die Gruppe der späten zwischen der 33 und vor Vollendung der 37. Schwangerschaftswoche entbundenen Säuglinge, bei denen in der Regel wenige oder keine organischen Verletzungen zu erkennen sind (von der Wense und Bindt 2013: 135), fällt später häufiger durch kognitive und verhaltensbezogene Beeinträchtigungen auf (zum Überblick, de Jong et al. 2012; Sucksdorff et al. 2015; keine Unterschiede finden z.B. Harris et al. 2012). In der Literatur besteht Konsens, dass ein Frühstart ins Leben mit einer langfristig erhöhten Vulnerabilität im kognitiven (und weniger im motorischen) Funktionsbereich einhergeht, wobei Frühgeborene in Messungen der Intelligenz im Mittel circa 2 bis 3 Punkte hinter Reifgeborenen zurückliegen. Obgleich dieser Unterschied auf den ersten Blick wenig bedeutsam für den Bildungserfolg erscheint, schneiden Frühgeborene schulisch im Mittel schlechter ab als Reifgeborene. Als Grund hierfür gilt die „Häufung von subtilen kognitiven Beeinträchtigungen und Teilleistungsstörungen“" (von der Wense und Bindt 2013: 133), welche dazu führen, dass für die Lösung von Aufgaben insgesamt mehr Anläufe und somit mehr Zeit gebraucht wird.

Zusammenfassend liefern neuropsychologische Studien erste Hinweise zur Relevanz vorwie auch nachgeburtlicher Umweltfaktoren für die Ausbildung kognitiver Potenziale (Jackson 2013: 13). In Bezug auf postnatale Einflüsse gilt die Zeitspanne der frühen Kindheit als kritisch für wachstumsbezogene wie kognitive Entwicklungsprozesse (Dragano 2007: 20). Im 
mehrere Jahre umfassenden Zeitraum zwischen Geburt und Schuleintritt treten eine Vielzahl sozialer Umwelteinflüsse in Interaktion mit der Gesundheit und prägen auf komplexe Weise den Kompetenzerwerb des Nachwuchses. Das Modell kritischer Perioden hilft nun dabei, die Genese sozialdifferentieller Entwicklungsverläufe in der frühen Kindheit besser zu verstehen. Die frühe Kindheit gilt unter kognitiven, neurologischen und verhaltensbezogenen Aspekten als kritische Periode.

„Cognitively, experience is sequential: Experiences in infancy establish habits of seeking, noticing, and incorporating new and more complex experiences, as well as schemas for categorizing and thinking about experiences. Neurologically, infancy is a critical period because cortical development is influenced by the amount of central nervous system activity stimulated by experience. Behaviorally infancy is a unique time of helplessness when nearly all of children's experience is mediated by adults in one-to-one interactions permeated with affect." (Hart and Risley 2003: 9)

\subsubsection{Die frühe Kindheit als entwicklungskritische Periode}

Neben der Schwangerschaft adressiert das Modell kritischer Perioden auch mehr oder weniger förderliche Einflüsse während früher Stadien der Elternschaft. Obwohl die Fürsorge, Pflege und Lernstimulation von Kindern nicht an das biologische Geschlecht gekoppelt sind (Barnard und Solchany 2002) gelten den Nachwuchs umsorgende Aktivitäten kulturraumübergreifend als primär mütterliches Handeln. Dabei erweist sich die Qualität der MutterKind-Beziehung als bedeutsamer Entwicklungsfaktor, wobei sich ungünstige Bindungsqualitäten überproportional häufig bei Familien mit niedrigem Bildungsstand zeigen, da sich hier psychosoziale Belastungen konzentrieren (Bradley und Corwyn 2002; Lanfranchi und Burgener Woeffray 2013: 604). Diese teils sozioökonomisch mitbedingten Belastungen bereiten bei Nicht-Bewältigung den Boden für ein negatives Familienklima, das, vermittelt über Vernachlässigung und Gewaltanwendung, Kinder in der allgemeinen Entwicklung gefährden (Lanfranchi und Burgener Woeffray 2013: 604; Siegrist 2005: 190).

Als förderlich für die Bindungsqualität zwischen Mutter und Kind gilt ein positives emotionales Klima, das sich durch ein hohes Maß elterlicher Responsivität (soziale Ansprechbarkeit) und Reziprozität auszeichnet. In der vorsprachlichen Phase stellen diese sozialen Eigenschaften wichtige elterliche Basiskompetenzen im Umgang mit dem Kleinkind dar, um „ein Urvertrauen affektiver Konstanz und Sicherheit [zu schaffen] (Siegrist 2005: 191) - das Fundament für eine gesunde Entwicklung und für den Erwerb verhaltensbezogener und kognitiver Kompetenzen in den ersten drei Lebensjahren.

Von außerordentlich lernrelevanter Bedeutung erweist sich neben einem einfühlsamen auch ein kognitiv stimulierender Interaktionsstil. Familien in sozioökonomischen Risikolagen übernehmen in der vorsprachlichen Entwicklungsphase, in welcher die Interaktionsverantwortung primär bei den Eltern (und nicht bei den Kindern) liegt, im Schnitt seltener die Initiative zur didaktischen sprachbasierten Interaktion, welche sich durch ein vielfältiges, an den Fortschritten der kindlichen Entwicklung orientiertes Angebot an Lernobjekten und deren verbale Präsentation auszeichnet. Das Ausmaß der sozial differentiellen Sprachexposition bis zum Alter von 3 Jahren schätzen Hart und Risley (2003) auf eine (kumulative) Differenz von circa 30 Millionen Wörtern zwischen der höchsten und niedrigsten sozialen Statusgruppe, den Au- 
toren zufolge eine „early catastrophe“ Kinder in sozial weniger privilegierten Familien bekommen dabei in der Regel erst dann mehr elterliche Aufmerksamkeit, nachdem sie laufen und zu sprechen begonnen haben (Barnard und Solchany 2002).

Zwar machen Kinder durch wachsende Selbständigkeit neue Erfahrungen, allerdings prägen vergangene Stimulationsdefizite die Art und Qualität der selbstgewählten Lernerfahrungen (Hart und Risley 2003).

„Once children become more independent and can speak for themselves, they gain access to more opportunities for experience. But the amount and diversity of children's past experience influences which new opportunities for experience they notice and choose"(Hart and Risley 2003: 9).

Neben dem elterlichen Interaktionsverhalten gilt es bezüglich der frühen Bindungsqualität aber auch Neugeborene als Interaktionspartner in den Blick zu nehmen. Aus ungleichheitstheoretischer Sicht spielt die Gesundheit im Sinne der interaktionsbezogenen Funktionsfähigkeit des Nachwuchses eine wichtige Rolle. Wie die Säuglingsforschung zeigt, verfügen (gesunde) Kinder über kognitive und sozioemotionale Kompetenzen, welche dazu befähigen, von Geburt an individuelle Bedürfnisse (nach körperlicher Nähe, Interaktion, Nahrung) mitzuteilen und frühe Lernerfahrungen zu machen (Schlesiger et al. 2011: 188). Sozialstrukturell sind aber nicht nur die Fähigkeiten der primären Bezugspersonen zum reziproken Austausch ungleich verteilt, sondern auch interaktionsbezogene Kompetenzen und Kapazitäten Neugeborener. Im Vergleich zu Termingeborenen senden Frühgeborene im Durchschnitt weniger eindeutige Verhaltenssignale, was demzufolge ihre Entschlüsselung und adäquate Beantwortung durch die Eltern erschwert. Zudem verhalten sich Frühgeborene in sozialen Interaktionen häufiger passiv: Sie sind schwerer zur Interaktion zu motivieren, zeigen in Interaktionen eher kürzere Aufmerksamkeitsspannen und haben im Mittel eine geringere Toleranz gegenüber einer größeren Bandbreite elterlicher Stimulationsangebote (Barnard und Solchany 2002). Folglich können Interaktionen mit Frühgeborenen für Eltern mit größerem Aufwand verbunden sein. Gleichzeitig kann damit die Ausschöpfung interaktiver Belohnungen (z.B. in Gestalt des „soziales Lächelns“ Neugeborener) geringer ausfallen, was Unzufriedenheit im Erleben der Mutterrolle sowie psychosozialen Stress begünstigt (Barnard und Solchany 2002).

In diesem Kontekt kann angenommen werden, dass ein reziproker Austausch zwischen Eltern und Kind dann schwerer herzustellen sein wird, wenn beide Interaktionspartner geringe soziale (Interaktions-)Kompetenzen aufweisen, wie es beispielsweise häufiger bei Eltern-KindDyaden der Fall ist, bei denen sowohl eine sozioökonomische Risikosituation in der Familie als auch ein gesundheitliches handicap bei dem Kind vorliegt. Bei in interaktionsbezogener Hinsicht doppelt benachteiligten Mutter-Kind-Dyaden stellt sich in der Konsequenz häufiger eine Störung der „emotional symbiosis“ ein (Benedek 1959, zit. nach Barnard und Solchany 2002), was auf beiden Seiten - der Mütter wie der Kinder - für Unzufriedenheit sorgt.

Frühgeborene aus einkommens- und bildungsschwächeren Elternhäusern weisen vor diesem Hintergrund demnach potentiell in mehrfacher Hinsicht Startchancennachteile auf: Einerseits eine suboptimale entwicklungsbezogene Ausgangslage zur Geburt, andererseits die Hypothek, in ein sozial weniger privilegiertes Elternhaus geboren zu sein - ein für die vorschuli- 
sche Kompetenzentwicklung eher ungünstige Kombination (Jäkel et al. 2013). Aus sozialisationstheoretischem Blickwinkel bieten Risikofamilien im Mittel nicht nur weniger visuelle und auditive Anregungsmöglichkeiten zur Entwicklung kognitiver Fähigkeiten, sondern sehen sich zusätzlich aufgrund des besonderen Interaktionsverhaltens Frühgeborener besonderen Erziehungsanforderungen gegenüber. Insbesondere die spontane, nicht medizinisch ausgelöste Frühgeburt kommt in der Regel überraschend und geht bei Eltern, unabhängig von der Sozialschichtzugehörigkeit, mit einem hohen psychischen Belastungsgrad einher.

„They [parents of preterms] take on the role of parent before they or their baby are ready for it. They do so under highly stressful conditions with limited opportunities for normal interaction, and many find their babies difficult to manage." (Goldberg und DiVitto 2002: 335).

Die adäquate Bewältigung der vergleichsweise hohen Erziehungsanforderungen in Familien mit Frühgeborenen setzt spezifisches Erziehungswissen voraus, über das Eltern aus unteren Sozialschichten im Mittel weniger verfügen (Barnard und Solchany 2002). Des Weiteren gelten Mütter und Kinder aus Risikofamilien eher als weniger sozial ansprechbare Interaktionspartner: Mütter mit formal geringer Bildung reagieren im Mittel weniger kontingent (d.h. seltener unverzüglich und angemessen mit positivem Affekt und verbalen Stimuli) auf das Verhalten des Kindes; gleichzeitig sind (frühgeborene) Kinder aus Risikofamilien weniger sozial ansprechbar, d.h. sie reagieren häufiger gereizt, sind schwieriger zu beruhigen, lehnen Berührungen häufiger ab und lassen sich schwerer füttern (Barnard und Solchany 2002). Frühgeborenen fällt es vergleichsweise schwer, innere Erregungszustände zu regulieren, was wiederum die Eltern vor mentale Herausforderungen stellt.

„Die beschränkte Selbstregulationsfähigkeit vieler Frühgeborener stellt zudem erhöhte Anforderungen an die interpersonale Emotionsregulation durch die Eltern. Dies kann zu einer Überforderung und damit einhergehender Erschöpfung der primären Bezugspersonen führen, was sich wiederum negativ auf deren Fähigkeit zur co-regulatorischen Unterstützung des Kindes auswirken kann. Dadurch bedingte dysfunktionale Interaktionen können die Eltern-Kind-Beziehung dauerhaft beeinträchtigen.“ (Bolten 2012: 11)

Infolge einer posttraumatischen Fütterstörung lassen sich Frühgeborene weniger gut stillen, da sie im Zuge der Beatmung und Ernährung über die Sonde häufig ,,aversive Erfahrungen im Mund-, Schlund- und Rachenbereich“ erlebt haben (Bolten 2012: 9). Stillschwierigkeiten und mangelnder Kontakt zu den primären Bezugspersonen während der Hospitalisation erschweren das bonding zwischen Mutter und frühgeborenem Kind. Mit der geringeren Zufuhr von Muttermilch nehmen Frühgeborene im Mittel auch weniger für die Stärkung des Immunsystems und die Gehirnentwicklung wichtige Nährstoffe auf. Welche Konsequenzen erwachsen aus ungünstigen frühen Bindungserfahrungen für die Entwicklung von Kindern?

Werden kindliche Verhaltenssignale und Bedürfnisse nicht angemessen sozial beantwortet, also nicht harmonisch und vorwergnehmend befriedigt, leidet darunter das Urvertrauen und einhergehend damit die Ausbildung sozialkognitiver Fähigkeiten, wie beispielsweise die Fähigkeit zur sozialen Perspektivenübernahme und Empathie in sozialen Interaktionen. Unsicher gebundene Kinder sind ,im stärkeren Maße anfällig für chronische Stresserfahrungen in sozialen Interaktionen“ (Siegrist 2005: 195), was wiederum ihre Vulnerabilität gegenüber stress- 
bedingten Erkrankungen erhöht. Darüber hinaus werden bei diesen Kindern häufiger Lernbeeinträchtigungen beobachtet, die vermutlich mit einem weniger aktiven Explorationsverhalten des Kindes und einer weniger umfangreichen sowie später einsetzenden kognitiven Stimulierung zusammenhängen (vgl. Goldberg und DiVitto 2002: 336). Nichtsdestrotrotz können negative Entwicklungsverläufe durch eine gesteigerte (elterliche) Zuwendung und Anregung in eine positive Richtung gelenkt werden, sodass in vorgeburtlichen Entwicklungsperioden angelegte Nachteile durch soziale Umweltfaktoren in ihren negativen Effekten auf die kognitive Entwicklung ein Stück weit modifiziert werden können (ebd.).

\subsubsection{Kumulationsmodell}

Das Kumulationsmodell nimmt an, dass im Lebenslauf hinzukommende Risikoerfahrungen, die häufig in Verbindung mit einer materiellen Benachteiligung auftreten, frühbiographische Vulnerabilitäten negativ verstärken. Im Sinne sozialer Verursachungsketten, sogenannter „chains of risk“ (Dragano und Siegrist 2009: 189), bedingt die Anhäufung und Interaktion entwicklungswidriger Bedingungen in Familien aus unteren Sozialschichten Gesundheits- und Kompetenznachteile. Akkumulationstheoretisch kann dabei unter Zuhilfenahme der körpersoziologischen Überlegungen Bourdieu's argumentiert werden, dass die Einverleibung der sozialen Welt - Bourdieu zufolge ein nach der Geburt einsetzender Transmissionsprozess - einen vorgeburtlichen Ursprung hat, also noch bevor Eltern ihr Bildungskapital durch mehr oder weniger gezielte Sozialisationspraxen an ihren Nachwuchs weitergeben können.

Zur Plausibilisierung der These einer frühen Ungleichheitsverursachung können weitere kumulationstheoretische Konzepte angeführt werden. Nach dem Matthäus-Theorem („Wer hat, dem wird gegeben") kumulieren kleine (hier: lebensanfängliche) humanvermögenbezogene Ausstattungsunterschiede über den individuellen Lebensverlauf (frühe Kindheit) zu größeren (Kompetenz- und Entwicklungs-)Unterschieden (DiPrete und Eirich 2006), die in den U-Untersuchungen für Kinder oder in Einschulungsuntersuchungen nach außen sichtbar werden können. Mit dem Konzept des kompensatorischen Vorteils „as a stratifying mechanism that complements those of cumulative advantage and path dependence" (Bernardi 2014: 1) können zur Verstärkung sozialer Ungleichheiten unter Kindern mit gesundheitsbezogenen (Frühgeburt, niedriges Geburtsgewicht) oder bildungsbezogenen Nachteilen (z.B. junges Alter bei Schulbeginn) beitragende Bedingungen spezifiziert werden. Der Ansatz geht davon aus, dass höhere gegenüber unteren Sozialschichten (lebens-)anfängliche Nachteile durch kompensatorische Strategien ausgleichen können, wobei relative ökonomische, kulturelle und soziale Kapitalvorteile sowie das Statuserhaltmotiv zum Tragen kommen (Boudon 1998). Dabei variiert die Investitionstätigkeit nach Eintritt des Risikoereignisses in Abhängigkeit des Bildungskapitals (Almond und Mazumder 2013). Während formal hoch gebildet Eltern im Mittel mehr (kognitive Stimulations-)Zeit für Kinder mit anfänglichen Nachteilen (niedriges Geburtsgewicht) aufbringen, richten formal niedriger gebildete Eltern ihren Fokus verstärkt auf (Geschwister-)Kinder ohne Entwicklungsnachteile (Hsin 2012). Ersterer Gruppe gelingt es im Gegensatz zur zweiten Gruppe in der Konsequenz demnach (besser) die potentiell mit 
einem Risikoereignis assoziierten Langzeitfolgen, wie spätere Gesundheits- oder Bildungsnachteile, abzuwenden (Bernardi 2014: 5).

Im kumulations- und investitionstheoretischen Kontext kann darüber hinaus auch bei vergleichbarem (außer-)familiären Förderumfang von sozial differentiellen Entwicklungs-, Lernund Bildungsverläufen ausgegangen werden. So nimmt Kristen an (2008: 232), dass in Abhängigkeit der vorschulischen kompetenzbezogenen Ausgangslage ,identische [Bildungs]Investitionen zu divergierenden Lernergebnissen führen [können]“, das heißt genauer, in den Bildungserwerb von Kindern (mit entwicklungs- bzw. lernbezogenen Nachteilen) getätigte Investitionen können sich als unterschiedlich effizient herausstellen (ebd.).

Zusammenfassend kann zu den zwei lebensverlaufstheoretischen Modelltypologien festgehalten werden, dass der Einfluss einer aus entwicklungsbiologischer Sicht zu kurzen Schwangerschaft auf den Kompetenz- und Bildungserwerb höchstwahrscheinlich über a) entwicklungswidrige Prozesse während der kritischen intrauterinen Entwicklungsperiode sowie kritischer Entwicklungsphasen in der frühen Kindheit sowie in Ergänzung über b) Prozesse der Akkumulation früher gesundheitlicher und sozioökonomische Belastungen (Aufaddieren der Entwicklungsgefahren) vermittelt ist. Ergebnis dieser entwicklungswidrigen Prozesse sind soziale Entwicklungs-, Gesundheits- und Kompetenzunterschiede, die zu verschiedenen Zeitpunkten im Lebenslauf (Geburt, frühe Kindheit, Vor- und Schulalter) beobachtet werden.

Nachfolgend wird nun die im Theoriekapitel entlang ressourcentheoretischer und sozialepidemiologischer Perspektiven auf soziale Ungleichheiten in der Gesundheit und Bildung entwickelte Grundargumentation zusammengefasst.

\subsection{Zusammenfassung}

Die im Theoriekapitel dargelegten Konzepte und Erklärungsansätze gehen davon aus, dass Bildung und Gesundheit individuell im Lebenslauf erworben werden. Gemeinsam ist den Ansätzen die Annahme, dass Gesundheit sowie Kompetenz- und Bildungserwerb der Kindergeneration in erster Linie als familiäre Herstellungsleistungen zu interpretieren sind, nicht zuletzt aus dem Grund, weil der Elterngeneration die primäre Verantwortung für die Fürsorge, Sozialisation und Kindererziehung obliegt (Jungbauer-Gans 2006).

Das Hauptaugenmerk gilt dabei (werdenden) Müttern. Sie tragen nicht nur den Nachwuchs aus, sondern übernehmen in der Regel die Hauptverantwortung für seine Pflege, Gesundheit, Betreuung, Erziehung (Barnard und Solchany 2002) und (Früh-)Bildung (Geißler 1994: 143). Folglich interessieren in erster Linie mütterliche Eigenschaften und in zweiter - sozialkapitaltheoretischer - Linie väterliche Merkmale. Besondere Aufmerksamkeit verdient der ökonomische, kulturelle und soziale Ressourcenpool sowie, hiervon nicht unabhängig, das vor- wie nachgeburtliche Gesundheitshandeln und das Zurechtkommen mit der Elternrolle im Alltag.

Kapitaltheoretisch wird dabei vermutet, dass der gesellschaftliche Mikrokosmos Familie schon während der Schwangerschaft die Weichen für die lebensanfänglichen Gesundheitsund späteren Bildungschancen des Nachwuchses stellt, wobei die Herkunftseffekte vermutlich über verschiedene Mechanismen der pränatalen Exposition zur Sozialwelt vermittelt werden. 
Im Mittelpunkt der theoretischen Betrachtung steht folglich die fetal-origin-These, die eine lebensgeschichtlich frühe Ungleichheitsverursachung postuliert (Dragano 2007; Erhart et al. 2008). Reproduktionstheoretisch kann mit Bourdieu und sozialepidemiologisch mit dem lebenslauftheoretischen Modell kritischer Perioden angenommen werden, dass der ungeborene Nachwuchs im Mutterleib mehr oder weniger entwicklungsgünstigen materiellen Einflüssen (Armut) oder kulturell-verhaltensbezogenen Expositionen (Substanzkonsum) ausgesetzt und in diesem Sinne ganz und gar vom elterlichen Wohlergehen und Gesundheitshandeln abhängt.

Die existenzielle Abhängigkeit von elterlichen Ressourcen setzt sich sodann in den ersten Lebensjahren fort: So stehen und fallen die (Über-)Lebenschancen des Nachwuchses mit der sozialen Lage und psychosozialen Verfassung primärer Bezugspersonen (Esser 1999; Hurrelmann 2006). Soziologisch nimmt folglich die Herkunftsfamilie einen besonderen Stellenwert für die Erklärung sozial ungleicher Lebenschancen ein. Denn die Familie schafft je nach Ressourcenverfügbarkeit einen mehr oder weniger förderlichen Rahmen für einen komplikationsarmen Verlauf der Schwangerschaft, für einen guten Start ins Leben, ein gesundes und behütetes Aufwachsen im Elternhaus, eine an kognitiven Stimuli reiche Lernumwelt sowie für die individuell erfolgreiche Bewältigung schulischer Lern-, Leistungs- und Verhaltensanforderungen (Bourdieu 1983; Bradley und Corwyn 2002).

Für die soziale Strukturierung von Gesundheit und Bildung nimmt deshalb Bourdieus Reproduktionsannahme (1983) einen zentralen Stellenwert ein, der gemäß die ersten Transmissionsumstände die individuelle Aneignung humanen Vermögens entscheidend mitprägen. Die hieraus abzuleitende Grundannahme lautet, dass sich Prozesse der intergenerationalen Weitergabe gesundheitlicher und bildungsbezogener Privilegien nicht erst nach der Geburt vollziehen, sondern in der vorgeburtlichen Entwicklungsphase einsetzen und sich in nachgeburtlichen Lebensabschnitten fortsetzen (Jackson 2013; Jäkel et al. 2012; Bergmann et al. 2007; Becker und Biedinger 2006; Erhart et al. 2008; Kristen 2008; Wolke und Meyer 2000).

Folgt man diesem Grundgedanken, gilt es schwangerschaftsbezogene Mechanismen hinter primären sozialen (und ethnischen) Herkunftseffekten in der Gesundheit und im Schulerfolg in das theoretische Blickfeld zu führen und empirisch zu untersuchen. Während der Schwangerschaft wirksam werdende Ungleichheitsbedingungen lassen sich dabei, wie im Theoriekapitel aufgezeigt, in den Ansatz primärer wie sekundärer sozialer und ethnischer Stratifikationseffekte integrieren, indem sozioökonomische und soziokulturelle Herkunftsmerkmale als pränatale wie postnatale Entwicklungs- bzw. Lebensrisiken konzeptualisiert und deren Wirkung mit den sozialepidemiologischen Lebensverlaufsmodellen weiter spezifiziert werden. Im Sinne primärer Stratifikationseffekte beeinflussen neben ökonomischen und sozialen insbesondere kulturelle Ressourcen die Säuglingsgesundheit, den vorschulischen Kompetenzerwerb und die Bewältigung schulischer Anforderungen. Im Sinne sekundärer Herkunftseffekte spielen Eltern (und ihr Bildungskapital) im Rahmen vor- und nachgeburtlicher Entwicklungsinvestitionen, worunter auch das Präventivhandeln fällt, eine vermutlich entscheidende Rolle bei der intergenerationalen Transmission gesundheitlicher und bildungsbezogener Privilegien. 
Wie mit dem sozialepidemiologischen Modell sensibler bzw. kritischer Entwicklungsperioden verdeutlicht wurde, erweist sich beim Übergang zur Elternschaft vor allem eine niedrige Bildung der (werdenden) Mutter als Entwicklungsrisiko für (ungeborene) Kinder. An dieser Stelle sei noch mal ein kurzes Beispiel genannt: Untere Bildungsschichten planen Schwangerschaften seltener (Stolberg und Becker 2015; Raum et al. 2001; mit Blick auf den Übergang zum dritten Kind: Schneider 2016), was nicht nur die Wahrscheinlichkeit ihrer Unerwünschtheit erhöht, sondern darüber hinaus zu Stress führt und suboptimales Coping begünstigt (z. B. Rauchen). Ungeplant schwanger gewordene Frauen bemerken des Weiteren die Schwangerschaft zumeist später, was eine zeitlich später erfolgende Inanspruchnahme der Schwangerenvorsorgeuntersuchungen und Anpassung an einen gesundheitsorientierten Lebensstil mit sich bringt und somit ungünstige Schwangerschaftsresultate vorstrukturiert. Zum anderen wird im Kontext einer sozial differentiellen gesundheitsbezogenen Risikowahrnehmung die intrauterine Exposition zu Toxinen (Rauchschadstoffen) mit abnehmendem Bildungsgrad wahrscheinlicher (Currie und Moretti 2003), wobei Gruppen mit formal niedrigem Bildungsabschluss die vom Substanzkonsum ausgehende gesundheitsschädigende Wirkung eher unterschätzen.

Vor diesem ressourcen- und habitustheoretischen Hintergrund wird davon ausgegangen, dass die Güte materieller Lebensbedingungen und die hiervon mitabhängige gesundheitliche Lebensweise der Eltern (insbesondere der Mütter) die Güte der intrauterinen Umweltbedingungen prägt, unter denen das Ungeborene heranreift und die für die Entwicklung im Vorschulalter und erfolgreiche Bewältigung des Schulalltags von Bedeutung sind. Die schwangerschaftsbezogenen Lebensbedingungen bereiten demnach, so eine weitere expositionsbezogene Annahme, nicht nur die Basis für einen guten lebensanfänglichen Entwicklungsstatus, sondern ebenso die kognitiven Grundlagen für die Bildungsaneignung in (außer-)familiären Lernkontexten. Der Kompetenzerwerb wird im Vorschulalter durch frühe und kontinuierliche Entwicklungsinvestitionen strukturiert, wobei Eltern mit entsprechend hohem Bildungskapital familiäre Kulturationsanstrengungen vermutlich strategisch über den am Kindesalter gemessen relativ frühen Eintritt in Einrichtungen der Frühbildung (Kratzmann und Schneider 2009) und den zumeist lückenlosen Besuch der U-Untersuchungen abzusichern versuchen. Diese Annahme wurde kapitaltheoretisch in Bourdieus Ökonomie der Praxis eingebunden.

Die vorschulischen Investitionsmöglichkeiten im Elternhaus werden dabei begrenzt durch herkunftsabhängige Opportunitätsstrukturen, Betreuungspräferenzen und Präventionseinstellungen (Bradley und Corwyn 2002; Peter und Spieß 2015). Zudem variieren die Bildungserträge der Weitergabe kulturellen Kapitals in den Familien höchstwahrscheinlich nach der Schwangerschaftsdauer. Gegenüber Termingeborenen ziehen Frühgeborene aufgrund einer im Durchschnitt kognitiv eingeschränkteren Aufnahme- und Konzentrationsfähigkeit im geringeren Umfang Kapital aus häuslichen Lerngelegenheiten (Jäkel et al. 2012: 150). Frühgeborener Nachwuchs stellt in diesem Zusammenhang, so die investitionstheoretische Argumentation, besonders ressourcenarme (Migranten-)Familien vor insgesamt größere Herausforderungen ökonomisch (zur Finanzierung entwicklungskompensatorischer Maßnahmen), psychosozial 
(aufgrund unterschiedlicher Coping-Kompetenzen) sowie erzieherisch (aufgrund unterschiedlicher Erziehungskompetenzen).

In der Eltern-Kind-Dyade sind Frühgeborene zudem, so die Annahme aus dem Modell kritischer Perioden zu Stratifikationseffekten während früher Phasen der Elternschaft, schwierigere Interaktionspartner, die aufgrund entwicklungsbiologischer Besonderheiten (im Zusammenhang frühgeburtsbedingter organischer Unreifen und Verletzungen sowie negativer Erfahrungen infolge längerer Hospitalisationsphasen in reizarmen Umgebungen) im Vergleich zu Termingeborenen weniger eindeutig entschlüsselbare Verhaltenssignale senden und in Interaktionen mit den Eltern eine kürzere Aufmerksamkeitsspanne haben.

Wendet man sich der Elternseite zu, fordern formal höher gebildete Mütter, welche mit der Mutterrolle im Mittel besser zurechtkommen, den Nachwuchs häufiger zur Interaktion auf und reagieren auf dessen Verhaltenssignale angemessener (Kontingenz) (Goldberg und Divitto 2002). In unteren Bildungsschichten treffen also häufiger zwei für die Entwicklungschancen von Kindern ungünstige Bedingungen aufeinander: Eine im Mittel häufiger eingeschränkte Neugeborenengesundheit und eine häufiger vorzufindende sozioökonomische Risikolage der Eltern.

Aus dieser spezifisch in unteren Sozialschichten anzutreffenden Ungleichheitskonstellation erwachsen über Prozesse der Akkumulation entwicklungsbezogener Vor- und Nachteile häufiger Nachteile beim Erwerb kognitiver Grundfähigkeiten, welche sich im Bildungssystem in schulische Leistungs- und damit Notennachteile übersetzen. Wie Evidenzen belegen, kommen Frühgeborene im Vergleich zu Termingeborenen im Durchschnitt schlechter mit den alltäglichen schulischen Heraus- und Anforderungen zurecht (Wolke und Meyer 2000).

Nach dem Modell kritischer Entwicklungsperioden werden diese Nachteile während der Schwangerschaft und in der frühen Kindheit generiert. Im Kleinkindalter eingeschränkte interaktionsbezogene Aufmerksamkeits- und Aufnahmefähigkeiten begleiten Frühgeborene, so die lebenslauftheoretische Problemsicht, oftmals bis ins Grundschulalter, woraus sich vermittelt über spezifische Leistungs- und Verhaltensprobleme, schlechtere Chancen für die Positionierung in weiterführenden Schultypen ergeben. Erfahrungsgemäß gilt nach jetzigem Wissensstand: "The effect of infant health on educational success is non-trivial and cannot be explained away by controlling for SES [socioeconomic status]“" (Lynch 2011: 502).

Die Theoriediskussion kann schließlich wie folgt zusammengefasst werden: Soziologischer Dreh- und Angelpunkt zur Erklärung gesundheitlicher Ungleichheiten zum Lebensbeginn und ihrer potentiell negativen Bildungsfolgen sind zwei aufeinander zu beziehende Theoriestränge: Auf der einen Seite die klassische theoretisch elaborierte und empirisch gut untermauerte ressourcentheoretische Erklärungslinie nach Boudon und Bourdieu und auf der anderen Seite der soziologisch bislang kaum ausgeleuchtete sozialepidemiologische Erklärungsstrang, welcher schwangerschaftsbezogene Ursachen für das im Durchschnitt schlechtere gesundheitliche und schulische Abschneiden des Nachwuchses aus sozial weniger begünstigten (Migranten-)Familien ins Feld führt. 
Aus der bisherigen Argumentation lassen sich zwei Arbeitshypothesen zur gesundheitlichen Ausgangslage, einschließlich ihrer strukturierenden Bedeutung für den Schulerfolg ableiten:

1) Einerseits wird eine soziale und (aufgrund der Konfundierung von sozialem und Migrationshintergrund) ethnische Strukturierung der Gesundheitschancen zum Lebensbeginn erwartet (Hla). Ein Teil der Varianz in der Säuglingsgesundheit nach sozialen und ethnischen Merkmalen geht auf schwangerschaftsbezogene Größen zurück (H1b).

2) Andererseits wird eine soziale und ethnische Strukturierung der Bildungschancen erwartet (H2a). Ein Teil der gruppenspezifischen Schulerfolgsvarianzen lässt sich neben nachgeburtlichen Faktoren über schwangerschaftsbezogene Größen aufklären (H2b). 


\section{Ableitung der Forschungsfragen}

Aufbauend auf dem dargelegten theoretischen Hintergrund werden in diesem Kapitel die Forschungsfragen abgeleitet. Begonnen wird mit der Frage nach den Gründen für das Zustandekommen herkunftsbedingter Ungleichheiten im Geburtsergebnis (Abschnitt 3.1). Es folgt die Ableitung der Frage zu den Faktoren hinter den herkunftsspezifischen Präventionsdistanzen am Beispiel des selektiven Besuchs der U-Untersuchungen (Abschnitt 3.2). Schlusspunkt bildet die Herleitung der dritten Fragestellung, in deren Mittelpunkt schwangerschaftsbezogene Faktoren zur Erklärung herkunftsabhängiger Bildungschancen stehen (Abschnitt 3.3).

\subsection{Herkunftsbedingte Ungleichheiten im Geburtsergebnis}

Soziologisch kann die Schwangerschaft als ein sehr intimer, die bisherige Lebensführung umund den weiteren Lebensweg der werdenden Mütter vorstrukturierenden Lebensabschnitt verstanden werden. Medizinisch betrachtet stellt die in der Regel zehn Monate (40 Wochen) umfassende Schwangerschaft eine kritische Lebensphase dar - für die Schwangere wie für das ungeborene Kind (Dragano und Siegrist 2009; Ben-Shlomo und Kuh 2002). In diesem Kontext wirft die gesundheitliche Ausgangslage zum Zeitpunkt der Geburt ein soziales Ungleichheitsproblem auf, da sich Neugeborene in Abhängigkeit der familiären Herkunft bedeutsam im Entwicklungsstand (bemessen an der Schwangerschaftsdauer und damit zusammenhängend im Geburtsgewicht) unterscheiden (Collatz et al. 1983; Jungbauer-Gans und Kriwy 2004: 10; Milewski und Peters 2014). Welche mit der familiären Herkunft verknüpften Ressourcen- und Verhaltensmerkmale produzieren diese strukturierten Reproduktionsmuster?

Da diese Frage bislang nicht befriedigend beantwortet wurde, rückt die Arbeit soziale Disparitäten in der Schwangerschaftsdauer in den Fokus. Unter Rückgriff auf Bourdieus Kapitaltheorie gilt es herauszufinden, welche Bedingungen den Zusammenhang zwischen der elterlichen Bildung und dem Ereignis einer Frühgeburt hervorbringen. Spielen soziale Unterschiede in der Kulturkapitalausstattung und damit assoziierte Unterschiede in den gesundheitsbezogenen Einstellungen, Selbstwirksamkeits- und Kontrollüberzeugungen und Gesundheitspraxen eine Schlüsselrolle für die Entstehung sozial strukturierter Frühgeburtsprävalenzen? Welchen Beitrag leistet das soziale Beziehungskapital Schwangerer? Erweisen sich eine Partnerschaft (und daran gebundene Unterstützungs- und Kontrollprozesse) sowie regelmäßige Besuche durch Familie und Freunde als gesundheitswirksam?

Einerseits soll also geprüft werden, inwiefern der Zusammenhang zwischen (mütterlichem) Bildungsabschluss und dem Frühstart ins Leben durch ökonomische, soziale sowie lebensführungsbezogene Merkmale (Rauchen während der Schwangerschaft, Vorsorgeverhalten) vermittelt wird. Andererseits sollt im Hinblick auf die Moderation kultureller Kapitaleffekte auf die Frühgeburt getestet werden, ob das Bildungsgefälle in der Schwangerschaftsdauer durch die ökonomische und soziale Kapitalausstattung in seiner Steilheit variiert werden kann. 
Als zentrales, zwischen sozialer Stellung und Gesundheitspraxis vermittelndes Bindeglied kommt dabei der (Gesundheits-)Habitus nicht nur hypothetisch zur Anwendung, sondern wird über ein operationales Abbild in Gestalt dreier allgemeiner habitueller Konstrukte, darunter a) die persönliche Risikobereitschaft, b) die Überzeugung, das Leben ist abhängig von Glück und c) die Überzeugung wenig Kontrolle über das Leben zu haben, auf den Prüfstand gestellt. Damit besetzt die Arbeit eine offene Forschungslücke. Abbildung 3.1 stellt schematisch das zugrundegelegte Theoriemodell dar (siehe Kapitel 5 für eine differenziertere Version).

Abbildung 3.1: Erklärungsmodell zu herkunftsspezifischen Geburtsergebnissen

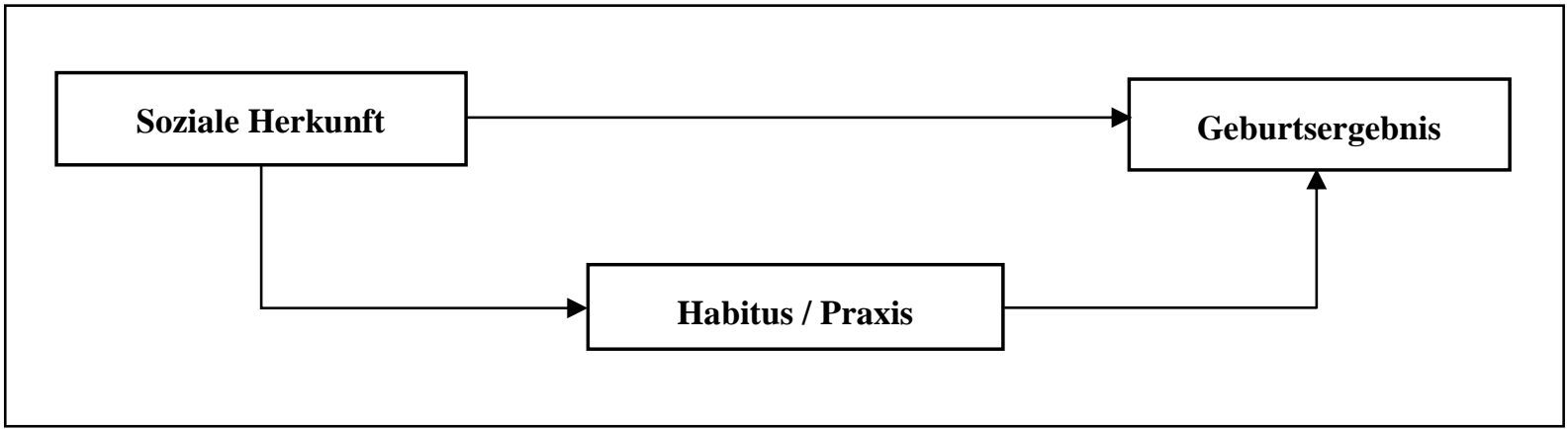

Anmerkung: Eigene Darstellung.

\subsection{Herkunftsbedingte Ungleichheiten im Vorsorgestatus Neugeborener}

Die Übernahme der Mutterrolle setzt mit Kenntnisnahme der Schwangerschaft ein und ist in der Regel mit Vollendung des ersten Lebensjahres des Kindes abgeschlossen (Barnard und Solchany 2002). Sie beinhaltet nicht nur eine liebevolle Fürsorge, sondern auch ein regelmäBiges gesundheitspräventives Engagement. Neben Armut und Stress gilt vor allem ein niedriger Bildungsgrad der (werdenden) Mutter als Risikofaktor für den Erwerb und die Bewältigung der Mutterrolle, wobei untere Bildungsschichten den alltäglichen Fürsorgeaufwand eher unterschätzen (Barnard und Solchany 2002).

Wie in der Einleitung dargelegt, weisen Kinder aus Familien, die unteren Bildungsschichten angehören, eher Präventionslücken auf. Aber auch Ein-Eltern-Familien und Migrantenfamilien nutzen die Us häufiger nicht in vollem Umfang (Kurz und Becker 2017; Lampert et al. 2005: 105). Bildungsproblematisch hieran ist die Besonderheit, dass im Mittel nun jene Us seltener besucht werden, welche lern- und schulrelevante Kompetenzen zum Gegenstand haben, ${ }^{9}$ weshalb bei ihnen die Kumulation anfänglicher Entwicklungsnachteile im Lebens- und Bildungsverlauf wahrscheinlicher ist.

\footnotetext{
${ }^{9}$ Die $U 7 a$ (34. bis 36. Lebensmonat) untersucht Motorik, Sprache, Hören, Sehen; die U8 (46. bis 48. Monat) zusätzlich dazu den Zahnzustand, Spiel- und Sozialverhalten und Ernährung sowie die U9 (60. bis 64. Monat) Beweglichkeit, Geschicklichkeit, Sprachentwicklung, Sozialverhalten, Interessen des Kindes und seine kognitive Entwicklung (Kamtsiuris et al. 2007; BVKJ). Seit 2006 haben die Bundesländer schrittweise ein verbindliches Einlade- und Meldewesen eingeführt, um die Selektivität der U-Teilnahme zu reduzieren (Stolberg 2017).
} 
In Westdeutschland ${ }^{10}$ gibt es für Kinder seit Anfang der 70er Jahre und seit 1991 ein für alle Bundesländer einheitliches mehrere Untersuchungen umfassendes Krankheitsfrüherkennungsprogramm (Baby: U1 bis U6; Kleinkind: U7 bis U9; J1 im Jugendalter, MeyerNürnberger 2002: 862). ${ }^{11}$ Der Besuch ist kostenlos (sofern Toleranzfristen nicht überschritten werden) und seit 2008/09 in den meisten Ländern und bei ausgewählten U-Untersuchungen auch Pflicht (Theiss et al. 2010). ${ }^{12}$ Erfolgen die ersten zwei Untersuchungen zumeist in der Geburtsklinik und erreichen nahezu alle Säuglinge, fällt ab der U5 (6. bis 7. Lebensmonat) bzw. der U6 (10. bis 12. Monat) mit zunehmendem Alter die Teilnahmerate je nach Herkunft unterschiedlich steil ab, besonders in weniger begünstigten Familien (Kurz und Becker 2017).

Vor diesem Hintergrund zielt die zweite Forschungsfrage darauf ab, soziologisch aufzuklären, wie es zu Bildungsdisparitäten im Vorsorgehandeln kommt. Aus sozialkapitaltheoretischer Hinsicht soll herausgefunden werden, ob die soziale Einbindung in familiäre Beziehungen soziale Ungleichheitsmuster in der Teilnahme an Vorsorgeuntersuchungen für Neugeborene (bis zum Alter von 18 Monaten) erklärt.

In diesem auf die sozialen Unterstützungsressourcen der Mütter abstellenden Zusammenhang interessieren eine Reihe Teilfragen: Fördert die soziale Integration - gemessen über das Vorhandensein einer Paarbeziehung - maßgeblich die Teilnahmebereitschaft? Wirken das Wohlbefinden mit der Mutterrolle und die Gesundheit der Mutter positiv auf das Teilnahmeverhalten? Lässt sich ein förderlicher Einfluss instrumenteller Unterstützung seitens des Partners auf die Inanspruchnahme der U-Untersuchungen erkennen? Welche Rolle spielen verwandtschaftliche Unterstützungsleistungen bei der Kindbetreuung für das Vorsorgehandeln? Im Hinblick auf die Mediation der Bildungsdifferenzen im Vorsorgestatus interessiert dabei hauptsächlich die Frage, ob die verschiedenen Sorten sozialen Beziehungskapitals sowie die gesundheitlichen Ressourcen der Mutter die Disparitäten in der Teilnahmebereitschaft vermitteln. Getestet wird die Annahme, dass soziale Unterschiede in der U-Teilnahme durch die unterschiedlich gute Einbindung in soziale Beziehungen vermittelt werden (Abbildung 3.2).

Das soziologische Forschungsfeld zur Thematik ist - wie die Literatur zur Gesundheitswirksamkeit sozialen Kapitals im Allgemeinen (Kroll und Lampert 2007: 122) - recht überschaubar. Inwiefern das Sozialkapitalkonzept zum Verständnis sozialer Unterschiede im elterlichen Präventionshandeln beiträgt, ist unzureichend erforscht. In der Literatur wird jedoch von einem Erklärungspotential des Sozialkapitalkonzepts für gesundheitliche Ungleichheitsfragen ausgegangen (Klocke 2004; Richter 2005; Poortinga 2006; Snelgrove et al. 2009).

\footnotetext{
${ }^{10}$ In der Deutschen Demokratischen Republik fanden Vorsorgeuntersuchungen in den Kindertagesstätten statt. Da fast alle Kinder institutionell betreut wurden, gab es somit fast keine soziale Besuchsselektivität (Kahl 2010).

${ }^{11}$ Inzwischen gibt es zwei neue U-Untersuchungen (U10: 7. Bis 8. Lebensjahr; U11: 9. bis 10. Lebensjahr), welche die große zeitliche Lücke zwischen der U9 (im fünften Lebensjahr) und der J1 (12. Bis 14. Lebensjahr) schließen sollen. Die Kosten dieser zusätzlichen Präventionsangebote werden im Unterschied zu den älteren Us nicht von allen Krankenkassen erstattet (BVKJ). Letzteres gilt auch für die neue J2 (Alter 16 bis 17).

12 Je nach U variiert der zeitliche Teilnahmespielraum. Die U6 kann zum Beispiel frühestens ab dem 9. und spätestens bis zum 14. Lebensmonat besucht werden. Danach tragen Eltern die Untersuchungskosten (BVKJ).
} 
Abbildung 3.2: Erklärungsmodell zu herkunftsspezifischem Präventionsverhalten

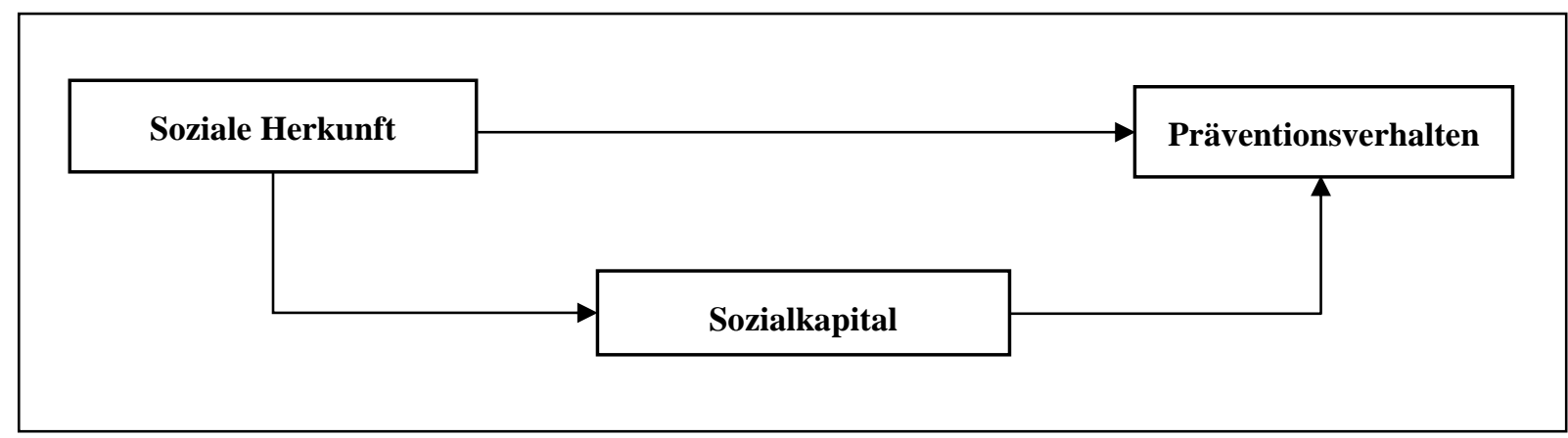

Anmerkung: Eigene Darstellung.

\subsection{Herkunftsbedingte Ungleichheiten im Bildungserfolg}

Hinterlässt ein nachteiliger Start ins Leben mittel- und langfristig Spuren beim Kompetenzund Bildungserwerb? Die Antwort auf diese bildungspolitische Frage lautet wahrscheinlich. Pessimistisch betrachtet legen Wechselwirkungen zwischen Säuglingsgesundheit und Bildung negative Effekte nahe (Lynch 2011: 523). Obwohl diese Frage in der deutschen Bildungsforschung gerade an Bedeutung gewinnt, wurde ihr aus den bereits angesprochenen Datenbeschränkungen - und womöglich auch aufgrund einer skeptischen Haltung der Soziologie gegenüber „entwicklungsbiologischen“ Einflüssen - kaum nachgegangen (Jäkel et al. 2013). Die Ursachen früher gesundheitlicher Ungleichheiten und ihrer Bildungskorrelate liegen deshalb noch im Halbdunkel und stellen wichtige soziologische Leerstellen in der Literatur dar. In welchen soziologischen Arbeiten und in welchen Kontexten wird auf die Schwangerschaft als Ursprung der sozialen Ungleichheitsgeschichte im Bildungswesen aufmerksam gemacht?

Die fetal-origin-These wurde im deutschen Forschungskontext im Zusammenhang mit den relativ stark ausgeprägten Bildungsnachteilen sozial benachteiligter (Wolke und Meyer 1999) Migrantenkinder (Kristen 2008) ins Feld geführt. Hintergrund hierfür ist, dass es insbesondere für Migrantenkinder bislang nur partiell gelungen ist ihren relativen Bildungsmisserfolg allein über sozioökonomische und sprachkulturelle Bedingungen aufzuklären (Esser 2006), was eine eine Unterspezifizierung der theoretischen Erklärungsmodelle nahelegt und Anlass zur Suche nach weiteren Ursachen gibt, die, wie bereits im Theoriekapitel herausgestellt, bei vorgeburtlichen Entwicklungs- und nachgeburtlichen Lebensphasen ansetzt (vgl. Kristen 2008). ${ }^{13}$

Ziel ist es nun herauszufinden, ob in untere Sozialschichten und Migrantenfamilien geborene Kinder möglicherweise deshalb in der Schule schlechter abschneiden, weil ihre Entwicklungschancen während der Schwangerschaft teilweise unter weniger guten Vorzeichen standen und ihre Beteiligung an den U-Untersuchungen zur Früherkennung gesundheitlicher und

\footnotetext{
${ }^{13}$ In Kapitel 2 wurde nicht gesondert auf die Rolle migrationsspezifischer (z.B. sprachkultureller Faktoren mit Blick auf die Verwendung und Beherrschung der Sprache des Ziellandes oder aspirationsbezogener Faktoren) eingegangen (hierzu der ausführliche Überblick bei Diehl et al. 2016). Auf einschlägige Literatur zu spezifisch ethnischen Faktoren, welche für den Erwerb von Gesundheit und Bildung bedeutsam sind, wird in der vorliegenden Arbeit jedoch an anderer Stelle (im Theorieteil von Kapitel 7) und dies detailliert mit Blick auf die hier im Untersuchungsfokus stehenden zwei Migrantengruppen bezuggenommen.
} 
kognitiver Entwicklungsnachteile im Mittel geringer ausfällt. Im Zentrum der Betrachtung stehen daher soziale und ethnische Ungleichheitserscheinungen in a) der Schulfähigkeit (Ergebnis der Einschulungsuntersuchung), b) den Grundschulnoten (Deutsch und Mathematik) sowie c) in der Sekundarschulpositionierung (Besuch eines weiterführenden Bildungsgangs).

Abbildung 3.3 veranschaulicht schematisch das Erklärungsmodell, in dem das Elternhaus als vor- wie nachgeburtlicher Risikofaktor für die Gesundheit Neugeborener und deren späteren Chancen im Bildungssystem konzeptualisiert wird (für eine differenziertere Darstellung siehe Kapitel 7). Die ausgewählten Bildungskriterien decken in ihrer Bandbreite verschiedene Erfolgsaspekte zu verschiedenen Zeitpunkten in der Bildungslaufbahn ab. Dies trägt dem geringen Kenntnisstand Rechnung, wie lang der Arm lebensanfänglicher Gesundheitsdisparitäten sowie präventionsbezogener Ungleichheiten tatsächlich reicht. Lassen sich „nur“ mittelfristige Effekte auf die Akkumulation von Kompetenzen bei Schulanfängern und Schulanfängerinnen feststellen? Oder gehen von schwangerschafts- und präventionsbezogenen Faktoren auch langfristige Effekte aus, etwa in Bezug auf Ungleichheiten im Notenerfolg in der Grundschule oder in der Bildungspositionierung? Für Deutschland ist dies eine offene Frage. Folglich ist es sinnvoll den Forschungszugang nicht a priori auf ein Bildungsmaß zu verengen.

Abbildung 3.3: Erklärungsmodell zu herkunftsspezifischem Bildungserfolg

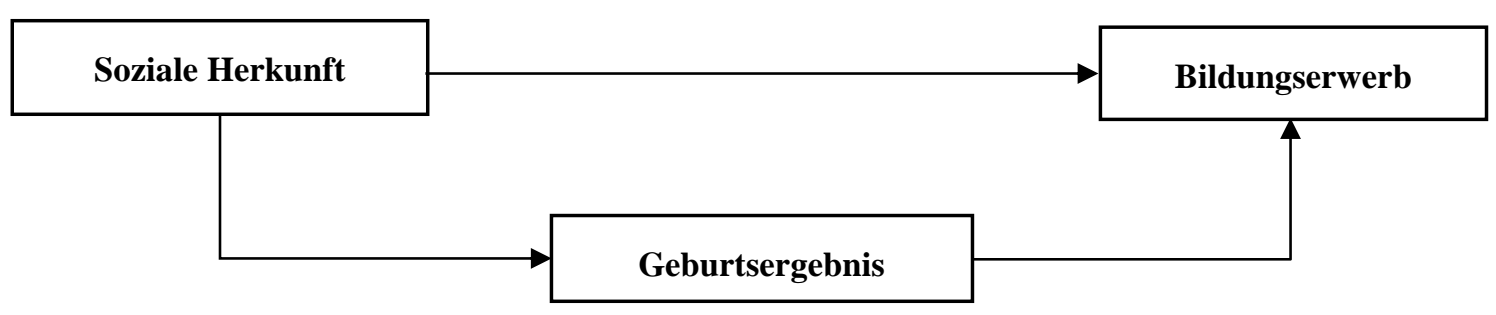

Anmerkung: Eigene Darstellung.

Im Mittelpunkt der Betrachtung stehen einerseits der Nachwuchs aus sozial weniger begünstigen Bevölkerungsschichten und andererseits der Nachwuchs aus Familien mit Migrationsgeschichte. Dabei wird der Migrationshintergrund möglichst differenziert erfasst, wobei zwischen zwei Herkunftsgruppen (und der Gruppe der Nicht-Migranten als Referenz) unterschieden wird: Kindern mit türkischen und ethnisch-deutschen Familienwurzeln. Beide Gruppen stellen in quantitativer Hinsicht einen bedeutsamen Anteil an der Bevölkerung Deutschlands. Der Fokus auf türkischstämmige Kinder wird zudem darüber motiviert, dass diese im deutschen Bildungssystem in der Vergangenheit (Alba et al. 1994) wie Gegenwart (Kristen und Dollmann 2009; Dollmann 2010) im Durchschnitt am schlechtesten abschneiden. Gleichzeitig fällt diese Gruppe durch eine relativ hohe Frühgeburtsprävalenz (RKI 2008) und hiermit assoziierter Anpassungsprobleme nach der Geburt auf (David und Pachaly 2010). Kinder mit ethnisch-deutschen Eltern (Spätaussiedlerfamilien) erzielen gegenüber türkischstämmigen Kindern relativ gute schulische Ergebnisse (Esser 2006; Fuchs und Sixt 2008; Gresch und Becker 
2010; Söhn 2008). Darüber hinaus startet diese Gruppe seltener mit gesundheitlichen Nachteilen ins Leben (Milewski und Peters 2014). Allerdings liegen zu Bildungserfolg und Säuglingsgesundheit von Spätaussiedlerkindern als Angehörige einer relativ jungen Zuwanderergruppe, welche in sozioökonomischer und sprachkultureller Hinsicht eine mittlere Position zwischen der türkischstämmigen Minderheits- und der deutschen Mehrheitsbevölkerung einnimmt (Strobel und Kristen 2015; Diehl et al. 2016), nur sehr wenige Forschungsarbeiten vor. Ihr Einbezug in die Theoriediskussion und in die Analysen dient daher als wichtige Kontrastfolie, um die ermittelten empirischen Befunde mit Blick auf ihre Plausibilität und Relevanz soziologisch besser einordnen zu können. 


\section{Datengrundlage und Methodik}

Um die im zweiten Kapitel skizzierten ressourcen- und lebenslauftheoretischen Überlegungen einer empirischen Bewährungsprobe zu unterziehen, bedarf es mit Blick auf die Erhebungsinhalte und den Stichprobenumfang anspruchsvollen Datenmaterials. Letzterer Aspekt ist insbesondere für die Untersuchung sozialgruppenspezifischer Strukturphänomene von Bedeutung.

Für die Beantwortung der Frage, ob Gesundheits- und Bildungschancen bereits vor der Geburt geprägt werden, sind differenzierte Informationen zu Schwangerschaft, Investitionsverhalten, Schulerfolg und Familienhintergrund notwendig. Die amtliche Datenlage zur Kindgesundheit ist recht dürftig (Kamtsiuris et al. 2007a) und hilft in diesem Kontext nicht weiter.

Um dennoch die theoretisch aufgezeigten Ungleichheitsmechanismen einem Test zu unterziehen, um in anderen Worten also „hinter die Fassaden gesellschaftlicher [Ungleichheits]Strukturen“ (Berger 1963: 42) schauen zu können, wird deshalb behelfsweise auf alternative sekundärstatistische Informationsquellen zurückgegriffen. Ein idealerweise den gesamten Lebenslauf abdeckendes, aktuelles und bundesweit repräsentatives Datengroßprojekt ist das Nationale Bildungspanel (The National Educational Panel Study: NEPS, siehe Blossfeld et al. 2011). Allerdings muss die NEPS-Neugeborenenkohorte erst noch „,altern“, um empirisch die Bedeutung schwangerschaftsbezogener Bildungsrisiken abschätzen zu können. Zum jetzigen Zeitpunkt geeignete Surveydaten stellen jedoch das Sozio-oekonomische Panel (SOEP) und der Kinder- und Jugendgesundheitssurvey (KiGGS) bereit. Im nachfolgenden Kapitel wird ein Kurzüberblick über beide Surveys gegeben. Über die Variablenkonstruktion und das methodische Vorgehen wird an anderer Stelle, nämlich in den Hauptkapiteln 5, 6 und 7 ausführlich Auskunft gegeben.

\subsection{Das Sozio-oekonomische Panel}

Für die Analysen bildungs- und gesundheitsbezogener Ungleichheitsfragen unter der Lebensverlaufsperspektive stellt das Sozio-oekonomische Panel (SOEP) seit 1984 als Personen- und Haushaltsbefragung bundesweit repräsentative Daten bereit (Wagner et al. 2007). Im Jahre 2003 schlug das Panel mit der Einführung des Zusatzfragebogens „Mutter und Kind“ eine interdisziplinäre Richtung ein.

Mit 19 Fragen zu verschiedensten Kennziffern zu Schwangerschaft, Neugeborenen- und Muttergesundheit, einschließlich der teils in separaten Personen- und Haushaltsdatensätzen erfassten ökonomischen, kulturellen und sozialen Ressourcenausstattung, bietet das SOEP Daten „von der Wiege bis zur Bahre“ (Lohmann et al. 2009: 252) zur Analyse entwicklungsbiologischer und gesundheitlicher (Ungleichheits-)Fragen. Perspektivisch werden in nicht all zu langer Zeit geeignete Daten für bildungsbezogene Verlaufsanalysen zur Verfügung stehen.

Die Hinwendung zu den frühesten Lebensphasen trägt der Einsicht Rechnung, dass Entwicklungs-, Gesundheits- und Bildungsprozesse einen vorgeburtlichen Ursprung haben. Um die gesundheitliche und kompetenzbezogene Entwicklung im Vorschulalter wie im Nachgang 
der Einschulung bildungswissenschaftlich begleiten zu können, wurden weitere Zusatzfragebögen zu verschiedenen Altersgruppen und Bildungsetappen implementiert. Zum Vorschulalter gibt es zwei weitere Mutter-Kind-Fragebögen (2. bis 3. sowie 5. bis 6. Lebensjahr). Für Analysen nach der Einschulung stehen Informationen zu (Grund)-Schulkindern im Alter von 7 bis 8 (im Einsatz seit 2010), 9 bis 10 (seit 2012) und 11 bis 12 (seit 2014) bereit (TNS 2015abc). Für das Jugendalter (Personen ab 17 Jahren) gibt es zwei Fragebögen. Ein Fragebogen erfasst seit dem Jahr 2000 Bildungsbeteiligung, Bildungserfolg (Noten), Bildungsaspirationen, berufliche wie familiäre Einstellungen und Freizeitaktivitäten von Jugendlichen. Mit dem zweiten seit 2006 eingesetzten Fragebogen werden kognitive Potenziale erhoben (Lohmann et al. 2009: 255). Neben diesem vielfältigen Erhebungshintergrund bezieht das SOEP sein Analysepotenzial für humankapitalbezogene Fragen zudem aus seinem prospektiven Erhebungsdesign, was es ermöglicht, Kausalitäten besser abzubilden (Lohmann et al. 2009).

\subsection{Der Kinder- und Jugendgesundheitssurvey}

Für eine Verbesserung der empirischen Datenlage zur Thematik sorgte unlängst der für Deutschland repräsentative Kinder- und Jugendgesundheitssurvey (KiGGS, Basiserhebung). Dieser enthält Ergebnisse von im Jahr 2006 beendeten Untersuchungen zum Gesundheitszustand von fast 18.000 Heranwachsenden (Scriba 2007: 531). Für die Untersuchung sozialer und ethnischer Bildungsunterschiede in Abhängigkeit schwangerschaftsbezogener sowie nachgeburtlicher Einflussgrößen (z. B. Eintrittsalter in die Kindertagesstätte) stellt der KiGGS eine einzigarte Informationsquelle dar (zum Surveykonzept: Kurth 2007; zum Stichprobendesign: Kamtsiuris et al. 2007b; zur Durchführung der Feldarbeit: Hölling et al. 2007). Im Unterschied zum SOEP - die Kinderpopulation (mit Migrationshintergrund) wird erst in einigen Jahren auf eine für Bildungsanalysen ausreichend große Fallzahl anwachsen - ermöglicht der KiGGS schon jetzt eine Untersuchung lebensanfänglicher Nachteile auf den Bildungserfolg nicht zuletzt dank der Stichprobengröße, die eine Differenzierung nach sozialen und ethnischen Herkunftsmerkmalen erlaubt (zur Messung soziodemographischer Merkmale, Lange et al. 2007; für die ethnische Herkunft, Schenk et al. 2007). ${ }^{14}$

Tabelle 4.1 stellt die Vor- und Nachteile beider Surveydatensätze und ihr Erhebungsprogramm vergleichend dar. Wie zu erkennen ist, eignen sich grundsätzlich beide Datenquellen für Analysen schwangerschaftsbezogener und gesundheitlicher Erscheinungen und dies auch spezifisch unter dem sozialen und ethnischen Herkunftsaspekt. Im Detail variieren aber die erhobenen Informationen, insbesondere mit Blick auf Informationen zur gesundheitlichen Lebensführung während der Schwangerschaft und medizinischer Indikationen für Schwanger-

\footnotetext{
${ }^{14}$ KiGGS wird aktuell als Panelstudie fortgeführt, wobei die Daten der Nachfolgeerhebung (KiGGS, Welle I, 2009-2012) seit August 2015 für wissenschaftliche Auswertungen genutzt werden können. Für die vorliegende Arbeit kam die Veröffentlichung dieser Daten jedoch zu spät. Anders als in der Basiserhebung, auf welcher die in Kapitel 6 vorgestellte Studie 4 basiert, stellt der Public-Use File der Nachfolgeerhebung keine Informationen zu Migrationshintergrund und Vorsorgeuntersuchungen bereit, sondern nur auf Anfrage im Rahmen eines Projektantrags (welcher Anfang Januar 2016 gestellt und abgelehnt wurde).
} 
schaftskomplikationen. So enthält das SOEP im Mutter-Kind-Fragebogen (0-1) weder Informationen zum Substanzkonsum (Tabak, Alkohol) noch zum Vorliegen etwaiger Komplikationen, wie zum Beispiel Schwangerschaftsdiabetes oder die Gewichtszunahme während der Schwangerschaft (als Indikator für Mangelernährung). Auch fehlen detaillierte Informationen zum Präventivhandeln nach der Schwangerschaft (die Teilnahme an den U-Untersuchungen ist nur am Beispiel der zuletzt besuchten U erhoben). Dafür stellt das SOEP aber Informationen zur Planung der Schwangerschaft, dem psychischen und physischen Wohlbefinden der (werdender) Mütter bereit, einschließlich ihres Zurechtkommens mit der Mutterrolle nach der Geburt und ihre soziale Einbindung und hierüber erschließbare emotionale und instrumentelle Unterstützungsressourcen mit der Kindesbetreuung und Erziehung im Alltag. Im Gegensatz dazu enthält die KiGGS-Basiserhebung differenziertere Informationen zu medizinischen und lebensstilbezogenen Schwangerschaftsrisiken, dafür aber keine Informationen zu Planung und gesundheitlichem Wohlbefinden während der Schwangerschaft. Auch fehlen Items zum sozialen Rückhalt und zur psychosozialen Bewältigung der Mutterrolle. Dafür ermöglicht KiGGS eine umfassendere Abbildung des Präventivhandelns im Rahmen der Vorsorgeuntersuchungen für Kinder. So wurde die Teilnahme für alle U-Untersuchungen einzeln abgefragt.

Tabelle 4.1: Vor- und Nachteile des Erhebungsprogramms und -designs von SOEP und KiGGS

\begin{tabular}{|c|c|c|}
\hline & SOEP (Mutter-Kind, 0-1) & KiGGS (Basiserhebung) \\
\hline Vorteile & $\begin{array}{l}\text { Prospektives Design; } \\
\text { Sozialer und ethnischer Familienhintergrund; } \\
\text { Einbindung in soziale Beziehungen; } \\
\text { Einstellungsebene (Habitus, Mutterrolle); }\end{array}$ & $\begin{array}{l}\text { Große Untersuchungspopulation; } \\
\text { Sozialer und ethnischer Familienhintergrund; } \\
\text { Erhebung von Lebensstil und medizinische } \\
\text { Risiken während der Schwangerschaft sowie } \\
\text { postnatales Präventivhandeln; }\end{array}$ \\
\hline Nachteile & $\begin{array}{l}\text { Junge Kohorte und relativ geringe } \\
\text { Stichprobengröße; } \\
\text { Keine Informationen zur Lebensführung wäh- } \\
\text { rend der Schwangerschaft; } \\
\text { Wenige Information zu medizinischen Schwan- } \\
\text { gerschaftsrisiken; }\end{array}$ & $\begin{array}{l}\text { Retrospektives Studiendesign; } \\
\text { Wenig(er) differenzierte Informationen zur } \\
\text { sozialen Einbindung; } \\
\text { Keine Informationen zu elterlichen Einstel- } \\
\text { lungen sowie Bewältigung der Mutterrolle; }\end{array}$ \\
\hline
\end{tabular}

Quelle: Eigene Darstellung

Zusammenfassend kann festgehalten werden: Vorteile der einen Datenbasis sind die Nachteile der anderen. Das gilt nicht nur hinsichtlich des Erhebungsprogramms, sondern auch mit Blick auf das Erhebungsdesign. Die Stärke des SOEP liegt in seinem Längsschnittcharakter, so dass die vorgeburtliche Ressourcenausstattung in Bezug gesetzt werden kann mit nachgeburtlich Kriterien zur Entwicklung und Gesundheit von Neugeborenen. Die Stärken des KiGGS beziehen sich auf die Vielfalt der Gesundheitsmerkmale (und der zusätzlichen Erhebung schulerfolgsrelevanter Informationen) sowie die relativ große Stichprobengröße (untersucht und ab dem elften Lebensjahr auch befragt wurden fast 18.000 Kinder und Jugendliche im Alter zwischen 0 bis 18 Jahren). Letzteres ist von besonderer Relevanz, um die Gesundheit und Bildung von Migrantenkindern untersuchen zu können. 


\subsection{Methodisches Vorgehen}

Zentrale Ziele der Arbeit sind die Beschreibung, insbesondere aber die Erklärung sozialer und ethnischer Differenzen in der Säuglingsgesundheit, im Investitionsverhalten und Schulerfolg.

Mit Blick auf die Ungleichheitsbeschreibung wird mithilfe einfacher Kreuztabellierungen und Korrelationsanalysen geprüft, ob und in welchem Ausmaß der soziale und ethnische Hintergrund Relevanz für das gesundheitliche und schulische Abschneiden der nachwachsenden Generation besitzt. Herauszufinden ist, ob wir es hinsichtlich dieser Humankapitaldimensionen mit ungleichheitssoziologischen Tatbeständen bzw. sozialen Fragen zu tun haben.

Mit Blick auf die Ungleichheitserklärung kommen in einem weiteren Schritt (multiple) regressionsanalytische Verfahren zum Einsatz. Diese ermöglichen die Untersuchung der vermuteten Kausalbeziehungen über den Einbezug von Drittvariablen (Kühnel und Krebs 2014: 482). Es wird angestrebt, regressionsanalytisch offenzulegen, welche Mechanismen hinter den zuvor deskriptiv aufgezeigten Ungleichheitsrelationen stehen. Hierfür wird in allen Analysen ein schrittweiser Aufbau der Regressionsmodelle gewählt. Beispielsweise gehen für die Untersuchung herkunftsspezifischer Bildungserfolgsunterschiede Variablenabbilder der zentralen sozialen und sozialepidemiologischen Erklärungsstränge zunächst getrennt und sodann erst in weiteren Spezifikationen gemeinsam in die Analysen ein.

In den nachfolgenden drei Kapiteln werden nun zu den drei Forschungsfragen die theoretische Herleitung der Hypothesen vorgenommen und die empirischen Analysen präsentiert. Den Bestimmungsgründen für soziale Ungleichheiten in Bezug auf die Wahrscheinlichkeit zu früh zur Welt zu kommen, widmet sich zunächst Kapitel 5 auf Basis der SOEP-Daten. Sozialen Ungleichheiten in den elterlichen Präventionsaktivitäten wird im Anschluss auf derselben Datengrundlage in Kapitel 6 auf den Grund gegangen. Auf Basis der Daten des Kinder- und Jugendgesundheitssurveys werden schließlich in Kapitel 7 die im Kontext mit einem aus entwicklungs- wie gesundheitsbezogener Sicht suboptimalen Frühstart ins Leben verbundenen und potentiell negativen Bildungsfolgen in den Untersuchungsfokus gerückt. 


\section{Gesundheitliche Ungleichheit zum Lebensbeginn}

\subsection{Frühchen - ein medizinisches und soziales Problem ${ }^{15}$}

$\mathrm{Zu}$ kurze Schwangerschaften (weniger als 37 Schwangerschaftswochen (SSW)) sind weltweit der häufigste Sterbegrund bei Neugeborenen (WHO 2005: 80 f. und 190 f.). Zudem verursachen die Langzeitfolgen der Frühgeburt neben den „kaum berechenbaren menschlichen Kosten" (Bergmann und Dudenhausen 2003: 391) hohe Ausgaben für das Gemeinwesen (ebd.; Petrou et al. 2001). So stützen zahlreiche Studien die in der Gesundheits- und Bildungsforschung prominente Kumulationshypothese, gemäß welcher anfängliche gesundheitliche Nachteile mit größeren Gesundheits- und Entwicklungsdefiziten im Lebenslauf oder geringem Schulerfolg einhergehen (für Gesundheit, siehe Rettwitz-Volk 2003: 143 ff.; MüllerRieckmann 2006: 17 ff.; Honein et al. 2009; für Bildung, siehe Bettge et al. 2014; Quigley et al. 2012; Wolke und Meyer 1999; Boyle und Boyle 2013; De Jong et al. 2012). Für die Konzeption geeigneter Präventionsmaßnahmen bedarf es somit empirisch fundierter Wissensbestände über die multiplen (Behrman und Butler 2007) Frühgeburtsdeterminanten (Blumenshine et al. 2010: 270).

$\mathrm{Zu}$ den vergleichsweise gut untersuchten medizinischen Risikofaktoren gehören Diabetes, genitale Infektionen, Fehlbildungen des Ungeborenen, Mehrlingsschwangerschaften, vorausgegangene Früh- und Todgeburten, Aborte und Schwangerschaftsabbrüche (Voigt et al. 2009; für einen Überblick über weitere medizinische Ursachen siehe Bühling und Friedmann 2009: 261). Zahlreiche Studien belegen zudem, dass junge und ältere Mütter sowie Erstgebärende und jene, die bereits zwei oder mehr Kinder geboren haben, höhere Frühgeburtenraten aufweisen (Khashan et al. 2010; Lumley 1993; Svensson et al. 2009; Straube et al. 2009; Bergmann und Dudenhausen 2003). Verhaltensindikatoren wie Tabak- und Alkoholkonsum, ungesundes Ernährungsverhalten, sportliche Betätigung und die geringe Inanspruchnahme der Schwangerenvorsorge gelten ebenfalls als bedeutsame Prädiktoren. Darüber hinaus steht die weibliche Konstitution vor der Schwangerschaft in einem umgekehrt linearen (Körpergröße) oder u-förmigen (Körpergewicht, Body-Mass-Index) Zusammenhang (Voigt et al. 2006a, 2006b; vgl. Abschn. 2.3). Die schwangerschaftsverkürzende Wirkung psychosozialer Merkmale wie Stress, kritische Lebensereignisse, Geburtsangst, ungewollte Schwangerschaft, Depression und fehlende soziale Unterstützung ist hingegen nicht abschließend geklärt (Behrman und Butler 2007: 104 f.). Zu den einflussreichsten sozioökonomischen Merkmalen gehören schließlich die berufliche Stellung, das Einkommen (Reime et al. 2006; Straube et al. 2009; Tamm 2005; Peacocket al. 1995), die mütterliche Bildungsnähe (für Deutschland siehe

\footnotetext{
${ }^{15}$ Der in diesem Kapitel enthaltene Beitrag entstand in Zusammenarbeit mit Carolyn Stolberg und erschien im Jahr 2015 in der Kölner Zeitschrift für Soziologie und Sozialpsychologie unter dem Titel „Gesundheitliche Ungleichheit zum Lebensbeginn - Zum Einfluss der mütterlichen Bildung auf die Wahrscheinlichkeit einer Frühgeburt"(Stolberg und Becker 2015). Die Redaktion gab den Wiederabdruck frei.
} 
Peters 2010; Kemptner und Marcus 2012; für internationale Evidenz siehe Ancél et al. 1999a,b; Currie und Moretti 2003) und, bislang kaum untersucht, der Bildungsgrad des Partners (Blumenshine et al. 2011; Misra et al. 2010; Becker und Stolberg 2013).

Wie für Morbiditäts- und Mortalitätsunterschiede im Allgemeinen (vgl. Jungbauer-Gans 2006: 87) bestehen auch für die soziologische Analyse des sozialen Gradienten in der Schwangerschaftsdauer noch große Erklärungsdefizite (Behrman und Butler 2007). Während die Selektionshypothese gesundheitliche Ungleichheit als Folge „einer sozialen Aufstiegsbewegung der Gesunden“" annimmt (Richter und Hurrelmann 2009: 20; Hervorhebung im Original) und soziale Mechanismen ausblendet, spricht die empirische Evidenz überwiegend für die (hier im Fokus stehende) Verursachungshypothese, welche sich bei der Erklärung sozialer Gesundheitsunterschiede auf materielle, kulturell-verhaltensbezogene und psychosoziale Argumentationslinien stützt (Richter und Hurrelmann 2009: 21; Jungbauer-Gans und Kriwy 2004: 14; Richter 2005: 139).

Für das Verständnis der Frühgeburt als multikausales Phänomen eignet sich die Kapitaltheorie von Pierre Bourdieu (1983) im besonderen Maße, denn neben dem Vorteil einer starken empirischen Basis (Brake et al. 2013) bietet sie die Möglichkeit, Verhältnis- und Verhaltensebene miteinander zu verknüpfen (Rössel 2009: 310). Dem kulturellen Kapital kommt aufgrund der engen Wechselbeziehung zum Habitus (gesundheitsbezogene Wahrnehmungs-, Denk- und Handlungsschemata) eine übergeordnete Rolle zu (Abel 2007, 2008).

Diesen theoretischen Anker als Ausgangspunkt nehmend, steht der sozial ungleiche „Frühstart ins Leben“ (Bernthaler 2009) im Mittelpunkt des vorliegenden Beitrags. Genauer gilt es folgende Fragestellung theoretisch zu diskutieren und empirisch zu beantworten: Vermitteln ökonomische und soziale Ressourcen sowie riskante Einstellungen und Verhaltensweisen das bildungsspezifische Frühgeburtsrisiko?

Für Deutschland liegen kaum Untersuchungen zur Erklärung der sozialen Frühgeburtsproblematik vor. Zwei auf dem sozio-oekonomischen Panel (SOEP, Mutter-Kind-Fragebogen) basierende Studien (Peters 2010; Kemptner und Marcus 2012) nehmen dieses Phänomen jedoch in den Blick. Während Peters das Wechselspiel zwischen mütterlicher Bildung und Lebensalter prüft, interessieren sich Kemptner und Marcus (2012) für den Kausaleffekt der mütterlichen Bildung auf die Schwangerschaftsdauer. Zentraler Befund beider Untersuchungen ist der Fortbestand bildungsspezifischer Frühgeburtsrisiken, selbst nach statistischer Kontrolle eines umfangreicheren (vgl. Peters 2010) oder sparsameren (vgl. Kemptner und Marcus 2012) Drittvariablenbündels. ${ }^{16}$ Ein anders gelagertes Erklärungsziel verfolgend, lenken wir die Aufmerksamkeit nicht nur auf kulturelle und ökonomische Einflüsse oder das Gesundheitshandeln (hier: Tabakkonsum, Body-Mass-Index, sportliche Betätigung, Teilnahme an der Schwangerenvorsorge), sondern berücksichtigen auch mit dem Habituskonzept verknüpfte Argumente (persönliche Risikobereitschaft und Kontrollüberzeugungen). Ferner fragen wir unter einer sozialkapitaltheoretischen Perspektive nach der Gesundheitswirksamkeit sozialer

\footnotetext{
${ }^{16}$ Die Verhaltensebene wird von Peters (2010), nicht aber von Kemptner und Marcus (2012) berücksichtigt.
} 
Beziehungen (vgl. Becker und Stolberg 2013). Denn „nicht alle Individuen [darunter zunehmend niedrig gebildete Frauen]“ (Lengerer 2012: 271) leben in einer Partnerschaft. Dies kann sich negativ auf die Wahrnehmung präventiver Gesundheitsangebote und den Schwangerschaftsverlauf auswirken (Spallek et al. 2014). Ebenfalls wird untersucht, inwiefern regelmäBige Kontakte zu Personen aus dem Verwandtschafts-, Nachbarschafts- und Freundeskreis relevant für das Verständnis bildungsabhängiger Frühgeburtsrisiken sind.

Im Folgenden stellen wir unser theoretisches Modell zur Erklärung sozialer Frühgeburtsdisparitäten vor (Abschn. 2). Das methodische Vorgehen wird in Abschn. 3 beschrieben. Darauf folgt die Darstellung der Ergebnisse (Abschn. 4) und abschließend deren Zusammenfassung und Diskussion (Abschn. 5).

\subsection{Bourdieus Kapitaltheorie zur Erklärung gesundheitlicher Ungleichheit zum Le- bensbeginn}

Unsere theoretischen Überlegungen skizzieren wir entlang der in Abbildung 5.1 schematisch dargestellten Erklärungskette Struktur - Habitus/Praxis - Gesundheit. Gesundheitsdisparitäten haben demnach einen sozialen Ursprung (Mielck 2005). Deshalb erwarten wir grundsätzlich potenziell bedeutsame Effekte aller drei Kapitalformen, wobei das jeweilige Einflussgewicht variieren sollte (Abel et al. 2009). Wir nehmen an, dass die Schwangerschaftsdauer in erster Linie vom kulturellen Kapitalvolumen (formale Bildung) abhängt (Abschn. 3.2.1), während die Wirkung ökonomischen (Abschn. 3.2.2) und sozialen Kapitals (Abschn. 3.2.3) schwächer ausgeprägt ist. Genauer sollte der Zusammenhang zwischen mütterlicher Bildung und Schwangerschaftsdauer durch die anderen zwei Kapitalformen vermittelt (Mediation) und in seiner Stärke moderiert werden. ${ }^{17}$ Wie im Erklärungsmodell ersichtlich, sind Bildungsunterschiede in der Schwangerschaftsdauer nicht ausschließlich das Ergebnis einer geringen Ressourcenausstattung, sondern auch Ausdruck einer über den Pfad der Nabelschnur laufenden (vgl. Bergmann und Dudenhausen 2003: 392) hochgradig verschleierten Gesundheitspraxis, welche durch übergreifende, eng mit dem Kulturkapital verwobene, Einstellungen (Habitus) geprägt ist (Abschn. 3.2.4). ${ }^{18}$

\footnotetext{
${ }^{17}$ Eine ausführliche Theoriediskussion möglicher Interdependenzen zwischen kulturellem und ökonomischem oder sozialem Kapital wird an dieser Stelle nicht geleistet, da uns deren empirische Überprüfung aus methodischen Gründen (geringe Zellbesetzung in den Interaktionskategorien aufgrund des seltenen Ereignisses einer Frühgeburt) nicht sinnvoll erscheint. Der Vollständigkeit halber skizzieren wir dieses Wechselspiel in der Schlussbetrachtung (Abschn. 5).

${ }^{18}$ Dem Zusammenspiel aller Ursachenkomplexe tragen wir durch Querverbindungen in den Unterabschnitten Rechnung.
} 
Abbildung 5.1: Die Kapitaltheorie zur Erklärung gesundheitlicher Ungleichheit

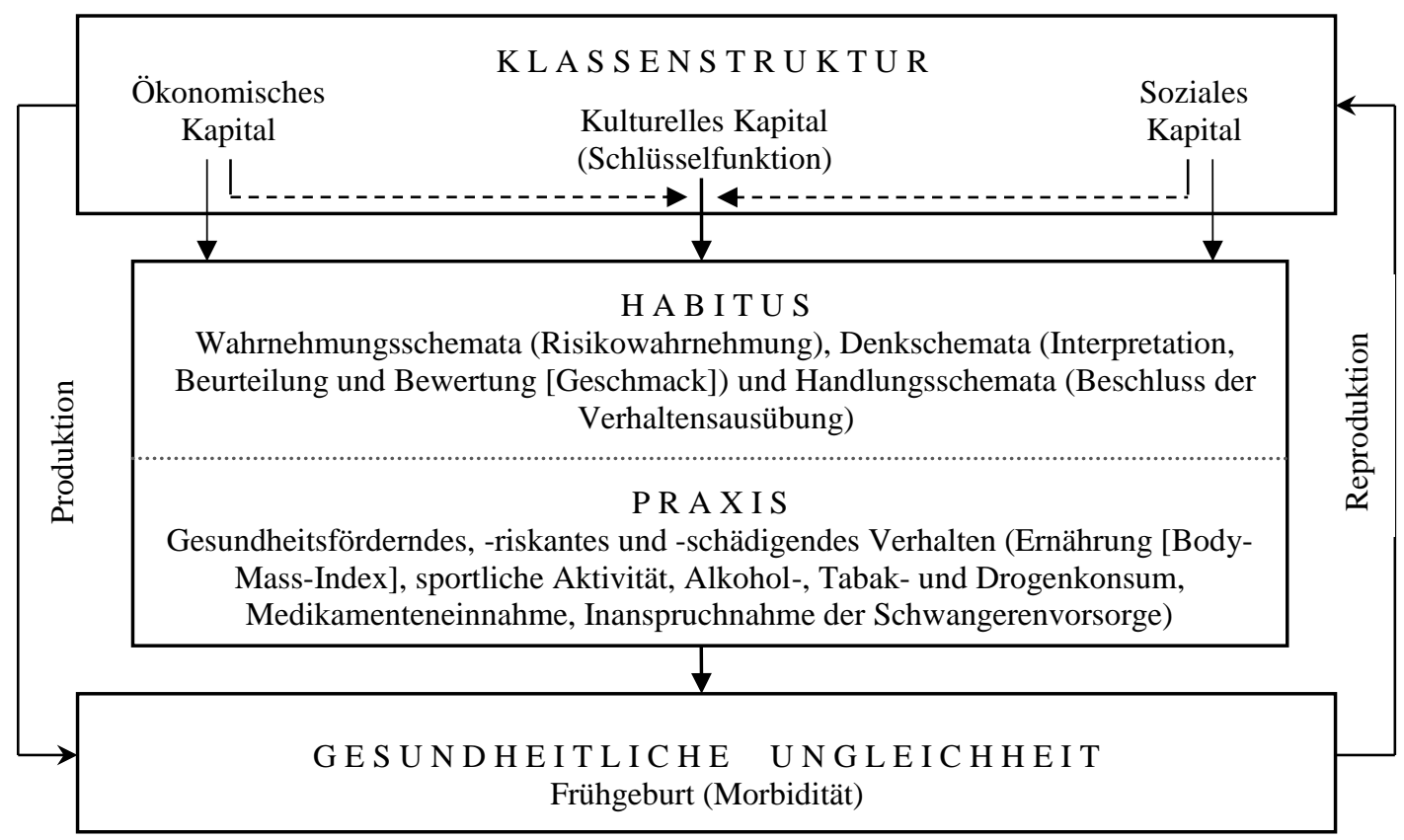

Quelle: Eigene Darstellung (in Anlehnung an Abel et al. 2009: 203, Abel 2008: 2, Mielck 2005)

Anmerkung: Gestrichelte Linien verdeutlichen moderierende Einflüsse.

\subsubsection{Das Kulturkapital als zentraler Frühgeburtsprädiktor}

Wie kulturelles Kapital im Allgemeinen (Bourdieu 1983: 187 ff.), hat auch gesundheitskulturelles Kapital drei Erscheinungsformen (vgl. Abel et al. 2009: 199 ff.). Im Hinblick auf die Schwangerschaft zählen themenbezogene Publikationen und Broschüren wie „Rundum Schwangerschaft und Geburt“ (BZgA 2014) oder „Ich bin dabei! - Vater werden“ (BZgA 2012) zu gesundheitsbezogenem Kulturkapital in objektivierter Form. Diese informieren über die hohe Bedeutung eines gesundheitsförderlichen Verhaltens während der Schwangerschaft (z. B. Rauch- und Alkoholverzicht, regelmäßige Schwangerenvorsorge, ausgewogene Ernährung) und klären über gesetzliche Ansprüche und Pflichten auf (bspw. das Recht auf Hebammenhilfe nach § 24d SGB V, das Recht auf Beratung während der Schwangerschaft nach § 16 Abs. 3 SGB VIII oder die Mitteilungspflicht an den Arbeitgeber nach $\S 5$ Abs. 1 MuSchG). Geld zur materiellen Aneignung (Bourdieu 1983: 189) setzen diese Kulturgüter nicht zwangsläufig voraus. ${ }^{19}$ Allerdings ist für deren „Genuß“ eine angemessene Nutzung und Beherrschung von inkorporiertem Kulturkapital (symbolische Erschließung) erforderlich und notwendig (Bourdieu 1983: 189). ${ }^{20}$ Inkorporierte Wissensbestände und Fähigkeiten darüber, „wie Gesundheit zustande kommt [,]...wie sie verbessert [oder erhalten] werden kann“, sind

\footnotetext{
${ }^{19}$ Der Zugang zu Informationsträgern (Bücher, Online-Broschüren) kann kostenpflichtig sein, zum Beispiel im Rahmen einer Bibliotheksmitgliedschaft oder bei Anschaffung und Betrieb eines internetfähigen technischen Gerätes. Analog können Kosten für den Kauf oder die Nutzung weiterer gesundheitsbezogener Kulturgüter anfallen (vgl. Abel et al. 2009).

${ }^{20}$ Die symbolische Erschließung setzt eine Informationssuche voraus (vgl. Abel et al. 2009: 198), deren Initiierung und Erfolg vom gesundheitsbezogenen Vorwissen und gesundheitlichen Einstellungen abhängen.
} 
im Alltag besonders gesundheitsrelevant, da sie sich in bestimmten Einstellungen und Lebensstilen niederschlagen (Abel et al. 2009: 200 f.).

Zur Abbildung inkorporierter Wissensbestände und Kompetenzen dient in empirischen Studien überwiegend das institutionalisierte kulturelle Kapital. Dies liegt darin begründet, dass kaum Daten zum verinnerlichten Gesundheitskapital vorliegen. Die formale Bildungsqualifikation stellt somit, wie in der empirischen Bildungsforschung, den ,wichtigste[n] Indikator ...[dar]“ (Kratzmann und Schneider 2009: 6). Obwohl Bildungstiteln ,keine reale Garantie [über das verinnerlichte Wissen] abzuverlangen ist ..." (Bourdieu 1987: 51; vgl. Bourdieu 1983: 190; Abel et al. 2009: 201), spiegeln diese zum Teil das in formellen Lernkontexten erworbene Gesundheitswissen wider (für schulformabhängige Ernährungsgewohnheiten und -wissen: vgl. BZgA 2003; Gerhards et al. 2004), welches zur aktiven und effizienten Förderung der Gesundheit befähigen kann (vgl. Currie und Moretti 2003). Neben der Qualifikationsfunktion zielen Bildungsinstitutionen auch auf die Aneignung einer oberen Bildungsschichten vertrauten distinguierten (eher gesundheitszuträglichen) „Lebensführung“ (Weber 1972: 587, zit. nach Bremer 2006: 187). Frauen mit höherer Bildungsqualifikation dürften also bereits vor der Schwangerschaft über ein umfangreicheres Wissen zur Herstellung und Erhaltung von Gesundheit verfügen. Hierzu zählt auch eine größere Vertrautheit mit dem Gesundheitssystem (z. B. bezüglich Existenz und Ablauf der Schwangerenvorsorgeuntersuchungen). Darüber hinaus können höher Gebildete Gesundheit im Alltag effizienter herstellen, weil sie neben einem umfangreicheren Gesundheitswissen auch über eine, durch schulische Lernprozesse vermittelt, größere Kontrolle über ihr eigenes Leben verfügen (Mirowsky und Ross 2007: 1342; 2003).

Zusammenfassend lässt sich für das kulturelle Kapital als zentraler Frühgeburtsprädiktor folgende, empirisch gut bestätigte (zusammenfassend Xie et al. 2014; vgl. auch van den Berg 2012; für Deutschland siehe Peters 2010; Kemptner und Marcus 2012) Hypothese aufstellen:

H1: Frauen mit der niedrigsten Bildungsqualifikation (maximal Hauptschulabschluss ${ }^{21}$ ) haben im Vergleich zu Frauen mit weiterführender Bildung ein erhöhtes Frühgeburtsrisiko.

\subsubsection{Direkte und vermittelnde Effekte ökonomischen Kapitals}

Die gesundheitliche Bedeutung ökonomischen Kapitals, jedwedes in Geld umwandelbares Vermögen (Bourdieu 1983: 185; Bourdieu 1987: 199; Abel et al. 2009: 200), besteht zuerst in der Existenzsicherung (Mirowsky und Ross 2003: 194). Zwar gilt in modernen Gesellschaften die Befriedigung menschlicher Grundbedürfnisse als weithin gegeben (Blumenshine et al. 2010: 269; Mirowsky und Ross 2003: 194), doch hängen ökonomische Privilegien, wie geringere Schadstoff- und Lärmbelastungen im Wohnumfeld (Currie und Moretti 2003), bessere Wohnbedingungen (Unger 2008) und niedrigere Zugangsbarrieren zum Gesundheitssystem (Mielck 2005) typischerweise mit geringeren Frühgeburtsanteilen zusammen (Blumenshine et

\footnotetext{
${ }^{21}$ Es ist zu beachten, dass der Hauptschulabschluss nicht zwingend in einer Hauptschule, sondern auch in alternativen Schulformen erworben werden kann. Dies gilt analog für den Realschulabschluss.
} 
al. 2010). Zudem sind ökonomisch Bessergestellte, selbst bei gleicher Exposition zu Umweltbelastungen und gleichen gesundheitlichen Versorgungsopportunitäten, im Alltag nicht nur finanziell (Currie und Moretti 2003), sondern, sofern Einkommensarmut psychosoziale Belastungen hervorruft (Collatz et al. 1979), auch psychisch eher in der Lage, gesundheitsorientiert zu handeln. Darüber hinaus hat ökonomisches Kapital für soziale und kulturelle Teilhabe(bedürfnisse) Relevanz (Bourdieu 1987). Aus diesen Gründen ist anzunehmen:

H2a: Einkommensarmut geht mit einem erhöhten Frühgeburtsrisiko einher.

Da eine Abhängigkeit der Gesundheit von ökonomischen Kapitalien im untersten Bildungssegment am größten ist (Mirowsky und Ross 2003: 194), ist weiterhin anzunehmen:

H2b: Erhöhte Frühgeburtsrisiken lassen sich bei formal niedrig gebildeten Frauen teilweise auf eine schlechtere finanzielle Lage zurückführen.

Eine vollständige Aufklärung des Zusammenhangs zwischen Bildung und Frühgeburtsstatus ist allerdings unwahrscheinlich, denn die Wahl gesundheitsbezogener Praxen ist vielmehr von kulturellen Ressourcen und damit verknüpften Einstellungen (Abel 2008: 3; Klein et al. 2001: 385) abhängig als von finanziellen Zwängen (Blumenshine et al. 2010: 269; Mirowsky und Ross 2003: 194; für Ernährungsverhalten, Gerhards et al. 2004: 157). Zudem stützen empirische Befunde die Vorrangstellung der Bildung (für einen aktuellen Forschungsüberblick siehe Blumenshine et al. 2010): Während das Kulturkapital konsistent in einem linearen $\mathrm{Zu}$ sammenhang mit der Schwangerschaftsdauer steht, zeigt sich für das Einkommen teils (nur) ein „threshold effect“ (ebd.: 267). Ökonomische Erklärungen geben also in der Regel keine befriedigende Antwort auf die Frage, warum einkommensarme Bevölkerungsgruppen verstärkt Tabak konsumieren, sich schlechter ernähren (Gerhards et al. 2004: 157), seltener sportlich betätigen oder unregelmäßiger an Vorsorgeuntersuchungen teilnehmen, obwohl die Ausgaben für Genussmittel hoch sind und ungesundes Essen nicht immer billiger ist (Feinstein 1993: 306 f.), Sportaktivitäten nicht zwangsläufig materielle Kosten verursachen und (Standard-)Vorsorgeuntersuchungen in der Regel kostenfrei sind (Hurrelmann 2006).

\subsubsection{Direkte und vermittelnde Effekte sozialen Kapitals}

Sozialkapital, alle „Ressourcen, die auf der Zugehörigkeit zu einer Gruppe beruhen...“ (Bourdieu 1983: 191, Hervorhebung im Original), kann im Wesentlichen in die Beziehungsquantität (soziale Integration) und Beziehungsqualität (soziale Unterstützung, soziale Regulation/Kontrolle, soziale Konflikte) zerlegt werden (House et al. 1988). Empirische Studien verweisen auf gesundheitliche Nachteile bei Personen mit keinerlei oder flüchtigen sozialen Beziehungen (D'Hombres et al. 2010; Kuper et al. 2002; Pevalin und Rose 2002; Weyers 2008: 263 f.). Mit Blick auf die Schwangerschaft gilt, dass Alleinstehende gegenüber in Partnerschaften lebenden Frauen höhere Frühgeburtsrisiken haben (Luo et al. 2004; siehe Shah et al. 2011 für einen Überblick). Frauen mit niedriger formaler Bildung sind in der Regel seltener in eine Partnerschaft integriert (Lengerer 2012). Wichtige Ressourcen, von denen Schwangere gesundheitlich profitieren könnten, bleiben damit aus. Neben einer zunehmenden 
Notwendigkeit eines Partnereinkommens (Dingeldey und Berninger 2013) zur finanziellen Deckung des Haushaltsbedarfs bietet das Zusammenleben mit einem Partner auch emotionalen Rückhalt, welcher die in kritischen Lebensphasen (wie der Schwangerschaft) oder bei Einkommensarmut (Lampert und Ziese 2005: 48) potenziell erhöhten Belastungen auffangen kann. Allein das Wissen um zugängliche Unterstützungsquellen führt in Stresssituationen zu einem „Puffereffekt“ (Jungbauer-Gans 2002: 57; vgl. Heaney und Israel 2008: 193 f.; Klauer 2009: 80 ff.), was wiederum einem Risikoverhalten in der Schwangerschaft (z. B. Rauchen) vorbeugt (Sperlich et al. 2011).

Ferner können soziale Kontroll- und Regulierungsprozesse das Gesundheitsverhalten sowohl positiv als auch negativ beeinflussen (House et al. 1988: $302 \mathrm{ff}$.). Im positiven Sinne wirkt das Vorhandensein eines Partners als Verhaltenskorrektiv (Misra et al. 2010). ${ }^{22}$ So nehmen in Partnerschaft lebende Frauen im Vergleich zu Alleinstehenden früher und regelmäßiger Schwangerenvorsorgeuntersuchungen in Anspruch (Misra et al. 2010; Teitler 2001; Spallek et al. 2014). Vor dem Hintergrund dieser sozialkapitaltheoretischen Überlegungen ergeben sich folgende Hypothesen:

H3a: Für in Partnerschaft lebende Frauen sind geringere Frühgeburtsrisiken zu erwarten. Weil auch Familienangehörige, Freunde oder Nachbarn Unterstützungsquellen darstellen und potenziell verhaltensregulierend wirken, gilt diese Annahme ebenso für regelmäßige Besuche anderweitiger Bekanntschaften.

H3b: Für in Partnerschaft lebende Frauen ist ein über soziale Kontroll- und Regulierungsprozesse vermittelter Einfluss der formalen Bildung des Partners auf das Frühgeburtsrisiko zu erwarten.

Mit Blick auf Hypothese H3b sollte das Risiko mit steigendem Bildungskapital abnehmen (vgl. Shah et al. 2011). Denn aufgrund bildungshomophiler Partnerwahl (Weyers 2008: 260; Skopek et al. 2009) ähneln sich soziale Netzwerke hinsichtlich ihres gesundheitskulturellen und ökonomischen Kapitals (Bourdieu 1987; Williams 1995). Deshalb kann für niedrig gebildete Frauen die Wahrscheinlichkeit negativer Sozialkapitaleffekte (z. B. Ausbleiben der Verhaltenskorrektur, Exposition zu Passivrauch) höher ausfallen. ${ }^{23}$

H3c: Die Gesundheitswirksamkeit der Sozialkapitalindikatoren ${ }^{24}$, einschließlich bedeutsamer Assoziationen zur mütterlichen Bildung vorausgesetzt, dürften die Bildungsunterschiede im Schwangerschaftsergebnis zurückgehen.

\footnotetext{
${ }^{22}$ Neben sozioökonomischen (Bildung, berufliche Stellung) und psychosozialen Einflüssen spielt der Partner auch unter biomedizinischen Aspekten (z. B. Alter, Körpergröße und -gewicht, eigenes Geburtsgewicht) eine bedeutsame Rolle für die Dauer der Schwangerschaft (Shah 2010).

${ }^{23}$ Ferner können soziale Beziehungen auch als Belastung angesehen werden (soziale Konflikte), insbesondere dann, wenn diese „,aufgrund institutioneller Regelungen oder existentieller Notwendigkeit nicht der Wahlfreiheit unterliegen“" (Jungbauer-Gans 2002: 59).

${ }^{24}$ Anhand des Bildungsniveaus des Partners ist es möglich, nicht nur die Quantität sozialen Kapitals (siehe hierzu Kroll und Lampert 2007), sondern (näherungsweise) auch die Qualität abzugreifen (vgl. Becker und Stolberg 2013).
} 
Allerdings ist auch eine vollständige Aufklärung des Bildungsgradienten durch Sozialkapital unwahrscheinlich, da vorliegende empirische Evidenzen (Shah et al. 2011) zwar auf signifikante, aber im Vergleich zum kulturellen Kapital nur schwache Assoziationen mit dem Frühgeburtsrisiko verweisen.

\subsubsection{Habitus und Lebensstil als Bindeglieder zwischen Sozialstruktur und Gesundheit}

Wie eingangs erwähnt, stehen insbesondere Kulturkapital und Lebensführung in einem Spannungsverhältnis, welches durch eine grundlegende Orientierung, dem Habitus, reguliert wird. Genauer handelt es sich hier um verinnerlichte Dispositionen (Wahrnehmungs-, Denk- und Handlungsschemata), welche aufgrund gesellschaftlicher Zwänge nicht überschreitbaren Grenzen unterliegen (Bourdieu 1987).

Für die gesundheitsspezifische Orientierung ist anzunehmen, dass sich Wahrnehmungen und Überzeugungen (,health beliefs"), die „...Menschen von Gesundheitsrisiken haben (allgemeine Risikowahrnehmung) und wie sie diesbezüglich ihre eigene gesundheitliche Gefährdung einschätzen (selbstbezogene Risikowahrnehmung)“ (Faltermaier 1994: 75, Hervorhebung im Original; vgl. Abel et al. 2009: 198), je nach Bildungsstatus unterscheiden. Bezogen auf die Schwangerschaft ist bei einer geringen Ausprägung des Kulturkapitals (inkorporierte Wissensbestände über Gesundheitsrisiken) nun möglich, dass Komplikationen nicht unmittelbar in einen Zusammenhang mit Alkoholkonsum gebracht werden oder der Gang zur Schwangerenvorsorge für deren Abwendung nicht unbedingt notwendig erscheint (allgemeine Risikowahrnehmung). Zudem könnten untere Bildungsgruppen eher zu einer Unterschätzung der Wirkung gesundheitsschädigender Verhaltensweisen (diese eine Zigarette hat keinen negativen Auswirkungen auf meine Schwangerschaft) neigen (selbstbezogene Risikowahrnehmung). ${ }^{25}$

\section{H4a: Aufgrund der engen Verbindung zwischen Bildung und Risikowahrnehmung dürften gesundheitszuträgliche Wahrnehmungsschemata (hier: geringe Risikobereitschaft) so- mit bei jenen Frauen am stärksten ausgeprägt sein, die über ein höheres Kulturkapital verfügen.}

Denkschemata beinhalten die Interpretation, Beurteilung und Bewertung (Geschmack) von genau diesen gesundheitsrelevanten Wahrnehmungen. Der Geschmack markiert die Klassengrenzen, indem dieser ,... bewirkt, daß man hat, was man mag, weil man mag, was man hat, nämlich die Eigenschaften und Merkmale, die einem de facto zugeteilt und durch Klassifikation de jure zugewiesen werden“ (Bourdieu 1987: 286). So ermöglicht der die oberen Klassen definierende Luxusgeschmack Wahlfreiheit, gibt zugleich den Geschmack der mittleren Klas-

\footnotetext{
${ }^{25}$ Die Risikobereitschaft zu gesundheitsschädigendem Verhalten hängt auch vom Grad materieller Deprivation und seinem Bezug zu Empfinden und Umgang mit Stress ab. So verweisen Lampert und Ziese (2005: 34 f.) darauf, dass ,[b]ei der Befriedigung individueller Bedürfnisse eine kurzfristige Orientierung [überwiegt], aus der eine höhere Bereitschaft zu gesundheitsriskantem Verhalten resultiert, insbesondere wenn dieses wie beim Rauchen oder Alkoholkonsum unmittelbar zur Stressbewältigung beiträgt. Auch ungesunde Ernährungsweisen und Bewegungsmangel können als Verhaltensreaktionen auf eine hohe Stressbelastung verstanden werden“.
} 
sen vor und legt den durch Wahlzwang gekennzeichneten und „durch Lebensumstände geschaffen[en]“ Notwendigkeitsgeschmack der unteren Klassen fest (Bourdieu 1987: 289 ff.; vgl. Beckert-Ziegelschmid 2005: 32; Williams 1995: 589 f.).

Denkschemata beinhalten demnach Kontrollüberzeugungen im Hinblick auf die „Veränderbarkeit" (Abel et al. 2009: 198) bestimmter Lebensumstände und Verhaltensweisen sowie Überzeugungen, über das eigene Leben eigenständig verfügen zu können. Wie bei den Wahrnehmungsschemata, wird auch bei den Denkschemata dem kulturellem Kapital eine besonders hohe Bedeutung beigemessen (Abel et al. 2009: 200). So haben im Elternhaus mitgegebene und im Bildungssystem ausgeformte positive Kontrollüberzeugungen (vgl. hierzu auch Mirowsky und Ross 2007) die Funktion, ein bestimmtes Gesundheitsverhalten zu verinnerlichen. Bei formal gering gebildeten Frauen resultiert aus der „Kombination von geringer Kontrollüberzeugung, und einem instrumentellen Gesundheitsverständnis“" eine aus gesundheitlicher Sicht negativ geprägte Grundeinstellung, „[welche]...sich darin aus[drückt], das[s] ... der Beeinflussung des Gesundheitszustandes geringe Möglichkeiten eingeräumt werden“ (Sperlich und Mielck 2003: 176). Es ist somit anzunehmen:

\section{H4b: Negative oder geringe Kontrollüberzeugungen sind in unteren Bildungsgruppen stärker ausgeprägt und können (in Lebensstilen objektiviert) einen Beitrag zur Aufklärung bil- dungsspezifischer Frühgeburtsrisiken leisten.}

In diesem Zusammenhang besitzt der Klassengeschmack als ,inkorporierte Kultur [oder] Körper gewordene Klasse“ (Bourdieu 1987: 307) außerordentliches handlungsleitendes Potenzial und dient sogleich als „...Erzeugungsformel, die dem Lebensstil zugrunde liegt...“ (Bourdieu 1987: 283). Der Lebensstil bezieht sich auf konkrete Praxisformen, welche die Differenzen im Habitus zwischen den Klassen sichtbar machen (Bourdieu 1987: 217, 405). Die aus medizinischer Sicht förderlichen, riskanten und schädlichen Verhaltensweisen (Troschke 2006: 529) korrespondieren mit dem distinguierten Lebensstil der oberen Klasse, dem zwischen den Praxen oberer und unterer Klasse einzuordnenden prätentiösen Lebensstil und der proletarischen Kultur der unteren Klasse (Bourdieu 1987: 286 ff.). ${ }^{26}$

Die Lebensführung während der Schwangerschaft ist von entscheidender Bedeutung für die Neugeborenengesundheit: „,[b]esondere Risiken, durch die ... [das Ungeborene] während der Schwangerschaft schweren gesundheitlichen Beeinträchtigungen ausgesetzt werden kann, stellen Rauchen, Alkohol- und Drogenkonsum sowie Medikamenteneinnahme dar" (Kitze und Kuske 2008: 418; vgl. Wulff 1997; Martius et al. 1998; Reime et al. 2006; Voigt et al. 2009; Calderon-Margali et al. 2009; O’Leary et al. 2009; Dew et al. 2007; Paulus 2006: 86;

\footnotetext{
${ }^{26} \mathrm{Zu}$ den gesundheitsfördernden Praktiken zählen im Allgemeinen eine ausreichende Energie- und Vitaminzufuhr, tägliche körperliche Aktivitäten, ein minimaler Tageskonsum von Alkohol, Tabak- und Drogenverzicht sowie ausreichende körperliche Hygiene, bedachtes Sexualverhalten, Spannungsregulation und die regelmäßige Inanspruchnahme von Vorsorgeuntersuchungen (Rütten et al. 2005: 13 f.; Bloomfield et al. 2008; RKI 2006; Hurrelmann 2006: 22). Gesundheitsschädigend ist ein dazu konträres Verhalten (Hurrelmann 2006: 74), während eine in diesem Sinne gelegentlich schädliche Gesundheitspraxis als riskant gilt (Troschke 2006: 529).
} 
Huch 2006: 201 ff.). Zwar stellt die überwiegende Mehrheit der Frauen, sobald sie um ihre Schwangerschaft wissen, gesundheitsschädigende Verhaltensweisen ein (Voigt et al. 2006b; Harrison und Sidebottom 2009; Strandberg-Larsen et al. 2008), allerdings lässt sich auch hier ein Bildungsgradient verzeichnen. So berichten Voigt und Kollegen (2006b: 271) unter Verwendung von deutschen Perinataldaten, dass ,20\% der Schwangeren auch nach dem Bekanntwerden der Schwangerschaft weiter [täglich] rauchten“ - darunter anteilig mehr Frauen mit niedrigem Bildungsstand (Brüning 2005, zit. nach Voigt et al. 2006b: 275; siehe auch Lampert und List 2010: 4). Darüber hinaus erklärt Rauchen während der Schwangerschaft einen beträchtlichen Teil des bildungsabhängigen Frühgeburtsrisikos (ca. 43 \%, van den Berg et al. 2012). Uneinheitlich ist hingegen der Forschungsstand zur Frage, welche Gruppen während der Schwangerschaft häufiger Alkohol konsumieren. Bisherige Studien berichten sowohl positive wie negative soziale Gradienten (Walker et al. 2011) oder finden gar keine sozialen Stratifikationseffekte (Strandberg-Larsen et al. 2008). ${ }^{27}$

Die Gründe für die Ausübung schädlichen Gesundheitsverhaltens könnten ähnliche sein, wie sie Bourdieu im Allgemeinen anführt, nämlich, dass Angehörige der unteren Klassen ihrer Gesundheit aufgrund ihres durch „Resignation“ bedingten Notwendigkeitsgeschmacks keine Zeit widmen und „sich selbst nicht hoch genug schätzen“ (Bourdieu 1987: 595, 596, Hervorhebung im Original; vgl. Williams 1995). Analog erweist sich auch die freiwillige Teilnahme an der in den Mutterschafts-Richtlinien gesetzlich geregelten Schwangerenvorsorge, welche bis zum achten Schwangerschaftsmonat ,monatlich eine Untersuchung und in den letzten zwei Schwangerschaftsmonaten jeweils zwei Untersuchungen im Abstand von 14 Tagen“ vorsieht (Simoes et al. 2003: 541), als bildungsselektiv. Die Inanspruchnahme der insgesamt 12 Standardvorsorgeuntersuchungen verlangt von den Teilnehmerinnen Zeit und setzt entsprechendes Wissen darüber voraus, dass Schwangerenvorsorge existiert, kostenlos und von hoher Gesundheitsrelevanz ist. Empirische Evidenzen unterstreichen die Bedeutsamkeit dieses präventiven Angebots, so haben Schwangere mit Standardvorsorge (mehr als 9 Untersuchungen) im Vergleich zu jenen mit geringerer Inanspruchnahme, überwiegend Frauen mit niedrigem Schulabschluss, ein deutlich geringeres Frühgeburtenrisiko (für Deutschland Simoes et al. 2003; Reime et al. 2006; Koller et al. 2008; für internationale Befunde Dew et al. 2007; Krueger und Scholl 2001). ${ }^{28}$

Abschließend kann ein klassenspezifisches „Körperbild“ von ursächlicher Bedeutung für das Gesundheitsverhalten sein (Bourdieu 1987: 305). Weil untere Klassen die Kraft eines Körpers mehr wertschätzen als seine Gestalt, führen sie ihrem Körper - „unwiderlegbarste Objektivierung des Klassengeschmacks“ - eher billige und nahrhafte Lebensmittel zu, während obere Klassen leichte und gesundheitsfördernde Produkte bevorzugen (Bourdieu

\footnotetext{
${ }^{27}$ Ein schichtabhängiger Tabakkonsum gilt als abgesichert. Im Gegensatz dazu steigt jedoch der Alkoholkonsum mit zunehmender Bildungs- oder Einkommensstärke der Frauen (Henkel et al. 2003).

${ }^{28}$ Anzumerken ist, dass auch Wegkosten anfallen können, die bei unteren Bildungsgruppen stärker ins Gewicht fallen.
} 
1987: 307). Zu einem gesundheitsförderlichen Umgang mit dem Körper zählt auch dessen sportaktiver Gebrauch in der Freizeit, welcher ebenso vom Verhältnis zum eigenen Körper abhängt. ${ }^{29}$ Aufgrund eines stärker instrumentellen Verhältnisses zum eigenen Körper sind untere Klassen eher solchen Betätigungen zugeneigt, die „höchsten Krafteinsatz ... erfordern“ (Bourdieu 1987: 339). Im Hinblick auf die Schwangerschaft ist bereits bekannt, dass ein ungesundes Ernährungsverhalten in Form einer Mangel- oder Überernährung von „Mikronährstoffen“ sowie das Ausüben von Risikosportarten (Tauchen, Reiten, Gewichtheben, oder Mannschafts- und Kontaktsportarten) wehenfördernd wirkt (Bung 2006). Zuletzt resultieren ungesunde Ernährungsweisen und Bewegungsmangel häufiger in Übergewicht (RKI 2005). Auch der Body-Mass-Index (BMI), geeigneter Indikator für das Gesundheitsverhalten (Jungbauer-Gans 2006) und das Körperbild, spielt eine bedeutsame Rolle für die Schwangerschaftsdauer. Wie Bergmann und Dudenhausen (2003) auf Grundlage der Berliner Perinataldaten zeigen, erhöht ein vorschwangerschaftlicher BMI kleiner als 19,8 oder größer als 28 $\mathrm{kg} / \mathrm{m}^{2}$ das Frühgeburtsrisiko deutlich (siehe auch Hänseroth et al. 2007: 34; für internationale Evidenz vgl. Svensson et al. 2009).

Die Überlegungen zu den potenziell schwangerschaftsverkürzenden Habitus und Lebensstilursachen zusammenfassend, lassen sich zwei weitere Hypothesen aufstellen:

H4c: Mit steigendem Bildungsgrad sollte, aufgrund der besseren Vertrautheit mit gesundheitsförderlichen Verhaltensweisen, einer geringeren Risikobereitschaft und stärker ausgeprägten Kontrollüberzeugungen, die Wahrscheinlichkeit für gesundheitsriskantes Verhalten während der Schwangerschaft sinken.

H4d: Eine schwangerschaftsverkürzende Wirkung riskanter Einstellungen (hohe Risikobereitschaft, negative Kontrollüberzeugungen) oder Gesundheitspraxen (geringe Schwangerenvorsorge, Tabakkonsum, unregelmäßige sportliche Betätigung und zu hoher oder zu niedriger Body-Mass-Index) vorausgesetzt, sollte sich bei einer multivariaten Betrachtung eine deutliche Reduzierung des Bildungsgradienten im Frühgeburtsrisiko feststellen lassen.

\subsection{Datenbasis und Variablenkonstruktion}

Die empirischen Analysen basieren auf den Daten des Sozio-oekonomischen Panels (SOEP, 2003-2011, v28), welches mit dem seit 2003 eingesetzten Zusatzfragebogen „Mutter und Kind“ (bioage01) Informationen zur Schwangerschaft und Neugeborenengesundheit bereitstellt (vgl. Rosenbladt et al. 2003: 11; Schupp et al. 2010: 169; Haisken-DeNew und Frick 2005). In methodischer Hinsicht orientieren wir uns an einem prospektiven Studiendesign (siehe hierzu auch Currie und Moretti (2003) oder Peters (2010)), um ein genaues Abbild der pränatalen Lebensbedingungen und Lebensstile zu gewinnen. Mithilfe der von der SOEPGruppe bereitgestellten (nicht im Zusatzfragebogen enthaltenen) Variable bcpregy (Schwan-

\footnotetext{
${ }^{29}$ Die Formung des Körpers vollzieht sich natürlich auch im Arbeitsprozess (Bourdieu 1987: 307), mit dem unterschiedliche „körperliche und psychische Belastungen“ (Lampert und Ziese 2005: 52) verbunden sein können.
} 
ger beim Personeninterview) identifizieren wir jene Mütter, für welche Personen- und Haushaltsinformationen während der Schwangerschaft vorliegen. Die Gruppe der nicht während der Schwangerschaft Befragten wird von der empirischen Analyse ausgeschlossen. ${ }^{30}$ Insgesamt verbleiben damit, von ursprünglich 1959 Teilnehmerinnen des Mutter-KindFragebogens, 1316 Mütter im Datensatz. Die Mehrheit dieser Frauen (1073) ist aufgrund wiederholter Befragungsteilnahmen bei erneuter Kindesgeburt oder Mehrlingsentbindungen mehrfach enthalten. Für die Gewährleistung unabhängiger Beobachtungseinheiten erfolgt daher eine Clusterung der Standardfehler über die Personennummern der Mütter (für ein analoges Vorgehen vgl. Kemptner und Marcus 2012). Im Folgenden erläutern wir die Konstruktion der zentralen abhängigen und unabhängigen Variablen.

Frühgeburt. Die Dauer der Schwangerschaft ist im „Mutter und Kind“ Fragebogen mit der Frage „In der wievielten Schwangerschaftswoche wurde Ihr Kind geboren?“ abgebildet. Da eine eigenständige Lebensfähigkeit erst ab der 37. Woche vollständig erreicht ist (vgl. Rettwitz-Volk 2003: 145; Moore und Persaud 2007: 113), unterscheidet unsere abhängige Variable, in Anlehnung an die Frühgeburtsdefinition der WHO und UNICEF (2004: 2), zwischen Frühgeborenen $(=1)$ und Neugeborenen, welche 37 Wochen und länger im Mutterleib verblieben sind $(=0)$.

Kulturelles Kapital. Informationen über die formale Bildungsqualifikation sind in den wellenspezifischen Personendatensätzen hinterlegt. Wir unterscheiden drei Bildungskategorien (Fach-Abitur $=0$, Realschulabschluss $=1$ und Hauptschulabschluss oder kein Schulabschluss =2). Frauen, die zum Befragungszeitpunkt noch keinen Abschluss innehatten, sich aber in einer Bildungseinrichtung befanden, wurden der gegenwärtigen Schulform zugeordnet.

Ökonomisches Kapital. Das Einkommen wird nach der neuen Netto-Äquivalenzskala der OECD berechnet, indem das Haushaltsnettoeinkommen durch die Anzahl der gewichteten Haushaltsmitglieder dividiert wird (siehe hierzu Lampert und Kroll 2005: 5). Die dichotome Variable Armut nimmt den Wert 1 an, sofern das Haushaltsbudget weniger als $60 \%$ des Median beträgt (vs. $0=\geq 60 \%$ des Median) (siehe hierzu Hurrelmann 2006: 37). ${ }^{31}$

Soziales Kapital. Die Quantität des sozialen Kapitals operationalisieren wir über das Vorhandensein eines Partners (Nein $=1, \mathrm{Ja}=0$ ) und über die Besuchshäufigkeit von Familienangehörigen, Freunden und Nachbarn (Monatlich, Selten oder Nie =2, mind. einmal wöchent-

\footnotetext{
${ }^{30}$ Robustheitsanalysen mit allen Teilnehmerinnen der Mutter-Kind-Befragung (fallweiser Ausschluss, $\mathrm{N}=1780$ ) weisen im Vergleich zu der während der Schwangerschaft befragten Gruppe (fallweiser Ausschluss, N = 1247) nur auf geringfügige, inhaltlich aber nicht wesentliche Ergebnisunterschiede hin. Abweichungen lassen sich jedoch für die Gruppe der nicht während der Schwangerschaft befragten Mütter (fallweiser Ausschluss, N = 533) beobachten: Keine der Modellvariablen ist signifikant mit der Kriteriumsvariable assoziiert.

${ }^{31}$ Die Entscheidung für dieses Einkommensmaß liegt darin begründet, dass insbesondere Mütter aus der untersten Einkommensschicht ein erhöhtes Frühgeburtsrisiko haben (vgl. Blumenshine et al. 2010).
} 
lich $=1$, täglich =0). ${ }^{32}$ Die Qualität des sozialen Kapitals bilden wir indirekt über die formale Bildungsqualifikation des Partners ab ((Fach-)Abitur $=0$, Realschulabschluss $=1$, maximal Hauptschulabschluss $=2$ ).

Risikobereitschaft. Die Risikowahrnehmung erfassen wir mit der Frage „Wie schätzen Sie sich persönlich ein: Sind Sie im Allgemeinen ein risikobereiter Mensch oder versuchen Sie, Risiken zu vermeiden?“ (elfstufige Skala, $0=$ Gar nicht risikobereit bis $10=$ Sehr risikobereit). In unseren Analysen geht diese Variable metrisch ein. ${ }^{33}$

Kontrollüberzeugungen ${ }^{34}$. Kontrollüberzeugungen bilden wir mit den zwei metrischen Variablen „Was man im Leben erreicht, ist in erster Linie eine Frage von Schicksal oder Glück“ und „Ich habe wenig Kontrolle über die Dinge, die in meinem Leben passieren“ (siebenstufige Skala, 1 = Stimme überhaupt nicht zu bis $7=$ Stimme voll zu) ab. ${ }^{35}$

Tabakkonsum. Der Tabakkonsum ist mit der Frage „Rauchen Sie gegenwärtig, seien es Zigaretten, Pfeifen oder Zigarren?“ im Personenfragebogen enthalten. In den Analysen unterscheiden wir, ob Mütter während der Schwangerschaft Tabak konsumierten (=1) oder nicht $(=0)$.

Sportliche Betätigung. Aufgrund unterschiedlicher Antwortskalen und geringer Zellbesetzungen stufen wir eine sportliche Betätigung während der Schwangerschaft als regelmäßig (=0) ein, wenn diese mindestens einmal im Monat ausgeführt wurde. Demgegenüber gehen wir bei gelegentlicher, seltener oder keiner Ausübung von einer unregelmäßigen sportlichen Betätigung (=1) aus.

Ärztliche Inanspruchnahme (Becker und Stolberg 2013). Die Inanspruchnahme der Schwangerenvorsorge ist nicht im SOEP verankert. Eine ungefähre Generierung dieser Variable ist über Fragen zur ärztlichen Inanspruchnahme („Haben Sie in den letzten 3 Monaten Ärzte aufgesucht?") und zum Schwangerschaftsmonat beim Personeninterview (Variable: bcpregmo) möglich. Die Frage zum Arztbesuch umfasst die Antwortmöglichkeiten „Keinen Arzt in Anspruch genommen“ oder „Zahl aller Arztbesuche in den letzten 3 Monaten“. Die ärztliche Inanspruchnahme während der Schwangerschaft wird positiv im Sinne einer adäqua-

\footnotetext{
${ }^{32}$ Diese Variable ist ein Konstrukt aus drei Einzelindikatoren zur Besuchshäufigkeit. Aufgrund differierender Antwortskalen und geringer Zellbesetzungen wurden die Angaben zu einer dreistufigen Variable zusammengefasst.

${ }^{33}$ Statt der allgemeinen Risikowahrnehmung wären gemäß des Theorierahmens Indikatoren zur gesundheitsbezogenen Risikowahrnehmung besser geeignet. Entsprechende Angaben wurden jedoch ausschließlich in den Jahren 2004 und 2009, Angaben zur allgemeinen Risikowahrnehmung fast durchgängig erhoben. Wir haben uns daher für letztere Variable entschieden, auch aufgrund der Annahme, dass sich die eigene Risikobereitschaft ändern kann, sobald eine Frau um ihre Schwangerschaft weiß.

${ }^{34}$ Angaben zu den Kontrollüberzeugungen wurden ausschließlich in den Jahren 2005 und 2010 erfasst. Da solche Einstellungen als relativ stabil gelten (vgl. Mirowsky und Ross 2007) und adäquatere Maße nicht zur Verfügung standen, wurden für die in den Jahren 2003 bis 2007 befragten Mütter die Werte des Jahres 2005 übertragen. Analog bekamen Frauen, die in den Jahren 2008 bis 2011 den „Mutter und Kind“ Fragebogen beantworteten, den Einstellungswert aus dem Jahr 2010 zugewiesen.

${ }^{35}$ Eine Indexbildung ist mit einem Cronbachs Alpha von 0,5 (Wert gerundet), eine Güte, die unter der geforderten Reliabilität von 0,8 liegt (Diekmann 2005: 220), nicht zu empfehlen.
} 
ten Teilnahme an der Schwangerenvorsorge angesehen $(=0)$, wenn im ersten bis dritten Schwangerschaftsmonat (SSW: 0-16) mindestens einmal ein Arzt aufgesucht wurde, im vierten Schwangerschaftsmonat (SSW: 17-20) mindestens zweimal die ärztliche Inanspruchnahme und ab dem fünften Schwangerschaftsmonat (SSW: ab 21) mindestens dreimal die ärztliche Inanspruchnahme erfolgte. Als inadäquat (=1) gilt die Inanspruchnahme dann, wenn kein Arzt aufgesucht wurde, oder im vierten Schwangerschaftsmonat lediglich einmal und ab dem fünften Schwangerschaftsmonat weniger als dreimal die ärztliche Inanspruchnahme erfolgte.

Body-Mass-Index. In Anlehnung an den bisherigen Forschungsstand unterscheidet die kategoriale Variable zum vorschwangerschaftlichen Body-Mass-Index zwischen normal- bis übergewichtig $\left(0=19,8\right.$ bis $\left.28,0 \mathrm{~kg} / \mathrm{m}^{2}\right)$, untergewichtig $\left(1=\leq 19,7 \mathrm{~kg} / \mathrm{m}^{2}\right)$ und stark übergewichtig $\left(2=\geq 28,1 \mathrm{~kg} / \mathrm{m}^{2}\right)$. Diese Klassifikation weicht geringfügig von der Definition der WHO (1998) ab.

Kontrollvariablen. In den Analysen wird außerdem kontrolliert für eine Reihe von kindlichen (Geschlecht: 0 = Junge, 1 = Mädchen; Geburtsjahr: metrisch; Mehrling: $0=$ Nein, $1=\mathrm{Ja}$ ) und mütterlichen Eigenschaften (Migrationshintergrund: $0=$ Nein, $1=$ Direkt, 2 Indirekt; Alter zum Zeitpunkt der Schwangerschaft: $0=22$ bis 34 Jahre, $1=\leq 21$ Jahre, $2=\geq 35$ Jahre; Parität: 0 = Zweitgebärende, 1 = Erstgebärende, $2=$ Mehrgebärende; Schwangerschaftsplanung: $0=\mathrm{Ja}, 1=$ Nein, $2=\mathrm{Ja}$, mit medizinischer Unterstützung; Gesundheitszustand im letzten Schwangerschaftsdrittel (additiver Index aus physischem und seelischem Befinden): 0 = Sehr gut, 1 = Gut, 2 = Zufriedenstellend/Schlecht). Schließlich berücksichtigen wir die regionale Herkunft $(0=$ West, $1=$ Ost $)$ und die Krankenversicherungsart ( 0 = Gesetzlich, 1 = Privat $)$.

In der vorliegenden Arbeit sind fehlende Angaben im kulturellen Kapital (3,88 \%), ökonomischen Kapital (5,24 \%), der Qualität sozialen Kapitals ${ }^{36}$ (7,37\%), den Kontrollüberzeugungen (7,37 \% bzw. 6,91\%), der ärztlichen Inanspruchnahme $(0,23 \%)$, sowie in der Parität $(0,08 \%)$, Schwangerschaftsplanung $(0,61 \%)$, dem Gesundheitszustand im letzten Schwangerschaftsdrittel $(0,07 \%)$ und der Krankenversicherungsart $(0,15 \%)$ enthalten.

Da nicht durchgängig jedes Jahr erhoben, verzeichnen einige Variablen noch höhere prozentuale Anteile fehlender Werte. ${ }^{37}$ So erfolgte die Erfassung der Besuchshäufigkeit von Familienangehörigen, Freunden und Nachbarn in den Jahren 2001, 2003, 2005 sowie 2007 bis 2009 (44,68 \% fehlend), der persönlichen Risikobereitschaft in den Jahren 2004, 2006, 2008, 2009, 2010 und 2011 (50,23 \% fehlend), des Tabakkonsums in den Jahren 2001, 2002, 2004, 2006, 2008 und 2010 (52,89\% fehlend), der sportlichen Betätigung in den Jahren 2001, 2003 bis 2005, 2007 bis 2009 und 2011 (31,84 \% fehlend), und die zur Berechnung des BodyMass-Indexes benötigten Variablen (Körpergewicht und Körpergröße) ausschließlich jedes

\footnotetext{
${ }^{36} 14,59 \%$ sind sogenannte hard missings, die nicht imputiert werden dürfen und in den Analysen über eine dichotome Variable kontrolliert werden.

${ }^{37}$ Die Variablen zum Ernährungsverhalten und Alkoholkonsum wurden aufgrund der hohen Anzahl fehlender Werte (über $70 \%$ ) nicht in den Analysen berücksichtigt.
} 
zweite Befragungsjahr ab 2002 (58,97 \% fehlend). Angesichts der kleinen Stichprobengröße und der überwiegend vom Befragungsjahr abhängigen fehlenden Werte, erfolgt eine Schätzung mithilfe der multiplen Imputationsmethode ${ }^{38}$ (StataCorp 2009: 3). Bei diesem Vorgehen werden fehlende Angaben m-mal über zuvor festgelegte Prädiktoren geschätzt (hier: Modellvariablen; Kulturelles Kapital/Ökonomisches Kapital/Besuchshäufigkeit/Qualität sozialen Kapitals/persönliche Risikobereitschaft/Tabak-konsum/Sportliche Betätigung zum Befragungszeitpunkt; Geburtsgewicht; Schwangerschaftsjahr und Schwangerschaftsmonat zum Befragungszeitpunkt; Angaben zur Befragungsart und zum Geschlecht der befragenden Person), die anschließend im sogenannten ,pooling step“ (StataCorp 2009: 3) zusammengefasst in die multivariaten Analysen eingehen (Sterne et al. 2009: 2; Wood et al. 2008; Lüdtke et al. 2007). Aufgrund hoher Ausfallquoten in den Variablen persönliche Risikobereitschaft, Tabakkonsum und Body-Mass-Index erfolgt eine 100-malige Wiederholung des Schätzprozesses. $^{39}$

\subsection{Ergebnisse}

\subsubsection{Die Modellvariablen nach mütterlichem Kulturkapital}

Basis des folgenden deskriptiven Überblicks über die Verteilung zentraler Modellvariablen nach mütterlichem Kulturkapital, ist der erste imputierte Datensatz. ${ }^{40}$ Tabelle 5.1 gibt Auskunft darüber, ob die formale Bildung der Frauen mit der Schwangerschaftsdauer assoziiert ist (Hypothese H1). Zunächst ist ersichtlich, dass insgesamt 8,89\% aller hier betrachteten Neugeborenen zu früh auf die Welt kommen. Die Frühgeburtsrate weicht damit nicht wesentlich vom bundesdeutschen Durchschnittswert der letzten Jahre ab (vgl. hierzu BQS 2012).

Tabelle 5.1: Verteilung der Frühgeburt nach mütterlichem Kulturkapital.

\begin{tabular}{llllll}
\hline Frühgeburt & \multicolumn{3}{l}{ Ja } & & \multicolumn{2}{l}{ Nein } & \\
& Prozent & (Anzahl) & & Prozent & (Anzahl) \\
\hline Kulturelles Kapital der Mutter** & & & & \\
(Fach-)Abitur & 7,86 & $(44)$ & & 92,14 & $(516)$ \\
Realschulabschluss & 7,59 & $(39)$ & 92,41 & $(475)$ \\
Hauptschulabschluss & 14,05 & $(34)$ & & 85,95 & $(208)$ \\
\hline $\mathrm{N}$ & 8,89 & $(117)$ & 91,11 & $(1.199)$ \\
\hline
\end{tabular}

** $p<0,01 ; p=\chi^{2}$. Abweichungen von $100 \%$ sind durch Aufrundungen möglich;

Quelle: SOEP 2003-2011; eigene Berechnungen auf Basis des ersten imputierten Datensatzes

\footnotetext{
${ }^{38}$ „Für die Berichterstattung haben wir auf die empfohlenen Richtlinien von Sterne et al. (2009) zurückgegriffen (siehe Sterne et al. 2009)“.

${ }^{39}$ Zur m-maligen Schätzung bei hoher Anzahl fehlender Werte, siehe StataCorp 2009: 11. Die Berechnung erfolgte mit dem Befehl ,mi impute chained“ (Stata 12.0).

${ }^{40}$ Für Zusammenhänge zwischen dem mütterlichen Kulturkapital und den Kontrollvariablen siehe Tabelle A 5.2 oder Tab. A1, online-Anhang; http://www.uni-koeln.de/kzffs/materialien/KS-67-2-Stolberg_Becker.pdf.
} 
In Übereinstimmung mit unserer Erwartung zeigen sich zudem bedeutsame Bildungsdisparitäten in der Frühgeburtsprävalenz zum Nachteil der untersten Bildungsgruppe: Entbinden etwas weniger als $8 \%$ aller Frauen mit weiterführender Bildung ihre Kinder zu früh, ist der Vergleichswert für Hauptschulabsolventinnen mit $14 \%$ signifikant höher $(\mathrm{p}<0,01)$. Interessanterweise liegen Frauen mit hohem und mittlerem Abschluss gleich auf, was Bourdieus Linearitätsannahme widerspricht, nach welcher eine „Vertikalverlagerung“ nach oben mit einer graduellen Abschwächung der Ungleichheit einhergehen müsste (Bourdieu 1987: 220, Hervorhebung im Original; vgl. 1987: 212 ff.).

Es stellt sich nun die Frage, welche Faktoren hinter diesem gesundheitlichen Ungleichheitsphänomen stehen. Wie im Theorieabschnitt diskutiert, dürften die Ursachen auf der Verhältnis-, Einstellungs- und Verhaltensebene zu finden sein. Um statistisch als Mediatoren in Frage zu kommen, müssten sich zwischen den Bildungsgruppen signifikante Unterschiede zeigen. Tabelle 5.2 stellt hierzu die Ergebnisse dar. Mit Blick auf die ökonomischen Verhältnisse tragen Hauptschulabsolventinnen mit einem monatlichen Netto-Äquivalenzeinkommen von $1142 €$ das größte Armutsrisiko, gefolgt von Schwangeren mit mittlerem Bildungsabschluss (1408 €). Darüber hinaus ist das relative Risiko (OR) von Frauen mit Hauptschulabschluss, im Vergleich zu jenen mit (Fach-)Abitur, viermal so groß über weniger als $60 \%$ des Medianeinkommens zu verfügen. ${ }^{41}$ Für Realschulabsolventinnen stellt sich die Armutsproblematik mit einem OR von 2,6 vergleichsweise weniger dramatisch dar.

Analog verhält es sich mit der sozialen Einbindung: Je höher die Bildung schwangerer Frauen, desto eher leben diese in einer Partnerschaft ( $p<0,01)$. Dieser Befund steht im Einklang mit bisherigen Studien, dass Alleinelternschaft (Statistisches Bundesamt 2010) oder Partnerlosigkeit (Lengerer 2012) im untersten Bildungssegment verbreiteter ist. Auch finden sich eindeutige deskriptive Belege dafür, dass Partnerwahl ,auf Augenhöhe“" stattfindet (Skopek et al. 2009: 1), wie der höchstsignifikante Zusammenhang zwischen weiblichem und männlichem $^{42}$ Bildungsgrad belegt. Ferner zeigt sich, dass Frauen mit einem Realschulabschluss gegenüber (Fach-)Abiturientinnen eine fast vierfach so hohe relative Chance haben $((29,20 / 70,81) /(9,50 / 90,49))$, eine Partnerschaft mit einem Hauptschulabsolventen zu führen. Dieser Ressourcennachteil ist bei Frauen mit einem Hauptschulabschluss und einem OR von $14,5((60,49 / 39,52) /(9,50 / 90,49))$ deutlich höher ausgeprägt (vgl. hierzu auch Kemptner und Marcus 2012).

\footnotetext{
${ }^{41}$ Das Odds Ratio ergibt sich, indem man die Armutschancen der jeweiligen Bildungsgruppen zueinander ins Verhältnis setzt, also die Odds derjenigen mit Hauptschulabschluss $(26,45 / 73,55)$ durch jene mit mindestens (Fach-)Abitur (6,96/93,04) dividiert.

${ }^{42}$ An dieser Stelle sei jedoch darauf hinzuweisen, dass es sich nicht zwangsläufig um den biologischen Vater handeln muss (vgl. Kemptner und Marcus 2012).
} 
Tabelle 5.2: Verteilung des ökonomischen- und sozialen Kapitals und des Gesundheitsverhaltens nach mütterlichem Kulturkapital

\begin{tabular}{|c|c|c|c|c|c|c|}
\hline \multirow[t]{2}{*}{ Kulturelles Kapital der Mutter } & \multicolumn{2}{|c|}{ Hauptschulabschluss } & \multicolumn{2}{|c|}{ Realschulabschluss } & \multicolumn{2}{|c|}{ (Fach-)Abitur } \\
\hline & Prozent & (Anzahl) & Prozent & (Anzahl) & Prozent & (Anzahl) \\
\hline \multicolumn{7}{|l|}{ Ökonomisches Kapital } \\
\hline Netto-Äquivalenzeinkommen ${ }^{\mathrm{a}}$ & \multicolumn{2}{|c|}{$\mathrm{M}=1.142,64 ; \mathrm{Std}=532,3$} & \multicolumn{2}{|c|}{$\mathrm{M}=1.408,56 ; \mathrm{Std}=654,4$} & \multicolumn{2}{|c|}{$\mathrm{M}=1.881,79 ; \mathrm{Std}=833,2$} \\
\hline \multicolumn{7}{|l|}{ Armut $* * *$} \\
\hline Nein ( $\geq 60$ Prozent des Median) & 73,55 & (178) & 83,27 & $(428)$ & 93,04 & (521) \\
\hline Ja (<60 Prozent des Median) & 26,45 & (64) & 16,73 & (86) & 6,96 & (39) \\
\hline \multicolumn{7}{|l|}{ Soziales Kapital } \\
\hline \multicolumn{7}{|l|}{ Partnerschaft** } \\
\hline Nein & 13,64 & (33) & 10,89 & (56) & 6,43 & (36) \\
\hline $\mathrm{Ja}$ & 86,36 & (209) & 89,11 & $(458)$ & 93,57 & (524) \\
\hline \multicolumn{7}{|c|}{ Besuch von Familie, Freunden, Nachbarn** } \\
\hline Täglich & 11,98 & (29) & 8,37 & (43) & 5,00 & (28) \\
\hline Wöchentlich & 54,55 & (132) & 57,39 & (295) & 63,93 & (358) \\
\hline Seltener & 33,47 & $(81)$ & 34,24 & (176) & 31,07 & (174) \\
\hline \multicolumn{7}{|l|}{ Bildung des Partners*** } \\
\hline Hauptschulabschluss & 60,49 & (124) & 29,20 & (127) & 9,50 & (46) \\
\hline Realschulabschluss & 25,37 & $(52)$ & 44,83 & (195) & 22.31 & $(108)$ \\
\hline (Fach-)Abitur & 14,15 & (29) & 25,98 & (113) & 68,18 & (330) \\
\hline (Anzahl fehlender Werte) & & (37) & & (79) & & (76) \\
\hline \multicolumn{7}{|l|}{ Habitus } \\
\hline Persönliche Risikobereitschaft ${ }^{\mathrm{b}}$ & \multicolumn{2}{|c|}{$\mathrm{M}=4,13 ; \mathrm{Std}=0,151$} & \multicolumn{2}{|c|}{$M=4,46 ; \operatorname{Std}=0,091$} & \multicolumn{2}{|c|}{$\mathrm{M}=4,37 ; \mathrm{Std}=0,087$} \\
\hline Leben ist abhängig von Glück ${ }^{\mathrm{a}}$ & \multicolumn{2}{|c|}{$\mathrm{M}=4,15 ; \mathrm{Std}=0,113$} & \multicolumn{2}{|c|}{$M=3,67 ; \mathrm{Std}=0,075$} & \multicolumn{2}{|c|}{$\mathrm{M}=3,01 ; \mathrm{Std}=0,059$} \\
\hline Wenig Kontrolle über das Leben ${ }^{\mathrm{a}}$ & \multicolumn{2}{|c|}{$\mathrm{M}=2,88 ; \mathrm{Std}=0,107$} & \multicolumn{2}{|c|}{$M=2,49 ; \mathrm{Std}=0,062$} & \multicolumn{2}{|c|}{$M=2,29 ; \mathrm{Std}=0,054$} \\
\hline \multicolumn{7}{|c|}{ Gesundheitsverhalten während der Schwangerschaft } \\
\hline \multicolumn{7}{|c|}{ Schwangerenvorsorge $* *$} \\
\hline Inadäquat & 21,49 & $(52)$ & 15,95 & $(82)$ & 11,61 & $(65)$ \\
\hline Adäquat & 78,51 & (190) & 84,05 & $(432)$ & 88,39 & (495) \\
\hline \multicolumn{7}{|l|}{ Tabakkonsum $* * *$} \\
\hline Nein & 54,13 & (131) & 77,24 & (397) & 90,18 & (505) \\
\hline $\mathrm{Ja}$ & 45,87 & (111) & 22,76 & (117) & 9,82 & (55) \\
\hline Sportliche Betätigung*** & & & & & & \\
\hline Unregelmäßig & 82,23 & (199) & 67,32 & (346) & 54,64 & (346) \\
\hline Regelmäßig & 17,77 & $(43)$ & 32,68 & (168) & 45,36 & (254) \\
\hline Body-Mass-Index* & & & & & & \\
\hline Untergewicht & 16,94 & (41) & 20,23 & (104) & 23,57 & (132) \\
\hline Normal- bis Übergewicht & 70,66 & (171) & 67,51 & (347) & 69,29 & (388) \\
\hline Starkes Übergewicht & 12,40 & (30) & 12,26 & (63) & 7,14 & (40) \\
\hline $\mathrm{N}$ & & (242) & & (514) & & $(560)$ \\
\hline
\end{tabular}

Quelle: SOEP 2003-2011; eigene Berechnungen auf Basis des ersten imputierten Datensatzes.

Anmerkungen: Abweichungen von $100 \%$ sind durch Aufrundungen möglich. ${ }^{a}$ Mittelwerttest mit signifikanten Unterschieden für alle Gruppen, ${ }^{b}$ Mittelwerttest mit signifikanten Unterschieden zwischen Haupt-und Realschulabschluss; *** $\mathrm{p}<0.001 ; * * \mathrm{p}<0.01 ; * \mathrm{p}<0.05 ;+\mathrm{p}<0.10 ; \mathrm{p}=\chi^{2} ; M$ Mittelwert; Std Standardabweichung. 
Im Hinblick auf den Zusammenhang zwischen kulturellem Kapital und dem regelmäßigen Besuch durch Familie, Freunde und Nachbarn $(\mathrm{p}<0,01)$ zeigt sich dagegen ein umgekehrter und damit unerwarteter Effekt: Der prozentuale Anteil von täglichen Kontakten nimmt mit steigendem Bildungsniveau kontinuierlich ab. Auf den ersten Blick zeigt sich auch für den Habitus (Einstellungsebene) ein unerwartetes Ergebnis. Entgegen Hypothese H4a, welche von einem umgekehrten Zusammenhang zwischen Bildung und Risikowahrnehmung ausgeht, geben Schwangere mit Hauptschulabschluss im Mittel eine geringere Risikobereitschaft an $(4,13)$ als jene mit Realschulabschluss $(4,46)$ oder (Fach-)Abitur $(4,37) .{ }^{43}$ Demgegenüber schwindet im Bereich der Denkschemata mit zunehmendem Bildungsgrad die Überzeugung, das eigene Leben wäre von Schicksal und Glück abhängig und entzöge sich der eigenen Kontrolle (Hypothese $\mathrm{H} 4 \mathrm{~b}$ ).

Schließlich lassen sich auf der Verhaltensebene statistisch bedeutsame und mit unseren Erwartungen konforme Bildungsunterschiede im Gesundheitshandeln schwangerer Frauen feststellen (Hypothese H4c): Die Wahrscheinlichkeit für Tabakkonsum und unregelmäßige sportliche Betätigung steigt mit sinkendem Bildungsgrad an ( $<<0,001)$.

Im Vergleich zur Gruppe der Frauen mit einem Hauptschulabschluss oder mittlerer Reife rauchen (Fach-)Abiturientinnen nicht nur seltener (9,82\%), sondern treiben während der Schwangerschaft auch regelmäßiger Sport $(45,36 \%)$ und nehmen häufiger an der Schwangerenvorsorge teil $(88,39 \%$; $<$ 0,01). Für den Body-Mass-Index lassen sich ebenfalls Bildungsunterschiede $(\mathrm{p}<0,05)$ erkennen. Im Vergleich zu Frauen mit (Fach-)Abitur haben Realschulabsolventinnen ein fast doppelt so hohes relatives Risiko $((12,26 / 87,74) /(7,14 / 92,86))$ ein Jahr vor der Schwangerschaft starkes Übergewicht zu haben. Bei Frauen mit Hautschulabschluss ist der OR, im Vergleich zu jenen mit Realschulabschluss, nur minimal höher $((12,40 / 85,14) /(7,14 / 92,86))$. Demgegenüber folgt Untergewicht einem umgekehrten Bildungsgradienten.

Wie in Tabelle A 5.1 zu erkennen, sind auch die anderen zwei Kapitalarten mit den mütterlichen Einstellungen und Gesundheitspraxen assoziiert. Allerdings fallen die Korrelationen im Vergleich zum Kulturkapital deutlich geringer aus, was auf die vermutete Schlüsselfunktion der Bildung verweist.

\subsubsection{Mediatoren bildungsabhängiger Frühgeburtsrisiken}

Welche Faktoren vermitteln nun den Zusammenhang zwischen Bildung und vorzeitiger Entbindung? Im Folgenden wird anhand logistischer Regressionen geprüft, ob sich die beobachteten Unterschiede im Frühgeburtsrisiko zwischen Frauen mit Hauptschulabschluss und weiterführender Bildung über Verhältnis-, Einstellungs- und Verhaltensgrößen aufklären lassen. Die Analyse folgt einem schrittweisen Vorgehen. In einem ersten Modell berücksichtigen wir neben der mütterlichen Bildung eine Reihe von (potenziell) konfundierenden Merkmalen, wie

\footnotetext{
${ }^{43}$ Auf den zweiten Blick ist dieses Ergebnis jedoch plausibel, stellt man in Rechnung, dass höher Gebildete, weil sie typischerweise über mehr Ressourcen verfügen, auch höhere Risiken eingehen können.
} 
z. B. dem Migrationshintergrund. Erweist sich der Bildungseffekt als stabil, untersuchen wir in weiteren Modellen, inwiefern die ökonomische und soziale Kapitalausstattung, gesundheitsrelevante Grundorientierungen (Risikobereitschaft, Kontrollüberzeugungen) und Lebensstilmerkmale (Tabakkonsum, sportliche Betätigung, ärztliche Inanspruchnahme während der Schwangerschaft und Body-Mass-Index) den Zusammenhang zwischen Frühgeburt und Bildung vermitteln. Um aussagekräftige Vergleiche zwischen hierarchischen Regressionsmodellen vorzunehmen, berichten wir durchschnittlich marginale Effekte (AME) (Wolf und Best 2010; Mood 2010; Auspurg und Hinz 2011). ${ }^{44}$ Für ein besseres Verständnis der multivariaten Befunde (Tabelle 5.3) gehen wir auch auf ausgewählte bivariate Zusammenhänge zwischen den Erklärungsgrößen und der Frühgeburt ein (siehe univariate Statistik, Tabelle A 5.1).

Wie Modell 1 (Tabelle 5.3) zeigt, verliert der für Hauptschulabsolventinnen ausgewiesene Bruttoeffekt (AME =0,047, p <0,05, Tabelle A 5.2) unter Kontrolle von demografischen Drittvariablen nur geringfügig an Stärke $(\mathrm{AME}=0,045, \mathrm{p}<0,10)$. Ob nun die ökonomische und soziale Kapitalausstattung sowie die Lebensstilmerkmale den Zusammenhang zwischen Bildung und Frühgeburt vermitteln, überprüfen wir schrittweise mit den Modellen 2 und 3 (Tabelle 5.3). Hypothese H2a, welche einen signifikanten Einfluss ökonomischer Ressourcenknappheit auf vorzeitige Entbindungen voraussetzt, kann univariat (AME $=0,034$, $\mathrm{p}<0,10$, Tabelle A 5.2) aber nicht multivariat bestätigt werden: Schwangere, die monatlich mit weniger als $60 \%$ des Einkommensmedian wirtschaften, haben ein tendenziell erhöhtes (genauer 2,8 \%,p = 0,173) Frühgeburtsrisiko (Modell 2, Tabelle 5.3). Nach Berücksichtigung des Armutsindikators geht der negative Geburtseffekt auf eine geringe Kulturkapitalausstattung zurïck (AME $=0,039, \mathrm{p}<0,10$ ), was im Einklang mit Hypothese H2b steht. Die Abschwächung des Bildungseffekts durch die Berücksichtigung des Armutsindikators fällt dabei erwartungsgemäß gering aus. Wie im Theorieteil argumentiert könnte dieses Ergebnis auf eine dem kulturellen Kapital untergeordnete Rolle des ökonomischen Kapitals hinweisen. Weiterführende Analysen mit anderen Einkommensspezifikationen (metrische Skala, Median, Quartile, Quintile) erhärten diese Vermutung: Keiner der alternativen Einkommensmaße ist signifikant mit der Frühgeburt assoziiert (Ergebnisse nicht dargestellt). Dieser Befund ähnelt den Ergebnissen einer älteren Studie für Deutschland, der gemäß für West- und Ostdeutschland konsistente Bildungseinflüsse auf das Schwangerschaftsergebnis (Frühgeburt, Geburtsgewicht) beobachtet wurden, Einkommenseffekte hingegen nur in Ostdeutschland (Blumenshine et al. 2010: 267).

In Modell 3 wird zusätzlich das mütterliche Sozialkapital in die Analyse aufgenommen. Die Vermutung, eine gute Sozialkapitalausstattung könnte über Prozesse sozialer Unterstützung (Anwesenheit eines Partners, regelmäßige soziale Kontakte, Hypothese H3a) oder Kontrolle (im Falle eines gesundheitskulturell hoch gebildeten Partners, Hypothese H3b) Bildungsnachteile in der Schwangerschaftsdauer aufklären (Hypothese H3c), trifft mit Einschränkung nur für die Bildung des Partners zu. Zwar geht unter Hinzunahme des quantitati-

\footnotetext{
${ }^{44}$ Die Berechnung durchschnittlich marginaler Effekte erfolgt mit dem Befehl mimrgns.
} 
ven und qualitativen Sozialkapitals in Modell 3 (Tabelle 5.3), der durchschnittlich marginale Effekt bei Hauptschulabsolventinnen deutlich zurück (AME =0,022) und ist mit $p=0,411$ (Wert nicht in Tabelle) auch nicht mehr signifikant. Für die unterschiedlichen Sozialkapitalmaße zeigen sich jedoch teils unerwartete, teils nur tendenziell bedeutsame Ergebnisse. So liegt der Schätzer für alleinstehende Frauen in Modell 3 nahe null (AME $=0,007)$ und eine wöchentliche $(\mathrm{AME}=-0,039)$ oder noch seltenere $(\mathrm{AME}=-0,041)$ Besuchshäufigkeit ist im Vergleich zur Referenzgruppe (tägliches Beisammensein mit Familie, Freunden und Nachbarn) sogar mit einem geringeren Frühgeburtsrisiko assoziiert. Letzteres könnte durchaus auf „Belastungen durch soziale Beziehungen“ verweisen (Jungbauer-Gans 2002: 129; vgl. Wolf 2006). Es ist aber ebenso denkbar, dass Schwangere mit einer besonders hohen Interaktionsdichte im Alltag aus gesundheitlichen Gründen auf besondere soziale Unterstützung angewiesen sind. Aufgrund von Datenbeschränkungen können wir diesem Befund nicht weiter nachgehen.

Zuletzt steht die Bildung des Partners, in Richtung und Stärke mit der mütterlichen Bildung vergleichbar, in einem mehr (univariat: $p<0,05$, Anhang Tabelle A 5.2) oder weniger (multivariat: $\mathrm{p}<0,20$, Tabelle 5.3) statistisch bedeutsamen Zusammenhang mit dem Ereignis einer Frühgeburt (vgl. hierzu auch Blumenshine et al. 2011; Becker und Stolberg 2013). Eine vollständige Erklärung des bildungsspezifischen Frühgeburtsrisikos wird durch das Ressourcenmodell (Modell 3) also nicht geleistet, wie an den Residualeffekten für die mütterliche Bildung abzulesen ist.

In Anlehnung an die theoretischen Überlegungen zur Verwobenheit von Kulturkapital und Lebensstil (mit dem Habitus als unsichtbares Bindeglied) ist dies nicht überraschend. Ob die verbleibenden Bildungseinflüsse nun mithilfe übergreifender Einstellungen (Habitusdimensionen: persönliche Risikobereitschaft und Kontrollüberzeugungen) und deren Objektivierung im Lebensstil (Tabakkonsum, inadäquate Vorsorge, unregelmäßige sportliche Betätigung, Body-Mass-Index) aufgeklärt werden, zeigen Modell 4 (ohne Kontrolle der Ressourcenausstattung) und Modell 5 (mit Kontrolle der Ressourcenausstattung). Insgesamt bleibt die Reduktion der Frühgeburtsrisiken für Schwangere mit der geringsten Bildungsqualifikation hinter unseren theoretischen Erwartungen (Hypothese H4d) zurück. So offenbaren zwar die Bruttoeffekte aus Tabelle A 5.2 (Anhang), dass die eigene Überzeugung, das Leben sei von Glück abhängig (AME $=0,015, \mathrm{p}<0,01)$, Tabakkonsum während der Schwangerschaft $(\mathrm{AME}=0,061, \mathrm{p}<0,01)$ sowie tendenziell auch Unter- $(\mathrm{AME}=0,011, \mathrm{p}=0,696)$ oder starkes Übergewicht $(\mathrm{AME}=0,013, \mathrm{p}=0,694)$ mit einem erhöhten Frühgeburtsrisiko verknüpft sind, die unregelmäßige sportliche Betätigung allerdings einen unerwartet, entgegengesetzten Einfluss auf die Frühgeburtsprävalenz ausübt (AME = -0,036, p < 0,10). ${ }^{45}$ Zudem erweisen sich die Vorsorgevariable sowie die anderen habituellen Merkmale (Risiko-

\footnotetext{
${ }^{45}$ Eine mögliche Erklärung für dieses Ergebnis ist, dass die hier betrachteten sportaktiven Frauen eher wehenfördernde Sportarten ausübten. Informationen zur Art der sportlichen Betätigung stellt das SOEP allerdings nicht bereit.
} 
bereitschaft, Kontrolle über eigenes Leben) als nahezu irrelevant für die Vorhersage einer Frühgeburt. Der Residualeffekt für die Gruppe der Hauptschulabsolventinnen nimmt im Vergleich von Modell 1 (AME =0,045, p < 0,10) zu Modell 4 (AME =0,034) zwar ab und ist mit $\mathrm{p}=0,179$ auch nicht mehr signifikant, allerdings ist dieses Erklärungspotenzial nicht dem Risikoverhalten, sondern fast ausschließlich der Kontrollüberzeugung, das eigene Leben sei von Glück abhängig, zuzuschreiben (Ergebnis einer hier nicht ausgewiesenen Modellspezifikation). ${ }^{46}$

Modell 5 führt schließlich die jeweils auf der Verhältnis-, Einstellungs- und Verhaltensebene angesiedelten Erklärungsstränge multivariat zusammen. Nach unserem Erklärungsmodell müsste sich der Schätzer für die unterste Bildungsstatusgruppe (Koeffizienten für Mütter) deutlich reduzieren. Dies ist mit Einschränkung der Fall. Erwartungsgemäß liegt der AME mit einem Wert von 0,017 zwar unter den Residualwerten aus den vorherigen Modellspezifikationen, ein Null-Effekt stellt sich jedoch nicht ein.

Zum Schluss wird ein sechstes Regressionsmodell geschätzt. Überprüft wird die Relevanz eines medizinischen Arguments für die Erklärung der Residualeffekte der weiblichen Bildung. Dieses besagt, dass beispielsweise Paritäten, untere Bildungsgruppen haben mehr Kinder, ein Erklärungsmechanismus für Bildungsunterschiede im Schwangerschaftsergebnis darstellen können (vgl. Bergmann und Dudenhausen 2003). Nach Aufnahme medizinisch relevanter (mit dem Bildungsabschluss von Schwangeren assoziierten) Einflussfaktoren der Frühgeburt (Parität, Alter zum Zeitpunkt der Schwangerschaft, Planung der Schwangerschaft, Gesundheitszustand im letzten Schwangerschaftsdrittel, Mehrlinge und Körpergröße), verändern sich die Koeffizienten für die unterste Bildungsgruppe (Modell 6) im Vergleich zum Vormodell (Modell 5) nur unwesentlich, sodass für die hier betrachteten Frauen ein über medizinische Pfade laufender Einfluss vermutlich auszuschließen ist.

\footnotetext{
${ }^{46}$ So liegt der AME unter ausschließlicher Berücksichtigung habitueller Variablen, wie bereits in Modell 4 ersichtlich, bei 0,034 ( $\mathrm{p}=0,137)$. Aufgrund des gegenläufigen Effekts der sportlichen Betätigung (höher gebildete Frauen sind häufiger sportlich aktiv, gleichzeitig wirkt eine regelmäßige sportliche Betätigung bei den hier betrachteten Frauen schwangerschaftsverkürzend) ist dieses Ergebnis jedoch mit Vorsicht zu betrachten, da in weiterführenden Analysen unter Ausschluss der sportlichen Betätigung das bereits vielfach bestätigte hohe Erklärungspotenzial des Tabakkonsums zum Tragen kommt.
} 
Tabelle 5.3: Logistische Regressionen zur Vorhersagewahrscheinlichkeit einer Frühgeburt (durchschnittlich marginale Effekte, multivariat)

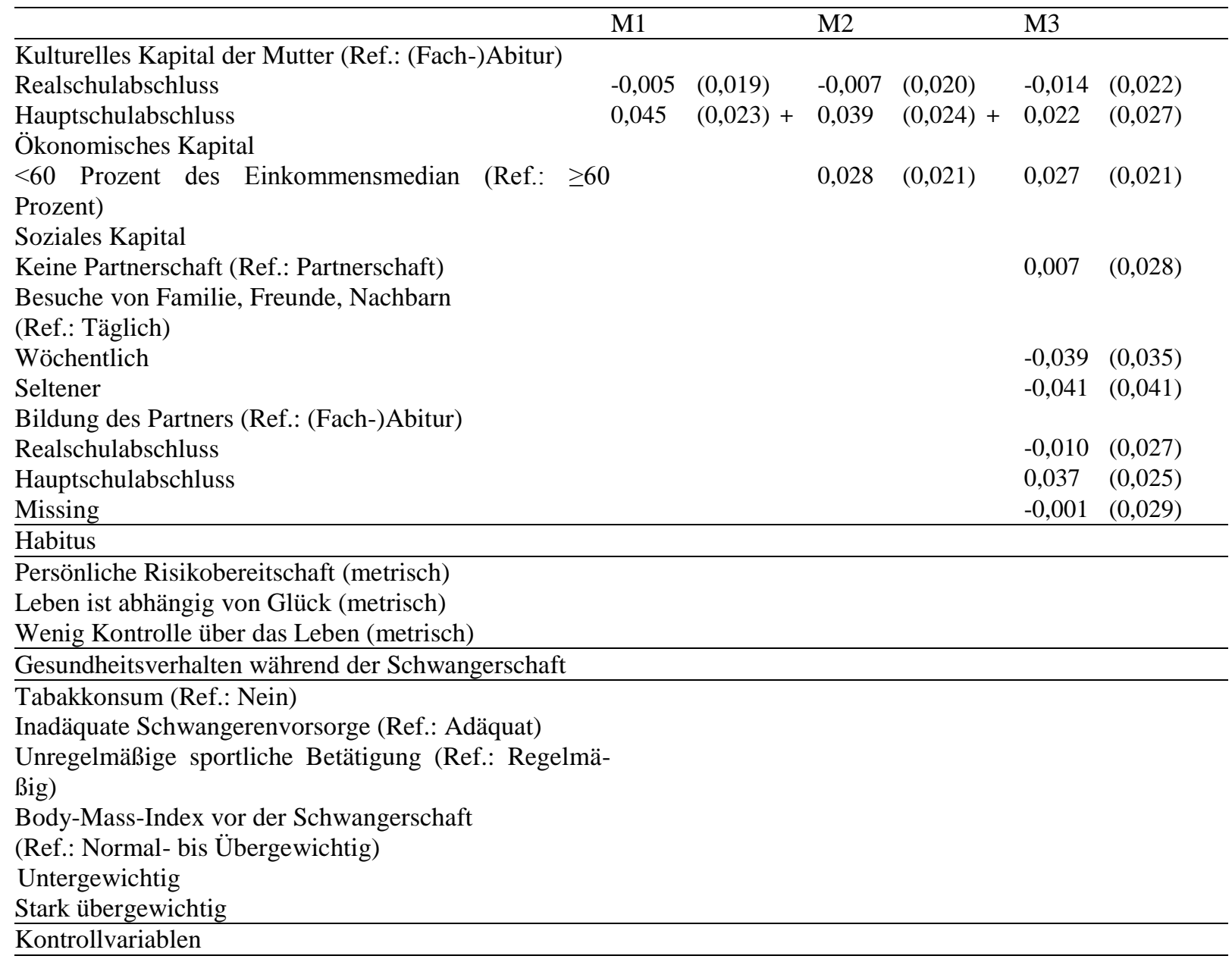

Mütterliches Alter vor der Schwangerschaft

(Ref.: 22 bis 34 Jahre)

$\leq 21$ Jahre

$\geq 35$ Jahre

Vorausgegangene Lebendgeburten

(Ref.: Zweitgebärende)

Erstgebärende

Mehrgebärende

Schwangerschaft geplant (Ref.: Ja)

Nein

Ja, mit medizinischer Unterstützung

Gesundheitszustand im letzten Schwangerschaftsdrittel

(Ref.: Sehr gut)

Gut

Zufriedenstellend/Schlecht

Mehrling

\begin{tabular}{llll}
\hline Durchschnittlich relativer Varianzanstieg (RVI) & 0,007 & 0,011 & 0,110 \\
Höchster Anteil fehlender Werte (FMI) & 0,025 & 0,047 & 0,423 \\
Beobachtungen & 1.316 & 1.316 & 1.316 \\
\hline
\end{tabular}

Quelle: SOEP 2003-2011; eigene Berechnungen, gerundet.

Anmerkungen: Robuste Standardfehler in Klammern; ***p<0.001; ** $\mathrm{p}<0.01 ; * \mathrm{p}<0.05 ;+\mathrm{p}<0.10$.

M1 - M6 unter Kontrolle von Migrationshintergrund der Mutter (Ref.: Nein), Ost-Deutschland (Ref.: West), Mädchen (Ref.: Junge), Geburtsjahr des Kindes (metrisch) und private Krankenversicherung (Ref.: Gesetzlich). 


\begin{tabular}{|c|c|c|c|c|c|c|}
\hline \multicolumn{7}{|l|}{ Tabelle 5.3 (Fortsetzung) } \\
\hline & M4 & & M5 & & M6 & \\
\hline \multicolumn{7}{|l|}{ Kulturelles Kapital der Mutter (Ref.: (Fach-)Abitur) } \\
\hline Realschulabschluss & $-0,012$ & $(0,021)$ & $-0,017$ & $(0,022)$ & $-0,017$ & $(0,022)$ \\
\hline Hauptschulabschluss & 0,034 & $(0,026)$ & 0,017 & $(0,028)$ & 0,019 & $(0,027)$ \\
\hline \multicolumn{7}{|l|}{ Ökonomisches Kapital } \\
\hline \multicolumn{7}{|l|}{ Prozent) } \\
\hline \multicolumn{7}{|l|}{ Soziales Kapital } \\
\hline Keine Partnerschaft (Ref.: Partnerschaft) & & & $-0,007$ & $(0,029)$ & $-0,019$ & $(0,029)$ \\
\hline \multicolumn{7}{|l|}{$\begin{array}{l}\text { Besuche von Familie, Freunde, Nachbarn } \\
\text { (Ref.: Täglich) }\end{array}$} \\
\hline Wöchentlich & & & $-0,038$ & $(0,036)$ & $-0,029$ & $(0,034)$ \\
\hline Seltener & & & $-0,037$ & $(0,043)$ & $-0,030$ & $(0,041)$ \\
\hline \multicolumn{7}{|l|}{ Bildung des Partners (Ref.: (Fach-)Abitur) } \\
\hline Realschulabschluss & & & $-0,014$ & $(0,027)$ & $-0,019$ & $(0,025)$ \\
\hline Hauptschulabschluss & & & 0,034 & $(0,025)$ & 0,031 & $(0,025)$ \\
\hline Missing & & & $-0,004$ & $(0,000)$ & $-0,005$ & $(0,029)$ \\
\hline \multicolumn{7}{|l|}{ Habitus } \\
\hline Persönliche Risikobereitschaft ( & 0,002 & $(0,004)$ & 0,002 & $(0,005)$ & 0,002 & $(0,005)$ \\
\hline Leben ist abhängig von Glück (metrisch) & 0,013 & $(0,005) * *$ & 0,013 & $(0,005) *$ & 0,013 & $(0,005) *$ \\
\hline Wenig Kontrolle über das Leben (metrisch) & $-0,003$ & $(0,006)$ & $-0,003$ & $(0,006)$ & $-0,003$ & $(0,006)$ \\
\hline \multicolumn{7}{|l|}{ Gesundheitsverhalten während der Schwangerschaft } \\
\hline Tabakkonsum (Ref.: Nein) & 0,055 & $(0,027) *$ & 0,051 & $(0,028)+$ & 0,051 & $(0,027)+$ \\
\hline Inadäquate Schwangerenvorsorge (Ref.: Adäquat) & $-0,011$ & $(0,023)$ & $-0,010$ & $(0,023)$ & $-0,004$ & $(0,022)$ \\
\hline $\begin{array}{l}\text { Unregelmäßige sportliche Betätigung (Ref.: Regelmä- } \\
\text { ßig) }\end{array}$ & i- $-0,055$ & $(0,020) * *$ & $-0,060$ & $(0,020) * *$ & $-0,060$ & $(0,019) * *$ \\
\hline \multicolumn{7}{|l|}{$\begin{array}{l}\text { Body-Mass-Index vor der Schwangerschaft } \\
\text { (Ref.: Normal- bis Übergewichtig) }\end{array}$} \\
\hline Untergewichtig & 0,006 & $(0,029)$ & 0,006 & $(0,029)$ & 0,005 & $(0,029)$ \\
\hline Stark übergewichtig & 0,008 & $(0,035)$ & 0,008 & $(0,035)$ & 0,002 & $(0,034)$ \\
\hline \multicolumn{7}{|l|}{ Kontrollvariablen } \\
\hline \multicolumn{7}{|l|}{$\begin{array}{l}\text { Mütterliches Alter vor der Schwangerschaft } \\
\text { (Ref.: } 22 \text { bis } 34 \text { Jahre) }\end{array}$} \\
\hline$\leq 21$ Jahre & & & & & $-0,061$ & $(0,035)+$ \\
\hline$\geq 35$ Jahre & & & & & 0,004 & $(0,021)$ \\
\hline \multicolumn{7}{|l|}{$\begin{array}{l}\text { Vorausgegangene Lebendgeburten } \\
\text { (Ref.: Zweitgebärende) }\end{array}$} \\
\hline Erstgebärende & & & & & 0,030 & $(0,019)$ \\
\hline \multirow{2}{*}{\multicolumn{7}{|c|}{ Schwangerschaft geplant (Ref.: Ja) }} \\
\hline & & & & & & \\
\hline Nein & & & & & 0,043 & $(0,019) *$ \\
\hline Ja, mit medizinischer Unterstützung & & & & & 0,131 & $(0,037) \stackrel{* *}{*}$ \\
\hline \multicolumn{7}{|l|}{$\begin{array}{l}\text { Gesundheitszustand im letzten Schwangerschaftsdrittel } \\
\text { (Ref.: Sehr gut) }\end{array}$} \\
\hline Gut & & & & & 0,073 & $(0,021) * * *$ \\
\hline Zufriedenstellend/Schlecht & & & & & 0,105 & $(0,028) * * *$ \\
\hline Mehrling & & & & & 0,066 & $(0,037)+$ \\
\hline Durchschnittlich relativer Varianzanstieg (RVI) & 0,311 & & 0,312 & & 0,243 & \\
\hline Höchster Anteil fehlender Werte (FMI) & 0,526 & & 0,542 & & 0,561 & \\
\hline Beobachtungen & 1.316 & & 1.316 & & 1.316 & \\
\hline
\end{tabular}

Quelle: SOEP 2003-2011; eigene Berechnungen, gerundet.

Anmerkungen: Robuste Standardfehler in Klammern; *** $<<0.001 ; * * \mathrm{p}<0.01 ; * \mathrm{p}<0.05 ;+\mathrm{p}<0.10$.

M1 - M6 unter Kontrolle von Migrationshintergrund der Mutter (Ref.: Nein), Ost-Deutschland (Ref.: West), Mädchen (Ref.: Junge), Geburtsjahr des Kindes (metrisch) und private Krankenversicherung (Ref.: Gesetzlich). 


\subsection{Diskussion und Forschungsausblick}

Am Beispiel der Frühgeburt wurde der Frage nachgegangen, inwiefern ökonomische und soziale Ressourcen sowie riskante Einstellungen und Verhaltensweisen das bildungsspezifische Frühgeburtsrisiko vermitteln. Wir nahmen an, dass variierende Frühgeburtsrisiken die Folge gesundheitsriskanter Wahrnehmungs- und Denkschemata (hohe persönliche Risikobereitschaft, geringe Kontrollüberzeugungen) und damit verknüpften Risikoverhaltensweisen (geringe Schwangerenvorsorge, Tabakkonsum, unregelmäßige sportliche Betätigung und BodyMass-Index) sind. Aufgrund der engen Wechselbeziehung zum Habitus, sind wir von einer zentralen ungleichheitsverursachenden Wirkung des Kulturkapitals ausgegangen, während wir beim ökonomischen und sozialen Kapital neben direkten Effekten auch eine den Bildungseinfluss auf die Frühgeburt vermittelnde Wirkung erwarteten. Die empirische Überprüfung unseres Erklärungsmodells basiert auf den Daten des Sozio-oekonomischen Panels (SOEP) der Wellen 2003 bis 2011.

Wie die Befunde multipler Regressionsanalysen zeigen, lässt sich eine bedeutsame Rolle des geringen mütterlichen kulturellen Kapitals für die Startchancen von Neugeborenen feststellen. Dieser Zusammenhang lässt sich jedoch nur teilweise durch die simultane Berücksichtigung von Verhältnis-, Einstellungs- und Verhaltensindikatoren aufklären. Auch finden nicht alle kapitaltheoretisch begründeten Hypothesen empirische Bestätigung.

Erwartungsgemäß geht vom ökonomischen Kapital (weniger als $60 \%$ des Einkommensmedian) ein direkter Effekt auf die Wahrscheinlichkeit einer Frühgeburt aus. Wie für die elterliche Bildung, zeigt sich jedoch kein linearer Zusammenhang, sondern eher ein Schwellenwert-Effekt (Blumenshine et al. 2010). Auch lässt sich eine (schwache) vermittelnde Wirkung des Armutsindikators auf die Bildungsunterschiede im Frühgeburtsrisiko beobachten. Dies steht im Einklang mit der Überlegung, dass ökonomisches Kapital insbesondere für gering Gebildete von gesundheitlicher Bedeutung ist (vgl. Mirowsky und Ross 2003, 2007). Keine Bestätigung finden unsere Annahmen bezüglich der Relevanz der Sozialkapitalaspekte Anwesenheit eines Partners und regelmäßige soziale Kontakte. Lediglich für das (gering ausgeprägte) Bildungskapital des Partners beobachten wir einen über alle Modelle hinweg stabilen, aber insignifikanten Frühgeburtseffekt, was tendenziell auf die negative Seite sozialer Eingebundenheit (bspw. Verstärkung gesundheitsriskanten Verhaltens der Mutter durch das geringe gesundheitsrelevante Kulturkapital des Partners) verweist (vgl. Shah et al. 2011).

Im Hinblick auf das soziale Kapital besteht möglicherweise ein Validitätsproblem. Zwar stellt das Bildungskapital des Partners wertvolles gesundheitsrelevantes Sozialkapital dar, allerdings ist Bourdieus Sozialkapitaldefinition sehr unspezifisch (vgl. Haug 1997). Damit ist fraglich, inwiefern unsere Variablen zum Sozialkapital auch wirklich das Sozialkapital schwangerer Frauen messen. Schließlich dürfte die Gesamtheit des Beziehungskapitals erst durch eine Bündelung quantitativer und qualitativer Aspekte ausreichend abgedeckt sein, deren operationale Konkretisierung wegen weitestgehender Uneinigkeit darüber, was Sozialkapital überhaupt ist und wie es gemessen werden kann (vgl. Franzen und Freitag 2007), 
schwierig und nur theoriegeleitet vorzunehmen ist. Insbesondere qualitative Formen sozialer Eingebundenheit konnten bis auf den relativ groben Indikator für das gesundheitskulturelle Kapital des Partners (formale Bildung) nur unzureichend berücksichtigt werden. Auch findet sich in der Partnerschaftsvariable wenig Varianz, d. h. die Mehrheit der Frauen (>90 \%) hat während der Schwangerschaft einen Partner an ihrer Seite, woraus für alle Bildungsgruppen geringe Zellbesetzungen resultieren. Angesichts der ebenfalls geringen Zahl von zu früh Geborenen kann statistisch nicht ausgeschlossen werden, dass wir mit der Ablehnung unserer Sozialkapitalhypothesen einen Irrtum begehen.

Im Zusammenhang mit der geringen Stichprobengröße, geringer Beobachtungen der zu früh Geborenen und der relativ geringen Erklärungskraft unserer Regressionsmodelle wurde auf die empirische Prüfung von (aus kapitaltheoretischer Sicht interessanten) wechselseitigen Beziehungen zwischen den Kapitalformen verzichtet. Beispielsweise ist denkbar, dass insbesondere Frauen mit dem geringsten kulturellen Kapital von einer hohen Qualität sozialen Kapitals (relativ höhere Bildung des Partners) oder einer materiellen Besserstellung gesundheitlich profitieren. Gesundheitsvorteile sind deshalb wahrscheinlicher, da bei Frauen durch Hinzugewinne ökonomischen Kapitals eine Vertikalverlagerung nach oben stattfindet. Dennoch verweist eine skeptische Sicht, nach der Maxime „It takes a great deal of money to compensate for low education“ (Mirowsky und Ross 2003: 196) auf die Beharrungs- und Strukturierungskraft gesundheitlicher Grundorientierungen. Analog verhält es sich mit dem qualitativen Sozialkapitalaspekt. Kommt es dauerhaft, wie typischerweise in bildungsheterophilen Partnerschaften, zu einer Überschneidung habitueller Grundeinstellungen (bringt der Partner also mehr gesundheitskulturelles Kapital in den Haushalt ein), könnten sich gesundheitsbezogene informelle Lern- und Kontrollprozesse (vgl. Mirowsky und Ross 2003: 196) positiv auf das Gesundheitshandeln von Schwangeren auswirken und damit einhergehend das Risiko von Schwangerschaftskomplikationen und Frühgeburten senken. Weiterführende, hier nicht dargestellte Analysen bestätigen diese möglichen Kapitalinterdependenzen jedoch nicht. Letzteres ist aufgrund geringer Zellbesetzungen aber nur mit Vorsicht zu interpretieren.

Eine Stärke unseres Beitrags liegt darin, dass wir mit den verwendeten Daten (zumindest) näherungsweise den Habitus operationalisieren. Dennoch muss einschränkend hinzugefügt werden, dass es sich bei den hier verwendeten Wahrnehmungs- und Denkschemata um sehr grobe Indikatoren handelt, die nicht zwangsläufig mit gesundheitlichen Aspekten im Zusammenhang stehen müssen. Wahrnehmungsschemata sind lediglich über die allgemeine persönliche Risikobereitschaft abgebildet. Ob dieses Maß die theoretisch begründete gesundheitsbezogene Risikowahrnehmung tatsächlich widerspiegelt, ist fraglich. Weiter sind die Denkschemata nur anhand zweier Kontrollüberzeugungen (Leben ist abhängig von Schicksal und Glück; wenig Kontrolle über das Leben) operationalisiert. Eine Beständigkeit verinnerlichter Einstellungen vorausgesetzt, haben wir (aufgrund unzureichender Datenlage) die ausschließlich in den Jahren 2005 und 2010 erfassten Denkschemata auf die anderen sieben Befragungsjahre übertragen. Zwar findet unsere Annahme, dass mit abnehmenden Bildungsgrad geringere Kontrollüberzeugungen einhergehen, Bestätigung. Jedoch birgt die über alle Modelle hin- 
weg konstant starke Erklärungskraft der Kontrollüberzeugung, das eigene Leben wäre von Schicksal und Glück abhängig, ein Endogenitätsproblem. So ist denkbar, dass diese Einstellung infolge einer früheren vorzeitigen Entbindung geprägt ist. Schließlich konnten wir Verhaltensintentionen (Handlungsschemata) nicht abbilden. Es wird also ein theoretischer Mechanismus unterstellt, obwohl der Zusammenhang zwischen Intention und tatsächlich ausgeführtem Gesundheitsverhalten auch über andere Einflusskanäle (z. B. Stress) erfolgen kann (vgl. Lampert und Ziese 2005). Diese finden in den Analysen aber nur teilweise Berücksichtigung (Proxys: Gesundheit im letzten Schwangerschaftsdrittel; ungeplante Schwangerschaft).

Im Hinblick auf die Erklärungskraft des Gesundheitsverhaltens während der Schwangerschaft (Schwangerenvorsorge, Tabakkonsum, sportliche Betätigung, Body-Mass-Index) finden unsere Annahmen nur für den Tabakkonsum Bestätigung. So erklärt das Rauchverhalten (in gesonderten Analysen, die nicht den Sportindikator enthalten) einen Teil des höheren Frühgeburtsrisikos von Hauptschulabsolventinnen. Dieser Befund steht im Einklang mit dem Forschungsstand, wie beispielsweise mit der von Currie und Moretti (2003) für die USamerikanische Bevölkerung durchgeführte Studie, in der eine (durch Veränderungen der Bildungsinfrastruktur herbeigeführte) Erhöhung des weiblichen Bildungskapitals zur Senkung der Frühgeburtsprävalenz beitrug. Sowohl die Vorsorgevariable von Currie und Moretti (Teilnahme an der Schwangerenvorsorge im ersten Trimester) als auch unsere (ärztliche Inanspruchnahme in Verbindung mit dem Schwangerschaftsmonat) stellen jedoch keine verzerrungsfreien Vorsorgeindikatoren dar. Ferner werfen diese Maße ebenfalls ein Endogenitätsproblem auf. So ist zu hinterfragen, ob Frauen aufgrund der Relevanz pränataler Diagnostik oder aufgrund gesundheitlicher Probleme an der Schwangerenvorsorge teilnehmen. Zwar kontrollierten wir deshalb auch für den Gesundheitszustand im letzten Schwangerschaftsdrittel und in Robustheitsanalysen auch für die Gesundheit vor der Schwangerschaft (was die Ergebnisse nicht veränderte), wertvolle objektive Gesundheitskriterien stellen diese subjektiven Selbsteinschätzungen jedoch nicht dar. Zudem ist unsere Vorsorgevariable hinsichtlich der Konzipierung aus ärztlicher Inanspruchnahme in Verbindung mit dem Schwangerschaftsmonat nicht verzerrungsfrei. So muss die Anzahl der Arztbesuche nicht zwangsläufig mit dem Vorsorgeverhalten in Verbindung stehen. Dies könnte auch erklären, warum wir, entgegen der von Simoes und Kollegen (2003) durchgeführten Studie mit deutschen Perinataldaten, die mit ca. 92 bis $99 \%$ erfassten Entbindungen ein nahezu realitätsgetreues Abbild bieten (BQS 2012; Günter et al. 2007; Simoes et al. 2003; Reime et al. 2006; Koller et al. 2008), bereits einen gegen null gehenden, insignifikanten Bruttoeffekt haben.

Unwahrscheinlich ist ein insignifikanter Effekt des Vorsorgehandelns aber nicht, wie internationale Studien belegen (Reime et al. 2006). Eine weitere methodische Schwierigkeit stellt die hohe Anzahl fehlender Werte in den zentralen Modellvariablen dar (Erfassung der Kapitalien während der Schwangerschaft, der inkorporierten Wahrnehmungs- und Denkschemata und des Risikoverhaltens). So werden Fragen zum Gesundheitsverhalten nicht jedes Jahr erfasst (vgl. Peters 2010), was insbesondere beim Ernährungsverhalten und Alkoholkonsum zu hohen fehlenden Werten (> $70 \%$ ) und damit einhergehend zum Ausschluss dieser Variablen 
führte. Um fehlende Werte niedrig zu halten, wurden jene Frauen, bei denen keine Befragung im Zeitraum der Schwangerschaft stattfand, nicht in die Analysen einbezogen und fehlende Angaben mit dem Schätzverfahren der multiplen Imputation durch plausible Werte ersetzt. Allerdings vertritt Spieß (2010: 128) die Ansicht, dass ,[b]ei einem [Missing-]Anteil von mehr als $50 \%$ (...) den Imputationsmodellen ein zu hohes Gewicht zu[kommt]“. In unseren Analysen sind die Variablen ,persönliche Risikobereitschaft“, „Tabakkonsum“ und „BodyMass-Index“ mit mehr als $50 \%$ fehlenden Werten vertreten. Zwar wurde der Schätzprozess 100-mal wiederholt, eine Ergebnisverzerrung aufgrund der angewendeten Imputationen bei sehr hohen prozentualen Ausfällen ist allerdings nicht auszuschließen.

Folgende Kriterien sprechen jedoch für die Güte der durch das Verfahren der multiplen Imputation gewonnenen Schätzergebnisse: So liegt der über alle Koeffizienten berechnete durchschnittlich relative Varianzanstieg (RVI) maximal bei 0,31 (siehe Modell 4, Tabelle 5.3; vgl. StataCorp 2009: 48), die höchste empfohlene Imputationszahl bei 56 (siehe Modell 6 (100*FMI), Tabelle 5.3; StataCorp 2009: 48) und der berechnete „Monte Carlo Error“ (nicht in Tabelle ausgewiesen) bei allen im Endmodell ausgewiesen Variablen (ausgenommen: Besuchshäufigkeit, persönlicher Risikobereitschaft, BMI) bei p < 0,01 bzw. p < 0,02 (StataCorp 2009; White et al. 2011: 388). Zudem zeigten Robustheitsanalysen unter der Anwendung des fallweisen Ausschlusses keine nennenswerten Unterschiede der multivariaten Ergebnisse.

Zusammenfassend kann der Bildungseinfluss durch das in der vorliegenden Arbeit verwendete Variablenset nur teilweise aufgeklärt werden. Die (insignifikanten) Koeffizienten dürften auf nicht-beobachtete Drittvariablen, inadäquate Messungen der theoretischen Konzepte (bspw. gesundheitsbezogenen Grundorientierungen oder der selbst konzipierten Variable zur Schwangerenvorsorge) oder auf gegenläufige Assoziationen mit der abhängigen Variable (Besuchshäufigkeit, sportliche Betätigung) zurückzuführen sein. Angesichts bedeutsamer Residualeffekte der elterlichen Bildung auf die Frühgeburt, insbesondere mit Blick auf intervenierende Größen wie dem gesundheitsbezogenen Habitus (vgl. Sperlich und Mielck 2003) oder der Schwangerenvorsorge, besteht weiterhin ein großer Forschungsbedarf. 


\section{Anhang}

Tabelle A 5.1: Verteilung der Kontrollvariablen nach mütterlichem Kulturkapital

\begin{tabular}{|c|c|c|c|c|c|c|}
\hline & \multicolumn{2}{|c|}{ Hauptschulabschluss } & \multicolumn{2}{|c|}{ Realschulabschluss } & \multicolumn{2}{|c|}{ (Fach-)Abitur } \\
\hline & Prozent & (Anzahl) & Prozent & (Anzahl) & Prozent & (Anzahl) \\
\hline \multicolumn{7}{|c|}{ Migrationshintergrund der Mutter*** } \\
\hline Nein & 58,26 & $(141)$ & 81,13 & $(417)$ & 83,75 & $(469)$ \\
\hline Direkt & 24,79 & $(60)$ & 12,26 & $(63)$ & 9,46 & $(53)$ \\
\hline Indirekt & 16,94 & (41) & 6,61 & $(34)$ & 6,79 & $(38)$ \\
\hline \multicolumn{7}{|l|}{ Region*** } \\
\hline West & 87,60 & $(212)$ & 70,62 & $(363)$ & 73,75 & $(413)$ \\
\hline Ost & 12,40 & $(30)$ & 29,38 & $(151)$ & 26,25 & $(147)$ \\
\hline \multicolumn{7}{|l|}{ Krankenversicherung $* * *$} \\
\hline Gesetzlich & 99,17 & $(240)$ & 95,91 & $(493)$ & 78,39 & $(439)$ \\
\hline Privat & 0,83 & (2) & 4,09 & $(21)$ & 21,61 & $(121)$ \\
\hline \multicolumn{7}{|l|}{ Geschlecht des Kindes } \\
\hline Junge & 54,96 & $(133)$ & 51,56 & $(265)$ & 50,71 & $(284)$ \\
\hline Mädchen & 45,04 & (109) & 48,44 & $(249)$ & 49,29 & $(276)$ \\
\hline \multicolumn{7}{|c|}{$\begin{array}{l}\text { Mütterliches Alter vor der Schwanger- } \\
\text { schaft*** }\end{array}$} \\
\hline$\leq 21$ Jahre & 16,12 & $(39)$ & 7,20 & $(37)$ & 1,43 & $(8)$ \\
\hline$\geq 35$ Jahre & 13,64 & $(33)$ & 16,73 & $(86)$ & 23,75 & (133) \\
\hline 22 bis 34 Jahre & 70,25 & $(170)$ & 76,07 & $(391)$ & 74,82 & $(419)$ \\
\hline \multicolumn{7}{|l|}{ Muttergesundheit im letzten } \\
\hline \multicolumn{7}{|l|}{ Schwangerschaftsdrittel $* * *$} \\
\hline Sehr gut & 24,38 & $(59)$ & 29,57 & $(152)$ & 38,39 & $(215)$ \\
\hline Gut & 58,26 & $(141)$ & 61,87 & $(318)$ & 55,18 & $(309)$ \\
\hline Zufriedenstellend/Schlecht & 17,36 & $(42)$ & 8,56 & $(44)$ & 6,43 & $(36)$ \\
\hline \multicolumn{7}{|l|}{ Schwangerschaft geplant $* * *$} \\
\hline Nein & 37,60 & $(91)$ & 25,29 & $(130)$ & 18,21 & $(102)$ \\
\hline $\mathrm{Ja}$ & 61,57 & (149) & 72,96 & $(375)$ & 78,39 & $(439)$ \\
\hline Ja, mit medizin. Unterstützung & 0,83 & $(2)$ & 1,75 & $(9)$ & 3,39 & $(19)$ \\
\hline \multicolumn{7}{|l|}{ Mehrling } \\
\hline $\mathrm{Ja}$ & 1,24 & (3) & 1,56 & $(8)$ & 1,96 & $(11)$ \\
\hline Nein & 98,76 & $(239)$ & 98,44 & $(506)$ & 98,04 & $(549)$ \\
\hline \multicolumn{7}{|l|}{ Parität*** } \\
\hline Erstgebärende & 32,32 & (78) & 43,00 & $(221)$ & 47,86 & $(268)$ \\
\hline Zweitgebärende & 37,19 & (90) & 39,11 & $(201)$ & 37,50 & (210) \\
\hline Mehrgebärende & 30,58 & (74) & 17,90 & $(92)$ & 14,64 & $(82)$ \\
\hline $\mathrm{N}$ & & $(242)$ & & $(514)$ & & $(560)$ \\
\hline
\end{tabular}


Tabelle A 5.2: $\quad$ Logistische Regressionen zur Vorhersagewahrscheinlichkeit einer Frühgeburt (univariat)

\begin{tabular}{|c|c|c|c|}
\hline & AME & S.E & \\
\hline \multicolumn{4}{|l|}{ Kulturelles Kapital der Mutter (Ref.: (Fach-)Abitur) } \\
\hline Realschulabschluss & $-0,006$ & $(0,019)$ & \\
\hline Hauptschulabschluss & 0,047 & $(0,021)$ & $*$ \\
\hline \multicolumn{4}{|l|}{ Ökonomisches Kapital } \\
\hline$<60$ Prozent des Einkommensmedian (Ref.: $\geq 60$ Prozent) & 0,034 & $(0,020)$ & + \\
\hline \multicolumn{4}{|l|}{ Soziales Kapital } \\
\hline Keine Partnerschaft (Ref.: Partnerschaft) & 0,008 & $(0,026)$ & \\
\hline \multicolumn{4}{|l|}{ Besuche von Familie, Freunde, Nachbarn (Ref.: Täglich) } \\
\hline Wöchentlich & $-0,047$ & $(0,034)$ & \\
\hline Seltener & $-0,053$ & $(0,039)$ & \\
\hline \multicolumn{4}{|l|}{ Bildung des Partners (Ref.: (Fach-)Abitur) } \\
\hline Realschulabschluss & $-0,013$ & $(0,026)$ & \\
\hline Hauptschulabschluss & 0,046 & $(0,021)$ & $*$ \\
\hline Missing & $-0,002$ & $(0,026)$ & \\
\hline \multicolumn{4}{|l|}{ Habitus } \\
\hline Persönliche Risikobereitschaft (metrisch) & 0,003 & $(0,005)$ & \\
\hline Leben ist abhängig von Glück (metrisch) & 0,015 & $(0,005)$ & $* *$ \\
\hline Wenig Kontrolle über das Leben (metrisch) & 0,002 & $(0,005)$ & \\
\hline \multicolumn{4}{|l|}{ Gesundheitsverhalten während der Schwangerschaft } \\
\hline Tabakkonsum (Ref.: Nein) & 0,061 & $(0,023)$ & $* *$ \\
\hline Inadäquate Schwangerenvorsorge (Ref.: Adäquat) & $-0,004$ & $(0,022)$ & \\
\hline Unregelmäßige sportliche Betätigung (Ref.: Regelmäßig) & $-0,036$ & $(0,018)$ & + \\
\hline \multicolumn{4}{|l|}{ Body-Mass-Index vor der Schwangerschaft (Ref.: Normal- bis Übergewichtig) } \\
\hline Untergewichtig & 0,011 & $(0,029)$ & \\
\hline Stark übergewichtig & 0,013 & $(0,033)$ & \\
\hline \multicolumn{4}{|l|}{ Migrationshintergrund der Mutter (Ref.: Nein) } \\
\hline Direkter Migrationshintergrund & 0,033 & $(0,021)$ & \\
\hline Indirekter Migrationshintergrund & 0,014 & $(0,027)$ & \\
\hline Ost-Deutschland (Ref.: West) & $-0,013$ & $(0,019)$ & \\
\hline Mädchen (Ref.: Junge) & $-0,007$ & $(0,016)$ & \\
\hline Geburtsjahr des Kindes (metrisch) & $-0,008$ & $(0,003)$ & $*$ \\
\hline Private Krankenversicherung (Ref.: Gesetzlich) & 0,016 & $(0,028)$ & \\
\hline \multicolumn{4}{|l|}{ Mütterliches Alter vor der Schwangerschaft (Ref.: 22 bis 34 Jahre) } \\
\hline$\leq 21$ Jahre & 0,004 & $(0,031)$ & \\
\hline$\geq 35$ Jahre & $-0,012$ & $(0,022)$ & \\
\hline \multicolumn{4}{|l|}{ Vorausgegangene Lebendgeburten (Ref.: Zweitgebärende) } \\
\hline Erstgebärende & 0,031 & $(0,017)$ & + \\
\hline Mehrgebärende & 0,012 & $(0,023)$ & \\
\hline \multicolumn{4}{|l|}{ Schwangerschaft geplant (Ref.: Ja) } \\
\hline Nein & 0,048 & $(0,017)$ & $* *$ \\
\hline Ja, mit medizinischer Unterstützung & 0,126 & $(0,041)$ & $* *$ \\
\hline \multicolumn{4}{|l|}{ Gesundheitszustand im letzten Schwangerschaftsdrittel (Ref.: Sehr gut) } \\
\hline Gut & 0,069 & $(0,022)$ & $* *$ \\
\hline Zufriedenstellend/Schlecht & 0,119 & $(0,028)$ & $* * *$ \\
\hline Mehrling (Ref.: Nein) & 0,111 & $(0,039)$ & $* *$ \\
\hline Beobachtungen & 1.316 & & \\
\hline
\end{tabular}

Quelle: SOEP 2003-2011; eigene Berechnungen, gerundet.

Anmerkungen: Robuste Standardfehler in Klammern; ***p<0.001; ** $\mathrm{p}<0.01 ; * \mathrm{p}<0.05 ;+\mathrm{p}<0.10$. 


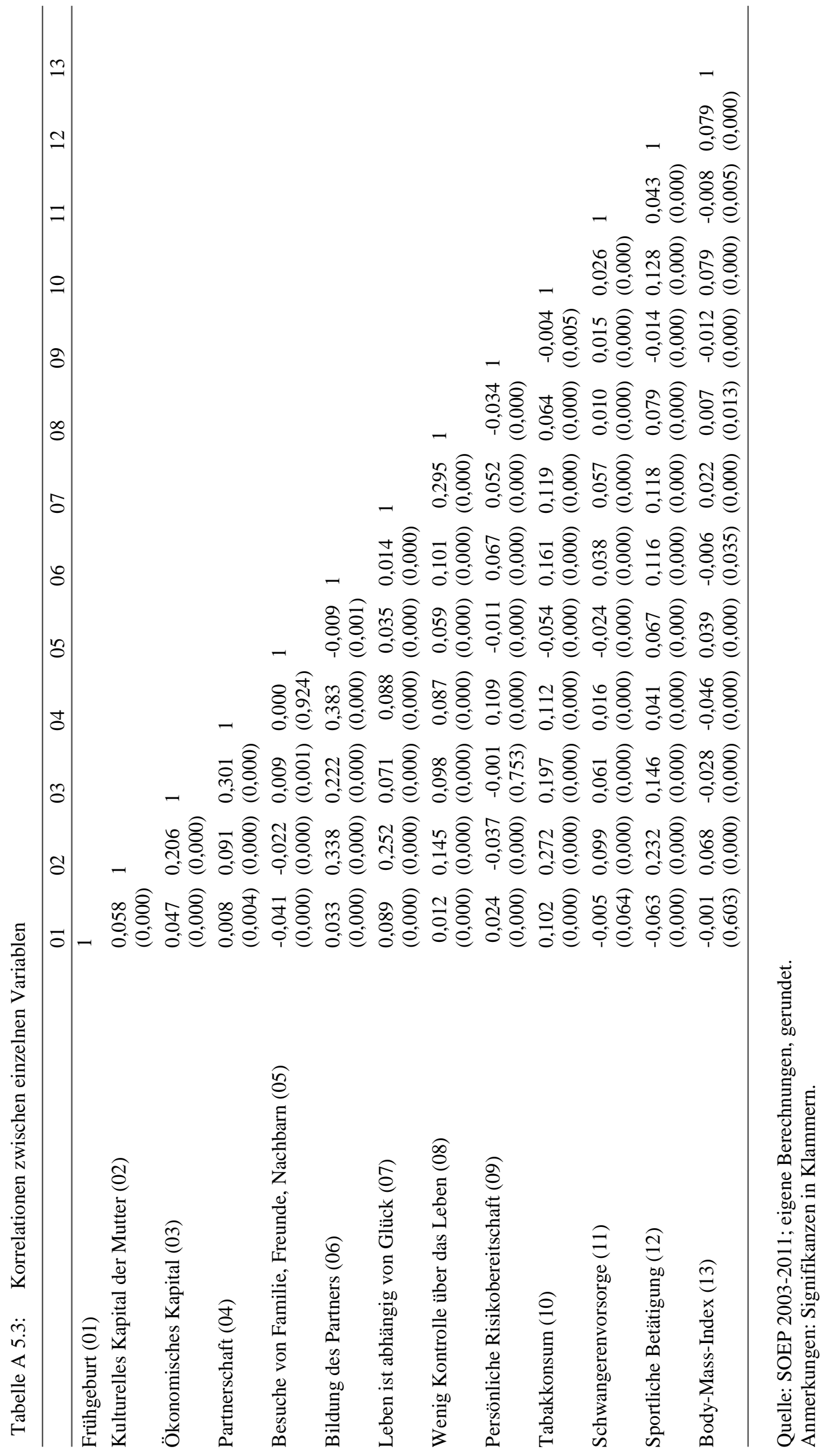




\section{Gesundheitliche Ungleichheit im Vorsorgestatus Neugeborener}

\section{1 $\quad$ Einleitung $^{47}$}

Aus soziologischer Sicht stellen Gesundheit und Krankheit primär soziale Phänomene dar (Faltermaier et al. 1998: 12; House et al. 1988: 294; Hurrelmann 1988: 17; Wendt und Wolf 2006: 16). Als empirisch gesichert gilt die Wirksamkeit sozialer Einflussgrößen auf die Gesundheitschancen. Bereits die Kennziffern zur Lebensfähigkeit von Neugeborenen - wie das Geburtsgewicht und das Säuglingssterblichkeitsrisiko - variieren nach sozialen Hintergrundmerkmalen der Mutter (Jungbauer-Gans und Kriwy 2004: 10f.). Auch beim Eintritt in das Bildungssystem lassen sich systematische soziale Disparitäten in der kognitiven und gesundheitlichen Entwicklung feststellen (Bettge et al. 2007: 45; Biedinger und Becker 2006). Schlechte gesundheitliche Grundlagen im Kindesalter setzen sich oft in Gesundheitsstörungen und Krankheitsrisiken im Jugend- und Erwachsenenalter fort (Dragano und Siegrist 2009; Lampert und Richter 2006: 209; Lampert et al. 2005: 97; Richter und Hurrelmann 2007: 8). ${ }^{48}$ Da die gesundheitlichen Weichen für den weiteren Lebensverlauf schon in frühem Lebensalter gestellt werden (Richter und Hurrelmann 2016), ist die Altersgruppe der Neugeborenen bis unter Dreijährigen von besonderem Interesse für gesundheitsbezogene Ungleichheitsanalysen.

Die Identifizierung der Ungleichheitsmechanismen gilt als unabdingbar für die Entwicklung intervenierender Maßnahmen zur Verringerung des sozialen Gesundheitsgefälles: „Nur wer die Ursachen kennt, kann eingreifen und verbessern.“ (Hradil 2006: 33). Allerdings steht eine Offenlegung der Bestimmungsgründe gesundheitlicher Ungleichheiten nach wie vor weitgehend aus, weshalb nur unzureichend geklärt ist, warum Menschen in Abhängigkeit ihrer sozialen Position unterschiedlich gesund sind und unterschiedlich gesundheitlich handeln.

In der vorliegenden Arbeit liegt daher das Hauptaugenmerk auf den Mechanismen, die hinter dem sozialen Gesundheitsgefälle im Kindesalter stehen. Im Anschluss an Jungbauer-Gans (2002: 37f.) wird angenommen, dass Gesundheit und hierauf bezogenes Handeln im Alltag in erster Linie durch drei Ressourcenbündel beeinflusst wird: Materielle Ressourcen (durch weniger belastende und bessere Lebens- und Arbeitsbedingungen), persönliche Ressourcen (Intelligenz, Bildung, Copingkompetenzen) sowie soziale Ressourcen (soziale Unterstützung).

In diesem Beitrag liegt der Fokus auf dem Einfluss sozialer Integrations- und Unterstützungsstrukturen der Mutter auf die Inanspruchnahme von Vorsorgeuntersuchungen für Kinder. Im Detail wird untersucht, ob schichtspezifische Unterschiede im Besuch von Vorsorgeuntersuchungen für Säuglinge und Kinder bis 18 Monate über eine mehr oder weniger gute Einbindung in soziale Beziehungen erklärt werden kann.

\footnotetext{
${ }^{47}$ Bei diesem Kapitel handelt es sich um die ausführlichere und überarbeitete Fassung eines Artikels, der im Jahr 2011 in Schmollers Jahrbuch unter dem Titel „Social Inequality in Early Childhood Health. Participation in the Preventive Health Care Program for Children" (Becker und Kurz 2011) erschien.

${ }^{48}$ So steht beispielsweise ein niedriges Geburtsgewicht in einem signifikanten Zusammenhang mit einem erhöhten Risiko frühzeitig im Erwachsenenalter zu versterben (Dragano und Siegrist 2006: 17f.).
} 
Für die Bundesrepublik liegen bislang nur wenige Studien zum Zusammenhang von Gesundheitsverhalten und Sozialkapital vor. Diese sprechen jedoch für das Erklärungspotential des Sozialkapitalkonzepts für Gesundheit und Gesundheitsverhalten im Kindes-, Jugend- und Erwachsenenalter (Jungbauer-Gans 2002; Klocke und Becker 2003; Lampert und Kroll 2006; Richter 2005).

Für die Analyse des Gesundheitsverhaltens in der frühen Kindheit ${ }^{49}$ stellt das Soziooekonomische Panel (SOEP) eine umfassende, aktuelle und bundesweit repräsentative Datengrundlage dar. Der erstmalig 2003 eingesetzte Fragebogen „Mutter und Kind“ stellt mit 19 Fragen zu Schwangerschaft, Kind- und Muttergesundheit, gesundheitsbezogenem Handeln sowie zur sozialen Ressourcenausstattung geeignete Daten für die Analyse gesundheitlicher Ungleichheiten zur Verfügung. Die Bedeutsamkeit sozialen Kapitals für die Neugeborenengesundheit untersuchen wir anhand der Frage zur Teilnahme an den Vorsorgeuntersuchungen für Säuglinge. Die Teilnahme an präventiven Maßnahmen gilt als eine wichtige Determinante für das Entstehen gesundheitlicher Ungleichheit (Jungbauer-Gans und Kriwy 2004: 15; Mueller und Heinzel-Gutenbrunner 2001: 9). In Deutschland gibt es seit 1971 ein zehn Untersuchungen umfassendes kostenloses Krankheitsfrüherkennungsprogramm für Kinder (Lampert et al. 2005: 104; Meyer-Nürnberger 2002: 862). Für das Kindesalter werden neun Untersuchungen (U1-U9) und zwischen dem 11. und 15. Lebensjahr eine weitere Untersuchung (J1) angeboten. Die Teilnahme am Präventionsprogramm ist freiwillig. Einschulungsuntersuchungen belegen eine seltenere Inanspruchnahme von Kindervorsorgeuntersuchungen in den unteren Sozialschichten (Lampert et al. 2005: 105). Die verwendete Datenbasis ermöglicht es, Rückschlüsse über die Teilnahme an den ersten Vorsorgeuntersuchungen sowohl in Abhängigkeit des sozialen Hintergrunds als auch der Sozialkapitalausstattung zu ziehen.

Im Weiteren wird wie folgt vorgegangen: Zunächst werden die klassischen Erklärungsansätze aus der Gesundheitsforschung und die an ihnen geübte Kritik vorgestellt (Abschnitt 6.2). Sodann wird in das Sozialkapitalkonzept als neuen und vielversprechenden Erklärungsansatz für das Phänomen gesundheitlicher Ungleichheiten eingeführt (Abschnitt 6.3). Es folgt die Darstellung der Hypothesen und ihre theoretische Einbindung (Abschnitt 6.4). Im empirischen Teil werden Datenbasis und Variablenkonstruktion (Abschnitt 6.5) sowie die Ergebnisse präsentiert (Abschnitt 6.6). Mit der Ergebnisdiskussion schließt der Beitrag (Abschnitt 6.7).

\subsection{Zur Erklärung gesundheitlicher Ungleichheit}

Bei der Erklärung gesundheitlicher Ungleichheit stützt sich die Gesundheitsforschung auf zwei Haupthypothesen (Richter 2005: 139; Richter und Hurrellmann 2006: 18): die Selektionshypothese, die soziale Aufstiege von Gesunden und Abstiege von Kranken postuliert, sowie die Verursachungshypothese, die annimmt, dass sich soziale Ungleichheit über verhal-

\footnotetext{
${ }^{49}$ Die erste Lebensphase nach der Geburt bis zum Eintritt in das Kindergartenalter wird als frühe Kindheit bezeichnet. Diese lässt sich differenzieren in das Säuglingsalter (0-1 Jahr) und das Kleinkindalter (2-3 Jahre) (Bründel und Hurrelmann 1996: 25).
} 
tensbezogene (Tabak- und Alkoholkonsum, Ernährung, Bewegung, Gesundheitsvorsorge) und strukturelle Faktoren (Einkommens-, Wohn- und Arbeitsverhältnisse) negativ auf die Gesundheit auswirkt. ${ }^{50}$ Hierbei wird sozialstrukturellen Ursachen ein größerer absoluter Erklärungsbeitrag im Vergleich zu verhaltensbezogenen Einflüssen zugeschrieben (Richter 2005: 140f.). Die Mehrheit der Studien zu Kindes- und Jugendalter geht von der Verursachungshypothese aus. Diese ist aus empirischer Sicht der konkurrierenden Selektionshypothese ${ }^{51}$ überlegen (Jungbauer-Gans und Kriwy 2004: 14; Richter und Hurrelmann 2006: 18). So spricht beispielsweise die Wirkung des sozialen Hintergrunds auf die Entwicklung im Mutterleib sowie auf die Kindersterblichkeit - selbst dann, wenn in den Analysen die pränatale Entwicklung statistisch kontrolliert wird - klar für die Verursachungshypothese (Dragano und Siegrist 2009).

Für die Fokussierung auf sozialstrukturelle oder verhaltensgebundene Einflüsse wird die Gesundheitsforschung jedoch stark kritisiert. Das Ausblenden von kulturellen und sozialen Ressourcen (Abel et al. 2009; Richter 2005) brachte den klassischen Erklärungsansätzen den berechtigten Vorwurf nicht hinreichender Erklärungsmodelle ein. „Das Einkommen mag im statistischen Sinne ein gutes Explanans sein; der statistisch gesicherte Zusammenhang von Einkommen mit Mortalität wie Morbidität bleibt trotzdem erklärungsbedürftig, also ,Explanandum'“(Behrens 2006: 54).

Zur Lösung dieses Erklärungsproblems könnte das Konzept des Sozialkapitals beitragen. Die Gesundheitsforschung ist zunehmend an der Frage interessiert, ob sich die sozialen Herkunftseffekte auf die Gesundheit und das Gesundheitshandeln über soziale Beziehungen erklären lassen (Chen et al. 2009: 306; D'Hombres et al. 2010: 56; Hawe und Shiell 2000: 871). Bisherige Studien bestätigen zwar die Gesundheitswirksamkeit von Sozialkapital auf individueller und kollektiver Ebene (Poortinga 2006: 256; Snelgrove et al. 2009: 1993), die Zahl an soziologischen Studien zur Thematik ist aber überschaubar (Kroll und Lamp 2007: 122). Zudem sind mit der disziplinübergreifenden Ausweitung des Konzepts theoretische wie methodische Schwierigkeiten verbunden. Ein Hauptkritikpunkt ist die fehlende Klarheit bezüglich dessen, was unter Sozialkapital überhaupt zu verstehen ist, wie es gemessen, operationalisiert und theoretisch umfassend eingebunden werden kann (Chen et al. 2009: 306; Derose und Varda 2009; Diekmann 2007: 48; Diewald 1991: 83; Franzen und Pointner 2007: 66; Fuku-

\footnotetext{
${ }^{50}$ Die Unterscheidung zwischen Selektions- und Verursachungshypothese geht auf eine Forschergruppe unter Vorsitz von Sir Douglas Black zurück, die 1977 von der britischen Regierung den Auftrag erhielt, soziale Ungleichheiten in der Mortalität und Morbidität zu untersuchen und Maßnahmen zu ihrer Verringerung zu erarbeiten (Richter und Hurrelmann 2006: 18).

${ }^{51}$ Selektionsprozesse können nicht primär für das soziale Gefälle in der Gesundheit verantwortlich sein, weil „die Anzahl der von vertikaler sozialer Mobilität betroffenen Personen, zu gering ist, um entscheidenden Einfluss auf die beobachteten Unterschiede in der Gesundheit auszuüben“ (Richter 2005: 139). Zudem verstärken sich die sozialen Differenzen im Krankheitsgeschehen mit zunehmendem Lebensalter nicht und die Mortalitätsunterschiede nehmen im Vergleich zum Ausmaß sozialer Auf- und Abstiege stärker zu. Zudem mildern sozialund gesundheitspolitische Instrumente soziale Abwärtsmobilität (Jungbauer-Gans 2002: 32).
} 
yama 1997: 377; Hawe und Shiell 2000: 877; Jungbauer-Gans 2002: 39; Koob 2007: 15, 25f.; Lüdicke und Diewald 2007: 12).

Bevor die Hypothesen zum Einfluss sozialen Kapitals auf die Gesundheit abgeleitet werden, soll deshalb darauf eingegangen werden, was darunter verstanden wird und welche Bedingungen an einen möglichen Erklärungserfolg des sozialen Gesundheitsgradienten durch den Sozialkapitalansatz geknüpft sind.

\subsection{Das Sozialkapitalkonzept in der Gesundheitsforschung}

\subsubsection{Zum Sozialkapitalbegriff}

Die erfolgreiche Etablierung des Sozialkapitalbegriffes in der Soziologie, Ökonomie und Politikwissenschaft ist vor allem ein Verdienst von Bourdieu (1983), Coleman (1988, 1990) und Putnam (1993, 1995). In den vergangenen Jahrzehnten differenzierten sich dabei statt einer kohärenten Sozialkapitaltheorie mehrere Forschungsparadigmen aus, die Sozialkapital entweder als individuelle oder als gesellschaftliche Ressource konzipieren (Diewald 2007: 183; Franzen und Pointner 2007; Haug 2007: 86; Klocke 2004: 90; Koob 2007: 18). Für die vorliegende Fragestellung eignet sich das Konzept des Sozialkapitals als individuelle Ressource, zu der Individuen durch die Einbindung in soziale Beziehungen Zugang erhalten. Ein solches Begriffsverständnis geht auf Bourdieu (1983) zurück, dessen Werk zunehmend wissenschaftliche Aufmerksamkeit in der Gesundheitsforschung zukommt (Derose und Varda 2009: 272).

Relevant für die vorliegende Forschungsfrage sind auch Überlegungen aus der gesundheitspsychologischen Stress- und Unterstützungsforschung, die auf eine drei Jahrzehnte umfassende wissenschaftliche Auseinandersetzung mit der Frage nach dem gesundheitlichen Wert sozial unterstützender Beziehungen zurückblicken kann (Jungbauer-Gans 2002: 42; Mielck und Bloomfield 2001). Die Gesundheitswirksamkeit sozialer Beziehungen wird in diesem Forschungsbereich über drei soziale Prozesse - soziale Unterstützung, Beziehungskonflikte, soziale Regulierung und Kontrolle - erklärt. Soziale Unterstützung wird definiert als ,support accessible to an individual through social ties to other individuals, groups, and the larger community“(Lin et al. 1979: 109), gemeint ist emotionale und instrumentelle Hilfe, die Individuen aus sozialen Beziehungen ziehen können; Konflikte beziehen sich auf die möglichen negativen Aspekte sozialer Eingebundenheit und soziale Regulation und soziale Kontrolle stehen für die regulierende Qualität sozialer Beziehungen (House et al. 1988: 239).

Der soziale Unterstützungsaspekt findet sich auch in der soziologischen Forschung zu Sozialkapital wieder. Portes (1998: 7 f.) identifiziert in einem Überblick über gängige Sozialkapitalkonzepte und -anwendungen in der Soziologie drei Funktionen, die Sozialkapital zugeschrieben werden: die Funktion der familiären und verwandtschaftlichen Unterstützung, die Funktion der sozialen Kontrolle und die Funktion des Ressourcenzugangs über außerfamiliäre Netzwerke. Hier gibt es offensichtlich Schnittmengen zwischen den Konzepten der soziologischen und psychologischen Gesundheitsforschung. Gemein ist ihnen nicht nur die Vorstellung von Sozialkapital als Ressource, die dem Individuum Handlungsfähigkeit und (gesundheitli- 
chen) Statuserwerb/-erhalt ermöglicht, deren Zugang aber von dem Unterhalt sozialer Beziehungen abhängt (Bourdieu 1983: 190f.; Coleman 1988: 99; Coleman 1991: 392), sondern zudem die geteilte Annahme darüber, dass soziale Unterstützung ausschließlich in den sozialen Beziehungen existiert. Für den Aufbau dieser Beziehungen müssen Ressourcen (Energie, Geld, Zeit) eingesetzt werden. Dies gilt vor allem für nicht-verwandtschaftliche und weniger für verwandtschaftliche Beziehungen, weil diese „,mit der Geburt vorgegeben [sind], d.h. der Mensch wächst in sie hinein, wodurch wenig Beziehungsarbeit investiert werden muss.“ (Rosenbaum 1998: 29). Gleichzeitig haben verwandtschaftliche Beziehungen anders als nichtverwandtschaftliche Beziehungen unmittelbar einen normativen Charakter (ebd.: 29). Deshalb ist auf die Unterstützung durch Verwandte und vor allem Eltern eher Verlass, als durch nichtverwandtschaftliche Beziehungen.

\subsubsection{Die gesellschaftliche Verteilung sozialen Kapitals}

Wenn Sozialkapital als Erklärungsansatz für schichtspezifisches Gesundheitsverhalten taugen soll, muss eine Ungleichverteilung zwischen den Sozialschichten vorhanden sein. Die Ergebnisse neuerer Untersuchungen stellen in Bezug auf Beziehungsstrukturen allerdings kein einheitliches sozial strukturiertes Verteilungsmuster fest (Jungbauer-Gans 2002: 42). Im Einklang mit den Überlegungen Bourdieus (1983) nimmt der Umfang an Sozialkapital eher mit zunehmender Schichtzugehörigkeit zu. Sozial Bessergestellte verfügen also über die größeren und vielfältigeren sozialen Netzwerke und insbesondere über mehr sog. ,,weak ties“. Nun ist aber anzunehmen, dass der nachweisliche Beziehungsvorteil höherer Sozialschichten bezüglich der „weak-ties“, sogenanntem überbrückendem Sozialkapital, nicht zwangsläufig einen Vorteil beim Aufziehen von Kindern bedeuten muss. Wegen der Spezifität sozialen Kapitals kann eine bestimmte Form von Sozialkapital förderlich in einem Handlungskontext sein, in einem anderen jedoch wertlos oder sogar schädlich (Coleman 1988: 98). Für die Bewältigung der Aufgaben, die ab der Geburt eines Kindes für die Eltern anfallen, ist anzunehmen, dass verwandtschaftliche, also starke soziale Beziehungen von größerer Bedeutung sind.

Hinsichtlich des Umfangs enger verwandtschaftlicher Beziehungen bestehen kaum soziale Unterschiede (Jungbauer-Gans 2002: 39, 42). Historische Studien deuten aber darauf hin, dass verwandtschaftliche Hilfsleistungen in höheren Schichten eher ökonomischer Natur sind, während in niedrigen Schichten die Eigenschaft von Verwandtschaft als soziales Netz dominiert, welches praktische Unterstützung lieferte (Häußermann und Siebel 1996). Wir nehmen daher an, dass es für Eltern aus den unteren Sozialschichten eher möglich sein sollte, soziale Ressourcen der Familienmitglieder in Form von familiärer Unterstützung (z.B. durch die zeitweilige Übernahme von Kinderbetreuung) für sich nutzbar zu machen. Darüber hinaus ist zu vermuten, dass höhere Sozialschichten ein mögliches strukturelles Defizit an verwandtschaftlichen Beziehungen im näheren Wohnumfeld zum Teil durch eine stärkere Inanspruchnahme von institutionellen Betreuungsangeboten kompensieren können, da sie sich diese im Durchschnitt finanziell eher leisten können. Für das hier betrachtete Lebensalter (Neugeborenenalter) dürfte außerfamiliäre Betreuung aber nur eine untergeordnete Rolle spielen. 
$\mathrm{Zu}$ Ungunsten sozioökonomisch benachteiligter Gruppen sollte sich allerdings auswirken, dass in unteren Schichten im Mittel mehr Mütter alleinerziehend sind (Kurz und Becker 2017: 180). Durch die häufigere Abwesenheit des Vaters im Haushalt lässt sich also ein Integrationsdefizit vermuten, wobei starke Beziehungen zu weiteren Familienmitgliedern, wie Großeltern, Geschwistern oder engen Freunden diesen Nachteil möglicherweise kompensieren.

\subsection{Hypothesenentwicklung}

Der primäre Fokus des Beitrages liegt auf der Relevanz von sozialen Beziehungen der Mutter für die Inanspruchnahme von Vorsorgeuntersuchungen für ihr Kind (bis zum Alter von 18 Monaten). Darüber hinaus sollen aber andere zentrale persönliche und materielle Faktoren, die das Engagement der Mutter beeinflussen könnten, nicht gänzlich vernachlässigt werden. ${ }^{52}$

$\mathrm{Zu}$ den Faktoren, die neben sozialen Beziehungen einbezogen werden, gehören (a) die subjektive Zufriedenheit mit der Mutterrolle und das Zurechtkommen mit den Anforderungen der Mutterrolle und (b) die zeitlichen Ressourcen, die einer Mutter zur Verfügung stehen. Indirekt soll auch über das Schulbildungsniveau der Mutter (c) deren gesundheitsbezogenes Wissen berücksichtigt werden. Diese genannten - im Sinne von Sozialkapital des Kindes zu interpretierenden - Ressourcen begünstigen neben materiellen Ressourcen das Ausüben gesundheitsförderlichen Verhaltens (Jungbauer-Gans 2002: 37 - 39). Materielle Faktoren, wie die finanzielle Ressourcenausstattung, dürften für das Präventivhandeln jedoch von nebengeordneter bzw. indirekter Bedeutung sein, da die gesetzlich verankerten Vorsorgeuntersuchungen bei altersgemäßer Teilnahme kostenlos angeboten werden.

Eine Schlüsselfunktion für den Vorsorgebesuch besitzen demgegenüber allgemeines Wissen zur Förderung der Gesundheit sowie spezifisches Wissen zu Vorsorgeuntersuchungen und ihre Relevanz für die frühzeitige Identifizierung entwicklungsbezogener Auffälligkeiten (kognitiv, wie motorisch und mental) und Krankheiten bei Kindern (Thaiss et al. 2010). Daneben spielen allgemeine Erziehungskompetenzen sowie Kompetenzen im Umgang Alltagstressoren, welche die Betreuung, Erziehung, Fürsorge und Pflege neugeborener Familienmitglieder mit sich bringt. Persönliche für das Vorsorgehandeln relevante Ressourcen beziehen sich demnach vor allem auf gesundheitskulturelles Kapital sowie auf persönliche Kompetenzen für die Bewältigung der Anforderungen an die Elternrolle. Aufgrund der Korrelatin mit Bildung sollte zudem die Klassenposition mit dem Vorsorgebesuch zusammenhängen.

Darüber hinaus kann mithilfe der Familienökonomie (Becker 1981) argumentiert werden, dass in Gesundheitsfragen, das heißt, in Fragen zu Erhalt, Förderung und Vorsorge, Zeit eine wichtige persönliche Handlungsressource oder Restriktion darstellt (Becker und Kurz 2011: 283). Nachfolgend soll das Augenmerk nun auf die Wirksamkeit sozial unterstützender Beziehungen im Familien- und Verwandtschaftskreis gelenkt werden. Hierfür gilt es kapitaltheo-

\footnotetext{
${ }^{52}$ Eine Einengung auf soziale Beziehungen und Ressourcen allein der Mutter (und nicht beider Elternteile), erfolgt aufgrund der Datenlage (vgl. Abschnitt 5). Dies stellt jedoch keine gravierende Einschränkung dar, da gerade in der frühen Lebensphase des Kindes in aller Regel die Mutter die zentrale Bezugsperson des Kindes ist.
} 
retisch differenziert aufzuzeigen, wie von Müttern besessenes Beziehungskapital die Bewältigung gesundheitsbezogener Erziehungsanforderungen (Vorsorgehandeln) beeinflusst.

Die zentrale Grundannahme lautet, dass die Integration in soziale Beziehungen für die Aufrechterhaltung und Förderung von Gesundheit der nachwachsenden Generation von Bedeutung ist. Verschiedene Studien zeigen, dass es einen robusten Zusammenhang zwischen Sozialkapital und gesundheitsförderlichem Verhalten und guter Gesundheit gibt (Drukker et al. 2005; Kawachi et al. 1999; Lindstrom 2005; Lundborg 2005; Mohensi und Lindstrom 2007).

\subsubsection{Sozialkapital der Mutter}

Als klassischer Bezugspunkt medizinsoziologischen Wissens machte bereits Durkheim (1970) auf den positiven Einfluss sozialer Integration auf die Gesundheit aufmerksam (House et al. 1988: 296; Siegrist 1995: 14). Praktisch bedeutet die soziale Integration der Mutter, dass diese durch die Einbindung in soziale Beziehungen soziale Unterstützung erfährt. Gleichzeitig bewirkt die Einbindung in soziale Beziehungen auch soziale Kontrolle, wodurch normengerechtes Handeln, z.B. in Form der Teilnahme an Vorsorgeuntersuchungen gefördert werden sollte. Soziale Integration erscheint besonders in Situationen außergewöhnlicher Belastungen bedeutsam. Die Geburt eines Kindes stellt ein kritisches Lebensereignis dar, das häufig zu einem Gefühl der Überforderung der Mutter führt, unabhängig davon, ob der Umgang mit dem Kind genossen wird. Die Belastungen resultieren auch daraus, dass die tatsächlichen Anforderungen des Mutter- und Vaterdaseins häufig zunächst unterschätzt werden (Bründel und Hurrelmann 1996: 25). Alleinerziehende, die sich in keiner Partnerschaft befinden, sind dabei aufgrund ihrer alleinigen oder hauptsächlichen Zuständigkeit für Kind und Haushalt (und eventuell für das Einkommen) in einer besonders schwierigen Situation. Denn diese Aufgaben erfordern Zeit und Kraft, die in Einelternfamilien besonders knappe Güter sind (Helfferich et al. 2003). Gesundheitsförderndes Handeln könnte daher für Alleinerziehende schwerer als für andere Mütter zu realisieren sein. Die erste Hypothese lautet damit:

H1: Wachsen Kinder mit nur einem Elternteil, dann nehmen sie im Vergleich zu Kindern, die mit beiden Elternteilen aufwachsen, seltener an Vorsorgeuntersuchungen teil.

Bei Bestehen einer Partnerschaft kann die Unterstützung durch den Partner unterschiedlich stark ausfallen. Dabei kann zwischen emotionaler und instrumenteller Unterstützung durch den Partner unterschieden werden, wobei wir keine begründeten Vermutungen darüber haben, welcher Unterstützungsaspekt relevanter ist. Es lässt sich eine weitere Hypothese formulieren:

H2: Je mehr sich Mütter von ihrem Partner (a) emotional und (b) instrumentell unterstützt fühlen, desto eher findet eine Teilnahme an Vorsorgeuntersuchungen statt.

Zwar ist zu vermuten, dass Alleinerziehende im Allgemeinen weniger soziale Unterstützung erfahren, da sie im Alltag keine oder nur geringfügige Unterstützung durch einen Partner haben, allerdings ist denkbar, dass diese fehlende Unterstützung durch andere soziale Beziehungen im Freundes- oder Verwandtschaftskreis kompensiert wird. Aufgrund der Datenlage wird sich aber nur auf Personen aus dem Verwandtschaftskreis und auch nur auf die instrumentelle 
Unterstützung konzentriert. Zumindest die Einschränkung auf Verwandte erscheint nicht sehr problematisch, da soziale Unterstützungsleistungen für Familien mit Kindern vor allem durch den engeren Verwandtenkreis geleistet werden. Einen positiven Gesundheitseffekt verwandtschaftlicher Unterstützung vermuten wir deshalb, weil umfassende Unterstützungsressourcen eine (zeit)entlastende Funktion haben (Bühler 2007: 407). Es ist demnach anzunehmen, dass Mütter durch die zeitweilige Übernahme der Kinderbetreuung durch Dritte persönliche für die physische und mentale Erholung bedeutsame Freiräume gewinnen und sich die so entstehenden regenerativen Potenziale positiv auf die Aktivitäten, die zum Kindeswohl unternommen werden, auswirken. Darüber hinaus begünstigen Familienmitglieder über soziale Kontrolle gesundheitsförderliches Handeln (House et al. 1988). Zu erwarten ist daher Folgendes:

H3: (a) Je größer die Anzahl Personen im Verwandtschaftskreis ist, die bei der Kinderbetreuung unterstützt, und (b) je mehr Stunden pro Woche Kinderbetreuung durch Verwandte übernommen wird, desto wahrscheinlicher ist die Teilnahme an Vorsorgeuntersuchungen.

Die Hypothesen sollten sowohl für Alleinerziehende als auch für Mütter in Partnerschaft gelten. Allerdings ist zu vermuten, dass der gesundheitsförderliche Aspekt für Alleinerziehende bedeutsamer ist, da sie nicht auf einen (unterstützenden) Partner zurückgreifen können.

\subsubsection{Sonstige Ressourcen der Mutter}

Auch die eigenen Ressourcen und Kompetenzen der Mutter sollten von zentraler Bedeutung für ihr Gesundheitshandeln in Bezug auf das Kind sein. Hierbei kann als erstes angenommen werden, dass eine Mutter um so eher gesundheitsförderlich für ihr Kind handeln wird, je besser sie mit der Mutterrolle zurechtkommt und je zufriedener sie mit der Mutterrolle ist. Anzunehmen ist auch, dass die Enge der Beziehung zwischen Mutter und Kind die gesundheitliche und psychische Entwicklung des Kindes positiv beeinflusst (Petermann et al. 2000: 27). So wird vermutetet, dass eine in der Schwangerschaft gegebene Unerwünschtheit des Kindes zu einer weniger starken Eltern-Kind-Beziehung führt, welche potentiell das familiäre Klima und die Entwicklung des Kindes stören kann (Jungbauer-Gans und Kriwy 2004: 17). Die genannten Einflussgrößen können unter dem Begriff ,psychosoziales Wohlbefinden in Bezug auf die Mutterrolle“ zusammengefasst werden. Die entsprechende Hypothese lautet:

H4: Je besser das psychosoziale Wohlbefinden der Frau in Bezug auf ihre Mutterrolle ist, desto eher nimmt sie die kindbezogenen Vorsorgeuntersuchungen in Anspruch.

Darüber wird erwartet, dass die zeitliche Verfügbarkeit der Mutter (und des Vaters) für das Kind eine entscheidende Bedingung für den Zugang zum Humankapital und den gesundheitsbezogenen Aktivitäten der Eltern ist (Coleman 1988). Zeit und Aufmerksamkeit für das einzelne Kind sollten mit steigender Geschwisterzahl abnehmen, was in der Folge Auswirkungen auf das Ausmaß der gesundheitsbezogenen Aktivitäten haben könnte. Ebenso ist zu erwarten, dass sich im Mutterschutz oder Elternzeit befindende Mütter über größere zeitliche Spielräume verfügen, als erwerbstätige Mütter. Folglich wird eine weitere Hypothese formuliert: 
H5: Je mehr Zeit der Mutter für das einzelne Kind zur Verfügung steht, desto wahrscheinlicher ist die Teilnahme an Vorsorgeuntersuchungen für Kinder.

Insbesondere für die Hypothesen 2, 3 und 4 ist zu bedenken, dass Endogenitätsprobleme auftreten können: Das psychosoziale Wohlbefinden der Mutter kann gemindert sein, weil die Gesundheit des Kindes schlecht ist. Und die Unterstützung durch Partner und Verwandte ist vielleicht hoch, weil das Kind erkrankt ist. Gleichzeitig hat die Gesundheit des Kindes vermutlich einen direkten Einfluss auf die Teilnahme an den U-Untersuchungen. Um dem Endoginitätsproblem zu begegnen, werden in den späteren Regressionsmodellen Indikatoren zur objektiven körperlichen Gesundheit des Kindes (bei der Geburt festgestellte Störungen) und Schwangerschaftskomplikationen (pränatale Muttergesundheit) berücksichtigt.

\subsection{Daten und Variablen}

Das Sozioökonomische Panel (SOEP) stellt für die Analyse von gesundheitlichen Ungleichheiten eine geeignete Datenbasis dar (SOEP Group 2001). Entsprechend der Zielsstellung, Mechanismen der frühen Entstehung gesundheitlicher Ungleichheit zu identifizieren, basieren die folgenden Analysen auf den Daten des Neugeborenenfragebogens 2003 bis 2008. Um die Teilnahme am Krankheitsfrüherkennungsprogramm für Kinder differenziert unter dem Ungleichheitsaspekt auswerten zu können, werden die in separaten Personeninterviews erhobenen Daten zur sozialen Situation der Mütter den Gesundheitsdaten der Kinder zugespielt. Insgesamt liegen Informationen zur Gesundheit von 1435 Neugeborenen und 1179 Müttern vor. Von diesen Müttern sind 256 mehrfach $^{53}$ (17,8\%) im Datensatz vertreten. Die Ausgangsfallzahl von 1435 Befragten wird deshalb auf die Primärfälle ( $N=1179 ; 82,2 \%)$ reduziert, so dass jede Mutter nur einmal mit einem Kind - jenem, über das die Mutter erstmalig im Rahmen der Mutter-Kind-Befragung berichtete - im Datensatz verbleibt. Es werden des Weiteren nur jene Befragten berücksichtigt, für die in den abhängigen und unabhängigen Variablen keine fehlenden Werte vorliegen. Davon abgewichen wurde bei den Variablen zur Bildung der Mutter und zur Klassenlage, weil diese vergleichsweise viele fehlende Werte aufweisen. Zur Vermeidung von Selektivitätsproblemen wurden die Fälle mit fehlenden Angaben in diesen Variablen in der Analyse belassen und über Dummy-Variablen statistisch kontrolliert. Für die empirische Analyse verblieben damit 1028 Kinder im Alter zwischen 0 und 18 Monaten (arithmetisches Mittel des Alters: 7,03 Monate, Standardabweichung: 3,76) in der Untersuchungsstichprobe (siehe Tabelle A 6.1 für einen Überblick zum Sample).

\subsubsection{Konstruktion der abhängigen Variablen}

Das elterliche Präventionsverhalten wird als abhängige Variable verwendet. Die Mütter sollten im Fragebogen angeben, welche die letzte durchgeführte ärztliche Vorsorgeuntersu-

\footnotetext{
${ }^{53}$ Von diesen Müttern sind $231(16,1 \%)$ mit zwei, 22 (1,5\%) mit drei und 3 Mütter mit vier $(0,2 \%)$ Kindern im Datensatz enthalten.
} 
chung $^{54}$ bei ihrem Kind war. Es kann somit geprüft werden, ob die Teilnahme an einer altersgemäßen Untersuchung erfolgte oder nicht $(\mathrm{Ja}=1$, Nein $=0)$.

\subsubsection{Konstruktion der unabhängigen Variablen}

Sozialkapital. Die Operationalisierung von Sozialkapital erfolgt über die familiären Beziehungen sowie die erbrachten Unterstützungsleistungen. Das Zusammenleben mit einem Partner wird über die Frage „Lebt der Vater des Kindes hier im Haushalt?“ ( Ja = 1, Nein = 0), die in der Mutter-Kind-Befragung gestellt wird, abgebildet. Bei der Messung der Unterstützung bei der Kindbetreuung erlauben die Daten eine Unterscheidung zwischen der Qualität - „Wie stark fühlen Sie sich von ihrem Partner bei der Betreuung des Kindes unterstützt?“ - und der Quantität instrumenteller Unterstützung: „Gibt es außer Ihnen andere Personen, die die Betreuung des Kindes zeitweise übernehmen? Wenn ja, welche Personen sind das und wie viel Stunden pro Woche übernehmen sie die Betreuung?“. Es konnte angegeben werden, ob der (Ehe-) Partner, die Großeltern oder ältere Geschwister des Kindes, andere Verwandte, eine Kinderkrippe, eine Tagesmutter oder andere Personen zeitweise die Kindbetreuung übernehmen. Die Abbildung des qualitativen Sozialkapitalaspektes erfolgt über die Dichotomisierung der ursprünglich fünf Unterstützungskategorien (sehr stark/ stark = 1, eher wenig/ gar nicht/ trifft nicht zu, gibt keinen Partner $=0$ ). Bei der Quantität der Betreuung (Stunden pro Woche) wird jeweils ein dichotomer Indikator bezogen auf den (Ehe-)Partner (0-5 Std. = 0, >5 Std. = 1) sowie auf Verwandte gebildet (Summe der Betreuungsstunden von Großeltern, Geschwistern und anderen Verwandten: 0-5 Std. =0, >5 Std. =1). Zusätzlich wird die Zahl der unterstützenden Verwandten mit einem Indikator erfasst. Außerfamiliäres Sozialkapital wird schließlich über eine Dummy-Variable zur Inanspruchnahme von Tagesmutter oder Kinderkrippe kontrolliert $(\mathrm{Ja}=1$, Nein $=0)$.

Das psychosoziale Wohlbefinden der Mutter wird über zwei Variablen zum körperlichen und seelischen Wohlbefinden der Frau im letzten Schwangerschaftsdrittel (Kategorien jeweils: sehr gut $=4$, gut $=3$, eher schlecht $=2$, sehr schlecht $=1$ ) additiv zusammengefasst (Cronbachs Alpha = 0,70) und durch die Anzahl der Items (zwei) dividiert. Diese neu gebildete Skala zum pränatalen Wohlbefinden (Mittelwerte: $1 ; 1,5 ; 2 ; 2,5 ; 3 ; 3,5 ; 4$ ) wird für die empirischen Analysen dichotomisiert (Mittelwerte 1 bis $3=0,3,5$ und $4=1$ ), wobei der Wert 1 eine sehr gute Muttergesundheit anzeigt. Zur Abbildung des psychosozialen Befindens der Frau nach der Geburt des Kindes gehen zwei Einstellungsskalen als binäre Variablen in die Analyse ein, zum einen das Item ,Ich fühle mich den neuen Aufgaben und Anforderungen oft nicht gewachsen“ $(0=$ stimme überhaupt nicht $\mathrm{zu}, 1=$ stimme eher nicht zu, stimme eher zu, stimme voll zu) und zum anderen das Item „Meine Rolle als Mutter ist für mich sehr befriedigend“ ( 1 = stimme voll zu, 0 = stimme eher zu, stimme eher nicht oder überhaupt nicht zu).

\footnotetext{
${ }^{54}$ Von den insgesamt 10 Vorsorgeuntersuchungen für Kinder werden sieben im frühen Kindesalter durchgeführt: U1 (bei Geburt), U2 (3.-10. Tag), U3 (4. - 5. Woche), U4 (3. - 4. Monat), U5 (6. - 7. Monat), U6 (10. - 12. Monat), U7 (21. - 24. Monat).
} 
Schließlich wird die Planung der Schwangerschaft $(1=\mathrm{Ja}, 0=$ Nein $)$ als (ein recht grobes) Proxy für die Erwünschtheit des Kindes operationalisiert. Die zeitliche Verfügbarkeit der Mutter für das Kind wird über die Variablen Mutterschutz oder Erziehungsurlaub bzw. Elternzeit ( $1=\mathrm{Ja}, 0=$ Nein) und die Anzahl der älteren Geschwister (0, 1, 2 und mehr) erfasst.

Sozialstruktur. Die soziale Herkunft der Neugeborenen wird über die Klassenlage des Elternhauses und die elterliche Bildungsnähe bestimmt. Für die Bestimmung der Klassenposition ist die berufliche Stellung des Vaters maßgeblich, wenn dieser zum Zeitpunkt der Befragung mit Mutter und Kind in einem Haushalt lebt. Bei fehlenden Vaterwerten oder bei alleinerziehenden Müttern wird auf die Klassenposition der Mutter zurückgegriffen. Aufgrund der geringen Fallzahl der Untersuchungsstichprobe wird das EGP-Klassenschema (Erikson und Goldthorpe 1992) auf fünf Kategorien reduziert. Es wird zwischen der Dienstklasse (1), nichtmanuellen Routinetätigkeiten (2), Selbständigen (3), FacharbeiterInnen, MeisterInnen und TechnikerInnen (4) sowie un- und angelernten ArbeiterInnen und LandarbeiterInnen (5) unterschieden. Die EGP-Klassen werden als Dummy-Variablen in der Auswertung berücksichtigt. Ergänzend wird im Falle von Nichterwerbstätigkeit eine entsprechende Variable aufgenommen. Des Weiteren wird der mütterliche Schulabschluss als unabhängige soziale Einflussgröße berücksichtigt. Hier unterscheiden wir zwischen dem Hauptschulabschluss (HS), der Mittleren Reife (MR) und der (Fach-)Hochschulreife (HR).

Kontrollvariablen. Neben den sozialen Herkunftsindikatoren wird die ethnische Herkunft der Mutter in die empirische Auswertung genommen. Dazu wird vom SOEP bereitgestellte Migrationsvariable „migback“ dichotomisiert (indirekter oder direkter Migrationshintergrund $=1$, kein (in)direkter Migrationshintergrund =0). Die regionale Herkunft zum Zeitpunkt der Befragung wird über die ebenfalls vom SOEP generierte Variable „\$sampreg“ operationalisiert (West $=1$, Ostdeutschland = 2). Zudem wird für das Lebensalter der Mutter (metrisch) und - auf Seiten des Kindes - das Geschlecht (Junge = 1, Mädchen =0), Alter (metrisch), Geburtsjahr (2002-2008, metrisch) und der objektive Gesundheitszustand (Krank = 1, Gesund $=0$ ) kontrolliert. Der Indikator zur Kindgesundheit basiert auf zwei Items: „Gab es bei Ihrem Kind in den ersten drei Monaten nach der Geburt ernste gesundheitliche Probleme, die einen Krankenhausaufenthalt notwendig machten?“ und „Wurden bei Ihrem Kind Verzögerungen, Störungen oder Behinderungen festgestellt?“. Bejahten die Mütter eine der beiden Fragen, nimmt die Gesundheitsvariable den Wert 1 an, ansonsten den Wert 0.

\subsection{Empirische Befunde}

Wie im Theorieteil bereits diskutiert wurde, ist eine zwischen Gesundheit und sozialer Lage vermittelnde Rolle von Sozialkapitalfaktoren an die Bedingung einer gesellschaftlichen Ungleichverteilung sozialen Beziehungskapitals geknüpft. Bevor die Befunde zur Gesundheitswirksamkeit sozialer Ressourcen auf das Vorsorgeverhalten berichtet werden, wird daher zunächst der Frage nachgegangen, ob sich - wie nach Bourdieu (1983) generell angenommen wurde - soziale Disparitäten hinsichtlich des Umfangs dieser Kapitalsorte feststellen lassen. 


\subsubsection{Sozialkapital: Ungleich verteilt nach Klassenlage und Bildung?}

Die in Abbildung 6.1 dargestellten deskriptiven Ergebnisse zur sozialen Verteilung der Sozialkapitalien bestätigen mit Ausnahme der verwandtschaftlichen Unterstützungsressourcen Bourdieus (1983) Überlegungen zu einer gesellschaftlichen Ungleichverteilung von Sozialkapital. Je höher die elterliche Klassenposition, desto eher lebt die Mutter mit dem Vater des Kindes zusammen. Die Qualität und Quantität der Unterstützung durch den Partner variieren aber nur tendenziell nach der Klassenzugehörigkeit. So fühlen sich Mütter aus der Dienstklasse $(81 \%)$ im Vergleich zu Müttern aus un- und angelernten Arbeiterhaushalten (74\%) in geringem Maße häufiger durch den Partner in der Kinderbetreuung unterstützt $\left(\mathrm{Chi}^{2}=2,409\right.$, $\mathrm{p}=0,121)$. Im Hinblick auf den zeitlichen Betreuungsumfang ist der prozentuale Abstand zwischen der Dienst- und unterer Arbeiterklasse (69\% vs. 54\%) von größerer statistischer Bedeutsamkeit $\left(\mathrm{Chi}^{2}=10.034, \mathrm{p}=0,002\right)$. Je höher die Klassenposition, desto eher übernimmt der Partner die Kinderbetreuung länger als fünf Stunden pro Woche. Wie theoretisch vermutet wurde (vgl. Jungbauer-Gans 2002), zeigen sich (nur) geringe soziale Unterschiede hinsichtlich des Umfangs verwandtschaftlicher Hilfsleistungen. ${ }^{55}$

Abbildung 6.1: Sozialkapital der Mutter nach elterlicher Klassenlage (in Prozent)

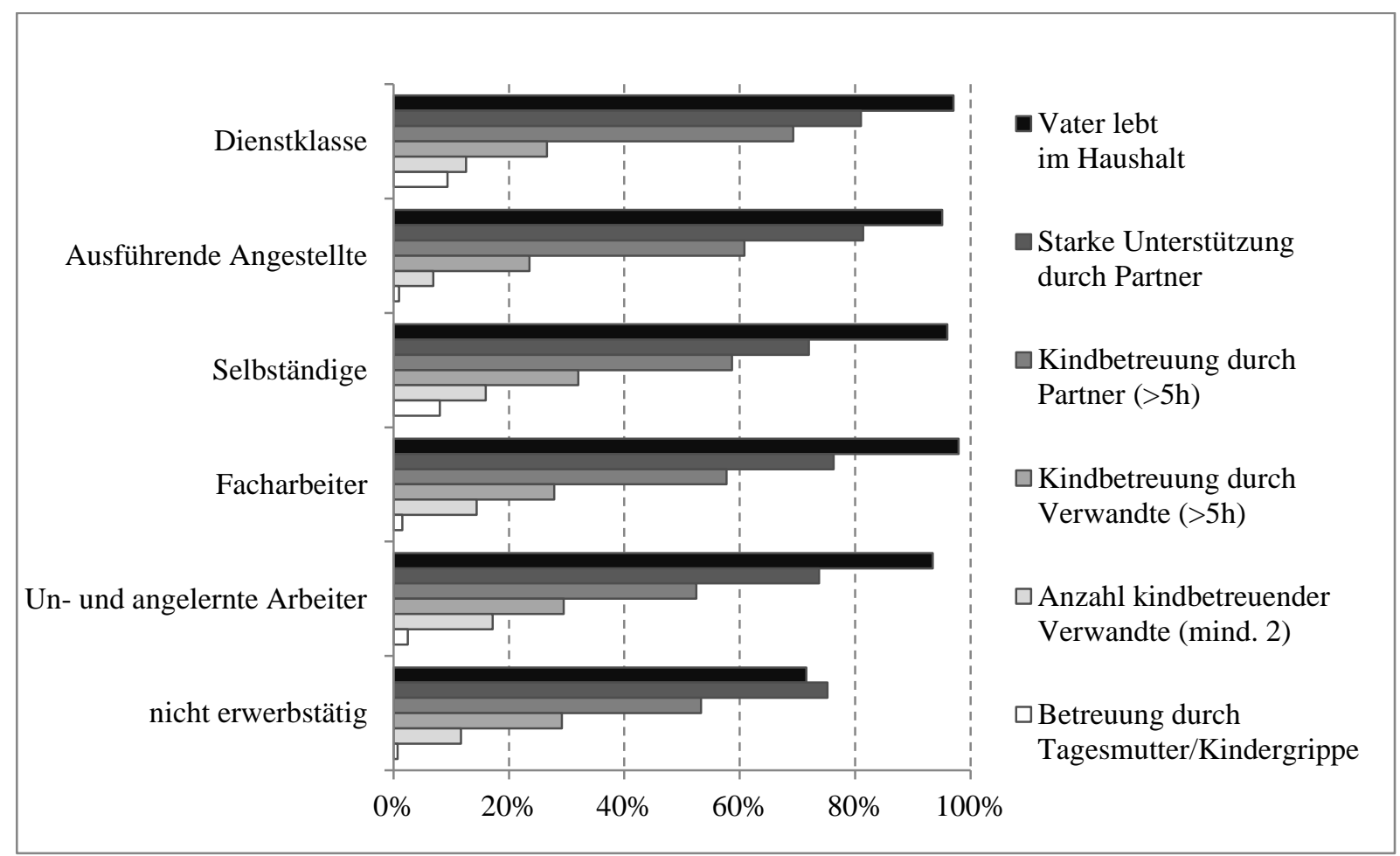

\footnotetext{
${ }^{55}$ Allerdings besteht ein negativer Zusammenhang zwischen dem verwandtschaftlichen Betreuungsumfang in Stunden und der mütterlichen Bildungsqualifikation: Mütter mit Hauptschulabschluss (33\%) und Mittlerer Reife (30\%) werden im Vergleich zu Müttern mit einer Hochschulzugangsberechtigung (24\%) häufiger mehr als fünf Stunden pro Woche bei der Kindsbetreuung durch Verwandte unterstützt $\left(\mathrm{Chi}^{2}=5,812\right.$, $\left.\mathrm{p}=0.055\right)$. Für die Anzahl der betreuenden Verwandten lassen sich jedoch keine Bildungsunterschiede feststellen.
} 
Abbildung 6.2 veranschaulicht soziale Disparitäten bezüglich der mütterlichen Ressourcen für das Kind. Je höher ihre Klassenposition, desto besser bewerten Mütter ihr körperliches und seelisches Befinden im letzten Drittel der Schwangerschaft $\left(\mathrm{Chi}^{2}=15.630, \mathrm{p}<0,01\right)$. Überraschenderweise sieht sich aber nur die Hälfte der Dienstklassenmütter den neuen Aufgaben und Anforderungen des Mutterdaseins gewachsen $\left(\mathrm{Chi}^{2}=4.917, \mathrm{p}<0,05\right)$ und empfindet die Mutterrolle, im Vergleich zu. Müttern aus Arbeiterhaushalten (Un- und Angelernte), seltener als uneingeschränkt befriedigend $\left(\mathrm{Chi}^{2}=6.300, \mathrm{p}<0,05\right)$. Gleichwohl ist die Prävalenz ungeplanter Schwangerschaften in unteren Klassen höher $\left(\mathrm{Chi}^{2}=70.645, \mathrm{p}<0,001\right)$, was die Wahrscheinlichkeit instabiler Familiensituationen erhöhen und möglicherweise auch die Enge der Mutter-Kind-Beziehung beeinflussen könnte. Klassenunterschiede hinsichtlich der Zeit, die Mütter mit ihrem Nachwuchs verbringen (gemessen über Mutterschutz, Erziehungsurlaub und Elternzeit), lassen sich mit den vorliegenden Daten nicht nachweisen. Allerdings gibt es eindeutige empirische Hinweise darauf, dass in Arbeiterfamilien eine größere Anzahl von Geschwisterkindern um die elterliche Aufmerksamkeit konkurriert $\left(\mathrm{Chi}^{2}=31,559, \mathrm{p}<0,001\right)$ : Während die Mehrheit (61\%) der in die Dienstklasse hineingeborenen Kinder kein weiteres Geschwisterkind hat, trifft dies nur auf 46\% der Un- und Angelernten und 53\% der Facharbeiterhaushalte zu.

Abbildung 6.2: $\quad$ Sonstige Ressourcen der Mutter nach elterlicher Klassenlage (in Prozent)

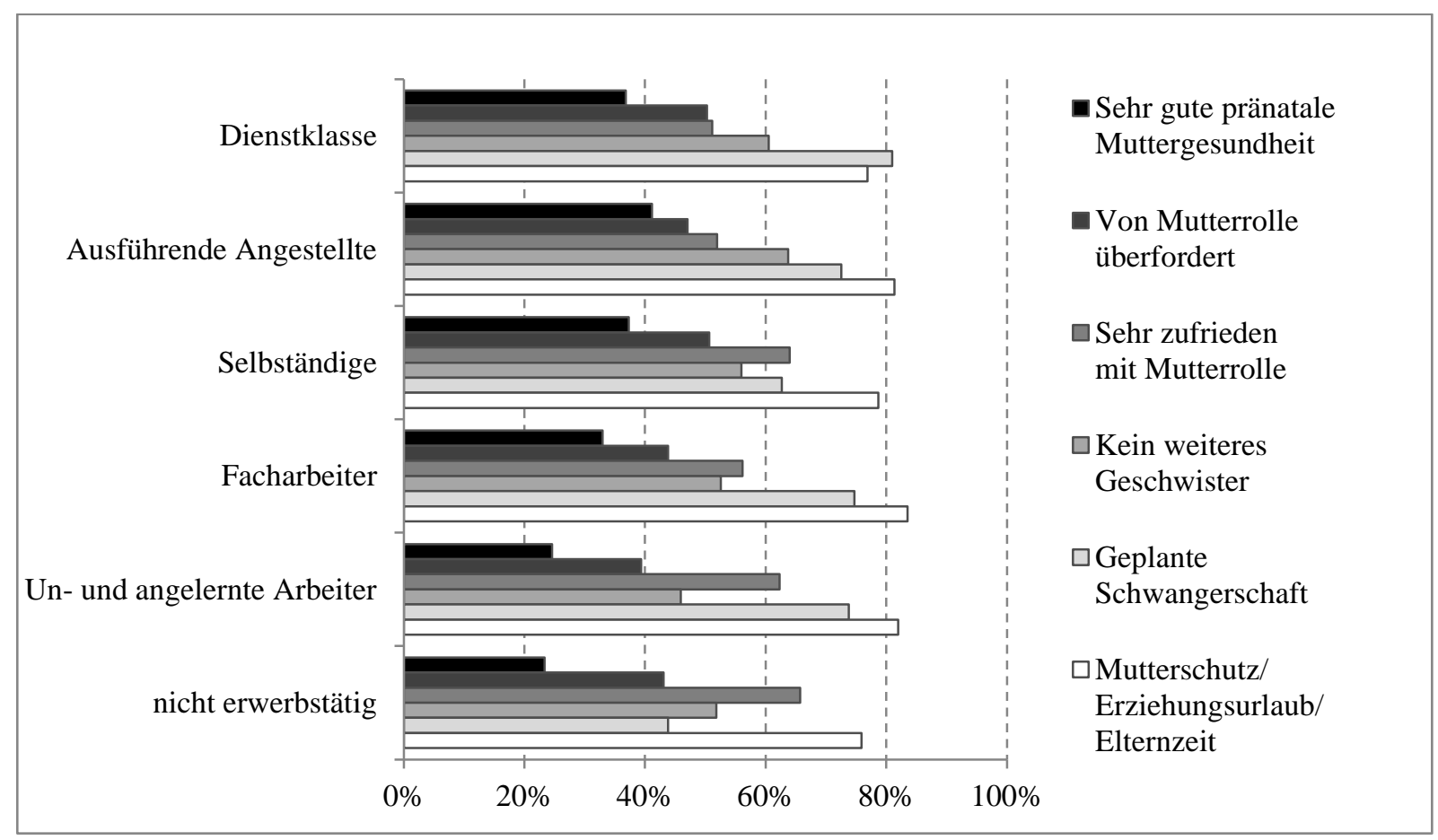

Zusammenfassend kann festgehalten werden, dass insgesamt eine gesellschaftliche Ungleichverteilung von familiärem Sozialkapital vorliegt. Ein sozial wenig privilegierter Hintergrund geht mit partnerschaftlichen Integrations- und instrumentellen Unterstützungsdefiziten einher. Im Hinblick auf die Verfügbarkeit verwandtschaftlicher Unterstützung lassen sich keine Klassendifferenzen nachweisen. Außerfamiliäre Betreuungsformen spielen in diesem frühen Lebensalter eine untergeordnete Rolle. Nur knapp 5\% der Neugeborenen werden außerfamiliär 
betreut und diese Betreuungsform wird fast ausschließlich von Eltern der Dienstklasse bzw. von bildungsnahen Elternhäusern in Anspruch genommen. Zu Ungunsten der unteren EGPKlassen ist das pränatale Wohlbefinden der Mütter ungleich verteilt, wobei die psychosoziale Bewältigung der Mutterrolle unteren Klassen jedoch im Mittel eher gelingt.

\subsubsection{Die Teilnahme an einer altersgemäßen Vorsorgeuntersuchung für Kinder}

Wie in Abbildung 6.3 ersichtlich, zeigt sich eine starke bildungsbezogene Abhängigkeit des Gesundheitshandelns $\left(\mathrm{Chi}^{2}=22,699, \mathrm{p}<0,001\right)$ : Mit steigender Schulbildung der Mutter wird die Beteiligung am Vorsorgeprogramm wahrscheinlicher (HS: 74,5\%, MR: 84,2\%, HR: 89,4\%). Demgegenüber hängt die Klassenlage des Elternhauses, wie Abbildung 6.4 zeigt, nicht signifikant mit dem Gesundheitshandeln zusammen $\left(\mathrm{Chi}^{2}=5,779, \mathrm{p}>0,10\right)$. Zwar besteht keine klare Rangfolge in den Teilnahmemustern zwischen den mittleren und höheren EGPKlassenkategorien. Nichtsdestotrotz wird deutlich, dass Kinder von un- und angelernten sowie von Eltern, die nicht erwerbstätig sind, am seltensten zur Gesundheitsvorsorge gehen. Neben der sozialen Herkunft entscheidet auch maßgeblich die ethnische Herkunft über die Teilnahme am Krankheitsfrüherkennungsprogramm für Kinder $\left(\mathrm{Chi}^{2}=24,268, \mathrm{p}<0,001\right)$. Mütter mit direkter oder indirekter Migrationserfahrung haben eine um 13 Prozentpunkte niedrigere Teilnahmequote als Mütter ohne Migrationshintergrund (73,8\% vs. 86,9\%). Dieser Befund steht im Einklang mit neueren Studien, die die ethnische Herkunft als eine wichtige Teilnahmedeterminante identifizieren (Stich et al. 2009).

Abbildung 6.3: Beteiligung am Vorsorgeprogramm nach mütterlichem Bildungsabschluss (in Prozent)

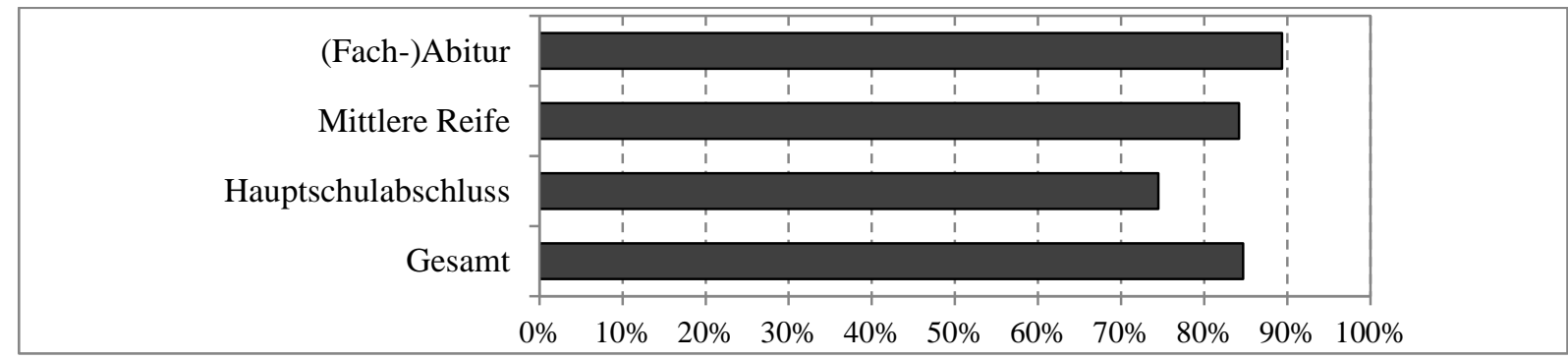

Abbildung 6.4: Beteiligung am Vorsorgeprogramm nach elterlicher Klassenlage (in Prozent)

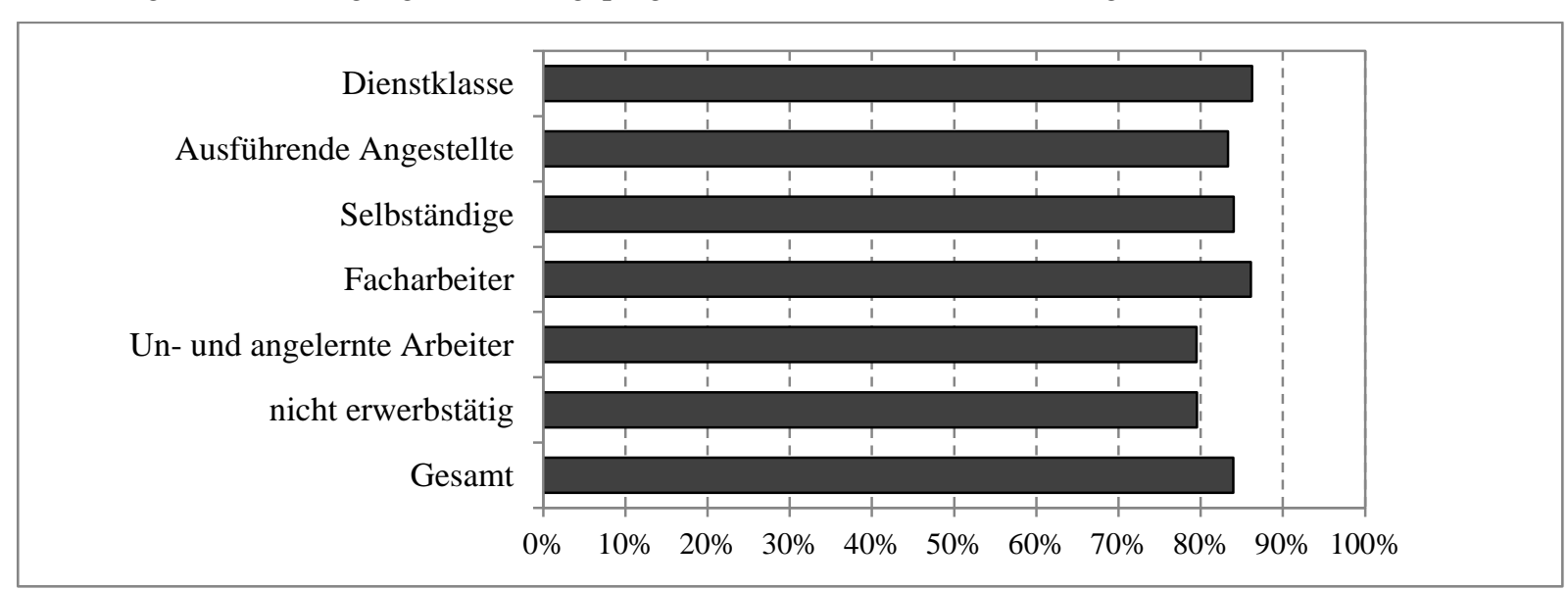


Welche Bedeutung hat nun soziales Kapital für die Inanspruchnahme präventiver Gesundheitsdienstleistungen für Kinder? Die in Abbildung 6.5 und 6.6 dokumentierten Ergebnisse stützen die Hypothesen zur Gesundheitswirksamkeit sozialen Kapitals aber nur zum Teil. Die Teilnahme an den U-Untersuchungen ist wahrscheinlicher, wenn Mütter mit dem Vater des Kindes zusammen leben (Hypothese 1 ; $\mathrm{Chi}^{2}=5.421, \mathrm{p}<0,05$ ) und ihr psychosoziales Befinden als gut bewerten (Hypothese 4), sich also den neuen Aufgaben und Anforderungen mit der Mutterrolle gewachsen fühlen $\left(\mathrm{Chi}^{2}=3.820, \mathrm{p}<0,10\right)$. War die Schwangerschaft geplant, nehmen Mütter eher an Vorsorgeuntersuchungen für ihre Kinder teil $\left(\mathrm{Chi}^{2}=3.061, \mathrm{p}<0,10\right)$. Für den Besuch der U-Untersuchungen statistisch unbedeutsam ist hingegen die Zufriedenheit mit der Mutterrolle ( $\left.\mathrm{Chi}^{2}=0.513, \mathrm{p}=0,474\right)$. Bezüglich der Relevanz zeitlicher Ressourcen für eine Teilnahme findet Hypothese 5 empirisch Unterstützung: Befindet sich die Frau in Mutterschutz, Erziehungsurlaub oder Elternzeit, fällt die Teilnahmewahrscheinlichkeit an den Vorsorgeuntersuchungen größer aus $\left(\mathrm{Chi}^{2}=18.481, \mathrm{p}<0,001\right)$, leben mehrere Geschwisterkinder im Haushalt hingegen niedriger $\left(\mathrm{Chi}^{2}=5.1603\right.$, $\left.\mathrm{p}<0,10\right)$. Keine Bestätigung finden die Annahmen zum positiven Einfluss familiärer Unterstützung auf das Gesundheitshandeln (Hypothesen 2 und 3). Die Vorsorgeteilnahme hängt widererwarten nicht davon ab, wie stark sich die Mutter durch den Partner unterstützt fühlt $\left(\mathrm{Chi}^{2}=0,362, \mathrm{p}>0,10\right)$. Als ebenso unerheblich erweisen sich die Stunden, in denen der Partner die Kinderbetreuung übernimmt $\left(\mathrm{Chi}^{2}=0,580\right.$, $\mathrm{p}>0,10)$. Unterstützung im Verwandtenkreis scheint sogar hinderlich für das Präventivhandeln: Je größer die Zahl betreuender Personen im Verwandtschaftskreis $\left(\mathrm{Chi}^{2}=2,815, \mathrm{p}<0,10\right)$ und der mit der Kinderbetreuung geleisteten Stunden pro Woche $\left(\mathrm{Chi}^{2}=9,528, \mathrm{p}<0,01\right)$, desto unwahrscheinlicher der Vorsorgebesuch. ${ }^{56}$

Abbildung 6.5: Beteiligung am Vorsorgeprogramm nach sozialem Kapital der Mutter (in Prozent)

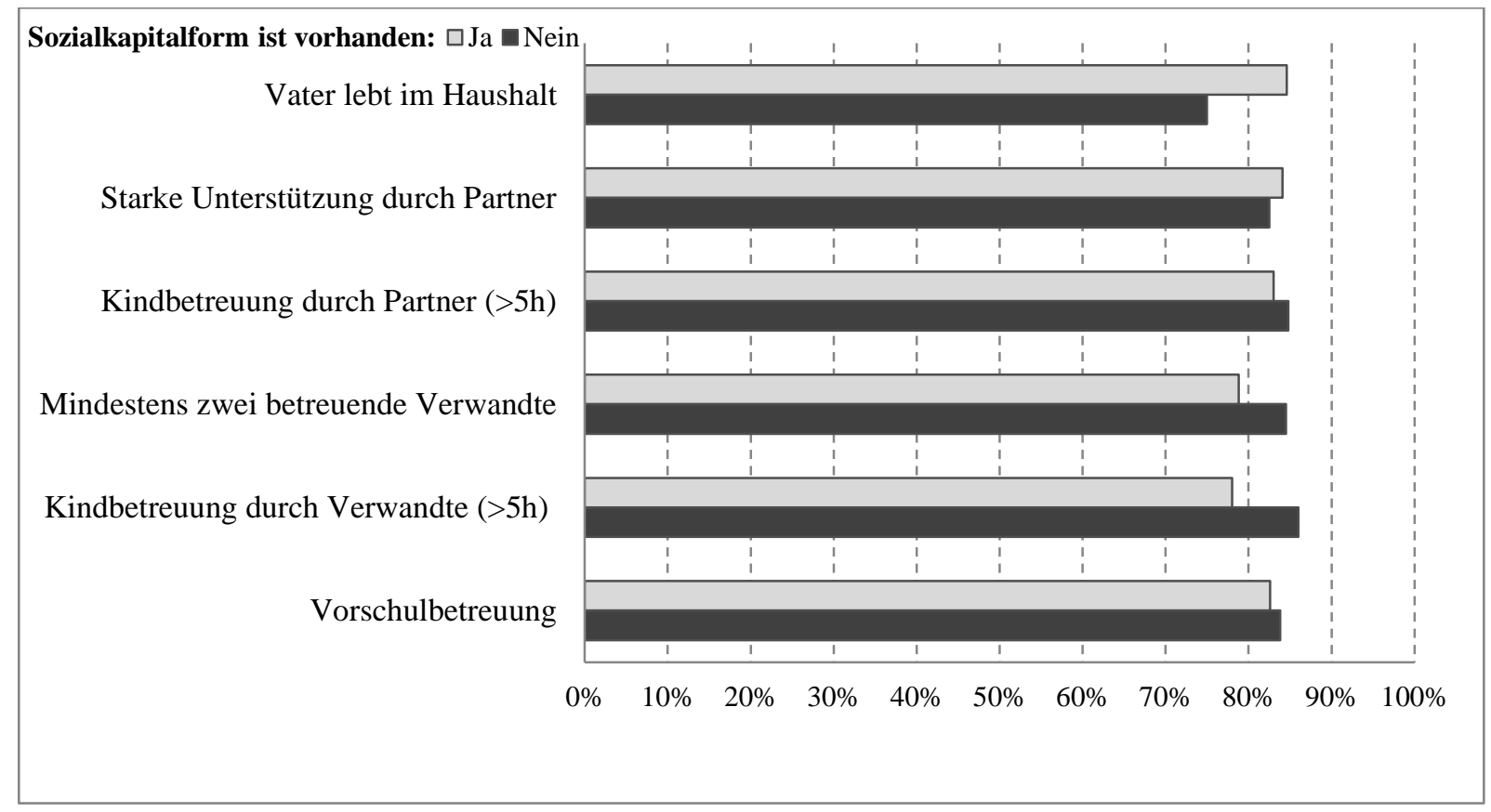

\footnotetext{
${ }^{56}$ Beide Unterstuitzungsmaße korrelieren stark miteinander (Pearson-R=0,552).
} 
Abbildung 6.6: Beteiligung am Vorsorgeprogramm nach sonstigen Ressourcen der Mutter (in Prozent)

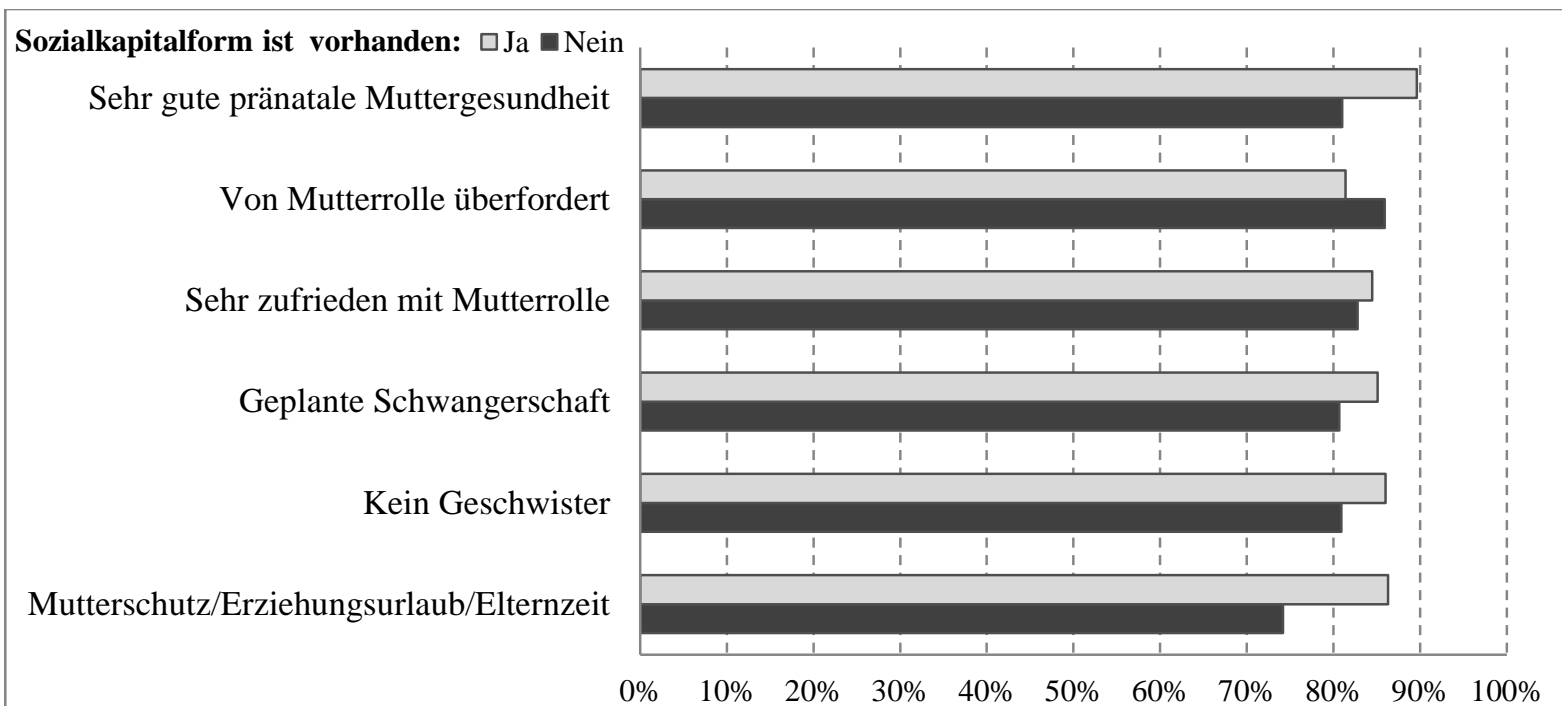

Im Folgenden wird nun geprüft, ob der Einfluss sozialer Unterstützung für Alleinerziehende und Mütter, welche mit dem Vater des Kindes zusammenleben, unterschiedlich stark ist. Hierzu schätzen wir logistische Regressionsmodelle (siehe Tabelle 6.1). Als Koeffizienten werden odds ratios - exp(bi) - und average marginal effects berichtet. Letztere ermöglichen einen Vergleich von hierarchischen Modellen (Winship und Mare 1984; Mood 2009).

Tabelle 6.1: Sozialkapitaleinfluss auf die Teilnahme an altersgemäßer Vorsorgeuntersuchung (logistische Regression, odds ratios und average marginal effects)

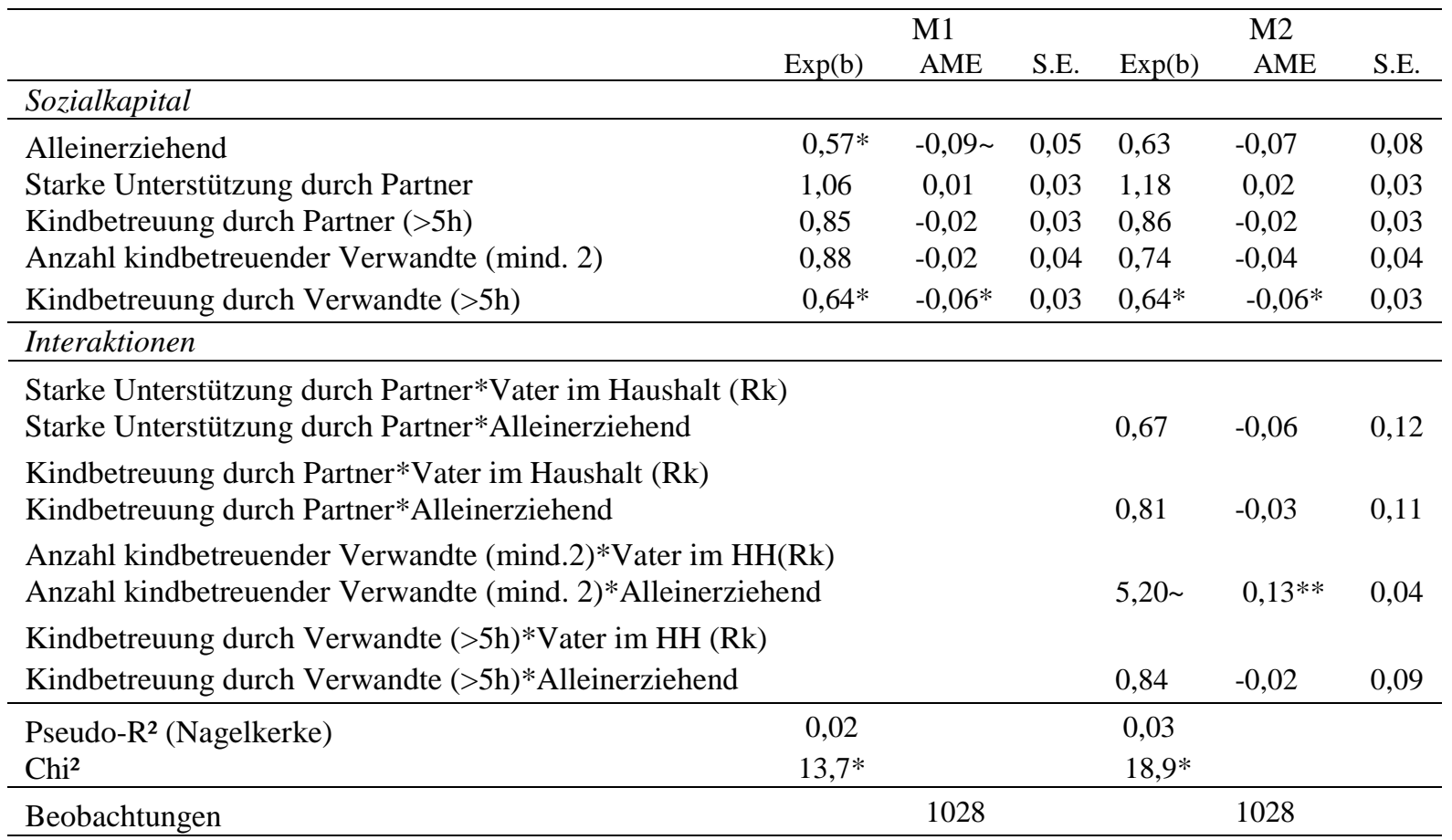

Quelle: SOEP 2003-2008, eigene Berechnungen.

Anmerkungen: AME = Average marginal effect, S.E. = Standardfehler (zu AME); Rk=Referenzkategorie; Modelle 1 und 2 kontrollieren für das Geschlecht des Kindes, das Alter der Mutter zum Zeitpunkt der Geburt, die Planung der Schwangerschaft sowie für die Region (Ost-/Westdeutschland). Keine dieser Variablen beeinflusst das Vorsorgeverhalten;

Signifikanzniveaus: $* * * p<0,001 ; * * p<0.01 ; * p<0.05 ; \sim p<0.10$; 
Modell 1 in Tabelle 6.1 zeigt eine um knapp die Hälfte $(0,57)$ reduzierte Teilnahmechance an Vorsorgeuntersuchen bei Kindern aus Einelternfamilien gegenüber jenen aus Zweielternfamilien. Signifikant niedrigere Teilnahmechancen haben außerdem Kinder, die mehr als fünf Stunden pro Woche von Verwandten betreut werden. Alle übrigen Sozialkapitalvariablen üben keinerlei signifikanten Einfluss aus. Mit Berücksichtigung der Interaktionseffekte zwischen Familienform und familiären Unterstützungsressourcen in Modell 2 wird deutlich, dass sich Unterstützung durch den Vater des Kindes für Mütter in beiden Familienformen ähnlich auswirkt. Gleichzeitig zeigt sich allerdings ein starker, wenn auch nur schwach signifikanter Teilnahmevorteil für Kinder Alleinerziehender gegenüber Kindern aus Zweielternfamilien, wenn mindestens zwei Personen aus dem Verwandtschaftskreis regelmäßig die Betreuung des Kindes übernehmen (odds ratio=5,20). Das heißt, die Teilnahmechancen an Vorsorgeuntersuchungen liegen bei Alleinerziehenden sogar höher als in Zweielternfamilien, wenn Alleinerziehende Unterstützung in der Kinderbetreuung aus dem Verwandtschaftskreis erhalten.

Mit den Modellen in Tabelle 6.2 wird nachfolgend der Frage nachgegangen, inwieweit Sozialkapital und sonstige Ressourcen der Mutter die Teilnahme an den U-Untersuchungen beeinflussen und inwieweit die Schichtabhängigkeit des Teilnahmeverhaltens durch diese GröBen bedingt ist. In Modell 1 werden lediglich die klassischen Sozialstrukturindikatoren Bildung und Klassenlage berücksichtigt. Die Ergebnisse dieses Modells belegen, dass die Teilnahmechancen der Neugeborenen an den U-Untersuchungen umso höher liegen, je bildungsnäher das Elternhaus ist, wobei Kinder mit ,Hauptschulmüttern“ besonders benachteiligt sind (odds ratio=0,34, $\mathrm{p}<0,001$ ). Die fehlenden Signifikanzen der Klassenlage-Variablen weisen auf eine vergleichsweise geringe Bedeutung der elterlichen Klassenlage für das kindbezogene Gesundheitshandeln hin. ${ }^{57}$ Entsprechend werden die Klassenlagenvariablen in den folgenden Modellen nicht mehr berücksichtigt. Modell 2 belegt, dass der Migrationshintergrund eine zentrale Determinante der Teilnahme an den U-Untersuchungen ist. Die Teilnahmechancen von Kindern mit Migrationshintergrund liegen etwa halb so hoch wie bei einheimischen Kindern (odds ratio $=0,45, \mathrm{p}<0,001$ ). Des Weiteren zeigt sich, dass die Teilnahmebereitschaft mit zunehmendem Kindesalter signifikant sinkt (odds ratio=0,93, p<0,01). Während dieser Effekt empirisch gut dokumentiert ist, überrascht der Anstieg der Teilnahmequote über die Zeit (odds ratio=1,11, $\mathrm{p}<0,10$ ). (Werden Mütter über die Zeit durch eine sich ausbreitende Ratgeberliteratur besorgter?) Allerdings ist dieser Effekt nicht in allen Modellen stabil. Für die Fragestellung ist nun von zentraler Bedeutung, ob sich die sozialen Ungleichheiten im Präventionsverhalten bezogen auf Bildung über die Sozialkapitalvariablen aufklären lassen. Dafür werden in Modell 3 zusätzliche Einflüsse der Sozialkapitalvariablen geschätzt.

\footnotetext{
${ }^{57}$ Schätzt man den Einfluss der elterlichen Klassenlage getrennt von der Schulbildung, zeigen sich auf dem 10\%-Signifikanzniveau negative Effekte für die Kategorien „un- und angelernte Arbeiter“ (odds ratio=0,62) und „,nicht erwerbstätig“ (odds ratio=0,62). Die Neutralisierung der EGP-Effekte nach Kontrolle der Bildungsnähe spricht dafür, dass die Teilnahmenachteile der unteren Klassen im Vergleich zur Dienstklasse teilweise auf unzureichendes gesundheitsrelevantes Wissen zurückzuführen sind.
} 


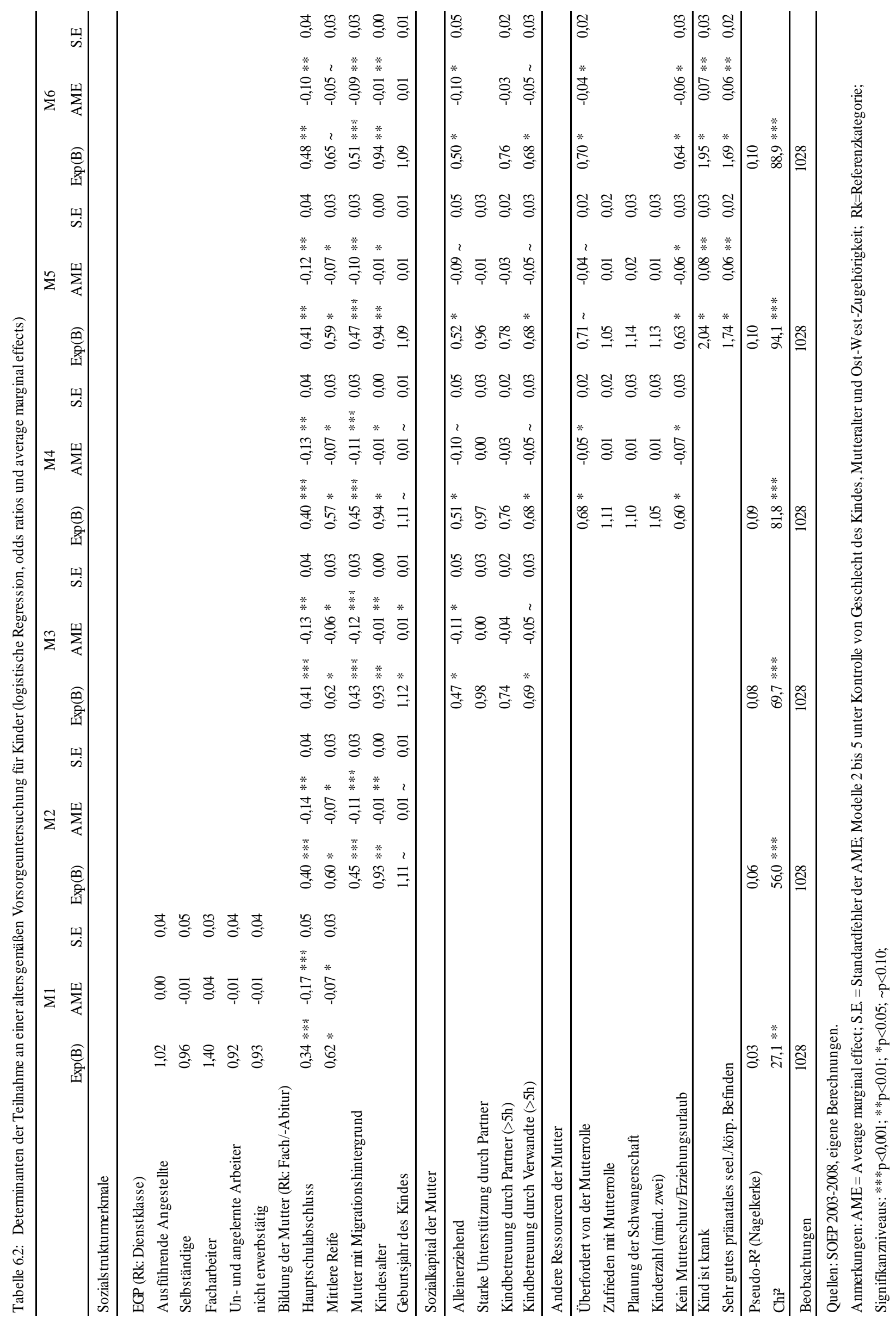


Nähern sich die odds ratios der Bildungsvariablen dem Wert 1 bzw. nähern sich die average marginal effects dem Wert 0, nimmt Sozialkapital eine zwischen der Bildung und dem Gesundheitshandeln vermittelnde Rolle ein. Wie man in Modell 3 an den unverändert starken Bildungseffekten ablesen kann, trifft dies nicht zu. Allerdings lässt sich der bereits deskriptiv ermittelte Zusammenhang zwischen Sozialkapital und dem Vorsorgehandeln auch in der multiplen Regression beobachten. Unsere erste Hypothese, nach der Kinder, die nur mit einem Elternteil aufwachsen, im Vergleich zu Kindern, die mit beiden Elternteilen aufwachsen, seltener an Vorsorgeuntersuchungen teilnehmen, wird empirisch unterstützt: Kinder alleinerziehender Mütter haben nur eine halb so große Teilnahmechance wie Kinder, die mit Mutter und Vater zusammen leben (odds ratio=0,47).

Demgegenüber lassen sich, wie deskriptiv aufgezeigt, auch Hypothesen 2 und 3, nach der positive Effekte von Unterstützung und Kinderbetreuung durch Partner und Verwandte auf das Vorsorgeverhalten erwartet werden, mit den vorliegenden Daten nicht belegen. Bemerkenswerterweise verringert sich sogar - wie schon in den Modellen in Tabelle 6.1 gezeigt die Wahrscheinlichkeit für gesundheitsbezogenes Vorsorgehandeln, wenn Verwandte in stärkerem Umfang ( $>5 \mathrm{~h}$ pro Woche) die Kindbetreuung übernehmen (odds ratio $=0,7$ ).

In Modell 4 werden schließlich das Erklärungspotential der übrigen Ressourcen der Mutter - ihres psychosozialen Wohlbefindens und ihrer zeitlichen Verfügbarkeit - geprüft. Ein gutes Zurechtkommen mit der Mutterrolle $(\mathrm{p}<0,10)$ erhöht erwartungsgemäß die Wahrscheinlichkeit der Teilnahme an den U-Untersuchungen, allerdings bleiben die Zufriedenheit mit der Mutterrolle und einer Planung der Schwangerschaft ohne signifikanten Einfluss. Die Hypothese 4 lässt sich somit nur teilweise bestätigen. Im Anschluss an Coleman (1988) wurde in der letzten Hypothese eine positive Wirkung der zeitlichen Verfügbarkeit der Mutter für gesundheitsförderliches Handeln angenommen. Die Untersuchung ergibt nur für eine der Variablen den erwarteten Effekt: Mütter, die sich nicht im Mutterschutz bzw. Erziehungsurlaub/Elternzeit befinden, gehen seltener zur Kinderkrankheitsvorsorge als ihre Vergleichsgruppe (odds ratio $=0,63$ ).

Mit Modell 5 wird zusätzlich die Gesundheit von Mutter und Kind kontrolliert. Wie bereits im Hypothesenteil angesprochen, erfolgt die Berücksichtigung dieser Variablen zur Kontrolle möglicher Endogenitätsprobleme. Ungeachtet dessen, dass sowohl die Mutter- als auch die Kindgesundheit gute Prädiktoren für das Vorsorgehandeln sind - beide Koeffizienten sind auf dem 5\%-Niveau signifikant - gibt es keine Anzeichen für einen umgekehrten Kausalzusammenhang, weil sich unter Kontrolle des kindlichen und mütterlichen Gesundheitsstatus weder die Stärke noch die Richtung der Unterstützungskoeffizienten (odds ratios, average marginal effects) ändern.

\subsection{Diskussion und Zusammenfassung}

Der vorliegende Beitrag untersuchte soziale Ungleichheiten im Gesundheitshandeln von Eltern am Beispiel der Inanspruchnahme der Vorsorgeuntersuchungen für Kinder. Im Forschungsgebiet gesundheitlicher Ungleichheiten spielen Kinder eine bedeutsame Rolle, da die 
Gesundheit im Erwachsenenalter früh geprägt wird (Richter und Hurrelmann 2007). Unser Hauptinteresse galt der Offenlegung der sozialen Mechanismen, die sich für den Zusammenhang zwischen sozialer Herkunft und dem elterlichen Gesundheitshandeln verantwortlich zeigen. Aktuelle theoretische Forschungsarbeiten schreiben dem Sozialkapitalkonzept eine bedeutsame Rolle für die Erklärung sozialer Unterschiede in der Gesundheit und Gesundheitshandeln zu. In der Bundesrepublik gibt es bisher jedoch nur wenige Studien, die die Erklärungskraft sozialer Beziehungen und die in ihnen enthaltenen Ressourcen untersuchen.

Unsere zentrale Untersuchungsfrage war daher, inwiefern soziale Unterschiede in der Inanspruchnahme der U-Untersuchungen für Kinder über die mütterliche Ausstattung mit sozialem Kapital erklärt werden kann. Die empirischen Analysen basierten auf den SOEP-Daten der Mutter-Kind-Befragung zum Säuglingsalter (Wellen 2003-2008).

Die Schätzergebnisse multipler logistischer Regressionsanalysen bestätigten, erstens, einen hochsignifikanten Einfluss der mütterlichen Bildung auf die Chance, an einer altersgemäßen Gesundheitsuntersuchung für Kinder teilzunehmen. Ein ähnlich starker Effekt ließ sich für die ethnische Herkunft beobachten. Kinder mit Migrationshintergrund haben gegenüber ihrer Vergleichsgruppe deutlich geringere Teilnahmechancen. Im Gegensatz dazu wurde für die elterliche Klassenlage, unter Kontrolle von Bildung, kein signifikanter Effekt nachgewiesen.

Zweitens unterstützen die Ergebnisse nur einen Teil unserer Hypothesen zur Verhaltenswirksamkeit von Sozialkapital. So trägt das Sozialkapital der Mutter im Hinblick auf die Unterstützung bei der Kinderbetreuung durch den Partner und die Verwandtschaft nicht entscheidend zur Vorhersage der Wahrscheinlichkeit bei, eine altersgemäße Vorsorgeuntersuchung für Kinder in Anspruch zu nehmen. Lediglich ein Sozialkapitalaspekt scheint hier von größerer Bedeutsamkeit zu sein: Lebt die Mutter mit dem Kindesvater zusammen, steigt die Teilnahmewahrscheinlichkeit. Dieser Befund weist daraufhin, dass eine gemeinsame Elternschaft die Fürsorge von Neugeborenen erleichtert.

Bemerkenswert ist darüber hinaus, dass Kinder, welche regelmäßig mehr als fünf Stunden pro Woche von Verwandten betreut werden, seltener an präventiven Gesundheitsuntersuchungen teilnehmen. Dieser Befund bedeutet in erster Linie, dass sich unsere Annahmen zur Rolle sozialer Unterstützung bei der Kindsbetreuung für das Gesundheitshandeln in einer allgemeingültigen Form nicht aufrechterhalten lassen. So kann nicht verallgemeinernd vermutet werden, dass sich eine regelmäßige Übernahme der Kinderbetreuung in einer höheren Teilnahmechance niederschlägt. Möglicherweise sind Mütter, welche in einem größeren Umfang die Kinderbetreuung an andere Familienmitglieder abgeben, aus bestimmten Gründen weniger in der Lage oder gewillt die Fürsorge für ihr Kind zu übernehmen, was sich wiederum in einer reduzierten Teilnahmewahrscheinlichkeit ausdrücken dürfte. Weiterhin gibt es empirische Evidenz für die Wirksamkeit des mütterlichen Wohlbefindens und ihrer zeitlichen Verfügbarkeit bzw. Präsenz. So haben Kinder dann signifikant bessere Teilnahmechancen, wenn Mütter im Rahmen des Mutterschutzes bzw. Erziehungsurlaubs/Elternzeit Zeit für ihre Kinder haben und den Anforderungen der Mutterrolle gewachsen sind. 
Insgesamt verdeutlichen die empirischen Ergebnisse jedoch, dass die Indikatoren für das Sozialkapital der Mütter, einschließlich ihrer weiteren - gesundheitlichen und zeitlichen Ressourcen nicht als intermediäre Größen für den Zusammenhang zwischen sozialer Herkunft und den Teilnahmechancen am Krankheitsfrüherkennungsprogramm für Kinder infrage kommen und diesen demzufolge auch nicht erklären können. Denn auch nach Kontrolle der verschiedenen Sozialkapitalien und anderen Ressourcen der Mütter reduzierte sich der Bildungseffekt - wie auch der ethnische Herkunftseffekt - kaum. Die eingangs aufgeworfene Frage, welche Mechanismen dem sozialen Gefälle im Gesundheitshandeln zugrunde liegen, kann mit der vorliegenden Forschungsarbeit also nicht beantwortet werden.

Zusammenfassend lässt sich festhalten, dass die Ergebnisse der empirischen Analyse ein eher skeptisches Bild bezüglich des Erklärungspotentials mütterlichen Sozialkapitals für das soziale Gefälle im Gesundheitsverhalten zeichnen. Vermutlich erhöht primär soziales Kapital in Paarbeziehungen die Partizipationschancen. Außerdem kommen weitere wichtige Faktoren für die Erklärung der Inanspruchnahme der U-Untersuchungen infrage, wie zum Beispiel die Kompetenzen von Müttern und Vätern zur Bewältigung der Elternrolle sowie deren zeitliche Ressourcen. Die entscheidende Rolle spielen wahrscheinlich das gesundheitskulturelle Kapital und Einstellungen der Eltern. Hier liegt auch der größte Schwachpunkt der Untersuchung, in die keine direkten Maße für das Gesundheitswissen und die Gesundheitsorientierung berücksichtigt wurden. Allerdings werden diese Faktoren indirekt durch den Bildungsabschluss der Mutter kontrolliert, welcher in den Analysen einen starken Einfluss auf das elterliche Vorsorgehandeln ausübte (Becker und Kurz 2011: 390).

Welche sozialen Prozesse zwischen der sozialen und ethnischen Herkunft auf der einen und dem Gesundheitshandeln auf der anderen Seite wirken, müssen zukünftige Arbeiten in diesem Forschungsbereich ergründen. Aus einer gesundheitspolitischen Perspektive wäre in diesem Zusammenhang die Frage aufzuwerfen, ob das in den letzten Jahren gesetzlich eingeführte Einlade- und Meldewesen (vgl. Ostner und Stolberg 2015) eine Maßnahme sein könnte, sekundäre Herkunftseffekte (jeglicher Art) in der Inanspruchnahme der Vorsorgeuntersuchungen für Kinder wirksam abzuschwächen. Mithilfe des Instruments Früherkennung könnte also ein institutioneller Beitrag geleistet werden, im Vorschulalter auftretende Entwicklungsbeeinträchtigungen früh- und somit rechtzeitig vor Beginn der Schullaufbahn auszugleichen. 
Anhang

Tabelle A 6.1: $\quad$ Überblick zur Komposition des Untersuchungssamples

\begin{tabular}{|c|c|c|c|}
\hline Variablen & Kategorien & $\mathrm{N}$ & $\begin{array}{l}\text { Prozent/ } \\
\text { Mittelwert }\end{array}$ \\
\hline \multirow[t]{2}{*}{ Teilnahme des Kindes an Vorsorgeuntersuchung } & $\mathrm{Ja}$ & 861 & 83,75 \\
\hline & Nein & 167 & 16,25 \\
\hline \multicolumn{4}{|l|}{ EGP } \\
\hline & Dienstklasse & 342 & 33,27 \\
\hline & Ausführende Angestellte & 102 & 9,92 \\
\hline & Selbstständige & 75 & 7,3 \\
\hline & Facharbeiter & 194 & 18,87 \\
\hline & Un-/angelernter Arbeiter & 122 & 11,87 \\
\hline & Nicht erwerbstätig & 137 & 13,33 \\
\hline & Missings & 56 & 5,45 \\
\hline \multicolumn{4}{|l|}{ Bildungsabschluss der Mutter } \\
\hline & (Fach-)Hochschulreife & 385 & 37,45 \\
\hline & Mittlere Reife & 392 & 38,13 \\
\hline & Haupts chulabschluss & 216 & 21,01 \\
\hline & Missings & 35 & 3,4 \\
\hline \multirow[t]{2}{*}{ Migrationshintergrund der Mutter } & Kein Migrationshintergrund & 776 & 75,49 \\
\hline & (In-)Direkter Migrationshintergrund & 252 & 24,51 \\
\hline \multirow[t]{2}{*}{ Westdeutschland } & $\mathrm{Ja}$ & 769 & 74,81 \\
\hline & Nein & 259 & 25,19 \\
\hline \multirow[t]{2}{*}{ Alter der Mutter zum Zeitpunkt der Geburt } & Metrisch & 1028 & 30,08 \\
\hline & $\begin{array}{l}\text { (S.E.) } \\
\text { Metrisch }\end{array}$ & 1028 & $\begin{array}{c}(0.174) \\
7.03\end{array}$ \\
\hline Alter des Kindes im Jahr der Erhebung & $\begin{array}{l}\text { Metrisch } \\
\text { (S.E.) }\end{array}$ & $10 \angle 8$ & $(0.117)$ \\
\hline \multirow[t]{2}{*}{ Geschlecht des Kindes } & Mädchen & 512 & 49,81 \\
\hline & Junge & 516 & 50,19 \\
\hline \multirow[t]{2}{*}{ Alleinerziehende Mutter } & $\mathrm{Ja}$ & 88 & 8,56 \\
\hline & Nein & 940 & 91,44 \\
\hline \multirow[t]{2}{*}{ Starke Unterstützung durch den Partner } & $\mathrm{Ja}$ & 788 & 76,65 \\
\hline & Nein & 240 & 23,35 \\
\hline \multirow[t]{2}{*}{ Kindbetreuung durch Partner (>5 Stunden pro Woche) } & $\mathrm{Ja}$ & 613 & 59,63 \\
\hline & Nein & 415 & 40,37 \\
\hline \multirow[t]{2}{*}{ Kindbetreuung durch Verwandte ( $>5$ Stunden pro Woche) } & $\mathrm{Ja}$ & 287 & 27,92 \\
\hline & Nein & 741 & 72,08 \\
\hline \multirow[t]{2}{*}{ Zufrieden mit der Mutterrolle } & $\mathrm{Ja}$ & 586 & 57,00 \\
\hline & Nein & 442 & 43,00 \\
\hline \multirow[t]{2}{*}{ Überfordert mit der Mutterrolle } & $\mathrm{Ja}$ & 483 & 46,98 \\
\hline & Nein & 545 & 53,02 \\
\hline \multirow[t]{2}{*}{ Planung der Schwangerschaft } & $\mathrm{Ja}$ & 723 & 70,33 \\
\hline & Nein & 305 & 29,67 \\
\hline \multirow[t]{3}{*}{ Geschwisteranzahl } & 0 & 577 & 56,13 \\
\hline & 1 & 286 & 27,82 \\
\hline & 2 und mehr & 165 & 16,05 \\
\hline \multirow[t]{2}{*}{ Mutterschutz/Mutterschaftsurlaub/Elternzeit } & $\mathrm{Ja}$ & 811 & 78,89 \\
\hline & Nein & 217 & 21,11 \\
\hline \multirow[t]{2}{*}{ Kind ist krank } & $\mathrm{Ja}$ & 152 & 14,79 \\
\hline & Nein & 876 & 85,21 \\
\hline \multirow[t]{2}{*}{ Sehr gutes pränatales physisches/seelisches Befinden } & $\mathrm{Ja}$ & 335 & 32,59 \\
\hline & Nein & 693 & 67,41 \\
\hline
\end{tabular}

Anmerkung: Quelle: SOEP 2003-2008, eigene Berechnungen. 


\section{Lebensanfängliche Gesundheitsungleichheiten und ihre Bildungsfolgen}

\subsection{Einleitung ${ }^{58}$}

Hochmotiviert durch den Wunsch nach sozialen Aufstieg streben Migrantennachkommen typischerweise höherwertige Bildungsabschlüsse an (Nauck 1994; Kurz und Paulus 2008), erwerben diese aufgrund niedriger Leistungen jedoch relativ selten (Gresch und Becker 2010). Mit der Schwangerschaft verbundene schulerfolgsrelevante Bedingungen verdienen in diesem Kontext besondere Beachtung (Becker und Biedinger 2006: 660; Jackson 2013: 13; Kristen 2008: 231, 247). Im vorliegenden Beitrag steht daher die These auf dem empirischen Prüfstand, dass schulisches Gelingen oder Misslingen nicht allein auf inner- und außerfamiliäre Lernprozesse, sondern teils auf vorgeburtliche Einflüsse zurückzuführen ist.

Für Deutschland liegen zu dieser Thematik mangels geeigneter Daten nur wenige Forschungsarbeiten vor (Jäkel et al. 2013). Ob sich Frauen mit und ohne ausländische Wurzeln mit Blick auf das Geburtsergebnis systematisch unterscheiden, kann noch nicht abschließend beurteilt werden. Erste Studien belegen für Migrantennachkommen geringere Chancen auf einen gesunden Start ins Leben (Bollini et al. 2008; Razum et al. 2011). Der Forschungsstand ist aber sehr brüchig, vor allem, wenn nach einzelnen Migrantengruppen unterschieden wird. Wie Surveystudien zeigen (RKI 2008; Milewski und Peters 2014), entbinden türkischstämmige Frauen häufiger zu früh, d. h. vor Vollendung der 37. Schwangerschaftswoche (Frühgeburt). Türkischstämmige Neugeborene sind auch häufiger leichter als 2500 Gramm (niedriges Geburtsgewicht) (Milewski und Peters 2014). Demgegenüber belegen andere Studien für diese Herkunftsgruppe keine bedeutsamen Geburtsdifferenzen (Reime et al. 2006; David et al. 2006). Inkonsistent ist auch die Evidenz für Spätaussiedlerinnen. Zwar wird zum Teil eine höhere Frühgeburtsprävalenz beobachtet, allerdings sind Neugeborene im Mittel sogar schwerer zur Geburt als deutsche Neugeborene (Milewski und Peters 2014).

$\mathrm{Ob}$ eine Auseinandersetzung mit schwangerschaftsbezogenen Differenzen dazu beiträgt, ethnische Bildungsungleichheiten besser zu verstehen, ist noch ein Forschungsdesiderat. Da frühe Nachteile vermutlich über die Zeit zunehmen (Erhart et al. 2008: 334) und damit den Bildungswettbewerb vorstrukturieren, ist es unter mehreren Aspekten wichtig, mehr über das Zustandekommen ungleicher Startbedingungen und den damit verknüpften schulischen Folgen zu erfahren: Soziologisch aus Erkenntnisinteresse, sozialpolitisch aus Gründen der Prävention, Kompensation und Chancengleichheit sowie volkswirtschaftlich zur Vermeidung hoher Hospitalisierungskosten und zur optimalen Bildungsausschöpfung. Das Thema berührt des Weiteren die in Wissenschaft und Politik geführte Diskussion zur bildungsbezogenen Inklusion (Cramer und Harant 2014). Um diese ist es in Deutschland zum jetzigen Zeitpunkt eher schlecht bestellt. Auf Lernbedürfnisse von Risikokindern, wie Frühgeborenen, ist das Regelschulsystem noch nicht adäquat eingestellt. Es droht früh das schulische Abseits (Wolke

\footnotetext{
${ }^{58}$ Dieser Beitrag basiert auf einem unveröffentlichten Manuskript (Becker 2016).
} 
und Meyer 2000: 113): Spiegelbildlich zu Migrantenkindern schlagen sich Frühgeborene suboptimal im Bildungssystem, wie relativ hohe Raten der Sonderbeschulung und Klassenwiederholungen, niedrige Leistungen und Noten sowie eine geringe Repräsentanz in weiterführenden Schulformen belegen. Es stellt sich somit folgende Forschungsfrage: Ist Bildung mit in die Wiege gelegt? Lassen sich Differenzen zwischen Kindern mit und ohne ausländische Wurzeln an der Schwelle zur Grundschule, im Primarbereich und Sekundarstufe über schwangerschaftsbezogene Bedingungen erklären?

In diesem Zusammenhang wird weiterhin untersucht, ob sich für Kinder aus Migrantenfamilien überhaupt entwicklungsbezogene Nachteile zum Beginn des Lebens ergeben. Zu dieser Teilfrage besteht gegenwärtig ein großes Wissensdefizit. Primäres Ziel ist herauszufinden, ob in multivariaten Analysen nach Berücksichtigung prä- und postnataler Größen ethnische Bildungsdisparitäten fortbestehen. Diese Frage stellt sich besonders für türkischstämmige Kinder, deren Bildungsnachteile sich oftmals nur partiell über sozioökonomische und sprachkulturelle Pfade aufklären lassen (Kristen 2008). Für die theoretische Rahmung der Fragestellung lohnt ein ressourcentheoretischer Zugang. Gesundheit und Bildung werden als soziale Kategorien bzw. Kapital aufgefasst, dessen Erwerb primär von den elterlichen Möglichkeiten zur Förderung des Nachwuchses abhängt (Bourdieu 1983; Bradley und Corwyn 2002). Die Opportunitäten zur Entwicklungsförderung sind für in Deutschland lebende Migrantenfamilien aufgrund ihrer relativen sozioökonomischen Schlechterstellung eher begrenzt. Hieraus können bereits bei Neugeborenen bedeutsame Entwicklungsnachteile resultieren (Corman et al. 1987), welche sich - bei ausbleibender Kompensation - über den frühkindlichen Lebensverlauf in Kompetenzrückständen manifestieren und so die Chancen auf eine reguläre Einschulung, auf gute Schulnoten und auf den Besuch einer weiterführenden Schulform schmälern.

Die Hypothesenüberprüfung stellt besondere Datenanforderungen, denen der Kinder-undJugendgesundheitssurvey (KiGGS 2003-2006) gerecht wird. Einerseits erlaubt die Größe der Stichprobe die Unterscheidung der beiden größten Migrantengruppen - Zugewanderte türkischer und ethnisch-deutscher Herkunft. Andererseits wurden detaillierte Angaben zu Schwangerschaft, entwicklungsbezogenen Investitionen (Substanzkonsum während der Schwangerschaft, Vorsorgehandeln, Kindergartenbesuch) und sozialen Herkunft erhoben. Schulerfolg wird anhand von drei Kriterien gemessen: Dem fristgerechten bzw. vorzeitigen Überschreiten der Schuleingangsschwelle (vs. Rückstellung vom Schulbesuch), anhand der Grundschulnoten in Mathematik und Deutsch sowie des Besuchs eines weiterführenden Sekundarschultyps (Realschule/Gymnasium/Gesamtschule vs. Haupt-/Förderschule).

Der Beitrag gliedert sich wie folgt: Nach den theoretischen Überlegungen in Abschnitt 2 werden Daten und Methodik (Abschnitt 3) vorgestellt. Daran schließt sich der Ergebnisteil an (Abschnitt 4). Zunächst wird der Zusammenhang zwischen Elternhaus (Sozialschicht, Risikohandeln) und Schwangerschaftsergebnis (Schwangerschaftsdauer, Geburtsgewicht, postnatale Gesundheitsprobleme) untersucht. Beide Merkmalsbündel werden sodann mit anderen schulrelevanten Größen (Frühinvestitionen, Familiensprache) zur Vorhersage der Rückstellung vom Schulbesuch herangezogen. Schließlich werden alle Modellgrößen für die Analyse der 
Schulnoten und des Schulbesuchs zusammengeführt. In Abschnitt 5 erfolgt die Zusammenfassung und Diskussion der zentralen Ergebnisse.

\subsection{Zur Erklärung ethnischer Gesundheits- und Bildungsunterschiede}

Anthropologisch betrachtet ist der menschliche Nachwuchs existenziell (Esser 1999: 162) auf das elterliche Wohlwollen und Wohlergehen angewiesen. Für eine optimale pränatale (Corman et al. 1987) und postnatale Entwicklung (Hurrelmann 1988) sowie für den späteren Schulerfolg ist die aktive Mitarbeit der Eltern unabdingbar (Bourdieu 1983). Differenzen im Gesundheits- und Bildungskapital basieren in diesem Kontext auf unterschiedlichem Handeln im Hinblick auf Beginn, Art, Umfang und Effizienz elterlicher Investitionen. Ethnische Investitionsunterschiede werden also als Ergebnis soziokultureller und migrationsbedingter Restriktionen und deren wechselseitiges Zusammenspiel verstanden (Kristen und Granato 2007).

Abbildung 7.1 skizziert zum Überblick das Erklärungsmodell. Demnach hängen gute Noten und der Besuch höherwertiger Bildungseinrichtungen primär vom Erwerb schulrelevanter Kompetenzen $a b$, deren Besitz an der Transition zur Grundschule schulärztlich im Rahmen einer Schulfähigkeitsuntersuchung festgestellt wird (Becker und Biedinger 2006). Die Kompetenzaneignung erfolgt dabei in erster Linie im Elternhaus und in zweiter Linie im Kinderbetreuungssystem. Daneben kommt vorgeburtlichen Entwicklungsumständen und Frühinvestitionen, so die Grundannahme, eine schulerfolgsprägende Rolle zu.

\section{Abbildung 7.1: Erklärungsschema}

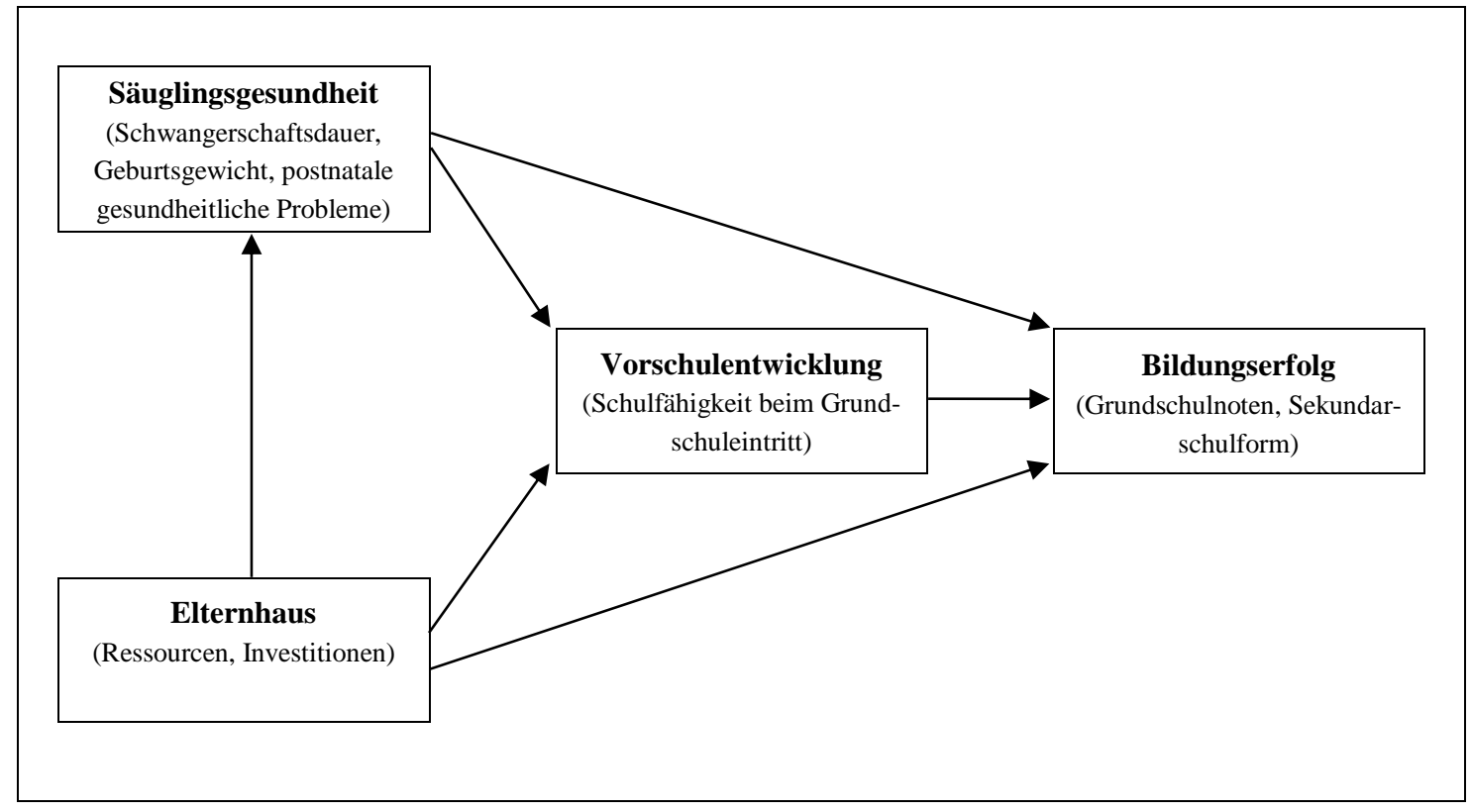

Anmerkung: Modifizierte Darstellung nach Becker und Biedinger (2006: 666)

Zur Erklärung ethnischer Differenzen im Geburtsergebnis ist es - wie für das Verständnis ethnischer Bildungsungleichheiten - hilfreich zwischen einem allgemeinen (auf soziale Herkunftseffekte abstellenden) Argumentationsstrang und einem Zugang zu unterscheiden, der 
spezifisch mit der ethnischen Herkunft und Migration verknüpfte Einflüsse näher in den Blick nimmt (Razum et al. 2011; Schenk 2007; Spallek et al. 2010).

\subsubsection{Zur Entstehung ethnischer Ungleichheit zum Lebensbeginn}

Die Prägung des menschlichen Fötus vollzieht sich stärker im Verborgenen als die Erziehung. Soziale Umstände können daher, mitvermittelt über eine gesundheitsriskante Lebensweise, relativ ungefiltert auf die intrauterine Entwicklung wirken.

Eine sozioökonomische Besserstellung geht mit Gesundheitsprivilegien und daher mit differentem Reproduktionserfolg einher (Mielck 2005; Siegrist 2005). Während der Schwangerschaft kann je nach sozialer Zugehörigkeit auf verschiedenstem Wege mehr oder weniger bewusst gesundheitliche Erträge erzielt werden. Sozial Bessergestellte leben in ökologisch privilegierten Wohngegenden mit geringer Exposition zu Umweltgiften, was die Wahrscheinlichkeit für perinatale Komplikationen (Frühgeburt) verringert (Porter et al. 2014). Hierfür ist indirekt das Einkommen bedeutsam. Ökonomisches Kapital hat zudem eine stresstheoretische Relevanz (Collatz et al. 1979). Die Familiengründung/-erweiterung bedeutet langfristig finanzielle Einschränkungen, die besonders von Schwangeren mit geringem Einkommen als Belastung empfunden werden (Bründel und Hurrelmann 1996: 22). Da formal niedrig gebildete Frauen häufig mit ähnlich niedrig gebildeten (und somit relativ einkommensschwachen) Partnern zusammenleben, sind die gesundheitlich wertvollen Unterstützungsressourcen ungleich verteilt (Jungbauer-Gans 2002).

Für die Lebensführung spielt das Bildungskapital schwangerer Frauen eine Schlüsselrolle (Currie und Moretti 2003). Formal höher Gebildete verfügen über eine stärker ausgeprägte Kompetenz (Wissen, Selbstwirksamkeitserwartungen), Gesundheit im Alltag effizient herzustellen (Mirowsky und Ross 2003, 2007). Je höher die Bildung, desto geringer die Wahrscheinlichkeit, das Wohl des Ungeborenen mehr oder weniger bewusst zu gefährden. So begünstigen der Konsum von Tabak, Alkohol, Medizin oder eine Mangelernährung Fehlbildungen, Frühgeburten und niedriges Geburtsgewicht (Voigt et al. 2009).

Mit steigendem Bildungsgrad wird also früher und kontinuierlicher in den Nachwuchs investiert: Die Schwangerschaft wird häufiger geplant, das Risikoverhalten antizipatorisch (meist vor der Konzeption) angepasst sowie Schwangerenvorsorge regelmäßiger genutzt (Raum et al. 2001). Ebenso sind Kontrolle und Durchsetzung normativer Erwartungen an eine gesunde Lebensführung mit steigendem Bildungsgrad des Partners wahrscheinlicher. Auch können über Passivrauchen, was verstärkt in unteren Bildungsschichten auftritt, entwicklungsschädigende Substanzen zum Kind gelangen (van den Berg et al. 2012).

Neben sozialen Umständen während der Schwangerschaft können mit der Migration und ethnischen Herkunft zusammenhängende Bedingungen die Entwicklung des menschlichen Fötus prägen. Zuzuwandern heißt, sich in einem neuen Gesundheitskontext zu orientieren. Da für das Gesundheitshandeln im Herkunftsland wichtige Kapitalien im Aufnahmeland an Wert verlieren, gilt es unter Ressourcenaufwand sozialrechtliche Versorgungsofferten und Versorgungsansprüche in Erfahrung zu bringen (Pränataldiagnostik, Kolleck et al. 1979). Ferner 
müssen die Landessprache erlernt und Institutionenvertrauen aufgebaut werden (Fassaert et al. 2009). Für das Geburtsergebnis von besonderer Relevanz ist die Teilnahme an der Schwangerenvorsorge. Allerdings werden Frauen mit hohem Präventionsbedarf, besonders (niedrig gebildete) Frauen mit ausländischen Wurzeln, trotz eines kostenlosen Schwangerenvorsorgeangebots seltener präventiv erreicht (Collatz et al. 1983; Razum et al. 2011). Schwangere mit familiärer Zuwanderungsbiographie suchen zum Teil erst bei Komplikationen (z. B. Blutungen) ärztlichen Rat (Fassaert et al. 2009).

Allgemein liegen Vorsorgebarrieren je nach Herkunftskontext und tradierten Einstellungen unterschiedlich hoch (Andersen 1995; Phillips et al. 1998; Scheppers et al. 2006). Ähneln sich Vorsorgekontexte in Herkunfts- und Zielland, sollte die Vorsorgeneigung stärker, mit stärkerem Systemkontrast schwächer ausgeprägt sein. Kulturell differentes Denken über Gesundheit kann den selbstgesteuerten Zugang zum Versorgungssystem verengen: Wird Gesundheit als gottgegeben wahr- und als Schicksal angenommen (fatalistischer Habitus, Hartung et al. 2010; Spallek et al. 2010), ergibt alltagsintegrierte Gesundheitsförderung und Prävention subjektiv wenig Sinn. ${ }^{59}$

Angewandt auf die beiden Migrantengruppen lässt sich somit argumentieren: Schwangere mit (türkischem) Migrationshintergrund haben ressourcenbedingt und da ökonomische, kulturelle und soziale Kapitalien dem gesundheitlichen Handlungsspielraum Grenzen setzen (Abel 2008; Williams 1995), auch verhaltensbedingt vergleichsweise hohe Risiken für Komplikationen. Empirische Evidenzen unterstützen die These differentieller Lebensführungsmuster. In mediterranen Herkunftsländern oder in Zentral- und Osteuropa geborene Frauen gehen hierzulande seltener zu den Schwangerenvorsorgeuntersuchungen (Reime et al. 2009). Allerdings rauchen Spätaussiedlerinnen während der Schwangerschaft seltener als türkische Frauen (RKI 2008; Milewski und Peters 2014). Für türkischstämmige Schwangere lässt sich somit ressourcen- und verhaltensbedingt ein besonders hoher Präventionsbedarf ableiten. Dieser bleibt infolge (spezifisch-)kultureller Distanzen zur Schwangerenvorsorge allerdings eher ungedeckt. So wandern Türkinnen aus einem (vor allem im östlichen Landesteil relativ) strukturschwachen Präventionskontext zu, in dem traditionelle Präventionseinstellungen vorherrschen (Erci 2003). ${ }^{60}$ Die Mehrheit der Schwangeren nutzt die gesetzlich verankerte und kostenlose Vorsorge in der Türkei unregelmäßig, spät oder (zu $32 \%$ ) überhaupt nicht (Ergöçmen 1999). Im Gegensatz wandern die relativ bildungsstarken Spätaussiedlerinnen mehrheitlich aus osteuropäischen Ländern zu, in denen die Versorgungsstruktur/-kultur eher westlichen Standards entspricht. So liegen die Anteile der Nicht-Nutzerinnen in europäischen Ländern zwischen $0,5 \%$ und 2,6\% (Delvaux und Buekens 1999) und niedriger als in der Türkei (Erci 2003: 269).

\footnotetext{
59 Ferner entscheiden nicht in allen Gesellschaften Frauen über Art und Umfang schwangerschaftsbezogener Investitionen, sondern teils Partner und die Familie (Reime et al. 2009: 1281).

${ }^{60}$ Schlechte Erreichbarkeit, familiäre Verpflichtungen, ungeplante Schwangerschaften, negative Einstellungen zu Prävention und niedrige Bildung sind Teilnahmebarrieren (Cindoglu und Sirkeci 2001; Erci 2003).
} 
Aus den bisherigen Überlegungen leiten sich die ersten zwei Hypothesen ab: Ethnische Unterschiede in den Geburtsergebnissen sollten zu Ungunsten von Migrantinnen ausfallen, wobei türkischstämmige Kinder, bemessen an der Dauer der Schwangerschaft, Geburtsgewicht und postnatalen Gesundheitsproblemen relativ starke Nachteile, Kinder aus sozial privilegierteren Spätaussiedlerfamilien hingegen weniger ausgeprägte Nachteile haben (Hypothese 1). Beide Gruppen unterscheiden sich gegenüber Nicht-Migranten mit Blick auf die Lebensführung (Rauchen) und auf das Präventivhandeln (Hypothese 2).

\subsubsection{Zur Entstehung ethnischer Ungleichheit im Kompetenz- und Bildungserwerb}

Analog zur Erklärung lebensanfänglicher Entwicklungsdisparitäten tragen mit der sozialen Herkunft sowie spezifisch mit der Migration (Wissen zu/Vertrautheit mit Bildungssystem) und ethnischen Zugehörigkeit (Familiensprache; Einstellungen zu Frühförderung und Prävention) verbundene Merkmale zu ethnischer Differenz beim Humankapitalerwerb bei. Im Allgemeinen gilt: Je privilegierter insbesondere die kulturelle Ressourcenausstattung, desto lernanregender die Zeit „im Schoß der Familie“ (Bourdieu 1976: 225) und desto vorteilhafter der Kompetenzerwerb im Vorschulalter und desto ausgeprägter die für ein erfolgreiches Durchlaufen der Grundschule wichtige Schulfähigkeit (Tuppat und Becker 2014). Einfluss auf den Schulerfolg können Eltern auch unter Aufwand von persönlicher Zeit (Hausaufgabenhilfe) und ökonomischen Kapital (Geld für Nachhilfe und Lernmöbel; ausreichend Wohnplatz für ungestörtes Lernen) nehmen (Dollmann 2010).

Spezifisch erfolgsrelevant im Unterricht ist für Migrantenkinder die Familiensprache. Zugewanderte Familien unterscheiden sich in der Beherrschung der Aufnahmelandsprache sowie der Motivation, diese zu erlernen und im Familienalltag konsequent anzuwenden. Je nach ethnischer Zugehörigkeit leiten sich hieraus differentielle Bildungserträge ab (Becker und Biedinger 2006; Kristen 2008). So können Migranteneltern, welche die Sprache des Ziellandes nicht beherrschen und/oder das Bildungssystem nicht selbst durchlaufen sind, nur begrenzt in schulischen Belangen unterstützten. Damit fällt es auch schwerer sich an Übergängen strategisch zu verhalten.

Für beide Migrantengruppen ist nun anzunehmen: Für türkischstämmige Kinder, deren Eltern im Mittel über einen formal niedrigen Bildungsgrad verfügen, sind vorschulische Anregungsimpulse (Jäkel et al. 2013) und häusliche Sprach-/Förderopportunitäten stärker begrenzt als bei Spätaussiedlernachkommen, deren Eltern formal ähnlich gut gebildet sind wie Deutsche (Becker und Biedinger 2006). Gegenüber türkischstämmigen Kindern haben Spätaussiedlerkinder auch das häusliche Lernprivileg, mit aufnahmelandspezifischem Spracherbe aufzuwachsen (Strobel und Kristen 2015), was die Sprachkompetenz und die Aneignung kulturellen Vorwissens fördert (Gresch und Becker 2010: 185). Hieraus leitet sich folgende Erwartung ab: Ungleiche familiäre Lernausgangsbedingungen mitbedingen ethnische Bildungsdifferenzen (Hypothese 3).

Neben häuslichen Lernanregungen sind außerfamiliäre Lernkontexte (Kristen 2008: 233) sowie das Präventivhandeln (Rosenkötter et al. 2012) kompetenzrelevant. Eine effiziente 
Strategie ist die Nutzung der formellen Kinderbetreuung (Kratzmann und Schneider 2009), was den Erwerb schulrelevanter Sprach- und Verhaltenskompetenzen fördert. Besonders relevant ist ein früher Betreuungsbeginn: Eine kurze Betreuungsdauer (von nur 2 Jahren) geht zum Schulbeginn (Berthold 2008: 26) und am Primarstufenende mit Kompetenzrückständen einher (Autorengruppe Bildungsberichterstattung 2012: 51). Damit sinken die Chancen auf eine Regeleinschulung, gute Noten und eine aussichtsreiche Sekundarschulpositionierung. Höhergebildete Eltern ermöglichen den Besuch qualitativ guter Kindertageseinrichtungen aus Gründen des Statuserhalts (Kratzmann und Schneider 2009: 8), aber auch zum Wiedereinstieg in den Beruf, zu einem früheren Lebensalter. Allerdings können sich dies bildungsnahe Eltern finanziell auch eher leisten (Schröder et al. 2015) und finden regional günstigere Opportunitäten vor (Becker und Biedinger 2006). Das Gros der Dreijährigen ist in Tagesstätten präsent, im U3-Bereich sind Migrantenkinder aber immer noch seltener vertreten (Autorengruppe Bildungsberichterstattung 2012: 65). Hierfür spielen die geringe formale Bildung, seltenere Erwerbstätigkeit sowie vermutlich auch Präferenzen für eine spätere statt frühe Betreuung eine Rolle (Peter und Spieß 2015).

Unterrepräsentiert sind Migrantenkinder auch im Früherkennungsprogramm (Lampert et al. 2005: 104). Im Rahmen der sogenannten U-Untersuchungen können Kinder in bestimmten Intervallen, beginnend mit der Geburt, auf eine altersgerechte Entwicklung kostenlos ärztlich untersucht werden. Wie beim Besuch einer Kindertagesstätte verhalten sich Eltern bei der Nutzung des gesetzlich verankerten Programms vermutlich strategisch. So ermöglicht eine lückenlose U-Teilnahme, etwaige Kompetenzdefizite früh zu erkennen und noch vor Grundschulbeginn zu beheben. Kinder mit ausgelassener U9 erhalten in Schuleingangsuntersuchungen häufiger mindestens eine abklärungsbedürftige Diagnose gestellt (Meyer-Nürnberger 2002: 863). Damit steigt das Risiko einer Rückstellung vom Schulbesuch (Rosenkötter et al. 2012). Womöglich wird auch die individuelle Bewältigung schulalltäglicher Anforderungen (fehlende Ausdauer/Konzentration) unwahrscheinlicher.

Beide Migrantengruppen nutzen formelle Angebote zur Frühbildung und Früherkennung relativ selten. Da der alltagsintegrierte Zweitspracherwerb und der Umgang mit anderen Kindern in Kindertagesstätten sowie eine kontinuierliche Präventionsaktivität bedeutsam sind für den Schuleintritt und Schulerfolg, müssten ethnische Bildungsdisparitäten hierüber partiell vermittelt sein (Hypothese 4).

Da Rückstellungen vom Schulbesuch beim nachträglichen Erwerb der Schulfähigkeit in Kritik stehen (Berthold 2008: 25) und diese Auslesepraxis vermutlich auch die Weichen in das untere Leistungs-/Bildungssegment voreinstellt, kann nicht ausgeschlossen werden, dass die den Klassenverband homogenisierende Selektionspraxis der Schule beim Eintritt in die Primarstufe (Instrument der Zurückstellung) vor allem zu Ungunsten der Kinder aus (türkischen) Migrantenfamilien wirkt (Gomolla und Radtke 2002). Rückstellungen vom Schulbesuch könnten somit Noten- und Positionsnachteile mitbedingen (Hypothese 5). 


\subsubsection{Schulische Konsequenzen lebensanfänglicher Entwicklungsnachteile}

Individuelle und familiäre Merkmale haben in stratifizierten Bildungssystemen mit ausgeprägter Selektions- und Allokationsfunktion besondere Relevanz (Turner 1960). Die Aufteilung der Primarschülerschaft auf die Sekundarschulen erfolgt einerseits auf Basis der erzielten Schulnoten. Andererseits lassen sich Lehrkräfte von leistungsfremden Kriterien leiten, wie dem Unterstützungspotenzial der Familie und dem Lern- und Sozialverhalten (Konzentration, Aufmerksamkeit und Disziplin) (Ditton 2008: 253). Schulrelevante Kompetenzen sind aber nicht unabhängig von schwangerschaftsbezogenen Faktoren (De Jong et al. 2012; Steinhausen et al. 2000). Nach Bourdieu ist der Bildungserwerb geprägt von den ,ersten“ Aneignungsumständen, welche „mehr [manifeste] oder weniger sichtbare [latente] Spuren [hinterlassen]“ (1983: 187). Entwicklungsbiologisch betrachtet fallen die ersten Transmissionsbedingungen in die Zeit der Schwangerschaft, in welcher der Grundstein für gesundheitliche (Erhart et al. 2008: 340; Ben-Shlomo und Kuh 2002; Cable 2015) und vermutlich auch für schulische Leistungs- und Positionsprobleme gelegt wird (Jackson 2013). Zu früh oder mit niedrigem Geburtsgewicht Geborene haben häufiger organische Reifeverzögerungen und -störungen, welche Lernprozesse ,in Umfang und Tempo nachhaltig“ beeinträchtigen können (Siegrist 2005: 190). Aufmerksam und konzentriert dem Unterricht zu folgen, Aufgaben richtig zu lösen und gute Leistungen zu erzielen, fällt diesen Kindern deutlich schwerer (Johnson et al. 2015; Lipkind et al. 2012; Poets et al. 2012). Kognitive Fähigkeiten werden zudem durch Substanzkonsum während der Schwangerschaft beeinträchtigt (Steinhausen 2000).

Unter der Bedingung zwischen ethnischen Gruppen variierender Lebensbedingungen und Lebensweisen während der Schwangerschaft ist anzunehmen, dass Bildungsungleichheiten teils über perinatale Komplikationen (Frühgeburt), gesundheitsriskantes Handeln (Rauchen während der Schwangerschaft, geringe Vorsorgeneigung) und nach der Geburt auftretende Gesundheitsprobleme vermittelt sind (Hypothese 6). Da Nachteile zum Lebensbeginn mit dem Erwerb schul- und lernrelevanter Kompetenzen verknüpft sind, ist ferner zu erwarten, dass die schlechteren Schulerfolgschancen von Migrantennachkommen auch Ausdruck von Schwierigkeiten bei der Bewältigung des Schulalltags sind (Hypothese 7).

Ergänzend hierzu kommt ein institutioneller Erklärungsmechanismus für die schulischen Nachteile von Migrantenkindern infrage, da Lehrkräfte Ungleichheitsphänomenen relativ „blind“ gegenüberstehen (Ditton 2008: 253). Das deutsche Regelschulsystem ist auf kulturelle (Diefenbach 2011) und gesundheitliche Heterogenität (Wolke und Meyer 2000) nicht adäquat eingestellt - Lehrkräften fehlt es an inklusionspädagogischer Erfahrung, Qualifikation und Unterstützung (Werning 2014; Johnson et al. 2015). Verhalten sich nun Schüler im Sinne obiger Kriterien abweichend (Aufmerksamkeit), könnten Lehrer das als schulisches Desinteresse, mangelnden Bildungswillen oder fehlende akademische Eignung auslegen, über selektive Notengebung und Empfehlungen sanktionieren und mit der meritokratischen Leistungsidee legitimieren: Verhaltensauffällige Kinder hätten dann die gymnasiale Laufbahn und gute Noten weniger verdient als verhaltenskonforme Kinder. Ethnische Bildungsungleichheiten 
könnten also auch auf eine unzureichende Erfüllung schulischer Lern- und Verhaltensanforderungen zurückzuführen sein (Hypothese 8).

Zwar erzielen auch Risikokinder Schulerfolge, hierfür bedarf es allerdings oftmals einer Individualförderung, die Mehrkind- und Migrantenfamilien ressourcenbedingt selten selbst leisten können (Jäkel et al. 2013). Unteren Sozialschichten gelingt es dabei in geringerem Maße schwangerschaftsbezogene Entwicklungsnachteile auszugleichen (Arpi und Ferrari 2013; Larroque et al. 2011). In türkischen Familien gestaltet sich die Kompensation früher Nachteile besonders schwierig, da in der Regel mehrere Kinder um relativ knappe soziale Ressourcen konkurrieren, während in Spätaussiedlerfamilien weniger Kinder unter sozial günstigeren Umständen aufwachsen (Becker und Biedinger 2006: 672). Manifestierungen kognitiver Nachteile sind also bei türkischstämmigen Kindern wahrscheinlicher, zumal diese später in außerfamiliären Lernkontexten präsent sind und den kompensatorisch wirkenden UUntersuchungen eher fernbleiben. Entsprechend prägnant sollten somit die Noten- und Positionierungsnachteile dieser Gruppe ausfallen.

\subsection{Datenbasis, Variablenkonstruktion und methodisches Vorgehen}

Für die Analysen werden die Daten des Kinder- und Jugendgesundheitssurvey (KiGGS 20032006) verwendet. Der Survey hat primär Gesundheit (Kurth 2007), nicht aber Bildung im Blick. Hieraus resultieren besondere Analysepotentiale, aber auch Restriktionen. Vorteilhaft ist die Erhebung vor- und nachgeburtlicher entwicklungs- und leistungsrelevanter Bedingungen, wie Risikohandeln, Schwangerschaftskomplikationen, Vorsorgehandeln und Besuch formeller Bildungskontexte (Kindertagesstätte). Indikatoren zu Schulerfolg (Grundschulnoten in Deutsch und Mathematik, Bildungsbeteiligung) und erfolgsrelevanten Faktoren (Schulfähigkeit, Bewältigung des Schulalltags, Sozialverhalten) liegen auch vor. Von Nachteil ist, dass für die Schulbesuchsanalysen keine Informationen zu den Grundschulnoten zur Verfügung stehen. Über das Einschulungsergebnis kann aber das Leistungspotenzial am Anfang der Grundschule näherungsweise erfasst werden. Auch liegen Elternangaben zur individuellen Funktionsfähigkeit im Schulalltag (Kindl-Skala) ${ }^{61}$ und das Sozialverhalten (unauffällig, grenzwertig, auffällig) vor. Beide Konstrukte gehen als unabhängige Variable in die Schulerfolgsanalysen ein. Wünschenswert, aber nicht im Erhebungsprogramm enthalten, wäre zudem eine Angabe zur Lehrerempfehlung gewesen.

Das zentrale Kriterium im Beitrag ist der Schulerfolg. Die Grundschulnoten in Deutsch und Mathematik (Noten wurden transformiert) sowie der Sekundarschulbesuch (Haupt-/Förderschule vs. Realschule/Gymnasium/Gesamtschule) werden als abhängige Vari-

\footnotetext{
${ }^{61}$ Die Kindle-Skala (Wertebereich: 0, ., 100; höhere Werte zeigen geringere Schulprobleme an) basiert auf 5 Items: „hat mein Kind die Schulaufgaben gut geschafft“, „,hat meinem Kind der Unterricht Spaß gemacht“, „hat mein Kind sich Sorgen um seine Zukunft gemacht“, ,hat mein Kind Angst vor schlechten Noten“.
} 
ablen verwendet. ${ }^{62}$ Die Analysen zu den Leistungen $(\mathrm{N}=2014)$ basieren auf der Schülerschaft der Klassenstufen drei bis vier (bzw. fünf/sechs in Ländern mit späterem Bildungsübergang). Die Schulbesuchsanalysen beschränken sich auf Kinder der fünften bis neunten Klassenstufe (N=3850). Die Analysen zum Eintritt in die Grundschule beziehen sich auf alle Schulkinder. Die Schulfähigkeit wird gemessen über das Einschulungsergebnis (Rückstellung vs. fristgerechte/vorzeitige Einschulung) und dient, wie die Ausgangslage nach der Geburt, sowohl als abhängige wie erklärende Variable (vgl. Abbildung 7.1). Die gesundheitliche Ausgangslage wird bestimmt über die Schwangerschaftsdauer (Geburt vor Vollendung der 37. SSW: früh Geborene, 37-38. SSW: frühe Termingeborene, 39. SSW und mehr: Termingeborene $)^{63}$, Geburtsgewicht (metrisch) und postnatale Gesundheitsprobleme (dichotom). ${ }^{64}$ Das Risikohandeln wird über den Konsum von Tabak (nein, ab und zu, regelmäßig) während der Schwangerschaft erfasst. Ein Migrationshintergrund liegt vor, wenn beide Eltern im Ausland geboren sind (vgl. für ein ähnliches Vorgehen Kristen und Dollmann 2010: 135). Kinder mit in Deutschland geborenen Eltern haben keinen Migrationshintergrund, Kinder mit in der Türkei geborenen Eltern einen türkischen, Kinder ethnisch-deutscher im Ausland geborener Eltern einen Spätaussiedlerhintergrund. Alle anderen Kinder mit zwei im Ausland geborenen Eltern werden den „Sonstigen“ zugeordnet. In diese Gruppe fallen auch Kinder mit in Deutschland geborenen Eltern, die keine deutsche Staatsangehörigkeit besitzen oder Zuhause nicht Deutsch sprechen. Der Generationenstatus wird aufgrund geringer Fallzahlen nicht unterschieden. Die Abbildung der sozialen Herkunft erfolgt über eine dreikategoriale Variable auf Basis des Schichtindexes nach Winkler ${ }^{65}$ (niedrig, mittel, hoch) und die Wohnraumgröße (Quadratmeter pro Person). Kulturelle Besonderheiten, wie die Exposition zur deutschen

\footnotetext{
${ }^{62}$ In Anlehnung an Solga und Wagner (2008: 199) wird zwischen dem Hauptschulbesuch und den weiterführenden Schulformen (Realschule, Gesamtschule, Gymnasium) unterschieden, da im Zuge der Bildungsexpansion Migrantenkinder überproportional an der zunehmend verdrängten Hauptschule zurückblieben. Zudem lernen sie häufig an Gesamtschulen, wo Bildungsaufstiege leichter möglich sind. Allerdings bieten auch Gesamtschulen einen zum Hauptschulabschluss führenden Bildungsgang an (analog finden sich in einigen Bundesländern Schulen mit mehreren Bildungsgängen). Die Zuordnung der Gesamtschulen zu den Realschulen und Gymnasien kann daher kritisch gesehen werden, zumal Gesamtschulen nicht in allen Regionen Deutschlands verbreitet sind. Ergänzend wird deshalb die „herkömmliche“ auf das dreigliedrige Schulsystem bezugnehmende Operationalisierung des Erfolgsindikators (Haupt-/Förderschule vs. Realschule/Gymnasium) verwendet. Die Schülerschaft an Gesamtschulen ( $\mathrm{N}=376$ ), für die datenbedingt keine Information zum konkret besuchten Bildungsgang vorliegt, wird in der ergänzenden Analyse ausgeschlossen. Es sei vorweggenommen, dass ein Ergebnisvergleich zwischen den Modellen mit den zwei verschieden konstruierten Erfolgsindikatoren kaum Unterschiede offenlegt.

${ }^{63}$ Frühgeburten sind in Deutschland relativ seltene Ereignisse (ca. $10 \%$ der Lebendgeburten). Wegen geringer Zellbesetzungen wird ein weites Kriterium verwendet, und zwar die Geburt vor Vollendung der 39. SSW.

${ }^{64}$ Eltern wurden gefragt: „Sind bei Ihrem Kind in den ersten 4 Lebenswochen nach der Geburt Probleme aufgetreten?" Wenn ja, konnten Eltern mehrere Antwortmöglichkeiten ankreuzen: Schwierigkeiten bei der Atmung/Anpassungsstörungen, Infektion, Gelbsucht, Untergewicht/Frühgeburt, Sonstige, Verlegung in Kinderklinik. Die Variable nimmt den Wert 1 an, wenn Eltern die Frage mit „Ja“ beantworteten, ansonsten 0.

${ }^{65}$ Die Schichtzugehörigkeit setzt sich gemäß dem sozialepidemiologischen Standard aus den Merkmalen Bildung/Ausbildung, berufliche Stellung und Einkommen zusammen. Den Statusmerkmalen werden Punktwerte zugeschrieben und diese zu einem Index aufaddiert (Lampert und Kroll 2006: 311).
} 
Sprache, werden erfasst über die Familiensprache (Deutsch, Deutsch und andere Sprache, kein Deutsch) und Zuzugszeitpunkt (Zuwanderung vor/nach der Geburt des Kindes).

Die Messung der Einbindung in frühe Bildungskontexte erfolgt über den Besuch einer Kindertagesstätte (kein Besuch, Eintritt mit zwei Jahren oder früher, Eintritt mit drei Jahren oder später) sowie die Präventionsneigung (Teilnahme an den U-Untersuchungen für Kinder; metrische Skala: 0, ..., 9 Untersuchungen).

Kontrollvariablen sind Geschlecht und Alter (in Monaten) des Kindes, die Stabilität der Familiensituation (Zwei-Elternfamilie vs. Ein-Elternfamilie), Alter der Mutter bei Geburt, Parität (Anzahl geborener Kinder der Mutter), pränataldiagnostische Risiken (mindestens ein Befund ${ }^{66}$, Alkoholkonsum und Gewichtszunahme (Proxy für Mangelernährung: weniger als $12 \mathrm{~kg}$ ) während der Schwangerschaft und Region (Ost/West; Stadt/Land). Für fehlende Angaben wird in den Analysen kontrolliert.

Im Ergebnisteil wird nun zunächst ein deskriptiver Variablenüberblick gegeben (7.4.1). In den multivariaten Analysen werden dann Ungleichheiten bezüglich der lebensanfänglichen (Geburtsergebnis) und schulischen Ausgangslage (Schulfähigkeit) untersucht (7.4.2). Schließlich ist zu prüfen, ob es über die gemeinsame Berücksichtigung aller Modellgrößen (Schwangerschaftseinflüsse, Geburtsergebnis, Elternhaus, Schulfähigkeit) gelingt, Noten- und bildungspositionelle Nachteile aufseiten der Migrantennachkommen aufzuklären (7.4.3).

\subsection{Ergebnisse}

\subsubsection{Deskriptive Statistiken zum Schulerfolg und den zentralen Modellvariablen}

Wie Tabelle 7.1 zeigt, fallen Noten- und Positionsdifferenzen je nach ethnischer Gruppe unterschiedlich aus. In der Primarstufe erzielen türkischstämmige Kinder die niedrigsten Noten während Spätaussiedlerkinder vergleichsweise besser abschneiden. Das gilt für das Unterrichtsfach Deutsch wie für Mathematik, wobei die Differenzen für die Deutschnote größer sind. Auch haben Migrantenkinder häufiger Lern- und Verhaltensprobleme im Schulalltag. Türkischstämmige Kinder werden zudem häufiger vom Schulbesuch (11\%) zurückgestellt. Im Sekundarbereich bietet sich ein ähnliches Muster. Türkischstämmige Kinder- und Jugendliche besuchen fast zu gleichen Teilen entweder einen weiterführenden oder niedrigen Schultyp. Im Gegensatz beträgt der Positionsabstand zwischen deutscher Schülerschaft (18\%) und Spätaussiedlernachkommen (28\%) nur 10 Prozentpunkte.

Tabelle 7.2 zeigt, ob bedeutsame Relationen zwischen der ethnischen Zugehörigkeit und den Modellvariablen vorliegen. Je nach Herkunftsgruppe ist dies empirisch mehr oder weniger der Fall: Die Prävalenzen für zu frühe Geburten, niedriges Geburtsgewicht und postnatale Gesundheitsprobleme sind bei türkischstämmigen Kindern am höchsten. Auch rauchen türkischstämmige Frauen gegenüber Deutschen und Spätaussiedlerinnen öfter während der

\footnotetext{
${ }^{66}$ Sobald mindestens eine Indikation (Schwangerschaftsdiabetes, Bluthochdruck, Infektion, Sonstige) vorlag, nimmt die Variable den Wert 1 an, ansonsten 0. Zudem wird dafür kontrolliert, dass Frauen nicht wissen, ob ein Risiko bestand, was auf eine unregelmäßige Nutzung der Schwangerenvorsorge hindeutet.
} 
Schwangerschaft. Neugeborene aus Spätaussiedlerfamilien kommen zwar auch verfrüht zur Welt, sind aber direkt nach der Geburt tendenziell schwerer als Deutsche und haben in signifikant geringerem Umfang lebensanfängliche Gesundheitsprobleme. Es zeigen sich auch ausgeprägte soziale und kulturelle Unterschiede: Spätaussiedlerkinder finden, bemessen an der familiären Sozialschichtzugehörigkeit, vergleichsweise günstige, türkischstämmige Kinder hingegen weniger lernvorteilhafte Bedingungen vor. Ungleich ist besonders der Wohnraum verteilt. Relativ beengt leben vor allem türkische Familien.

Tabelle 7.1: Bildungsmerkmale nach ethnischer Herkunft (getrennt für Primar-/Sekundarstufe)

\begin{tabular}{|c|c|c|c|c|}
\hline & Deutsch & Türkisch & Spätaussiedler & Sonstige \\
\hline GrundschülerInnen (Klassen 3-4) & $\mathrm{N}=1.632$ & $\mathrm{~N}=86$ & $\mathrm{~N}=95$ & $\mathrm{~N}=201$ \\
\hline Deutschnote $^{12}$ & $2,3(0,8)$ & $3,0(0,9)$ & $2,6(0,9)$ & $2,5(0,8)$ \\
\hline Mathenote $^{12}$ & $2,3(0,9)$ & $2,8(0,9)$ & $2,5(0,8)$ & $2,3(0,9)$ \\
\hline Zurückstellung vom Schulbesuch ${ }^{1}$ & $5,2 \%$ & $11,6 \%$ & $6,3 \%$ & $5,5 \%$ \\
\hline \multicolumn{5}{|l|}{ Sozialverhalten $^{1}$} \\
\hline Unauffällig & $72,6 \%$ & $52,3 \%$ & $68,4 \%$ & $68,7 \%$ \\
\hline Grenzwertig & $14,5 \%$ & $19,8 \%$ & $17,9 \%$ & $16,4 \%$ \\
\hline Auffällig & $12,9 \%$ & $27,9 \%$ & $13,7 \%$ & $14,9 \%$ \\
\hline Bewältigung des Schulalltags ${ }^{12}$ & $80,6(14,5)$ & $68,6(13,9)$ & $75,1(14,2)$ & $76,6(14,1)$ \\
\hline Klassenstufe & $3,9(0,8)$ & $4,0(0,9)$ & $4,0(0,8)$ & $3,9(0,9)$ \\
\hline \multicolumn{5}{|l|}{ Kontrollvariablen } \\
\hline Alter des Kindes (in Monaten) ${ }^{1}$ & $113,8(10,2)$ & $117,2(11,1)$ & $115,2(11,8)$ & $114,0(12,2)$ \\
\hline Mädchen & $48,1 \%$ & $38,4 \%$ & $50,5 \%$ & $53,2 \%$ \\
\hline Westdeutschland $^{12}$ & $58,0 \%$ & $83,7 \%$ & $81,1 \%$ & $76,1 \%$ \\
\hline \multicolumn{5}{|l|}{ Gemeindegröße $\mathrm{e}^{12}$} \\
\hline Ländlich/Kleinstädtisch & $56,8 \%$ & $26,7 \%$ & $33,7 \%$ & $27,9 \%$ \\
\hline Mittelstädtisch & $26,5 \%$ & $30,2 \%$ & $32,6 \%$ & $36,3 \%$ \\
\hline Großstädtisch & $16,7 \%$ & $43,0 \%$ & $33,7 \%$ & $35,8 \%$ \\
\hline SekundarschülerInnen (Klassen 5-9) & $\mathrm{N}=3.171$ & $\mathrm{~N}=118$ & $\mathrm{~N}=198$ & $\mathrm{~N}=363$ \\
\hline \multicolumn{5}{|l|}{ Besuchte Schulform $^{12}$} \\
\hline Haupt-/Förderschule & $18,3 \%$ & $48,3 \%$ & $28,8 \%$ & $27,3 \%$ \\
\hline Weiterführende Schulform & $81,7 \%$ & $51,7 \%$ & $71,2 \%$ & $72,7 \%$ \\
\hline Zurückstellung vom Schulbesuch & $7,4 \%$ & $9,3 \%$ & $6,1 \%$ & $6,9 \%$ \\
\hline \multicolumn{5}{|l|}{ Sozialverhalten $^{2}$} \\
\hline Unauffällig & $71,9 \%$ & $68,6 \%$ & $57,6 \%$ & $62,0 \%$ \\
\hline Grenzwertig & $14,9 \%$ & $11,0 \%$ & $21,2 \%$ & $17,3 \%$ \\
\hline Auffällig & $13,2 \%$ & $20,4 \%$ & $21,2 \%$ & $20,7 \%$ \\
\hline Bewältigung des Schulalltags ${ }^{12}$ & $73,5(15,0)$ & $63,8(13,3)$ & $70,4(13,5)$ & $70,2(14,7)$ \\
\hline Klassenstufe $^{2}$ & $7,2(1,3)$ & $7,2(1,3)$ & $7,1(1,3)$ & $7,1(1,3)$ \\
\hline \multicolumn{5}{|l|}{ Kontrollvariablen } \\
\hline Alter des Kindes (in Monaten) ${ }^{1}$ & $156,6(17,2)$ & $159,8(16,8)$ & $156,6(18,2)$ & $156,1(17,8)$ \\
\hline Mädchen & $47,8 \%$ & $43,2 \%$ & $53,5 \%$ & $46,6 \%$ \\
\hline Westdeutschland $^{12}$ & $64,9 \%$ & $90,7 \%$ & $93,4 \%$ & $85,6 \%$ \\
\hline \multicolumn{5}{|l|}{ Gemeindegröße $\mathrm{e}^{12}$} \\
\hline Ländlich/Kleinstädtisch & $52,3 \%$ & $26,3 \%$ & $31,8 \%$ & $30,6 \%$ \\
\hline Mittelstädtisch & $28,3 \%$ & $29,6 \%$ & $41,4 \%$ & $30,6 \%$ \\
\hline Großstädtisch & $19,4 \%$ & $44,1 \%$ & $26,8 \%$ & $38,8 \%$ \\
\hline
\end{tabular}

Anmerkungen: Prozent-/Mittelwerte, Standardabweichung in Klammern; Signifikanz: ${ }^{1}$ Kontrast: Dt.-Türk. ( $\mathrm{p}<0.05),{ }^{2}$ Kontrast: Dt.-Spät ( $<$ 0.05); Eigene Analysen auf Basis des KiGGS (2003-2006). 
Tabelle 7.2: Säuglingsgesundheit, Schwangerschafts- und Familienmerkmale nach ethnischer Herkunft ${ }^{\text {a }}$

\begin{tabular}{|c|c|c|c|c|}
\hline & $\begin{array}{c}\text { Deutsch } \\
\mathrm{N}=4.803\end{array}$ & $\begin{array}{l}\text { Türkisch } \\
\mathrm{N}=204\end{array}$ & $\begin{array}{c}\text { Spätaussiedler } \\
\mathrm{N}=293 \\
\end{array}$ & $\begin{array}{l}\text { Sonstige } \\
\mathrm{N}=564\end{array}$ \\
\hline \multicolumn{5}{|l|}{ Säuglingsgesundheit und Schwangerschaft } \\
\hline \multicolumn{5}{|l|}{ Neugeborenengesundheit } \\
\hline $\begin{array}{l}\text { Geburtsgewicht (in kg) }{ }^{1} \\
\text { (Anzahl fehlender Werte) }\end{array}$ & $\begin{array}{l}3381,1(538,9) \\
(79)\end{array}$ & $\begin{array}{c}3314,0(559,0) \\
(13)\end{array}$ & $\begin{array}{c}3402,5(510,4) \\
\text { (4) }\end{array}$ & $\begin{array}{c}3366,1(519,2) \\
\text { (19) }\end{array}$ \\
\hline \multicolumn{5}{|l|}{ Schwangerschaftsdauer ${ }^{12}$} \\
\hline $\begin{array}{l}\text { Frühgeborene }(<37 \mathrm{SSW}) \\
\text { Frühe Termingeborene }(37-38 \mathrm{SSW}) \\
\quad(\text { Anzahl fehlender Werte) }\end{array}$ & $\begin{array}{c}5,6 \% \\
15,4 \% \\
(490)\end{array}$ & $\begin{array}{c}8,6 \% \\
25,2 \% \\
(54)\end{array}$ & $\begin{array}{c}7,3 \% \\
19,3 \% \\
(75)\end{array}$ & $\begin{array}{r}11,3 \% \\
14,7 \% \\
(122)\end{array}$ \\
\hline Gesundheitsprobleme nach Geburt $^{2}$ & $25,8 \%$ & $28,9 \%$ & $18,8 \%$ & $23,9 \%$ \\
\hline \multicolumn{5}{|l|}{ Lebensführung während Schwangerschaft } \\
\hline $\begin{array}{l}\text { Tabakkonsum }^{12} \\
\text { Nein } \\
\text { Ab und zu } \\
\text { Regelmäßig }\end{array}$ & $\begin{array}{c}84,0 \% \\
12,3 \% \\
3,7 \%\end{array}$ & $\begin{array}{c}74,0 \% \\
20,6 \% \\
5,4 \%\end{array}$ & $\begin{array}{c}91,1 \% \\
5,5 \% \\
{[2,4] \%}\end{array}$ & $\begin{array}{l}79,1 \% \\
14,7 \% \\
6,2 \%\end{array}$ \\
\hline Alkoholkonsum $^{12}$ & $14,8 \%$ & {$[2,0] \%$} & $9,9 \%$ & $12,9 \%$ \\
\hline $\begin{array}{l}\text { Gewichtszunahme }<12 \mathrm{~kg} \\
\quad(\text { Anzahl fehlender Werte) }\end{array}$ & $\begin{array}{c}50,1 \% \\
(282)\end{array}$ & $\begin{array}{c}54,2 \% \\
(14)\end{array}$ & $\begin{array}{l}53,2 \% \\
(29)\end{array}$ & $\begin{array}{c}51,5 \% \\
(40)\end{array}$ \\
\hline \multicolumn{5}{|l|}{ Schwangerschaftsrisiken } \\
\hline $\begin{array}{l}\text { Mindestens ein Risikobefund } \\
\text { Weiß nicht } \\
\text { (Anzahl fehlender Werte) } \\
\text { Größe der Mutter }^{12}\end{array}$ & $\begin{array}{c}14,0 \% \\
5,1 \% \\
(283) \\
166,9(6,0)\end{array}$ & $\begin{array}{c}8,4 \% \\
30,5 \% \\
\quad(14) \\
161,7(5,6)\end{array}$ & $\begin{array}{c}9,5 \% \\
22,7 \% \\
(29) \\
163,9(5,7)\end{array}$ & $\begin{array}{c}11,6 \% \\
17,6 \% \\
(40) \\
164,8(6,7)\end{array}$ \\
\hline \multicolumn{5}{|l|}{ Familiäre Herkunft } \\
\hline $\begin{array}{l}\text { Soziale Schicht }(\text { Winkler-Index) } \\
\text { Niedrig } \\
\text { Mittel } \\
\text { Hoch }\end{array}$ & $\begin{array}{l}22,2 \% \\
51,2 \% \\
26,7 \%\end{array}$ & $\begin{array}{l}71,1 \% \\
26,5 \% \\
{[2,5] \%}\end{array}$ & $\begin{array}{l}43,3 \% \\
44,7 \% \\
12,0 \%\end{array}$ & $\begin{array}{l}28,9 \% \\
44,0 \% \\
27,1 \%\end{array}$ \\
\hline Wohnraumfläche (pro Person) $)^{12}$ & $31,4(11,6)$ & $21,6(8,8)$ & $26,9(9,7)$ & $28,5(12,4)$ \\
\hline Ein-Eltern-Familie & $12,6 \%$ & $14,2 \%$ & $9,6 \%$ & $13,8 \%$ \\
\hline $\begin{array}{l}\text { Familiensprache }{ }^{12} \\
\text { Deutsch } \\
\text { Deutsch und andere Sprache } \\
\text { Kein Deutsch }\end{array}$ & $\begin{array}{c}99,1 \% \\
0,9 \% \\
0 \%\end{array}$ & $\begin{array}{l}11,8 \% \\
75,5 \% \\
12,8 \%\end{array}$ & $\begin{array}{c}39,6 \% \\
54,6 \% \\
5,8 \%\end{array}$ & $\begin{array}{l}41,0 \% \\
44,7 \% \\
14,3 \%\end{array}$ \\
\hline Zuwanderung nach Geburt ${ }^{12}$ & $0 \%$ & $8,3 \%$ & $37,9 \%$ & $20,9 \%$ \\
\hline Alter der Mutter zur Geburt ${ }^{12}$ & $28,2(4,7)$ & $24,6(4,7)$ & $26,5(5,2)$ & $28,2(5,2)$ \\
\hline Parität & $1,79(0,95)$ & $1,81(1,00)$ & $1,80(1,01)$ & $1,85(1,09)$ \\
\hline \multicolumn{5}{|l|}{ Entwicklungsinvestitionen } \\
\hline $\begin{array}{l}\text { Kindergartenbesuch (Eintrittsalter) }{ }^{12} \\
\text { Kein KiTa-Besuch } \\
0-35 \text { Monate } \\
36 \text { Monate und älter } \\
\text { (Anzahl fehlender Werte) }\end{array}$ & $\begin{array}{c}11,9 \% \\
31,0 \% \\
57,1 \% \\
(76)\end{array}$ & $\begin{array}{c}25,0 \% \\
8,5 \% \\
66,5 \% \\
(16)\end{array}$ & $\begin{array}{c}18,6 \% \\
12,1 \% \\
69,3 \% \\
(19)\end{array}$ & $\begin{array}{l}16,3 \% \\
19,9 \% \\
63,8 \% \\
(31)\end{array}$ \\
\hline Präventionsstatus (U-Teilnahme) ${ }^{12}$ & $8,4(1,7)$ & $7,5(2,6)$ & $6,6(3,4)$ & $7,0(3,2)$ \\
\hline
\end{tabular}

Anmerkungen: Prozent-/Mittelwerte, Standardabweichung in runden Klammern, Werte in eckigen Klammern basieren auf Fallzahlen $<10,{ }^{a}$ Gesamtstichprobe $(\mathrm{N}=5.864)$, Signifikanz: ${ }^{1}$ Dt.-Türk. $(\mathrm{p}<0.05),{ }^{2}$ Dt.-Spät ( $<<0.05)$; Eigene Analysen auf Basis des KiGGS (2003-2006). 
In sprachkultureller Hinsicht wachsen (besonders türkischstämmige) Migrantenkinder seltener mit Deutsch als Familiensprache auf. Auch in Kindertagesstätten sind Kinder mit Migrationshintergrund seltener und erst in einem höheren Lebensalter verstärkt präsent. Beim Vorsorgestatus haben besonders Kinder aus Spätaussiedlerfamilien - selbst wenn der Zuwanderungszeitpunkt in Rechnung gestellt wird (Ergebnis nicht dargestellt) - Lücken: Von insgesamt neun Früherkennungsuntersuchungen werden im Mittel nur 6,6 genutzt.

\subsubsection{Ethnische Disparitäten in der gesundheitlichen und schulischen Ausgangslage}

Tabelle 7.3 stellt die Ergebnisse multipler Regressionsanalysen zur Frage bereit, ob zwischen den ethnischen Gruppen bedeutsame Gesundheitsdifferenzen zum Lebensbeginn vorliegen.

Je nach Skalierung des Kriteriums werden entweder logistische (Termingeburt, postnatale Gesundheit) oder lineare Regressionen (Geburtsgewicht) geschätzt. Insgesamt werden jeweils drei Regressionsmodelle spezifiziert. Die Aussagen zum Modellvergleich logistischer Regressionsbefunde basieren auf average marginal effects (Mood 2010; Winship und Mare 1984).

In die Ausgangsmodelle gehen zunächst nur die ethnische Herkunftsgruppe (und die Kontrollvariablen Alter und Geschlecht des Kindes) ein (M1abc). Danach werden die Indikatoren zu Lebensführung und Ressourcen aufgenommen (M2abc), um zu prüfen, ob Startdifferenzen nach deren Kontrolle abnehmen. In einem dritten Schritt werden medizinische Einflussfaktoren im Regressionsmodell berücksichtigt (M3abc).

Wie die Ausgangsmodelle zeigen (M1abc), steigt, im Vergleich zur Referenzgruppe, für türkische Kinder die Wahrscheinlichkeit im Mittel um 11 Prozentpunkte, zu früh ins Leben zu starten. Auch sind sie im Mittel 77 Gramm leichter und die Wahrscheinlichkeit keine postnatalen gesundheitliche Probleme zu haben, sinkt tendenziell um 4 Prozentpunkte $(p<0,20)$. Auch Kinder aus Spätaussiedlerfamilien haben eine höhere Wahrscheinlichkeit zu früh geboren zu werden, mit knapp 6 Prozentpunkten ist diese aber nur etwa halb so groß. Zudem haben diese Kinder im Mittel ein höheres Geburtsgewicht (der Unterschied von 23 g zur Referenzgruppe ist allerdings nicht signifikant) und weniger Gesundheitsprobleme nach der Geburt. Diese Geburtsdifferenzen stützen Hypothese 1, die insbesondere für türkischstämmige Säuglinge lebensanfängliche Nachteile konstatiert.

Wie die Schätzergebnisse des zweiten Modellblocks ( $M 2 c$ ausgenommen) zeigen, lassen sich diese Unterschiede über familiäre und verhaltensbezogene Merkmale aufklären. Nach Berücksichtigung des Risikohandelns und sozialer Ressourcen (M2ab) schwächen sich die Differenzen zwischen türkischen und deutschen Kindern ab und verlieren an Signifikanz. Residualeffekte für das Geburtsgewicht $(M 2 b)$ lassen sich für die türkische Gruppe, wie $M 3 b$ zeigt, über sozialmedizinische Faktoren restlos aufklären. ${ }^{67}$ Spätaussiedlerkindern wiegen nun sogar im Mittel 53 Gramm mehr als einheimische Kinder $(\mathrm{p}<0,17)$.

\footnotetext{
${ }^{67}$ Der Mediationsbeitrag medizinischer Faktoren für ethnische Geburtsgewichtsdifferenz ist nahezu allein der Konstitution (Größe) zuzurechnen. Entfernt man die Größenvariable aus M3b und berücksichtigt nur Alter, Parität und Komplikationen, dann fallen die gewichtsbezogenen Residualeffekte für die ethnischen Gruppen (Türkischstämmige: $b=-56,149$; Spätaussiedler: $b=-23,327$ ) ähnlich hoch aus wie in M2b.
} 


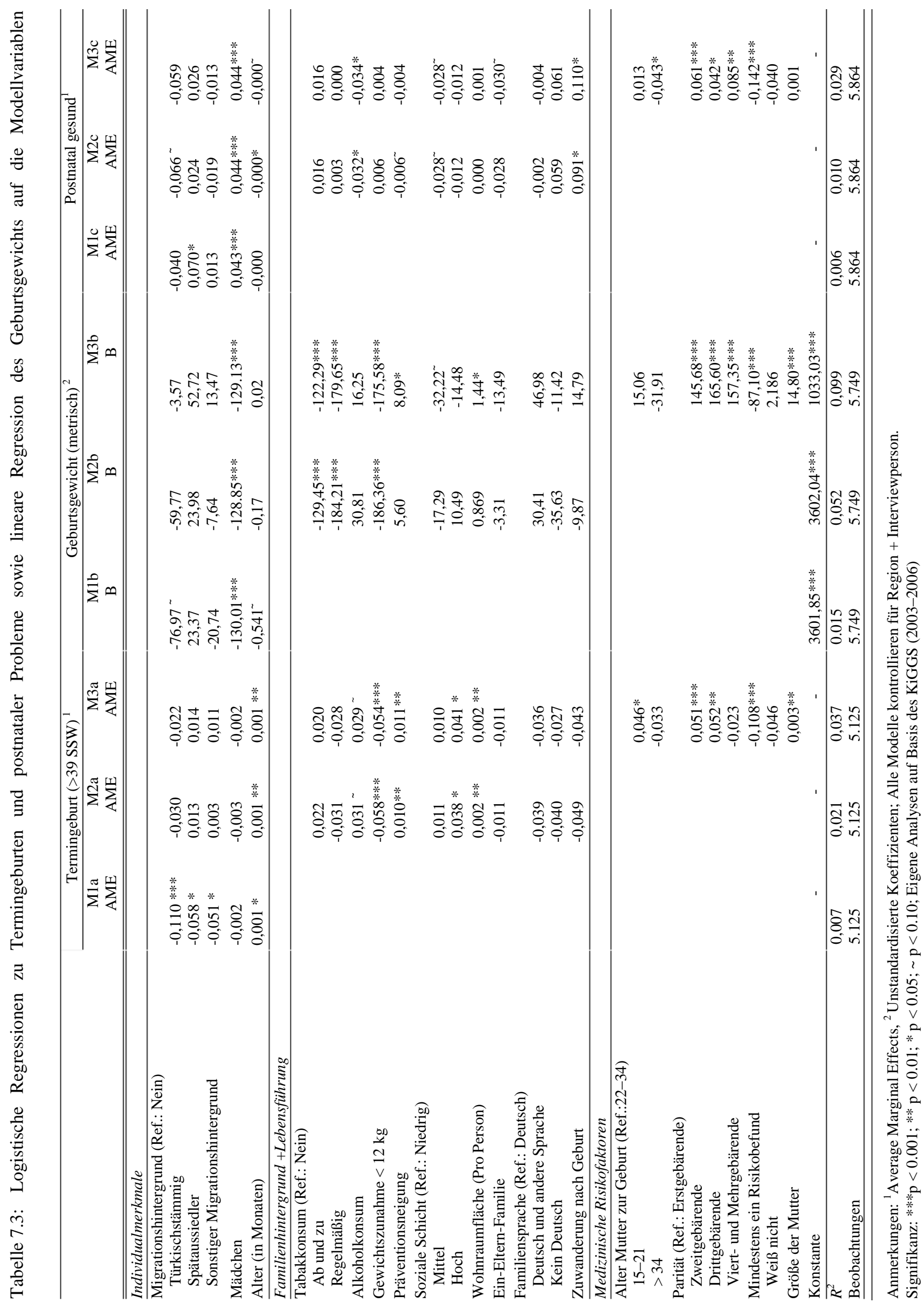


Insgesamt besitzen soziale und verhaltensbezogene Merkmale gesundheitliche Relevanz. Allerdings variieren die Effektstärken, je nach Gesundheitskriterium, zum Teil erheblich. Besonders stabil ist der Regressionsschätzer für das Proxy zum Präventivhandeln während der Schwangerschaft: Je höher die Präventionsneigung, desto unwahrscheinlicher sind Geburten vor dem errechneten Termin und niedriges Geburtsgewicht. ${ }^{68}$

Tabelle 7.4 zeigt die Ergebnisse multipler logistischer Regressionen mit Blick auf die Frage, ob sich lebensanfängliche gesundheitliche Ungleichheit zum Zeitpunkt der Einschulung in höheren Raten der Rückstellung vom Schulbesuch niederschlägt und einen eigenständigen Beitrag zum Verständnis früher Bildungsungleichheit leistet. ${ }^{69} \mathrm{Zu}$ diesem Zweck werden mehrere Regressionsmodelle spezifiziert. In das Ausgangsmodell gehen die ethnischen Gruppen und Kontrollvariablen ein: Im Vergleich zu Kindern ohne ausländische Wurzeln, werden Türkischstämmige, nicht aber Spätaussiedlerkinder, eher vom Grundschulbesuch zurückgestellt (M4). Nach Aufnahme der Lebensführung während der Schwangerschaft, dem Frühgeborenenstatus und der Säuglingsgesundheit schwächen sich, wie M5 zeigt, die Einschulungsunterschiede zwar ab, verlieren aber nur leicht an Signifikanz. Im Einklang mit Hypothese 6 haben Frühgeborene und intrauterin regelmäßig Tabakrauch ausgesetzte Kinder im Mittel eine höhere Rückstellungswahrscheinlichkeit. Kinder mit postnatalen Gesundheitsproblemen haben auch eine etwas höhere Rückstellungswahrscheinlichkeit. Bedeutsam an der Einschulungsschwelle ist auch der Indikator zur Mangelernährung $(<12 \mathrm{~kg}$ Gewichtszunahme während der Schwangerschaft). Entgegen der theoretischen Erwartung statistisch nicht bedeutsam sind die U-Teilnahme und der Kindergartenbesuch.

Die Befunde unterstreichen die Relevanz vor- nicht aber nachgeburtlicher Investitionen für das Einschulungsergebnis. Wider Erwarten können jedoch die Entwicklungsnachteile der türkischstämmigen Gruppe weder über die Lebensführung während der Schwangerschaft noch über die formelle Kinderbetreuung mitaufgeklärt werden: Wie M6 belegt, bleibt der Schätzer der türkischen Gruppe in Höhe und Bedeutsamkeit nahezu unverändert.

Welchen Erklärungsbeitrag der soziokulturelle Argumentationsstrang leistet, wird zunächst getrennt von embryonalen Entwicklungsumständen in zwei weiteren Modellen untersucht. Wie der stark reduzierte Schätzer für türkischstämmige Kinder in M7 zeigt, vermitteln sozioökonomische Ressourcen Schuleingangsdifferenzen zwischen türkischstämmigen und deutschen Kindern fast vollständig. Von Familiensprache und Zuwanderungszeitpunkt geht kein bedeutsames Mediationspotential aus, wie M8 belegt. Auch die gemeinsame Berücksichti-

\footnotetext{
${ }^{68}$ Für die Neugeborenengesundheit zeigt sich ein umgekehrter Effekt: Je höher die Präventionsneigung, desto schlechter die Gesundheit. Dieser Befund ist womöglich einem Endogenitätsproblem geschuldet und eher so zu deuten, dass Eltern dann eine geringere Vorsorgebereitschaft haben, wenn Kinder gesund sind, d.h. der Präventionsbedarf wird bei Abwesenheit gesundheitlicher Probleme aus subjektiver Sicht eher unterschätzt.

${ }^{69}$ Aufgrund der hohen Korrelation zwischen Schwangerschaftsdauer und Geburtsgewicht und dem Ergebnis, dass sich die Herkunftsgruppen stärker hinsichtlich der Schwangerschaftsdauer unterscheiden, geht das Geburtsgewicht nicht in die Bildungsanalysen ein. Eine gleichzeitige Berücksichtigung führt nicht zu inhaltlich anderen Schlussfolgerungen.
} 
gung vorgeburtlicher und soziokultureller Faktoren schwächt die Differenzen nicht weiter ab (M9). Festzuhalten ist, dass Risikohandeln (Rauchen), Frühgeburtlichkeit und Gesundheitsstatus auch nach Ressourcenkontrolle weiterhin eine bedeutsame Rolle für die Schulfähigkeit spielen, was die eingangs postulierte These stützt, dass Startnachteile über die Vorschulzeit kumulieren.

Tabelle 7.4: Zurückstellung vom Schulbesuch (logistische Regression, Gesamtstichprobe)

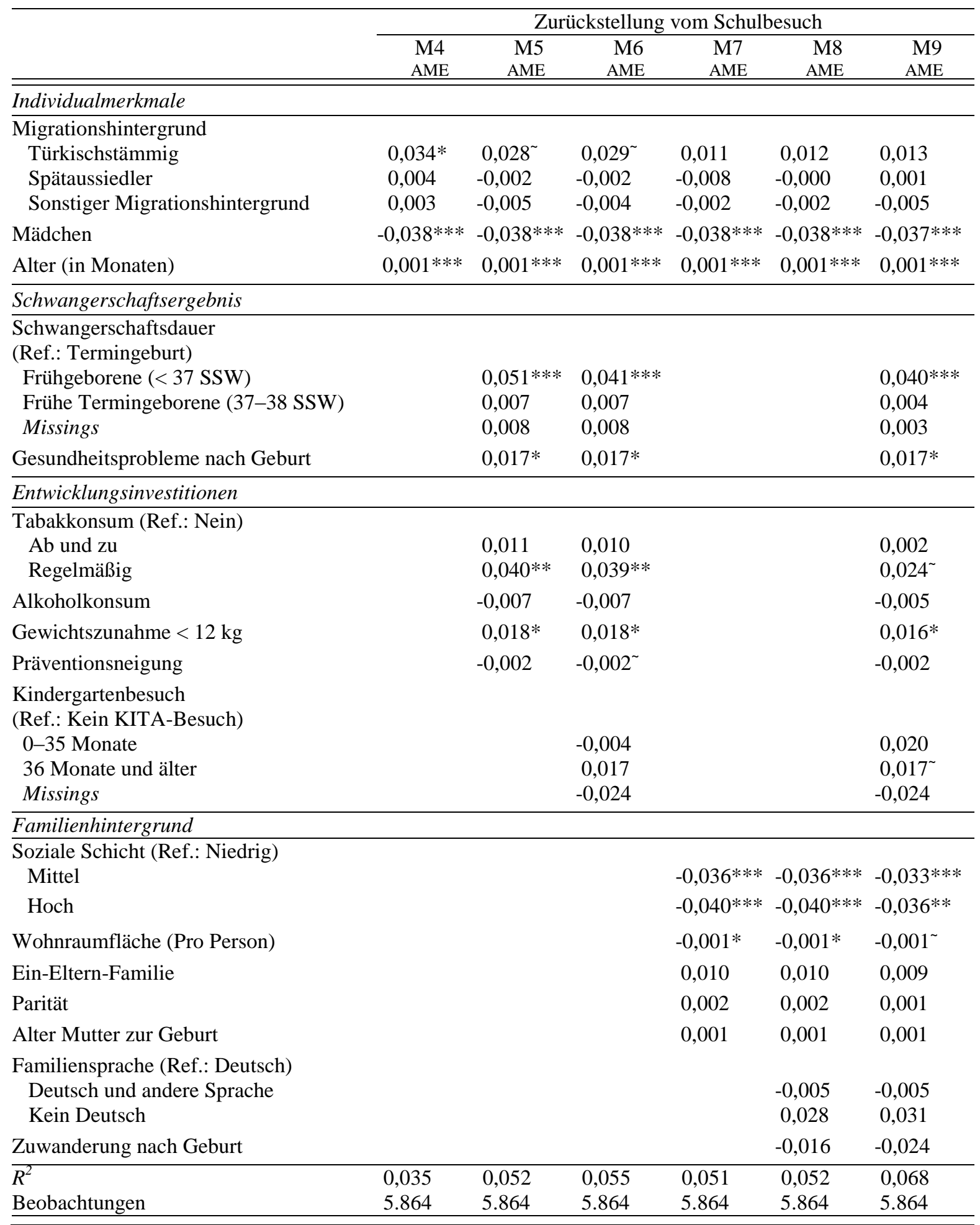

Anmerkungen: ${ }^{1}$ Average Marginal Effects; Alle Modelle kontrollieren für Region + Interviewperson.

Signifikanz: $* *$ p $<0.001 ; * * \mathrm{p}<0.01 ; * \mathrm{p}<0.05 ; \sim \mathrm{p}<0.10$; Eigene Analysen auf Basis des KiGGS (2003-2006). 


\subsubsection{Ethnische Disparitäten in den Grundschulnoten und im Sekundarschulbesuch}

Ob Notendifferenzen zwischen Migrantennachkommen und der deutschen Schülerschaft präund postnatalen leistungsrelevanten Bedingungen geschuldet sind, wird mit multiplen linearen Regressionen überprüft. Wie zuvor wird hierbei schrittweise vorgegangen. Gemäß der analytischen Trennung schwangerschaftsbezogener und vorschulischer Erfolgsrisiken gehen die Ursachenbündel sowohl separat als auch gemeinsam in die Schätzung ein.

Tabelle 7.5 enthält die Schätzer für die Deutschnote, wobei negative Vorzeichen schlechtere Noten anzeigen. Zwischen den ethnischen Gruppen besteht ein ausgeprägtes Notengefälle (M10). Wie M11 zeigt, ist die Frühgeburt nur tendenziell mit der Deutschnote assoziiert $(\mathrm{p}<0,15)$, Tabakkonsum aber umso eindeutiger: Kinder mit Müttern, die während der Schwangerschaft regelmäßig rauchten, schneiden fast eine halbe Note schlechter ab. Selbst unregelmäßiges Rauchen zieht Nachteile nach sich. Erwartungsgemäß sind eine stete Vorsorge von Geburt an und außerhäusliche Betreuung positiv leistungswirksam: Je kontinuierlicher Vorsorge genutzt wird, desto höher die Wahrscheinlichkeit für bessere Leistungsbewertungen (M11). Auch Kinder, die vor dem dritten Geburtstag außerhäuslich betreut wurden, profitieren in Form besserer Deutschnoten. Alkoholkonsum während der Schwangerschaft und postnatale Gesundheit sind hingegen notenirrelevant.

Für türkischstämmige Kinder reduziert sich unter Kontrolle der schwangerschafts- bzw. geburtsbezogenen und vorschulischen Faktoren die Ausgangsdifferenz von $b=-0,61$ (M10) auf $b=-0,51$ (M11), für Spätaussiedlerkinder von $b=-0,26$ auf $b=-0,20$. Ihr Beitrag zur Aufklärung ethnisch strukturierter Leistungen im Fach Deutsch ist somit gering. Auch ein verzögerter Schuleintritt, mit der Note erwartungsgemäß (Hypothese 5) negativ assoziiert, vermittelt die Erfolgsnachteile von Migrantenkindern nicht (M12).

Als Zwischenfazit lässt sich festhalten: Weder schwangerschaftsbezogene Faktoren noch Frühinvestitionen oder Schuleingangsunterschiede können den ethnischen Notengradienten erklären. Leisten stattdessen soziokulturelle Umstände einen Erklärungsbeitrag?

Im Einklang mit Hypothese 3 sind, wie M13 zeigt, niedrigere Noten Ausdruck ungleicher sozioökonomischer Lern-/Förderopportunitäten in der Familie: Für Spätaussiedlerkinder bestehen nach Kontrolle der sozialen Schichtzugehörigkeit und häuslichem Platzangebot nur geringe Residualeffekte fort. Nicht so für türkische Nachkommen, für die nach wie vor eine signifikante Notendifferenz zu verbuchen ist, und zwar auch nach Aufnahme (M14) sprachkultureller Gelegenheiten (Familiensprache, Zeitpunkt der Zuwanderung).

Ist ethnische Bildungsdifferenz durch prä- und postnatale Bedingungen verursacht, dann müsste deren gemeinsame regressionsanalytische Berücksichtigung, die in gesonderten Modellen fortbestehenden Nachteile für türkische Nachkommen aufklären. Wie M15 zeigt, ist die Notendifferenz zwischen Deutschen und Türkischstämmigen von 0,19-Punkten nun nicht mehr signifikant. Eine vollständige Mediation wird hierüber aber nicht erreicht. Vermitteln individuelle Eigenschaften die verbleibenden Herkunftseffekte? Wie gut Kinder den Schulalltag meistern und wie sie sich sozial verhalten, wird in M16 berücksichtigt. Wie in den Hypo- 
thesen 7 und 8 postuliert, sind beide Maße relevant: Je besser die Bewältigung des Schulalltags und je unauffälliger (bzw. konformer) das Sozialverhalten, desto höher der Notenertrag. Die Koeffizienten für die ethnischen Gruppen nähern sich dem Wert Null. Ferner schwächt sich auch der Misserfolg von Kindern, die während der Embryonalphase Rauchschadstoffen ausgesetzt waren (M15 vs. M16), ab, was als Indiz für die intrauterine Kompetenzprägung gewertet werden kann.

Für die Mathematiknote finden sich insgesamt ähnliche Befunde, obgleich die Dauer der Schwangerschaft weniger relevant ist (Tabelle A 7.1, Anhang). Als bedeutsame Prädiktoren erweisen sich prä- und postnatale Investitionen sowie die soziale Herkunft, über die sich ethnische differente Mathematiknoten vollständig aufklären lassen. Die Vorzeichen drehen sich nun für die Migrantengruppen sogar um.

Abschließend wird sich nun der Frage zugewandt, ob das theoretische Modell auch für die Aufklärung ethnischer Ungleichheit in der Sekundarschulbeteiligung empirisch greift. Methodisch wird in analogen Schritten vorgegangen. Statt linearer Regressionen werden entsprechend des binären Kriteriums logistische Regressionen gerechnet: Vorhergesagt wird der Besuch eines höheren (Mittelschule, Gesamtschule, Gymnasium) vs. niedrigen Schultyps (Haupt-/Förderschule). Tabelle 7.6 enthält die Befunde. Für den Modellvergleich werden durchschnittlich marginale Effekte berichtet. Wie M17 zeigt, ist der Besuch eines höheren Schultypus für türkische Nachkommen im Mittel um 21 Prozentpunkte, für Kinder aus Spätaussiedlerfamilien 7 Prozentpunkte wahrscheinlicher als für deutsche Kinder. Die Positionsdifferenzen nehmen, wie in Hypothese 2 (zum Einfluss der Schwangerschaft) und Hypothese 4 (zu Investitionen im Vorschulalter) angenommen, nach Berücksichtigung schwangerschaftsbezogener und vorschulischer Entwicklungsgrößen ab, und zwar um durchschnittlich 3 Prozentpunkte für beide Gruppen (M18). Für türkischstämmige Kinder bestehen aber im Gegensatz zu ethnisch-deutschen Nachkommen bedeutsame Differenzen fort. Dabei sind in M18 bis auf die postnatale Gesundheit und der Alkoholkonsum alle Variablen erwartungsgemäß mit dem Schulbesuch assoziiert: Frühgeborene $(\mathrm{p}=0,051)$ und intrauterin regelmäßig Tabakrauch ausgesetzte Kinder haben im Mittel eine geringere Wahrscheinlichkeit, statt die Haupt-/Förderschule eine höhere Schulform zu besuchen. Jene, die kontinuierlich am Krankheitsfrüherkennungsprogramm teilnahmen oder vor dem dritten Geburtstag formell betreut wurden, positionieren sich eher in höheren Schulformen. Umgekehrt ist dies, erwartungsgemäß (Hypothese 5), für vom Schulbesuch zurückgestellte Kinder um 15 Prozentpunkte unwahrscheinlicher; obgleich hiermit kein Erklärungsbeitrag für die Positionsdifferenzen verknüpft ist (M19). 
Tabelle 7.5: Lineare Regression der Deutschnote (GrundschülerInnen)

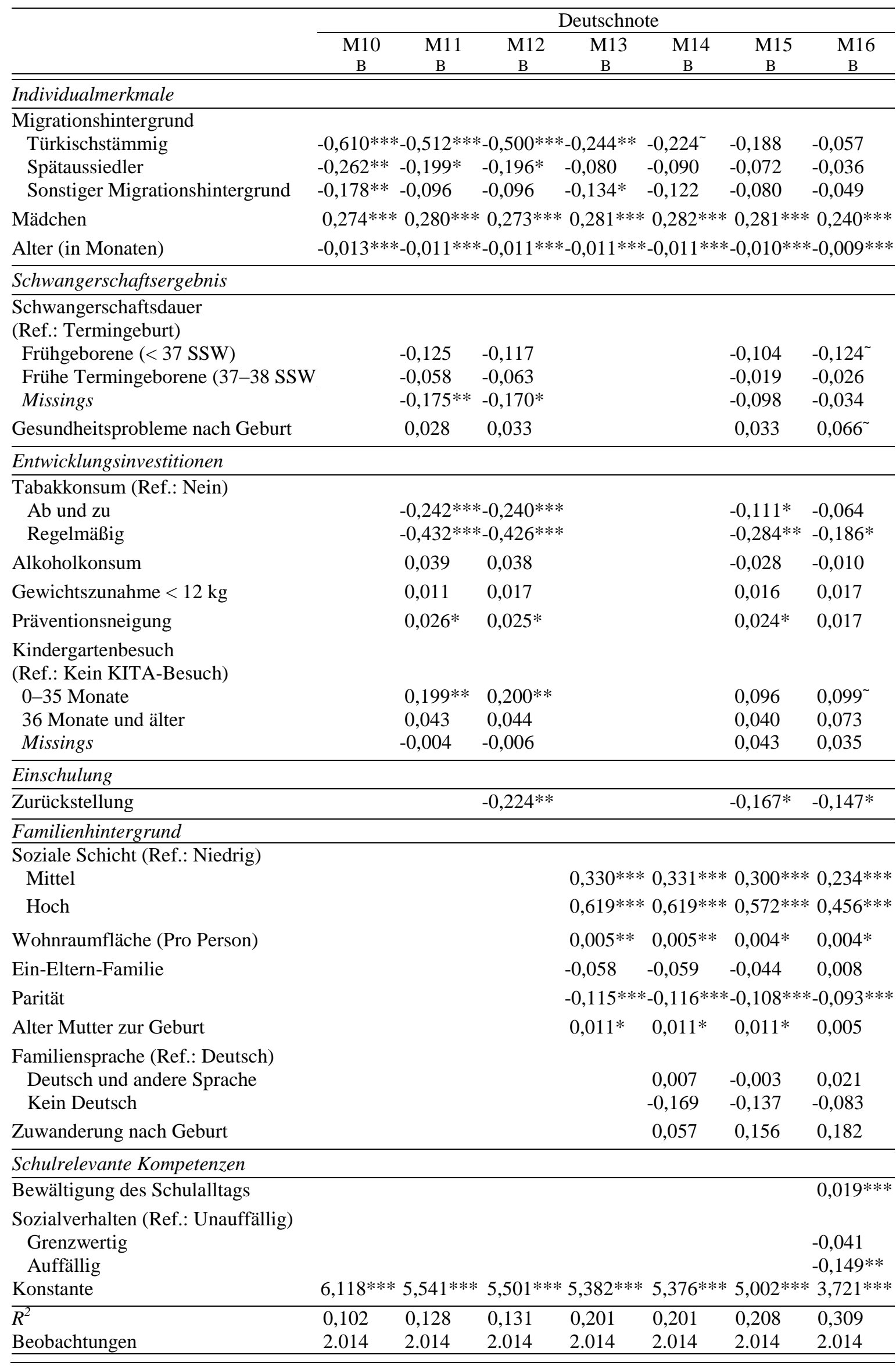

Anmerkungen: Unstandardisierte Koeffizienten; Alle Modelle kontrollieren für Region + Interviewperson.

Signifikanz: ${ }^{* *} \mathrm{p}<0.001 ; * * \mathrm{p}<0.01 ; * \mathrm{p}<0.05 ; \sim \mathrm{p}<0.10$; Eigene Analysen auf Basis des KiGGS (2003-2006). 
Tabelle 7.6: Logistische Regression des Schulbesuchs (SekundarschülerInnen, inkl. Gesamtschülerschaft)

\begin{tabular}{|c|c|c|c|c|c|c|c|}
\hline & \multicolumn{6}{|c|}{ Haupt-/Förderschulschulbesuch ${ }^{1}$} & \multirow{3}{*}{$\begin{array}{l}\text { M23 } \\
\text { AME }\end{array}$} \\
\hline & M17 & M18 & M19 & M20 & $\mathrm{M} 21$ & M22 & \\
\hline & AME & AME & AME & AME & AME & AME & \\
\hline \multicolumn{8}{|l|}{ Individualmerkmale } \\
\hline \multicolumn{8}{|l|}{ Migrationshintergrund } \\
\hline Türkischstämmig & $0,209 * * *$ & $0,171 * * *$ & $0,169 * * *$ & 0,047 & 0,032 & 0,033 & 0,028 \\
\hline Spätaussiedler & $0,075 * *$ & 0,041 & 0,043 & $-0,003$ & $-0,034$ & $-0,028$ & $-0,040$ \\
\hline Sonstiger Migrationshintergrund & $0,072 * * *$ & $0,035^{\sim}$ & $0,037^{\sim}$ & $0,033^{\sim}$ & 0,016 & 0,008 & 0,003 \\
\hline Mädchen & $-0,082 * * *$ & $-0,083 * * *$ & $-0,076^{* * *}$ & $-0,082 * * *$ & $-0,082 * * *$ & $-0,076 * * *$ & $-0,068 * * *$ \\
\hline Alter (in Monaten) & $0,002 * * *$ & $0,001 * * *$ & $0,001 * *$ & $0,001 * *$ & $0,001 * * *$ & $0,001 * *$ & $0,001 *$ \\
\hline \multicolumn{8}{|l|}{ Schwangerschaftsergebnis } \\
\hline \multicolumn{8}{|l|}{$\begin{array}{l}\text { Schwangerschaftsdauer } \\
\text { (Ref.: Termingeburt) }\end{array}$} \\
\hline Frühgeborene $(<37$ SSW $)$ & & $0,052^{\sim}$ & 0,041 & & & $0,049^{\sim}$ & $0,053 *$ \\
\hline Frühe Termingeborene (37-38 SSW) & & 0,020 & 0,016 & & & 0,005 & 0,000 \\
\hline Missings & & $0,047 *$ & $0,046^{*}$ & & & 0,023 & 0,022 \\
\hline Gesundheitsprobleme nach Geburt & & $-0,014$ & $-0,017$ & & & $-0,004$ & $-0,007$ \\
\hline \multicolumn{8}{|l|}{ Entwicklungsinvestitionen } \\
\hline \multicolumn{8}{|l|}{ Tabakkonsum (Ref.: Nein) } \\
\hline $\mathrm{Ab}$ und $\mathrm{zu}$ & & $0,120 * * *$ & $0,118 * * *$ & & & $0,066 * * *$ & $0,056 * * *$ \\
\hline Regelmäßig & & $0,218 * * *$ & $0,210 * * *$ & & & $0,115 * * *$ & $0,101 * * *$ \\
\hline Alkoholkonsum & & $-0,042 *$ & $-0,039 *$ & & & $-0,016$ & $-0,018$ \\
\hline Gewichtszunahme $<12 \mathrm{~kg}$ & & $0,026^{*}$ & $0,024^{\sim}$ & & & 0,017 & 0,018 \\
\hline Präventionsneigung & & $-0,012 * * *$ & $-0,012 * * *$ & & & $-0,008 * *$ & $-0,007 * *$ \\
\hline \multicolumn{8}{|l|}{$\begin{array}{l}\text { Kindergartenbesuch } \\
\text { (Ref.: Kein KITA-Besuch) }\end{array}$} \\
\hline 0-35 Monate & & $-0,073 * *$ & $-0,071 * *$ & & & $-0,021$ & $-0,025$ \\
\hline 36 Monate und älter & & $-0,020$ & $-0,024$ & & & $-0,015$ & $-0,018$ \\
\hline Missings & & 0,014 & 0,019 & & & 0,018 & 0,009 \\
\hline \multicolumn{8}{|l|}{ Einschulung } \\
\hline Zurückstellung & & & $0,150 * * *$ & & & $0,122 * * *$ & $0,119 * * *$ \\
\hline \multicolumn{8}{|l|}{ Familienhintergrund } \\
\hline \multicolumn{8}{|l|}{ Soziale Schicht (Ref.: Niedrig) } \\
\hline Mittel & & & & $-0,123 * * *$ & $-0,123 * * *$ & $-0,102 * * *$ & $-0,096 * * *$ \\
\hline Hoch & & & & $-0,320 * * *$ & $-0,321 * * *$ & $-0,288 * * *$ & $-0,278 * * *$ \\
\hline Wohnraumfläche (pro Person) & & & & $-0,002 * *$ & $-0,002 * *$ & $-0,002 * *$ & $-0,002 * *$ \\
\hline Ein-Eltern-Familie & & & & 0,014 & 0,015 & 0,008 & 0,008 \\
\hline Parität & & & & $0,052 * * *$ & $0,051 * * *$ & $0,047 * * *$ & $0,045 * * *$ \\
\hline Alter Mutter zur Geburt & & & & $-0,008 * * *$ & $-0,008 * * *$ & $-0,007 * * *$ & $-0,006 * * *$ \\
\hline \multicolumn{8}{|l|}{ Familiensprache (Ref.: Deutsch) } \\
\hline Deutsch und andere Sprache & & & & & 0,017 & 0,015 & 0,021 \\
\hline Kein Deutsch & & & & & $-0,014$ & $-0,020$ & $-0,018$ \\
\hline Zuwanderung nach Geburt & & & & & 0,048 & 0,016 & 0,018 \\
\hline \multicolumn{8}{|l|}{ Schulrelevante Kompetenzen } \\
\hline Bewältigung des Schulalltags & & & & & & & $-0,001$ \\
\hline \multicolumn{8}{|l|}{ Sozialverhalten (Ref.: Unauffällig) } \\
\hline Grenzwertig & & & & & & & $0,047 * *$ \\
\hline Auffällig & & & & & & & $0,073 * * *$ \\
\hline$\overline{R^{2}}$ & 0,062 & 0,105 & 0,120 & 0,180 & 0,181 & 0,205 & 0,213 \\
\hline Beobachtungen & 3.850 & 3.850 & 3.850 & 3.850 & 3.850 & 3.850 & 3.850 \\
\hline
\end{tabular}

Anmerkungen: ${ }^{1}$ Abhängige Variable: Haupt-/Förderschulbesuch vs. Realschule/Gymnasium/Gesamtschule; Average Marginal Effects (AME); Alle Modelle kontrollieren für Region + Interviewperson.

Signifikanz: $* * * p<0.001 ; * * \mathrm{p}<0.01 ; * \mathrm{p}<0.05 ; \sim \mathrm{p}<0.10$; Eigene Analysen auf Basis des KiGGS (2003-2006). 
Insgesamt bleibt die Mediation bildungspositioneller Disparitäten über prä- und postnatale Einflüsse hinter den Erwartungen zurück. Erst nach Kontrolle sozioökonomischer und demographischer Merkmale lässt sich ein substanzieller Rückgang im Ausmaß ethnischer Schulbesuchsnachteile feststellen (M20). Für die türkischstämmige Sekundarschülerschaft lässt sich allein über die soziale Herkunft 75 Prozent der positionellen Ausgangsdifferenz (in M17) erklären. Positionsnachteile der Spätaussiedlerkinder werden vollständig über soziale Pfade vermittelt. Noch verbleibende Residualeffekte verringern sich jedoch für beide Gruppen, wenn Sprachlerngelegenheiten und Zuwanderungszeitpunkt kontrolliert werden (M21). Werden entwicklungsbiologische und soziokulturelle Erklärungsstränge zusammengeführt, ergibt sich kein nennenswerter zusätzlicher Erklärungsbeitrag (M22). Erst nach Berücksichtigung der Bewältigung des Schulalltags und des Verhaltens (wobei nur letzteres Merkmal mit dem Schulbesuch assoziiert ist) schwächt sich der positionelle Abstand merklich ab (M23). Wie im Methodenabschnitt dargelegt, wird für die Analysen ergänzend ein alternatives Schulerfolgsmaß verwendet. Dieses unterscheidet zwischen dem Besuch der Haupt-/Förderschule und der Realschule/Gymnasium, d.h. die Gesamtschülerschaft wird von den Analysen ausgeschlossen. Wie die Regressionskoeffizienten der Vergleichsmodelle (siehe Tabelle A 7.2, Anhang) zeigen, legen die ähnlichen Befunde keine abweichenden Interpretationen nahe.

\subsection{Diskussion}

Im Beitrag wurde der Möglichkeit nachgegangen, dass Bildungserfolg bereits während der Schwangerschaft vorstrukturiert wird. Für Deutschland liegen kaum Forschungsarbeiten zu diesem Thema vor, obgleich die These einer intrauterinen Prägung des Bildungserwerbs nicht neu ist. Für Nachkommen aus türkischen und Spätaussiedlerfamilien wurde geprüft, ob zwischen der entwicklungsbezogenen Ausgangslage (gemessen über die Dauer der Schwangerschaft, Geburtsgewicht und postnatale Gesundheit) und der Schulfähigkeit, den Grundschulnoten und dem Sekundarschulbesuch systematische Relationen bestehen. Die Analysen basierten auf dem Kinder- und Jugendgesundheits-Survey (KiGGS 2003-2006).

Für den Reproduktionserfolg zeigt sich: Kinder aus Familien unterer Sozialschichten, darunter vor allem türkische Familien, haben ein höheres Frühgeburtsrisiko. Dieser Befund ist nicht neu (RKI 2008; Razum et al. 2011). Für diese Gruppe besteht zudem eine leicht erhöhte Wahrscheinlichkeit für gesundheitliche Probleme nach der Geburt (David und Pachaly 2010: 26). In der Tendenz gilt dies auch für ein geringes Geburtsgewicht. Spätaussiedlerkinder werden hingegen nur etwas früher als deutsche Kinder geboren und haben tendenziell sogar ein höheres Geburtsgewicht. Diese Befunde berichten auch von Milewski und Peters (2014) auf Basis von SOEP-Daten. Spätaussiedlernachkommen haben zudem seltener gesundheitliche Probleme nach der Geburt. In den Analysen können diese Vorteile auf den Zuwanderungszeitpunkt zurückgeführt werden (siehe AMEs für Spätaussiedlerkinder in den Modellen M1c und M2c,Tabelle A 7.1), was auf den Healthy Migrant-Effekt hinweist (Janevic et al. 2011, für osteuropäische Frauen in den USA), dem zufolge vor allem gesunde Spätaussiedlerfamilien mit gesunden Nachkommen zuwandern. 
Differenzen im Schwangerschaftsergebnis zu Ungunsten von Migrantinnen lassen sich in Übereinstimmung mit dem Forschungsstand primär sozioökonomisch und/oder kulturellverhaltensbezogen erklären. Die Einflussgewichte dieser Faktoren variieren dabei je nach Kriterium teils erheblich. Relativ stabil sind die Assoziationen zwischen Vorsorgeneigung und Schwangerschaftsdauer sowie dem Geburtsgewicht: Je höher die Vorsorgeneigung, desto unwahrscheinlicher sind eine Frühgeburt oder niedriges Geburtsgewicht. Allerdings musste aufgrund von Datenbeschränkungen auf das Vorsorgeverhalten nach der Geburt als Proxy für das Vorsorgehandeln während der Schwangerschaft zurückgegriffen werden, was eine Verallgemeinerung dieses Ergebnisses einschränkt. Rauchen während der Schwangerschaft ist hingegen nur mit dem Geburtsgewicht, nicht aber mit der Frühgeburt oder postnatalen Gesundheitsproblemen assoziiert. Das Sprachkapital ist für den Reproduktionserfolg statistisch nicht bedeutsam, wobei sich auch hier das Problem ergibt, dass keine Informationen zu Sprachgebrauch und Sprachkompetenzen für die Phase der Schwangerschaft vorliegen. Auch die Ressourcenmerkmale wurden (weit) nach der Geburt erhoben, was die eher schwachen Effekte auf den Reproduktionserfolg erklären dürfte.

Mit Blick auf die Frage, wie Kinder mit Migrationshintergrund schulisch abschneiden und inwieweit die beobachteten Differenzen zu Kindern ohne familiäre Migrationsbiographie neben sozioökonomischen auch vorschulischen und vorgeburtlichen Faktoren zurechenbar sind, zeigen sich folgende Untersuchungsbefunde.

Zum Grundschuleintritt fallen Türkischstämmige, nicht aber Spätaussiedlerkinder, durch höhere Rückstellungsraten auf. Dieser Befund ist für die türkische Gruppe schon mehrfach berichtet worden (z. B. Tuppat und Becker 2014). Die Analysen bestätigen negative Effekte einer zu kurzen Schwangerschaftsdauer, postnataler Gesundheitsprobleme und des Rauchens während der Schwangerschaft auf die Schulfähigkeit, was erwartungsgemäß ist. Entgegen der Annahmen hängen eine geringe Präventionsneigung (Teilnahme an den U-Untersuchungen) und ein später Besuch einer Kindertagesstätte nur tendenziell, nicht aber statistisch signifikant mit einer höheren Rückstellungswahrscheinlichkeit zusammen. Nach Berücksichtigung schwangerschafts- und investitionsbezogener Merkmale schwächen sich die Nachteile beim Schuleintritt zwar ab, nahezu vollständig aufgeklärt werden diese aber nur durch die soziale Schichtzugehörigkeit. Bemerkenswert ist, dass bei gleichem sozialen Hintergrund Schwangerschaftsdauer und Säuglingsgesundheit höchstsignifikant mit der Zurückstellung vom Schulbesuch zusammenhängen. Dieser Befund unterstützt die These einer intrauterinen Vorstrukturierung des vorschulischen Kompetenzerwerbs.

Mit Blick auf den Schulerfolg zeigen sich folgende Ergebnisse: Kinder deutscher Herkunft positionieren sich am oberen, türkischstämmige Kinder am unteren Ende des Noten- und Positionsspektrums; Spätaussiedlerkinder nehmen mittlere Ränge ein - ebenfalls ein gut dokumentierter Befund (Gresch und Becker 2010). Hauptannahme war, dass sich Noten- und Bildungsdisparitäten auf schwangerschaftsbezogene, vorschulische und soziokulturelle Faktoren und Investitionen zurückführen lassen. Wie für die Schulfähigkeit, resultieren aus vorgeburtlichen Umständen und nachgeburtlichen Kulturationsanstrengungen schulische Erträge in 
Gestalt eines Noten- und Bildungsvorsprungs. Regelmäßiges Rauchen während der Schwangerschaft, eine geringe Vorsorgeneigung und (in abgeschwächter Form auch) die Frühgeburt hängen negativ mit der Grundschulnote im Fach Deutsch und dem Besuch einer weiterführenden Schule zusammen.

Nach Kontrolle schwangerschaftsbezogener Größen und Investitionen schwächen sich die Noten- und Bildungsdifferenzen zwischen den Herkunftsgruppen ab, eine vollständige Mediation bleibt aber unerreicht. In diesem Zusammenhang ist der soziale Hintergrund erklärungskräftiger, obgleich notenbezogene Schwierigkeiten türkischstämmiger Kinder auch hierüber nicht restlos aufgeklärt werden, was im Einklang mit bisherigen Studien ist (Stanat et al. 2010: 221; Kristen 2002). Im Unterschied dazu sind nach Einbezug perinataler Merkmale die spezifischen Nachteile der türkischstämmigen Grundschülerschaft nicht mehr signifikant und schwächen sich nach Kontrolle der Schichtzugehörigkeit sowie lern- und verhaltensbezogener Merkmale ab.

Die Abhängigkeit des Schulerfolgs vom Sozialverhalten zeigt, dass auch leistungsfremde Faktoren schulischen Sanktionen unterliegen. Für Kinder aus Migrantenfamilien, welche nach den Elternangaben häufiger ein grenzwertiges oder auffälliges Verhalten zeigen, liegt die schulische Erfolgshürde somit noch ein Stück höher. Idealerweise wären statt bzw. in Ergänzung zur Elternsicht auch Angaben der Lehrkräfte zum Verhalten gewesen, um die These zur Verletzung von Normalitätserwartungen im Unterricht besser testen zu können. Für Spätaussiedlerkinder zeigen sich nach Drittvariablenkontrolle keine besseren Noten (gegenüber Einheimischen), dafür aber marginale nicht signifikante Positionierungsvorteile im Sekundarschulsystem.

Weiterhin wurde angenommen, dass sich für Migrantenkinder an der selektiven Schwelle zur Grundschule langfristige schulische Nachteile ergeben. Die Analysen zeigen, dass das Einschulungsergebnis tatsächlich mit schulischem Misserfolg assoziiert ist, die Nachteile türkischstämmiger Kinder werden aber nicht, wie zum Beispiel von Gomolla und Radtke (2002) angenommen, über die Praxis der Zurückstellung vermittelt. Dennoch gibt der Zusammenhang zwischen Zurückstellung und Schulmisserfolg Anlass zur Diskussion der schulischen Effizienz und sozialen Gerechtigkeit von Schuleingangsselektionen.

Des Weiteren wurde die Vermutung überprüft, dass der Bildungserwerb entscheidend vom (mütterlichen) Risikohandeln (Rauchen) während der Schwangerschaft abhängt. Es zeigen sich stabile und starke Effekte des Rauchens auf die Schulfähigkeit und den Schulerfolg. Dies verweist auf eine frühe Manifestierung kognitiver Nachteile, deren Ursprung in der embryonalen und fötalen Lebensphase zu verorten ist und im Vorschulalter nur begrenzt kompensiert werden, zumal die Analysen ergeben, dass beide Migrantengruppen weniger präventiv handeln und formelle Kinderbetreuung weniger und später in Anspruch nehmen. Auch spielt die Nutzung der U-Untersuchungen und der formellen Kinderbetreuung für den Schulerfolg eine bedeutsame Rolle. Ethnische Bildungsdifferenzen werden über diese institutionellen Förderprogramme jedoch nur in geringem Ausmaß vermittelt. 
Zusammenfassend ist festzuhalten: Phänomene der ethnischen Ungleichheit zeigen sich schon zum Lebensbeginn und schlagen sich besonders in Gestalt eines höheren Risikos der Rückstellung vom Schulbesuch, und in stark abgeschwächter Form, auch in Gestalt notenund bildungspositioneller Nachteile nieder. Deshalb kann durchaus konstatiert werden, dass es auf den Anfang ankommt (Bourdieu 1983: 187), welcher aber nicht nur an den Umständen für die postnatale Bildungsaneignung festzumachen ist, sondern auch an vorgeburtlichen Einflüssen - auch bei gleichem sozialen Hintergrund. Dieser Befund stützt kumulationstheoretische Überlegungen, gemäß denen frühe Entwicklungsnachteile und geringe Investitionen signifikant die Schulfähigkeit und den späteren Bildungswettbewerb beeinträchtigen. Allerdings sind aufgrund der geringen Fallzahl der Migrantenpopulation und der genannten Datenbeschränkungen Replikationen durch weitere Studien nötig. 
Anhang

Tabelle A 7.1: $\quad$ Lineare Regression der Mathematiknote (GrundschülerInnen)

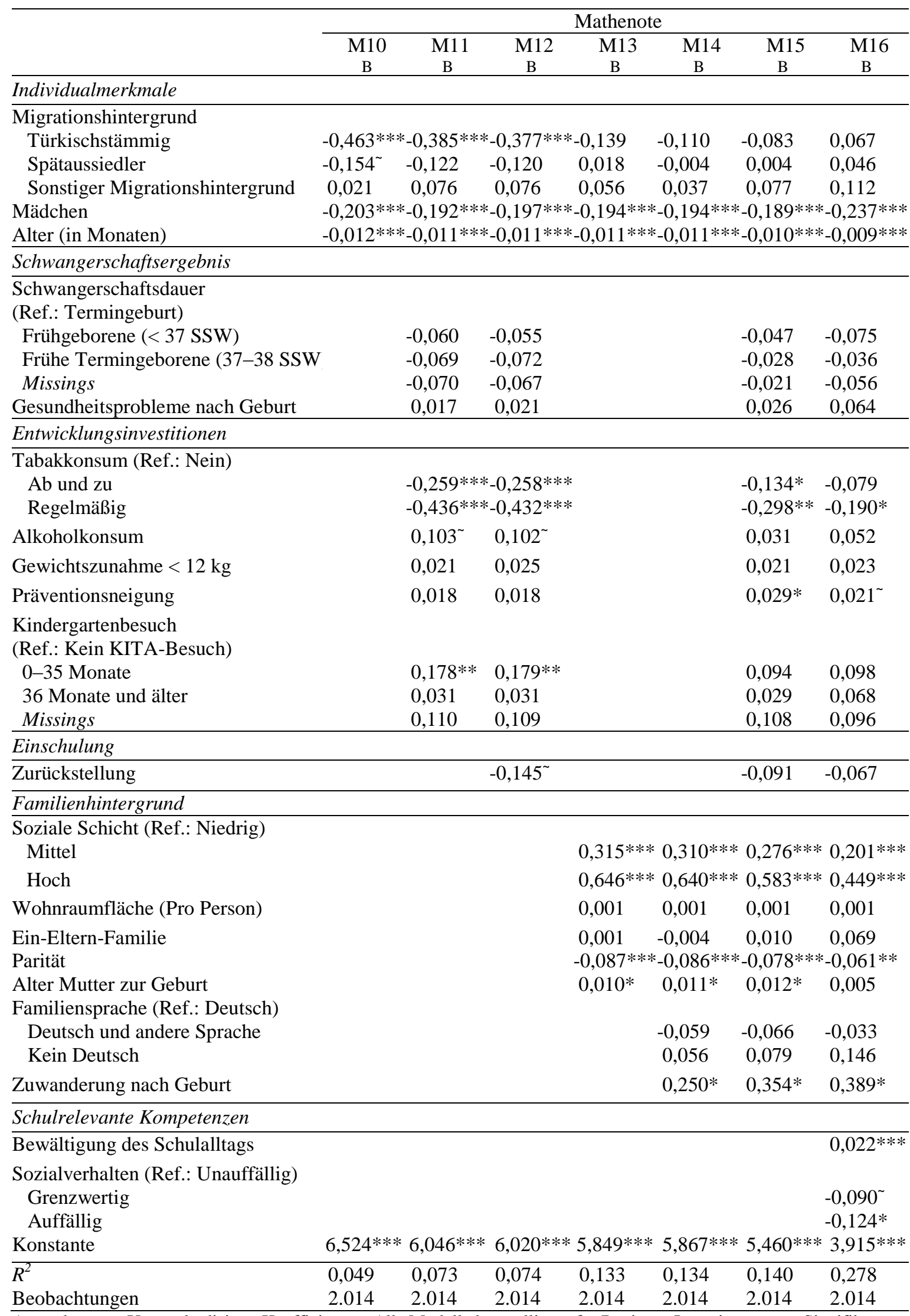

Anmerkungen: Unstandardisierte Koeffizienten; Alle Modelle kontrollieren für Region + Interviewperson. Signifikanz: $* * * \mathrm{p}<0.001 ; * * \mathrm{p}<0.01 ; * \mathrm{p}<0.05 ; \sim \mathrm{p}<0.10$; Eigene Analysen auf Basis des KiGGS (2003-2006). 
Tabelle A 7.2: $\quad$ Logistische Regression des Schulbesuchs (SekundarschülerInnen, ohne Gesamtschülerschaft)

\begin{tabular}{|c|c|c|c|c|c|c|c|}
\hline & \multicolumn{6}{|c|}{ Haupt-/Förderschulschulbesuch $^{1}$} & \multirow[b]{2}{*}{$\begin{array}{l}\text { M23a } \\
\text { AME }\end{array}$} \\
\hline & $\begin{array}{l}\text { M17a } \\
\text { AME }\end{array}$ & $\begin{array}{l}\text { M18a } \\
\text { AME }\end{array}$ & $\begin{array}{l}\text { M19a } \\
\text { AME }\end{array}$ & $\begin{array}{l}\text { M20a } \\
\text { AME }\end{array}$ & $\begin{array}{l}\text { M21a } \\
\text { AME }\end{array}$ & $\begin{array}{l}\text { M22a } \\
\text { AME }\end{array}$ & \\
\hline \multicolumn{8}{|l|}{ Individualmerkmale } \\
\hline \multicolumn{8}{|l|}{ Migrationshintergrund } \\
\hline Türkischstämmig & $0,254 * * *$ & $0,202 * * *$ & $0,197 * * *$ & $0,067 *$ & 0,053 & 0,048 & 0,042 \\
\hline Spätaussiedler & $0,075 * *$ & 0,039 & 0,041 & $-0,018$ & $-0,046$ & $-0,037$ & $-0,051$ \\
\hline Sonstiger Migrationshintergrund & $0,086 * * *$ & $0,043^{\sim}$ & $0,044^{*}$ & $0,045^{*}$ & 0,032 & 0,020 & 0,007 \\
\hline Mädchen & $-0,090 * * *$ & $-0,090 * * *$ & $-0,083 * * *$ & $-0,085 * * *$ & $-0,086 * * *$ & $-0,079 * * *$ & $-0,070 * * *$ \\
\hline Alter (in Monaten) & $0,002 * * *$ & $0,001 * * *$ & $0,001 * *$ & $0,001 * *$ & $0,001 * * *$ & $0,001 *$ & $0,001^{\sim}$ \\
\hline \multicolumn{8}{|l|}{ Schwangerschaftsergebnis } \\
\hline \multicolumn{8}{|l|}{$\begin{array}{l}\text { Schwangerschaftsdauer } \\
\text { (Ref.: Termingeburt) }\end{array}$} \\
\hline Frühgeborene $(<37$ SSW $)$ & & $0,053^{\sim}$ & 0,044 & & & $0,052^{\sim}$ & $0,056^{*}$ \\
\hline Frühe Termingeborene (37-38 SSW) & & 0,020 & 0,016 & & & 0,003 & $-0,003$ \\
\hline Missings & & $0,048 *$ & $0,047 *$ & & & 0,026 & 0,003 \\
\hline Gesundheitsprobleme nach Geburt & & $-0,014$ & $-0,019$ & & & $-0,003$ & $-0,006$ \\
\hline \multicolumn{8}{|l|}{ Entwicklungsinvestitionen } \\
\hline \multicolumn{8}{|l|}{ Tabakkonsum (Ref.: Nein) } \\
\hline $\mathrm{Ab}$ und $\mathrm{zu}$ & & $0,151 * * *$ & $0,148 * * *$ & & & $0,083 * * *$ & $0,072 * * *$ \\
\hline Regelmäßig & & $0,245^{* * *}$ & $0,235 * * *$ & & & $0,120 * * *$ & $0,105 * * *$ \\
\hline Alkoholkonsum & & $-0,045^{*}$ & $-0,042 *$ & & & $-0,015$ & $-0,017$ \\
\hline Gewichtszunahme $<12 \mathrm{~kg}$ & & $0,026^{\sim}$ & 0,023 & & & 0,017 & 0,018 \\
\hline Präventionsneigung & & $-0,014 * * *$ & $-0,013 * * *$ & & & $-0,008 * *$ & $-0,008 *$ \\
\hline \multicolumn{8}{|l|}{$\begin{array}{l}\text { Kindergartenbesuch } \\
\text { (Ref.: Kein KITA-Besuch) }\end{array}$} \\
\hline 0-35 Monate & & $-0,078 * *$ & $-0,075^{* *}$ & & & $-0,014$ & $-0,020$ \\
\hline 36 Monate und älter & & $-0,022$ & $-0,026$ & & & $-0,013$ & $-0,019$ \\
\hline Missings & & 0,022 & 0,029 & & & 0,026 & 0,015 \\
\hline \multicolumn{8}{|l|}{ Einschulung } \\
\hline Zurückstellung & & & $0,163 * * *$ & & & $0,136 * * *$ & $0,132 * * *$ \\
\hline \multicolumn{8}{|l|}{ Familienhintergrund } \\
\hline \multicolumn{8}{|l|}{ Soziale Schicht (Ref.: Niedrig) } \\
\hline Mittel & & & & $-0,139 * * *$ & $-0,139 * * *$ & $-0,114 * * *$ & $-0,107 * * *$ \\
\hline Hoch & & & & $-0,354 * * *$ & $-0,355 * * *$ & $-0,318 * * *$ & $-0,305 * * *$ \\
\hline Wohnraumfläche (pro Person) & & & & $-0,002 * *$ & $-0,002 * *$ & $-0,002 * *$ & $-0,002 *$ \\
\hline Ein-Eltern-Familie & & & & 0,024 & 0,025 & 0,015 & 0,012 \\
\hline Parität & & & & $0,057 * * *$ & $0,057 * * *$ & $0,052 * * *$ & $0,051 * * *$ \\
\hline Alter Mutter zur Geburt & & & & $-0,009 * * *$ & $-0,009 * * *$ & $-0,008 * * *$ & $-0,008 * * *$ \\
\hline \multicolumn{8}{|l|}{ Familiensprache (Ref.: Deutsch) } \\
\hline Deutsch und andere Sprache & & & & & 0,018 & 0,015 & 0,020 \\
\hline Kein Deutsch & & & & & $-0,034$ & $-0,038$ & $-0,035$ \\
\hline Zuwanderung nach Geburt & & & & & 0,041 & 0,006 & 0,007 \\
\hline \multicolumn{8}{|l|}{ Schulrelevante Kompetenzen } \\
\hline Bewältigung des Schulalltags & & & & & & & $-0,001^{\sim}$ \\
\hline \multicolumn{8}{|l|}{ Sozialverhalten (Ref.: Unauffällig) } \\
\hline Grenzwertig & & & & & & & $0,050 * *$ \\
\hline Auffällig & & & & & & & $0,089 * * *$ \\
\hline$\overline{R^{2}}$ & 0,064 & 0,113 & 0,127 & 0,199 & 0,201 & 0,227 & 0,237 \\
\hline Beobachtungen & 3.474 & 3.474 & 3.474 & 3.474 & 3.474 & 3.474 & 3.474 \\
\hline
\end{tabular}

Anmerkungen: ${ }^{1}$ Abhängige Variable: Haupt-/Förderschulbesuch vs. Realschule/Gymnasium; Average Marginal Effects (AME); Alle Modelle kontrollieren für Region + Interviewperson.

Signifikanz: $* * * p<0.001 ; * * \mathrm{p}<0.01 ; * \mathrm{p}<0.05 ; \sim \mathrm{p}<0.10$; Eigene Analysen auf Basis des KiGGS (2003-2006). 


\section{Diskussion und Zusammenfassung}

Die Arbeit widmete sich theoretisch und empirisch den Ursachen herkunftsspezifischer Ungleichheiten in drei sozialpolitisch bedeutsamen Dimensionen: Säuglingsgesundheit, Vorsorgeverhalten (Besuch der U-Untersuchungen für Kinder) und Erfolg im Bildungssystem.

Im Mittelpunkt der ersten Fragestellung standen gesellschaftliche sowie sozialmedizinische Sachverhalte, welche zur Entstehung von Gesundheitsdisparitäten zwischen Neugeborenen aus verschiedenen sozialen Gruppen beitragen. Die zweite Fragestellung zielte darauf, soziale Bedingungen zu identifizieren, die dem Bildungsgradienten im Vorsorgestatus Neugeborener zugrundeliegen. Gegenstand der dritten Fragestellung waren soziale und ethnische Bildungsdifferenzen. Hier galt es herauszufinden, ob Bildungsungleichheiten neben den gut dokumentierten Gruppenunterschieden in der Verfügbarkeit lern- und schulerfolgsrelevanter Ressourcen auf Unterschiede in der entwicklungsbezogenen Ausgangslage zur Geburt sowie auf Herkunftsunterschiede im Vorsorgehandeln zurückgeführt werden können. Im Fokus dieses interdisziplinären Forschungsdesiderats, auf das aktuell die empirische Bildungsforschung im Kontext mit dem teils unbefriedigenden Kenntnisstand zu den Gründen herkunftsspezifischer Entwicklungs-, Kompetenz- und Schulleistungsunterschiede (primäre soziale und ethnische Effekte der familiären Herkunft) aufmerksam macht (Kristen 2008), steht die Forschungsthese einer biographisch frühen mit der Schwangerschaft einsetzenden Vorstrukturierung lebensanfänglicher Gesundheits- und späterer Bildungschancen (Jackson 2013).

Für Deutschland wurden lebensanfängliche Ungleichheitsprozesse vor allem mangels geeigneter Datenquellen bisher nur in wenigen Studien untersucht (Wolke und Meier 2000; Jäkel et al. 2013). Daher fußt das Wissen um Faktoren, welche die Entstehung herkunftsspezifischer Ungleichheiten unter Neugeborenen und damit assoziierter Ungleichheiten in den Bildungschancen begünstigen, auf einer schmalen sowie in der Regel empirisch nicht bundesweit repräsentativen Grundlage. Ebenso liegen bislang nur wenige Studien zum elterlichen Präventionsverhalten in der frühen Kindheit vor (Kurz und Becker 2017). In der vorliegenden Arbeit galt es daher auch die Lücken zur Erklärung sozialer Unterschiede im Besuch der Früherkennungsuntersuchungen für Kinder zu schließen.

Die ausgewählten Forschungssprobleme wurden aus einer ressourcen- und lebenslauftheoretischen Perspektive betrachtet. Hierfür wurde einerseits Boudon's Konzept der primären und sekundären sozialen Herkunftseffekte herangezogen, wobei die multiplen wie komplexen Pfade, über welche die Familie Einfluss auf die Gesundheits- und Bildungschancen von Kindern nimmt, mithilfe von Bourdieu's Kapital- und Habitustheorie weiter spezifiziert wurden. Nach Bourdieus körpersoziologischer Formel Exposition gleich Disposition wurde angenommen, dass primäre soziale Herkunftseffekte in der Gesundheit und Bildung aus einer vorwie nachgeburtlich ungleichen Exposition zu Einflüssen der Sozialwelt resultieren.

In diesem Kontext wurde die Aufmerksamkeit einerseits auf ressourcenbezogene Expositionen gelenkt, welche von Geburt an die kindliche Entwicklung entscheidend mitprägen. In Erweiterung bzw. in Ergänzung zur klassischen Ressourcen- und Sozialisationsperspektive 
wurde unter Berücksichtigung neuerer Erkenntnisse der sozialepidemiologischen Lebenslaufforschung das Zeitfenster zur Betrachtung sich ungleich vollziehender Entwicklungsprozesse auf den für den ungeborenen Nachwuchs äußerst kritischen vorgeburtlichen Lebensabschnitt ausgeweitet. Dies schien angebracht angesichts älterer wie neuer Evidenzen (Mielck 2005), welche zeigen, dass die Weichen für eine gute Neugeborenengesundheit zu einem bedeutsamen Teil schon vor der Geburt gestellt werden - kraft ökonomischer, kultureller und sozialer Ressourcen des Elternhauses, deren strukturierende Wirkung auf die Gesundheit Neugeborener auch im Zusammenhang mit einer für die intrauterine Entwicklung wichtigen gesundheitsorientierten Lebensführung schwangerer Frauen zu verstehen sind.

Vor diesem Hintergrund wurde mit den Modellen kritischer Entwicklungsperioden und der Akkumulation individueller Vor- und Nachteile im Lebenslauf angenommen, dass Entwicklungsbeeinträchtigungen bei Neugeborenen vorschulische Entwicklungsprozesse negativ beeinflussen. Ergebnis dieser Prozesse, so die Vermutung (siehe Erklärungsmodell in Kapitel 7), ist eine nach familiärer Herkunft variierende kompetenzbezogene Ausgangslage zur Einschulung, welche wiederum den Notenerfolg in der Grundschule und somit die Bildungspositionierung in der Sekundarstufe mitstrukturiert.

Um empirisch die Rolle vor- und nachgeburtlicher Prozesse für die Entstehung ungleicher Gesundheits- und Bildungschancen zu untersuchen, bedarf es geeigneten statistischen Materials, das neben Merkmalen zu sozialem Hintergrund (elterliche Ressourcenlage), ethnischer Herkunft (Geburtsland Eltern und Kind) und Mutter-Kind-Beziehung idealerweise Informationen zur gesundheitlichen Lebensführung während der Schwangerschaft (Substanzkonsum), Präventionsverhalten (Teilnahme an Vorsorgeuntersuchungen), Geburtsergebnis (Früh- vs. Termingeburt, Geburtsgewicht, postnatale Gesundheit Neugeborener) sowie zu verschiedenen Bildungs- und Schulerfolgskennziffern (Einschulungsergebnis, Fachnoten, Bildungspositionierung) und Informationen zur individuellen Bewältigung des Schulalltags beinhaltet.

Diese für die Beantwortung der hier untersuchten Forschungsfragen zentralen Variablen wurden erst in jüngster Zeit durch sozial- und gesundheitswissenschaftliche Surveys bereitgestellt. So verbesserte sich die zumeist nur kleinräumige regionale Untersuchungen erlaubende Forschungsdatenlage zu Beginn der 2000er Jahre erheblich durch die Zusatzerhebung von Informationen zu Gesundheit und Entwicklung von Neugeborenen, Klein-, Vorschul- und Schulkindern im Sozioökonomischen Panel (Mutter-Kind-Fragbögen, seit 2002) sowie den neu ins Leben gerufenen bundesweit repräsentativen Kinder- und Jugendgesundheitssurvey (Basiserhebung, 2003-3006), welcher umfangreiche Befragungs- und medizinische Untersuchungsdaten bereitstellt.

Datenbasis für die Beantwortung der Fragestellungen waren das Sozioökonomische Panel (Mutter-Kind-Fragebögen, Lebensalter 0 bis 1 Lebensjahr) sowie der Kinder- und Jugendgesundheitssurvey (Basiserhebung). Die hier in den Untersuchungsfokus gerückten Relationen zwischen sozialem und ethnischem Hintergrund auf der einen Seite und Gesundheits- und Bildungschancen der heranwachsenden Generation auf der anderen konnten mit den verwendeten Surveydaten relativ differenziert unter dem Herkunftsaspekt untersucht werden - trotz 
Dateneinschränkungen bezüglich der operationalen Abbildung der Einstellungsebene und fallzahlbezogener Begrenzungen für feingliedrige Subgruppenanalysen.

Nachfolgend werden die zentralen Untersuchungsbefunde zusammengefasst und diskutiert. Am Anfang stehen die Befunde zu den Ursachen gesundheitlicher Ungleichheiten unter Neugeborenen zur Diskussion (Abschnitt 8.1). Es folgen die Untersuchungsbefunde zu sozialen Vorsorgedisparitäten (Abschnitt 8.2). Im Anschluss werden die Ergebnisse zu den Bildungsauswirkungen gesundheitlicher Ungleichheiten zusammengefasst (Abschnitt 8.3). Nach der Ergebnisdarstellung und -diskussion werden schließlich die in Kapitel 2.5 abgeleiteten zentralen Arbeitshypothesen mit den in den Analysen gewonnenen Erkenntnissen abgeglichen (Abschnitt 8.4). Den Schlusspunkt der vorliegenden Arbeit bildet ein Fazit (Abschnitt 8.5).

\subsection{Gesundheitliche Ungleichheit zum Lebensbeginn}

Warum hängen eine geringe formale Bildung und ein Migrationshintergrund mit einem höheren Frühgeburtsrisiko zusammen? Diese Fragen galt es unter Rückgriff auf Bourdieus Kapital- und Habituskonzept zu beantworten. Beiden Strukturphänomenen wurde aufgrund von Datenbeschränkungen mit unterschiedlichen Surveydaten empirisch nachgegangen.

Mit Blick auf die erhöhten Frühgeburtsrisiken in der Gruppe formal gering gebildeter Frauen wurde vermutet, dass diese das Resultat einer im Mittel geringeren ökonomischen und sozialen Kapitalausstattung sowie herkunftsspezifischer Gesundheitsorientierungen und Gesundheitspraxen sind (siehe Kapitel 5). Multiple Regressionsanalysen auf Basis des Soziooekonomischen Panels (SOEP; Wellen 2003-2011) bestätigten die Bedeutsamkeit kultureller Ressourcen für das Geburtsergebnis. Frauen mit niedrigem Bildungsabschluss gebaren ihre Kinder häufiger vor Vollendung der 37. Schwangerschaftswoche und somit zu früh, verglichen mit Frauen mit weiterführenden Bildungsabschlüssen.

Im Gegensatz dazu waren die Indikatoren für ökonomisches Kapital (relative Armut) und soziales Kapital (Bestehen einer Partnerschaft, hohes Bildungskapital des Partners, Besuch durch Freunde und Bekannte) nur tendenziell mit zu kurzen Schwangerschaften assoziiert. Vergleichsweise stärkere Effekte auf die Frühgeburtlichkeit ließen sich dabei für das Risikoverhalten während der Schwangerschaft (Zigarettenkonsum) und der im Mittel geringer ausgeprägten allgemeinen Kontrollüberzeugung („Leben ist abhängig vom Glück“) beobachten. Während sich ersterer Befund in die umfangreiche Literatur zu verhaltensbedingten Schwangerschaftskomplikationen einreiht (Behrman und Butler 2007) und die Rolle der Exposition zu Umweltgiften und damit die Relevanz des Modells kritischer Entwicklungsperioden stärkt, weist der zweite Befund auf die Bedeutung habitustheoretischer Mechanismen bei der Entstehung nachteiliger Schwangerschaftsergebnisse hin. Sind Menschen der Überzeugung kaum Kontrolle über das eigene Leben zu besitzen, sehen sie sich, so die Theorie, selbst weniger in der Lage gesundheitsorientiert zu handeln. Die signifikante Assoziation dieses Denkschemas mit der Frühgeburtlichkeit deutet trotz der allgemeinen und nicht gesundheitsbezogenen Operationalisierung des Konstrukts auf die Relevanz verinnerlichter Handlungsdispositionen hin. Um diesen Gedankengang empirisch besser zu untermauern, wäre es hilfreich, für die statisti- 
sche Auswertung auf entsprechende Informationen zurückzugreifen. In Anbetracht der in dieser Hinsicht eingeschränkten Datenbasen müssen nachfolgende Untersuchungen mit adäquateren Daten den Beleg für oder gegen das Erklärungspotential gesundheitsbezogener Denk-, Wahrnehmungs- und Handlungsschemata erbringen. Nichtsdestotrotz stellt die Betrachtung der allgemeinen Kontrollüberzeugung eine Stärke der Untersuchung dar, da das zwischen sozialer Struktur und Praxis zwischengeschaltete Habitusglied nicht ausschließlich auf der interpretativen Ebene verhaftet blieb, sondern empirisch kontrolliert wurde. Welches Ergebnis erbrachte nun die Auswertung bezüglich der zentralen Fragestellung?

Nach gemeinsamer Kontrolle aller ressourcen- und kulturell-verhaltensbezogenen Erklärungsfaktoren verlor der untersuchte Zusammenhang zwischen Bildung und Frühgeburt erwartungsgemäß an statistischer Bedeutsamkeit; eine vollständige Aufklärung dieses sozialen Ungleichheitsphänomens leisteten die multivariaten Regressionsmodelle aber nicht. Das kann, wie in Kapitel 5.5 ausführlich diskutiert, methodischen Einschränkungen bei der Operationalisierung zentraler theoretischer Konstrukte - wie kulturelles oder soziales Beziehungskapital - Fallzahlproblemen oder einer Unterspezifizierung relevanter erklärender Mechanismen geschuldet sein. Einige dieser Einschränkungen sollen an dieser Stelle benannt und diskutiert werden. Hinsichtlich der Messung des elterlichen Kulturkapitals wäre neben dem Bildungsabschluss wenigstens ein Indikator zum objektiven Kulturkapital wünschenswert gewesen. Noch besser wäre die Abbildung gesundheitskultureller Wissensbestände rund um die Thematik Schwangerschaft und Geburt sowie die Erhebung der gesundheitsbezogenen Risikowahrnehmung der (werdenden) Eltern, einschließlich ihrer subjektiven Relevanz präventiv zu handeln.

Mit Blick auf die Messung des sozialen Beziehungskapitals empfiehlt sich des Weiteren, neben den datenbedingt vorgegebenen quantitativen Kapitaldimensionen zusätzlich qualitative Maße zur sozialen Einbindung werdender Mütter zu berücksichtigen, wie etwa die erlebte Qualität der Paarbeziehung und die Zufriedenheit mit familiären, freundschaftlichen und nachbarschaftsbezogenen Sozialkontakten. In Bezug auf die Berücksichtigung alternativer sowohl potentiell negativ auf den Verlauf von Schwangerschaften und Geburtsergebnissen wirkende als auch mit einem formal geringeren Bildungsabschluss zusammenhängenden Faktoren sind physische und mentale Belastungen im Zusammenhang mit dem Nachgehen einer Erwerbstätigkeit zu nennen. Zwar bietet das Sozioökonomische Panel (nicht aber die KiGGSDaten) die Möglichkeit, mit Paneldaten die Bindung schwangerer Frauen an den Arbeitsmarkt zu rekonstruieren, allerdings standen zum Zeitpunkt der durchgeführten Analysen erwerbstätigkeitsbezogene Schwangerschaftskomplikationen nicht im theoretischen Blickfeld.

Eine plausible Erklärung für Residualeffekte der formalen Bildung auf die Schwangerschaftsdauer könnte also am Arbeitsmarkt ansetzen. Bereits Engels (1845) diagnostizierte im 19. Jahrhundert einen Gesundheitsnotstand: Unter prekären Lebens- und Arbeitsbedingungen gebaren englische Fabrikarbeiterinnen stark untergewichtige Kinder. In modernen Gesellschaften sind solche extremen gesundheitsgefährdenden Umweltbedingungen eher selten. 
Zudem schützt der Gesetzgeber erwerbstätige Schwangere. ${ }^{70}$ Ein Aushebeln des sozialpolitischen Schutzmechanismus ist grundsätzlich aber dennoch denkbar, zum Beispiel infolge ökonomischen Zwangs, einer starken Karriereorientierung oder infolge der Befürchtung, bei längerem Ausfall den Arbeitsplatz zu riskieren. Empirisch spielen besonders physisch anstrengende manuelle Berufstätigkeiten eine bedeutsame - obgleich nicht alleinige (Gisselmann und Hemström 2008: 1297) - Rolle für die Genese herkunftsspezifischer Unterschiede im Frühgeburtsrisiko, Geburtsgewicht und einhergehend damit in der Säuglingsgesundheit (Mozurkewich et al. 2000). Dieser Erklärungspfad kann zukünftig mit dem SOEP untersucht werden.

Darüber hinaus können weiterführende Forschungen zur Relation zwischen Bildungskapital und Geburtsergebnis gesundheitsorientierte Verhaltensweisen schwangerer Frauen näher untersuchen. So legt der Stand der Forschung nahe, dass Schwangere aus unteren Bildungsschichten später und in einem geringeren Umfang an den angebotenen Vorsorgeuntersuchungen teilnehmen, wobei die sozial strukturierte Vorsorgedistanz, so die Befundlage, wiederum mit ungünstigen Geburtsergebnissen assoziiert ist (Razum et al. 2011). Im Rahmen der verwendeten Datenbasen konnte bedauerlicherweise nicht auf adäquate schwangerschaftsbezogene Vorsorgeinformationen zurückgegriffen werden. Behelfsweise kam deshalb ein mit den SOEP-Daten konstruiertes Vorsorgemaß zum Einsatz (siehe Methodenteil in Kapitel 5), das jedoch empirisch nicht bedeutsam mit der abhängigen Frühgeburtsvariablen assoziiert war. Da es sich hierbei um ein statistisches Konstrukt handelt, ist dieser Befund jedoch mit Vorsicht zu interpretieren. Vorsicht scheint auch mit Blick auf die Verallgemeinerung der Befunde zur ethnischen Strukturierung der Schwangerschaftsergebnisse geboten, da die theoretischen Konstrukte datenbedingt nicht durchweg befriedigend in valide Indikatoren überführt werden konnten und darüber hinaus die nach ethnischen Herkunftsgruppen aufgeschlüsselten Analysen an fallzahlbezogene Grenzen stießen.

Welche Erkenntnisse förderten die Analysen hinsichtlich ethnisch strukturierter RegelmäBigkeiten in den Geburtsergebnissen zu tage? Das hier interessierende regel- und dauerhafte Muster bezog sich darauf, dass in Deutschland - wie in anderen europäischen Ländern (vgl. Bollini et al. 2008) - Frauen mit direktem und indirekten Migrationshintergrund im Vergleich zur Mehrheitsbevölkerung ohne Migrationshintergrund mitunter höhere Frühgeburtsrisiken haben. Aufgrund von Datenbeschränkungen liegt für den deutschen Forschungsraum zu Ausmaß und Ursachen dieser Phänomene bislang wenig Wissen vor (Razum et al. 2011). Dabei erschwert insbesondere die oftmals geringe Größe der verfügbaren Datenbasen eine differenzierte Betrachtung einzelner Migrantengruppen (Becker und Stolberg 2013). Die Arbeit rückte deshalb unter Rückgriff auf die vergleichsweise große Datenbasis des Kinder- und Ju-

\footnotetext{
${ }^{70}$ Das Mutterschutzgesetz (MuSchG), § 3 (Beschäftigungsverbote für werdende Mütter, Abs. 1 und 2) besagt im Wortlaut: (1) Werdende Mütter dürfen nicht beschäftigt werden, soweit nach ärztlichem Zeugnis Leben oder Gesundheit von Mutter oder Kind bei Fortdauer der Beschäftigung gefährdet ist. (2) Werdende Mütter dürfen in den letzten sechs Wochen vor der Entbindung nicht beschäftigt werden, es sei denn, dass sie sich zur Arbeitsleistung ausdrücklich bereit erklären; die Erklärung kann jederzeit widerrufen werden.
} 
gendgesundheitssurveys die zwei größten in Deutschland lebenden Migrantengruppen in den Untersuchungsfokus: Genauer wurde untersucht, ob türkischstämmige Frauen oder Spätaussiedlerinnen im Vergleich zu Schwangeren ohne Wanderungsbiographie eher verfrüht, also noch vor Vollendung der 37. Schwangerschaftswoche entbinden.

Die zuvor in erster Linie auf sozialbedingte Schwangerschaftskomplikationen angewendete kapitaltheoretische Einbettung (siehe Kapitel 5) wurde für die ungleichheitssoziologische Erklärung der ethnisch strukturierten Reproduktionsrisiken um migrationsspezifische sowie herkunftsspezifische (d.h. sozialmedizinische) Argumente erweitert (siehe Kapitel 7). Hierfür wurde einerseits auf die Bedeutsamkeit der gesellschaftlichen Kontextabhängigkeit gesundheitsrelevanter Ressourcen, wie etwa geburtslandspezifisches Wissen zum Vorsorgesystem und dessen wanderungsbedingte Entwertung im Aufnahmeland, abgestellt. Eine wanderungsbedingte Kapitalentwertung wurde darüber hinaus in Bezug auf die Herkunftssprache diskutiert, wobei die Kompetenz in der Verkehrssprache des Aufnahmelandes sowie Insitutionenvertrauen für die Inanspruchname offerierter (Vorsorge-)Dienste Relevanz besitzen. Andererseits wurde die Aufmerksamkeit auf herkunftsspezifische medizinische Ursachen (Größe, Alter, Parität) gelenkt. Letzteres war sinnvoll, da diese auf die mütterliche Konstitution abzielenden Merkmale nachweislich als erklärende Merkmale für ethnische (und soziale) Unterschiede in den Geburtsergebnissen gelten (siehe z.B. Razum et al. 2011). Mit multivariaten Analysemethoden wurde schließlich überprüft, ob sich ethnisch strukturierte Frühgeburtsdifferenzen über Gruppenunterschiede a) in der Ausstattung mit ökonomischen, kulturellen und sozialen Ressourcen, b) im Gesundheitshandeln während der Schwangerschaft (Rauchen) und c) in sozialmedizinischen Merkmalen (Konstitution) erklären lassen. Für die Analysen wurden multiple logistische Regressionen auf Basis der KiGGS-Daten geschätzt.

Mit den KiGGS-Daten ließen sich bedeutsame Unterschiede zwischen verschiedenen sozialen und ethnischen Gruppen in der Wahrscheinlichkeit einer Frühgeburt feststellen. Kinder aus Migrantenfamilien sowie aus Familien unterer Sozialschichten werden häufiger zu früh geboren. Obgleich weniger markant, zeigten sich für weitere Indikatoren zur allgemeinen Lebensfähigkeit Neugeborener strukturierte Muster, jedoch nicht durchweg in dieselbe Richtung weisend. So zeigt sich für das mittlere Geburtsgewicht: Je höher die Sozialschichtzugehörigkeit, desto schwerer sind Neugeborene. Ebenso haben Neugeborene aus höheren Schichten in den ersten Lebenswochen tendenziell weniger gesundheitliche Probleme. Diese Befunde waren ressourcentheoretisch zu erwarten und reihen sich in den empirischen Forschungsstand. Für Migrantenkinder stellt sich das Ungleichheitsbild zu diesen geburtsbezogenen Kennziffern jedoch wesentlich differenzierter dar als für das Merkmal Sozialschichtzugehörigkeit: Spätaussiedlerkinder wiegen direkt nach ihrer Geburt im Mittel etwas mehr als Neugeborene aus Nicht-Migrantenfamilien und schneiden hinsichtlich ihres postnatalen Gesundheitsstatus sogar besser ab. Demgegenüber starten türkischstämmige Kinder häufiger mit geringeren Chancen ins Leben: Ihr Geburtsgewicht liegt im Mittel unter dem der zwei Vergleichsgruppen. Dieses Ungleichheitsmuster wurde auch in früheren Untersuchungen mit SOEP-Daten beobachtet (Milewski und Peters 2014). Ebenfalls im Einklang mit bisherigen Evidenzen 
(z.B. Razum et al. 2011) steht der Befund, dass türkischstämmige Kinder eher postnatale Gesundheitsprobleme aufweisen (Tabelle 7.3).

Wie die Untersuchungsbefunde zeigen, stellen sich schwangerschaftsbezogene Unterschiede zwischen den betrachteten ethnischen Gruppen primär als soziales Ressourcenproblem dar, sekundär als Problem der gesundheitlichen Lebensführung und - zu einem geringen Teil - als Folge variierender sozialmedinischer Einflussfaktoren. In Bezug auf das Gesundheitshandeln gaben türkischstämmige Frauen gegenüber Frauen ohne Migrationshintergrund zwar häufiger an, während der Schwangerschaft nicht auf den Zigarettenkonsum verzichtet zu haben; dafür konsumierte diese Gruppe aber seltener Alkohol. Insgesamt konnten mit den KiGGS-Daten ethnische Differenzen in den Geburtsergebnissen fast vollständig über Gruppenunterschiede in der sozialen Ressourcenkomposition aufgeklärt werden (siehe Endmodelle in Kapitel 7). Im Gegensatz wurden schichtabhängige Frühgeburtsdifferenzen nur partiell aufgeklärt. Auch die Bildungsunterschiede in der Frühgeburtlichkeit konnten in den multiplen Regressionsanalysen auf Basis der SOEP-Daten nicht vollständig aufgeklärt werden (Modelle in Kapitel 5).

Insgesamt belegen die Ergebnisse relativ hohe Frühgeburtsrisiken für Migrantinnen, wobei diese bei Spätaussiedlerinnen im Vergleich zu türkischstämmigen Frauen geringer ausfallen. Erwartungsgemäß schwächten sich diese Unterschiede nach Kontrolle ressourcenbezogener sowie konstitutioneller Merkmale für beide Migrantengruppen ab. Unerwarteterweise leistete die Berücksichtigung des Risikoverhaltens keinen darüber hinaus gehenden Erklärungsbeitrag. Nach Berücksichtigung aller Erklärungsfaktoren bestanden keine signifikanten Frühgeburtsdifferenzen zwischen Migrantinnen und Nicht-Migrantinnen fort, ein Befund, der im Einklang mit den Ergebnissen einer von Becker und Stolberg (2013) durchgeführten SOEPStudie steht. Allerdings blieb in den Analysen mit den KiGGS-Daten, im Unterschied zur Analyse des Bildungsgradienten in der Schwangerschaftsdauer (Kapitel 5), die Habitusebene unberücksichtigt, da habitusbezogene Dimensionen nicht Teil des Erhebungsprogramms vom KiGGS sind. Inwiefern individuelle Kontrollüberzeugungen, Selbstwirksamkeitserwartungen und Gesundheitskonzepte bei Migrantinnen anders ausgeprägt sind als bei der Referenzgruppe der Nicht-Migrantinnen und inwiefern diese das Gesundheits- und Präventivhandeln anleiten und hierüber Relevanz für die Gesundheitschancen Neugeborener erlangen, blieb in der vorliegenden Arbeit somit ungeprüft. Mit dem SOEP können solche Fragestellungen in Analysen mit den bereitgestellten Indikatoren zu allgemeinen Kontrollüberzeugungen näherungsweise untersucht werden. Wünschenswert wäre in diesem Kontext, Fragen nach gesundheitsbezogenen Einstellungen und Dispositionen, zum Risikoverhalten (Rauchen, Alkohol- und Medikamentenkonsum während der Schwangerschaft) und Vorsorgeverhalten (kontinuierliche Nutzung der Schwangerenvorsorgedienste, Erhebung der Teilnahme an allen angebotenen Vorsorgeuntersuchungen für Kinder) in den Mutter-Kind-Fragebogen zu integrieren.

\subsection{Ungleichheiten im Vorsorgestatus Neugeborener}

Im Untersuchungsfokus der zweiten Forschungsfrage stand der Zusammenhang zwischen sozialer Herkunft und elterlichem Präventionsverhalten. Genauer interessierte die Erklärung 
der sozial differentiellen Inanspruchnahme der U-Untersuchungen für Kleinkinder bis zum Alter von 18 Monaten. Als erklärende Faktoren wurden die sozialen Beziehungen und zeitlichen Ressourcen der Mutter, einschließlich der Zufriedenheit mit der Mutterrolle und dem Zurechtkommen mit den rollenbezogenen Anforderungen betrachtet.

Auf Basis der Daten aus der Mutter-Kind-Befragung des SOEP (2003-2008) wurde mittels multipler logistischer Regressionsanalysen getestet, inwieweit die Einbindung in familiäre Beziehungen und die hier bereitgestellten Unterstützungsleistungen soziale Ungleichheitsmuster in der Teilnahme an einer altersgemäßen Vorsorgeuntersuchung erklären kann.

Die empirischen Analysen zeigten einen höchstsignifikanten Herkunftseinfluss auf das Teilnahmeverhalten in Abhängigkeit der Bildung - Je höher die formale Bildung der Eltern, desto eher nehmen Kinder an einer altersentsprechenden U-Untersuchung teil - ein bekannter Ungleichheitsbefund, der in anderen Studien mit für Deutschland repräsentativen Daten berichtet wurde (mit KiGGS-Daten, Kurz und Becker 2017). Daneben erwies sich ein Migrationshintergrund als bedeutsamer Teilnahmeprädiktor. Allerdings wurde dieser in Kapitel 6 nicht differenziert nach ethnischen Herkunftsgruppen betrachtet. Dafür ergab aber die Auswertung der KiGGS-Daten im Zusammenhang mit den Analysen zur dritten Fragestellung (Kapitel 7), dass Kinder aus türkischstämmigen und Spätaussiedlerfamilien einen vergleichsweise lückenhaften Vorsorgestatus aufweisen.

Auf theoretischer Ebene kann das mit der ethnischen Zugehörigkeit assoziierte Vorsorgehandeln über eine Vielzahl möglicher Einflussfaktoren in Verbindung gebracht werden, etwa mit mangelnden Kenntnissen zum Aufbau des Gesundheitsversorgungssystems, Einkommensarmut, einstellungsbezogene Präventionsdistanzen (fatalistischer Gesundheitshabitus), Sprachbarrieren oder Diskriminierung. Da ethnisch strukturiertes Vorsorgehandeln hier aber nicht als Explanandum sondern als Explanans (für herkunftsabhängige Bildungsunterschiede) in den Analysefokus gerückt wurde, können zu den zugrundeliegenden Teilnahmemechanismen keine Aussagen getroffen werden.

Welche Befunde zeigen nun die Analysen zu bildungsbezogenen Besuchsunterschieden? In Übereinstimmung mit den theoretischen Überlegungen fördert soziale Integration - gemessen über das Zusammenleben mit dem Kindsvater - maßgeblich die Teilnahmebereitschaft. Dieser familienstrukturelle Ungleichheitsbefund wurde wiederholt für den deutschen und internationalen Forschungsraum berichtet (Kurz und Becker 2017). Die Gründe dafür, warum es Kernfamilien gegenüber Ein-Elternformen besser gelingt, kontinuierlich gesundheitsbezogene Check-ups in Anspruch zu nehmen, wird aber im Allgemeinen noch unzureichend verstanden. Auch die hier vorgestellte Untersuchung wirft mit einem relativ begrenzten Bündel erklärender Größen nur wenig Licht auf die verantwortlichen Ursachen. Neben der strukturellen Vollständigkeit der Familie wirken das Wohlbefinden mit der Mutterrolle und die psychosoziale Gesundheit der Mutter während der Schwangerschaft, was sich auf theoretischer Ebene als Beziehungskapital des Kindes rahmen lässt, positiv auf das nach der Geburt erhobene Vorsorgeverhalten. Entgegen der Annahme, dass soziale Unterstützung im familiären Umfeld die Wahrscheinlichkeit des Besuchs einer altersgemäßen U-Untersuchungen erhöht, lässt sich 
in den Analysen jedoch kaum ein förderlicher Einfluss instrumenteller Unterstützung seitens des Partners (Kinderbetreuung) auf das Vorsorgehandeln erkennen. Umfangreiche verwandtschaftliche Unterstützungsleistungen bei der Kinderbetreuung sind mit dem Präventionsverhalten sogar negativ korreliert. Vermutlich verweist dieser abklärungsbedürftige Befund weniger auf die negative Seite sozialer Einbindung, als vielmehr auf eine (oder mehrere) in den Analysen unkontrollierte und damit unbeobachtete Kovariate(n). Beispielsweise ist denkbar, dass Kinder, welche in der Woche regelmäßig von Angehörigen, wie älteren Geschwistern, betreut werden, eher in Elternhäusern mit begrenzten Zeit- und/oder psychosozialen Ressourcen, die für präventives Engagement wichtig sind, aufwachsen. In den Analysen wurde zwar für diesbezügliche Indikatoren kontrolliert. Allerdings kann nicht mit Bestimmtheit gefolgert werden, dass die verwendeten (und durch die Daten vorgegebenen) Ressourcenmaße die Sozialkapitalkonstrukte adäquat abbilden, zumal in der Forschung kein Konsens darüber besteht, was unter dem Sozialkapitalbegriff zu verstehen und wie Sozialkapital zu messen ist (Franzen und Pointner 2007). Ungeachtet dieser sich fortsetzenden Sozialkapitaldiskussion zeigte sich in den Daten die präventive Relevanz der Gesundheitsressource Zeit: Mütter, welche sich zum Erhebungszeitpunkt in Mutterschutz, Mutterschaftsurlaub oder Elternzeit befinden, nahmen eher an den U-Untersuchungen im Rahmen des Krankheitsfrüherkennungsprogramms teil als einer Erwerbstätigkeit nachgehende Mütter.

Neben instrumententheoretischen Einschränkungen bezüglich der unabhängigen Variablen stellt die Operationalisierung der abhängigen Vorsorgevariablen einen Kritikpunkt dar. Wie die Befundlage zeigt (Kamtsiuris et al. 2007a), verliert das Vorsorgeprogramm mit fortschreitendem Lebensalter der Kinder (Stich et al. 2009) in allen Bildungsgruppen an Akzeptanz, wobei sich soziale Vorsorgedistanzen erst bei den späteren U-Untersuchungen (ab der U6, Kurz und Becker 2017: 179) sichtbar weiten. Es empfiehlt sich daher, die hier gemachten Beobachtungen an einem ,älteren' Kindersample zu replizieren, und dabei nicht, wie hier datenbedingt vorgegeben, mit Informationen zum letzten erfolgten Check-up, sondern idealerweise mit Informationen zur erfolgten Teilnahme an allen angebotenen Untersuchungen. Ein metrisches Maß zur Anzahl der Teilnahmelücken hat den Vorteil eines höheren Informationsgehaltes. Die KiGGS-Daten stellen ein solches Maß zur elterlichen Vorsorgeneigung bereit, dafür aber fehlen in der Basiserhebung Informationen zur Abbildung der Sozialkapitalmerkmale, wie der wahrgenommene Rückhalt, Hilfen bei der Kinderbetreuung und die Zufriedenheit mit der Mutterrolle und dem Zurechtkommen mit den rollenbezogenen Anforderungen.

Auch wenn insgesamt die Tragfähigkeit des Sozialkapitalkonzepts für die Erklärung sozialer Präventionsunterschiede begrenzt scheint, spielen die Vollständigkeit der Familie und die Gesundheitsressourcen der Mutter eine bedeutsame Rolle für die Nutzung der Gesundheitsuntersuchungen für Kinder. Eine Erklärung der Disparitäten in der Teilnahmebereitschaft leisten diese Sozialkapitalformen jedoch nicht. Zur Vervollständigung des Forschungspuzzels besteht Bedarf an weiterführenden Untersuchungen, wobei in Ergänzung zu quantitativ empirischen Forschungsstrategien über den Einsatz qualitativer die neue Lebenssituation mit Neugeborenen adäquater erfassenden Methoden nachzudenken ist. Das scheint sinnvoll für die Ergrün- 
dung der in Ein-Elternfamilien vorherrschenden Vorsorgedistanz, welche sich erst durch die Offenlegung subjektiver Relevanzstrukturen besser verstehen lassen dürfte. Möglicherweise stehen der kontinuierlichen Inanspruchnahme des Versorgungssystems schlechte Erfahrungen oder fehlendes Vertrauen in die Einrichtungen des Gesundheitssystems entgegen. Auch wenn in den letzten zwei bis drei Jahrzehnten die Ein-Elternfamilie als neue Familienform quantitativ auf dem Vormarsch war und alleiniges Erziehen in der öffentlichen Wahrnehmung keinen Makel mehr darstellt, können sich Alleinerziehende subjektiv benachteiligt fühlen und Etikettierungen seitens ,erziehungskontrollierender' Akteure im Gesundheits- oder Bildungssystem befürchten. Ein Ungleichheitsbeitrag des Versorgungssystems zur im Durchschnitt niedrigeren Präventionsneigung in Ein-Elternfamilien kann mit den vorliegenden Analysen also nicht ausgeschlossen werden. Analog könnten sich untere Sozial- und Bildungsschichten beim ärztlichen Personal, das mehrheitlich sozial privilegierten Familien entstammt, im Mittel schlechter aufgehoben, schlechter verstanden und in ihrer Erziehungskompetenz vorverurteilt fühlen. Inwiefern solche Prozesse tatsächlich am Werke sind, ist vermutlich zielführender mit offenen statt standardisierten Interview- und Auswertungstechniken zu erfassen. Ein über die qualitative Sozialforschung hergestellter Forschungszugang kann hier ein vielversprechender Ansatz sein. Letzteres gilt sicherlich auch für das bislang geringe Verständnis ethnisch strukturierter Vorsorgedistanzen, die neben sozioökonomischen und sprachlichen Ressourcenbeschränkungen sowie soziokulturell unterschiedlichen Gesundheitskonzepten womöglich Ergebnis ethnischer Diskriminierungsprozesse sind. Mit quantitativen Methoden lassen sich diese empirisch jedoch im Allgemeinen vergleichsweise schwer untersuchen.

\subsection{Bildungsfolgen lebensanfänglicher Gesundheitsungleichheiten}

Einen inhaltlichen Bogen um die ersten zwei Forschungsprobleme spannte die in Kapitel 7 untersuchte dritte Fragestellung. Auf dem Prüfstand stand der Beitrag schwangerschaftsbezogener und postnataler Faktoren zur Erklärung herkunftsabhängiger Bildungsungleichheiten.

Unter Rückgriff auf ressourcen- und expositionstheoretische Überlegungen wurde vermutet, dass zum Lebensbeginn bestehende Entwicklungsdifferenzen zwischen Neugeborenen aus verschiedenen sozialen und ethnischen Gruppen im Vorschulalter kumulieren und schließlich in geringeren Bildungschancen münden. Da bislang wenig gesichertes Wissen zu den mittelund langfristigen Bildungsauswirkungen entwicklungsbezogener Startnachteile vorliegt, empfahl es sich in methodischer Hinsicht explorativ vorzugehen. Für die Analysen bedeutete dies, eine Reihe unterschiedlicher schulischer Erfolgskriterien heranzuziehen, darunter das Ergebnis der Einschulungsuntersuchung, die Grundschulnoten in den übergangsrelevanten Hauptfächern Deutsch und Mathematik sowie ein Maß für die Beteiligung verschiedener sozialer und ethnischer Gruppen in unterschiedlich anspruchsvolle Schulformen der Sekundarstufe. Basis für die Auswertung waren die KIGGS-Daten.

Die deskriptive Untersuchung ergab, dass neben Kindern aus unteren Sozialschichten auch Kinder aus Spätaussiedler- und türkischstämmigen Familien eher zu früh geboren werden. Darüber hinaus ließen sich für türkischstämmige Neugeborene im Durchschnitt häufiger ein 
niedriges Geburtsgewicht sowie tendenziell, auf dem 10 Prozent-Signifikanzniveau, postnatale Gesundheitsprobleme feststellen. Demgegenüber weicht das mittlere Geburtsgewicht von Neugeborenen aus Spätaussiedlerfamilien statistisch nicht bedeutsam vom Gewicht der Vergleichsgruppe ohne Migrationshintergrund ab. In den ersten vier Wochen nach der Geburt schneiden Kinder aus Spätaussiedlerfamilien von allen betrachteten Gruppen gesundheitlich sogar am besten ab.

Wie erfolgreich sind nun Kinder aus unteren Sozialschichten und Migrantenkinder im Bildungssystem? Spiegelbildlich zur Rangreihe in der Frühgeburtsdimension ordnen sich die betrachteten ethnischen Herkunftsgruppen ungleich in der Dimension Bildung an. So erzielen Kinder mit Migrationshintergrund im Mittel schlechtere Noten und positionieren sich im Vergleich zu Kindern aus Nicht-Migrantenfamilien in der Sekundarstufe seltener in einem weiterführenden Schultyp. Im direkten Vergleich schneiden Spätaussiedlerkinder gegenüber türkischstämmigen Kindern schulisch besser ab, was in der Forschungsliteratur gut dokumentiert ist (siehe Gresch und Becker 2010). Letzteres gilt analog für die Chancen auf eine Regeleinschulung. Während sich für türkischstämmige Einschülerinnen und Einschüler ein signifikant erhöhtes Risiko der Rückstellung vom Schulbesuch beobachten lässt, unterscheiden sich Spätaussiedlerkinder diesbezüglich nicht von der Referenzgruppe ohne Migrationsgeschichte. Welche Erkenntnisse hält die Datenauswertung nun für die zentrale Frage nach der lebensgeschichtlich frühen Verursachung herkunftsbezogener Bildungsungleichheiten bereit?

Die multivariaten Analysen ergeben, dass schwangerschaftsbezogene Faktoren (Rauchen), Komplikationen (Frühgeburt), die Säuglingsgesundheit in den ersten vier Lebenswochen sowie elterliche, nach der Geburt getätigte Investitionen in die Entwicklung (regelmäßige Inanspruchnahme der U-Untersuchungen, frühe Einbindung in die formelle Kinderbetreuung) die beobachteten schulischen Erfolgsunterschiede zwischen Kindern mit und ohne ausländische Wurzeln nur partiell vermitteln. Hauptverantwortlich für die ethnisch strukturierten Notenund Positionierungsmuster ist primär der soziale Hintergrund. Schwangerschaftsbezogene Faktoren spielen mit Ausnahme des Rauchverhaltens nur eine Nebenrolle für die Schulleistungen und die Bildungspositionierung. Für die beobachteten ethnischen Bildungsdifferenzen besitzen diese kaum ein Erklärungspotenzial. Im Gegensatz dazu lassen sich ethnische Unterschiede im Einschulungsergebnis jedoch nahezu vollständig auf schwangerschaftsbezogene und sozioökonomische Merkmale zurückführen; eine gemeinsame Betrachtung beider Merkmalsbündel liefert aber keinen zusätzlichen Erklärungsbeitrag. Des Weiteren zeigt sich empirisch, dass bei Kontrolle sozialer Herkunftsmaße weder der Frühgeborenenstatus noch die Variable zur intrauterinen Exposition zu Rauchschadstoffen bedeutsam an Prädiktionskraft verlieren. Beide Faktoren begünstigen eigenständig den erfolgreichen Eintritt in die Grundschule (vgl. Lynch 2011). In abgeschwächter Form gilt letzteres Ergebnis auch für andere betrachtete Bildungskennziffern, wie für die Deutschnote und die Positionierung in einer weiterführenden Schulform statt in einer Haupt- oder Förderschule, was bisherige Studienbefunde stützt (Wolke und Meyer 2000; Jäkel et al. 2013). Im Vergleich zur Einschulung fallen die Assoziationen entwicklungsbezogener Merkmale (Frühgeburt, postnatale Gesundheit) mit den 
Schulnoten aber sehr gering aus. Insbesondere schwache (Deutschnote) oder statistisch unbedeutsame Notenzusammenhänge (Mathematik) stehen dem Forschungsstand eher entgegen: Anders als mit den Modellen kritischer Entwicklungsperioden oder der Akkumulation humanvermögensbezogener Vor- und Nachteile im Lebenslauf erwartet werden konnte, erweisen sich Nachteile in der individuellen Ausgangslage zum Zeitpunkt der Geburt als nahezu irrelevant für die Schulnoten. Ebenso erweist sich die Familiensprache als wenig bedeutsam für die Bildungschancen. Im Kontrast dazu bestätigt sich die Vermutung, dass elterliches Präventionshandeln prädiktive Kraft für die Vorhersage des Besuchs einer Haupt- oder Förderschule (statt einer weiterführenden Schule) sowie guter Noten im Fach Deutsch besitzt. Allerdings stellt sich ein lückenloser Vorsorgestatus nur tendenziell als bedeutsam für die Einschulungschance oder für die Mathematiknote heraus. Insgesamt unterstreichen die Analysen, dass Prävention nicht nur in puncto Gesundheit sondern auch in puncto Bildungschancen statistisch Relevanz besitzt. Allerdings lässt die Untersuchung offen, worauf die Wirkung des Effekts genau beruht: Ist sie auf die Teilnahme an den Untersuchungen zurückzuführen oder steckt vielmehr ein Problem unbeobachteter Heterogenität dahinter?

Insgesamt kann für die dritte Forschungsfrage das regressionsanalytische Ergebnis festgehalten werden, dass ethnische Bildungsdifferenzen über die Betrachtung schwangerschaftsbezogener und postnataler Bedingungen fast vollständig aufgeklärt werden. Im Gegensatz dazu liefern die Analysen kaum empirisch begründete Hinweise, dass auch soziaschichtspezifische Disparitäten in den Einschulungs-, Leistungs- und Positionierungsergebnissen über die hier betrachteten vor- oder nachgeburtlichen Faktoren vermittelt werden.

Vor diesem Ergebnishintergrund lässt sich in Bezug auf die zwei zentralen Ziele der Forschungsarbeit - die Beschreibung und Erklärung herkunftsspezifischer Differenzen in den Dimensionen Schwangerschaftsergebnis und Neugeborenengesundheit und deren Bedeutung für den späteren Bildungserwerb - zusammenfassend folgendes Fazit ziehen (Abschnitt 8.4).

\subsection{Zusammenfassung}

Im Zentrum der Arbeit standen die Herkunftsfamilie und ihre Bedeutung für die Lebenschancen von Kindern. Vor allem die Gesundheit Neugeborener und in abgeschwächter Form auch der Bildungserfolg wurden als familiäre Herstellungsleistung interpretiert. Das theoretische Primat lag auf dem Elternhaus, was anthropologisch mit dem Verweis auf die Abhängigkeit kindlicher Lebenschancen von materiellen und immateriellen elterlichen Zuwendungen begründet wurde. Weiterhin wurde mit der Theorie sozialer Produktionsfaktoren argumentiert, dass alle Menschen nach physischem Wohlbefinden streben, nicht aber alle im Besitz zielführender Mittel sind. Übertragen auf die Kindesgesundheit wünschen sich alle Eltern Gesundheit für ihr(e) Kind(er), ressourcenbedingt können aber nicht alle ein rundum gesundes Aufwachsen ermöglichen. Die in der Arbeit nachgewiesene Abhängigkeit der Schwangerschaftsdauer vom Elternhaus und dessen sozialen Ressourcenpool untermauert dies empirisch und bestätigt die Ergebnisse früherer Studien (Behrman und Butler 2007; Mielck 2005). 
Analog bekräftigen die hier ermittelten Ungleichheitsbefunde den Stand der empirischen Bildungsforschung: So wirkt die Herkunftsfamilie auf die Schulfähigkeit zum Zeitpunkt der Einschulung ein und strukturiert Grundschulleistungen und die Bildungspositionierung.

Schulerfolg hängt dabei signifikant vom elterlichen Gesundheitshandeln $\mathrm{ab}$, ein Befund mit relativem Neuheitsstatus in der Literatur: Je kontinuierlicher die Inanspruchnahme an den UUntersuchungen, desto besser fallen die Noten in den Fächern Deutsch und Mathematik aus. Des Weiteren untermauern die Befunde zum Bildungseinfluss der gesundheitlichen Lebensführung (werdender) Mütter die von der Sozialepidemiologie vorgeschlagenen Modelle der Schwangerschaft als kritischen Entwicklungsperiode: Kinder, die im Mutterleib Rauchschadstoffen ausgesetzt waren, zeigen je nach Dosis der Exposition, entsprechend mehr oder weniger ausgeprägte Leistungsnachteile im Deutsch- und Mathematikunterricht.

Es bestätigt sich somit die zentrale Vorstellung vom Elternhaus als vor- wie nachgeburtlicher Risikofaktor für den Erwerb humanen Vermögens (Bradley und Corwyn 2002). Im Folgenden sollen nun zusammenfassend die zwei im Theorieteil abgeleiteten Arbeitshypothesen (Abschnitt 2.5) hinsichtlich ihrer empirischen Bewährung diskutiert werden. Insgesamt lassen sich diese mit wenigen Ausnahmen bestätigen. Die Ergebnisse der Prüfung sind überblicksartig in den Tabellen 8.1, für Hypothese 1, und 8.2, für die Hypothese 2, dargestellt.

Grundlegend bestätigt sich die Annahme einer sozialen und ethnischen Ungleichverteilung lebensanfänglicher Gesundheitschancen (Hypothese 1a) für den Indikator einer zu kurzen Dauer der Schwangerschaft (Frühgeburt). Dies gilt mit Einschränkung auch für das Geburtsgewicht, wobei entgegen der Erwartung Spätaussiedlerkinder tendenziell schwerer sind als Kinder ohne Migrationshintergrund. Neugeborene Spätaussiedlerkinder schneiden unter allen betrachteten Herkunftsgruppen in den ersten Lebenswochen gesundheitlich sogar am besten $\mathrm{ab}$, während türkischstämmige Neugeborene in der Tendenz eher Gesundheitsprobleme nach der Geburt haben (für einen ähnlichen Befund siehe David und Pachaly 2010).

Ebenso wird die Hypothese 1b bestätigt: Der Hauptanteil der Frühgeburtsvarianz wird über schwangerschaftsbezogene Ungleichheitsbedingungen, das heißt über sozioökonomische Ressourcen und die gesundheitliche Lebensführung (Rauchverhalten) erklärt. Für die Aufklärung ethnisch ungleicher Frühgeburtsprävalenzen spielen medizinische Risikobedingungen (mütterliche Konstitution) hingegen nur eine marginale (Neben-)Rolle. Umgekehrt erweisen sich sozialmedizinische Faktoren für die Erklärung ethnischer Geburtsgewichtsunterschiede von größerer Bedeutung, insbesondere für die Erklärung des im Durchschnitt geringeren Geburtsgewichts türkischstämmiger Neugeborener. Für Spätaussiedlerkinder lassen sich nach Kontrolle sozialmedizinischer Faktoren in der Tendenz (Ergebnisse nicht signifikant) sogar durchschnittliche Gewichtsvorteile gegenüber Kindern aus Nicht-Migrantenfamilien feststellen. Unter den verhaltensbedingten Risiken für nachteilige Schwangerschaftsergebnisse besitzt das Rauchen während der Schwangerschaft, nicht aber Alkoholkonsum Relevanz für das Zustandekommen individueller Nachteile in der entwicklungsbezogenen Ausgangslage zur Geburt. 
Tabelle 8.1: Ergebnisse zur Prüfung von Hypothese 1

\begin{tabular}{|c|c|c|c|c|c|c|c|c|c|}
\hline Hypothese 1 & \multicolumn{3}{|c|}{ Soziale Herkunft ${ }^{1}$} & \multicolumn{6}{|c|}{ Ethnische Herkunft ${ }^{2}$} \\
\hline $\begin{array}{l}\text { Hla: Es gibt eine soziale und ethnische Struktu- } \\
\text { rierung der Gesundheitschancen zum Lebensbe- }\end{array}$ & \multicolumn{3}{|c|}{$\checkmark$} & \multicolumn{6}{|c|}{$(\checkmark)$} \\
\hline \multirow[b]{2}{*}{ Gesundheitsindikatoren } & \multicolumn{3}{|c|}{ Bildung } & \multicolumn{3}{|c|}{ Türkisch } & \multicolumn{3}{|c|}{ Spätaussiedler } \\
\hline & & & & & \\
\hline Frühgeburt (F) & \multicolumn{3}{|c|}{$\checkmark$} & \multicolumn{3}{|c|}{$\checkmark$} & \multicolumn{3}{|c|}{$\checkmark$} \\
\hline Geburtsgewicht (GG) & \multicolumn{3}{|c|}{$\%$} & \multicolumn{3}{|c|}{$(\checkmark)$} & \multicolumn{3}{|c|}{$x$} \\
\hline Postnatale Gesundheit (PG) & \multicolumn{3}{|c|}{.} & \multicolumn{3}{|c|}{$x$} & \multicolumn{3}{|c|}{ 区 } \\
\hline \multirow[t]{3}{*}{$\begin{array}{l}\text { H1b: Ein Teil der Varianz in der Säuglingsge- } \\
\text { sundheit nach sozialen und ethnischen Merkma- } \\
\text { len geht auf schwangerschaftsbezogene Erklä- } \\
\text { rungsgrößen zurück. }\end{array}$} & \multicolumn{3}{|c|}{$\begin{array}{l}\mathrm{FG}(\checkmark) \\
\mathrm{GG} . / \\
\mathrm{PG} . /\end{array}$} & \multicolumn{6}{|c|}{$\begin{array}{l}\text { FG } \checkmark \\
\text { GG } \checkmark \\
\text { PG } \times\end{array}$} \\
\hline & \multicolumn{3}{|c|}{ Bildung } & \multicolumn{3}{|c|}{ Türkisch } & \multicolumn{3}{|c|}{ Spätaussiedler } \\
\hline & $\mathrm{F}$ & GG & PG & $\mathrm{F}$ & GG & PG & $\mathrm{F}$ & GG & $\mathrm{PG}$ \\
\hline \multicolumn{10}{|l|}{$\begin{array}{l}\text { Partielle Mediation durch schwangerschaftsbe- } \\
\text { zogene Faktoren: }\end{array}$} \\
\hline Ressourcen (bzw. Schicht) + Lebensführung & $\checkmark$ & $\%$ &. & $\checkmark$ & $\checkmark$ & $(\checkmark)$ & $\checkmark$ & $*$ & $x$ \\
\hline Medizinische Risiken (Konstitution) & $x$ &. &. & $(\checkmark)$ & $\checkmark$ & $x$ & $x$ & $*$ & $x$ \\
\hline $\begin{array}{l}\text { Vollständige Mediation lebensanfänglicher } \\
\text { Nachteile bei gemeinsamer Berücksichtigung } \\
\text { aller Faktoren (siehe Endmodelle in Kapitel) }\end{array}$ & $(\checkmark)$ &. & $\%$ & $\checkmark$ & $\checkmark$ & $x$ & $\checkmark$ & * & $x$ \\
\hline
\end{tabular}

Symbolik: $\checkmark=$ H. bestätigt, $(\checkmark)=$ H. teils bestätigt, $\mathbb{\bigotimes}=$ H. nicht bestätigt (umgekehrter Effekt), $\mathbf{x}=$ H. nicht bestätigt, ./. = nicht (regressionsanalytisch im Detail) untersucht; $*=$ empirisch keine bedeutsamen Ausgangsdifferenzen beobachtet.

Anmerkungen: ${ }^{1}$ Ergebnisse auf Basis der SOEP-Daten (für Herkunftsmerkmal Bildung, siehe Kapitel 5); ${ }^{2}$ Ergebnisse auf Basis der KiGGS-Daten (siehe Kapitel 8).

Wie Tabelle 8.2 zeigt, lässt sich die zweite Hauptannahme mit Einschränkungen bestätigen. Zum einen finden sich in den Analysen statistisch (hoch-)bedeutsame Assoziationen zwischen der sozialen und ethnischen Herkunft und den vier Bildungs- und Erfolgsindikatoren (H2a), mit einer Ausnahme: Für das Kriterium Rückstellung vom Schulbesuch wird für die Spätaussiedlergruppe kein Chancennachteil gegenüber der Referenzgruppe ohne Migrationshintergrund beobachtet. Zum anderen lässt sich ein Teil der gruppenspezifischen Bildungs- und Erfolgsvarianzen über schwangerschaftsbezogene Faktoren aufklären, dieser fällt aber insgesamt eher gering aus. Hypothese $2 \mathrm{~b}$ findet empirisch also keine uneingeschränkte Unterstützung, nicht zuletzt deshalb, weil (mit Ausnahme der sozialen Herkunft) nachgeburtliche Bildungsfaktoren, darunter sprachkulturelle und frühbildungsbezogene Faktoren (Kindertagesbetreuung), kaum Erklärungskraft besitzen. Nichtsdestotrotz leistet die gemeinsame Berücksichtigung aller erklärenden Variablen eine vollständige Aufklärung der bei Kindern mit familiärer Migrationsbiographie beobachteten Chancennachteile im deutschen Bildungssystem. 
Tabelle 8.2: Ergebnisse zur Prüfung von Hypothese 2

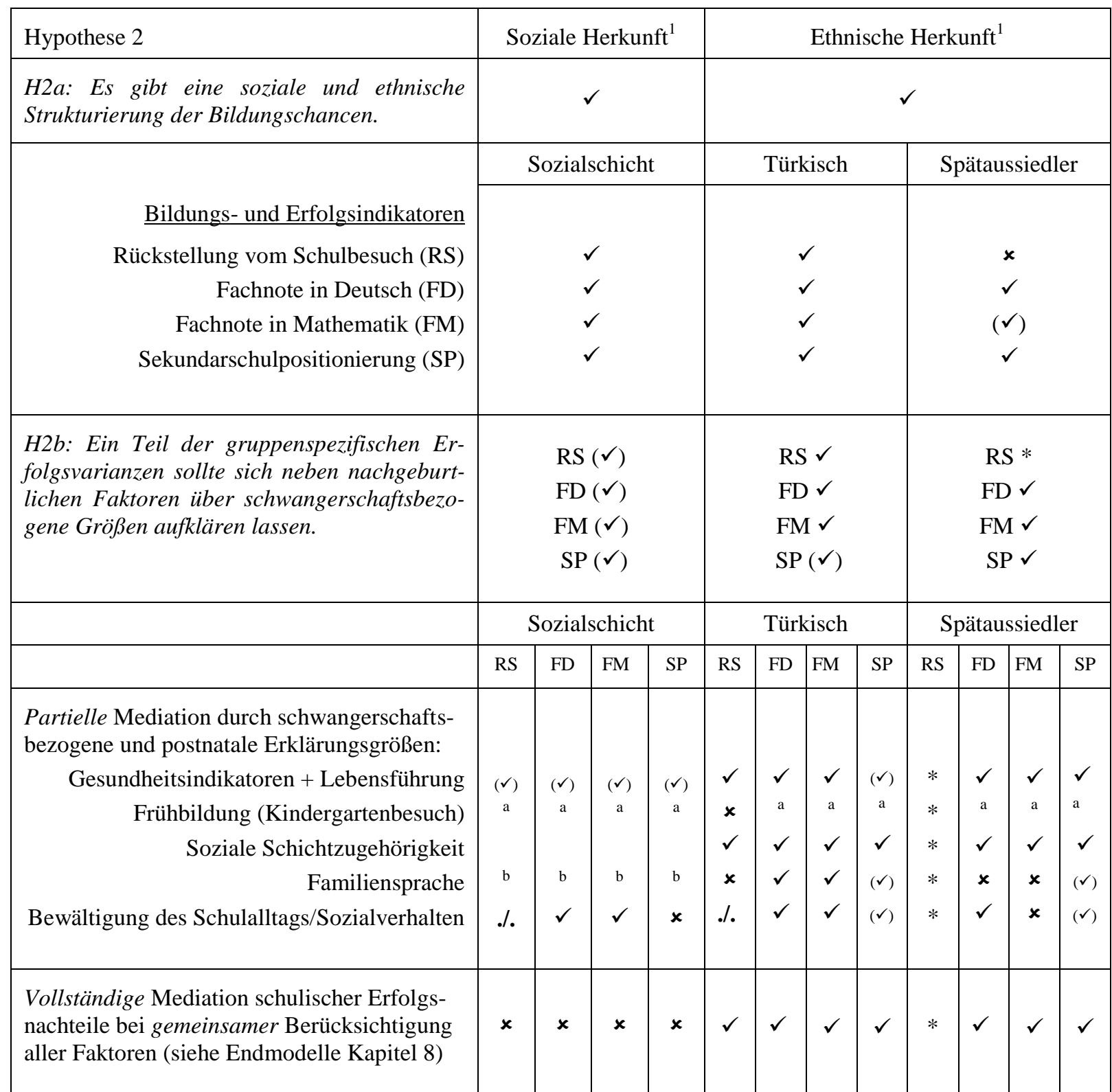

Symbolik: $\checkmark=H$. bestätigt, $(\checkmark)=H$. teils bestätigt, $\bigotimes=H$. nicht bestätigt (umgekehrter Effekt), $x=H$. nicht bestätigt, ./. = nicht (regressionsanalytisch im Detail) untersucht; * = empirisch keine bedeutsamen Ausgangsdifferenzen beobachtet. ${ }^{a}$ In den Analysen zu den Grundschulnoten und der Bildungspositionierung in der Sekundarstufe wurde der Kindergartenbesuch aus Platzgründen zusammen mit den Indikatoren zur Lebensführung in die Regressionsmodelle eingeführt; ${ }^{b}$ Keine Hypothese zur Vermittlung schichtspezifischer Effekte formuliert.

Anmerkungen: ${ }^{1}$ Ergebnisse auf Basis der KiGGS-Daten (siehe Kapitel 8).

Mit Blick auf den theoretischen Rahmen kann festgehalten werden, dass der ressourcen- und investitionstheoretische Überbau empirisch gut greift. Die Untersuchungsbefunde legen dabei nicht nur einen zweistufigen Statusvererbungsprozess (wie bei Boudon) sondern einen $d r e i$ stufigen Prozess nahe. Nach Boudon (1974) durchlaufen Kinder verschiedener sozialer Gruppen (Klassen) mit unterschiedlichem Erfolg das Bildungssystem, was sich in einer sozial strukturierten Bildungsbeteiligung ausdrückt und in Verteilungsungleichheiten im Erwerb prestigereicher Bildungsabschlüsse mündet. Der zweite hier nicht untersuchte Prozess bezieht 
sich auf die Verwertbarkeit der auf dem Arbeitsmarkt unterschiedlich nachgefragten Bildungstitel, wobei höhere zur Hochschule führende Zertifikate Chancenvorteile bei der sozialen Statuspositionierung (,inequality of social opportunity“: ISO) mit sich bringen und damit die Berechtigung zur Platzierung in eine obere soziale Klasse.

Wie die vorliegende Arbeit und ihre zentralen empirischen Befunde zeigen, lässt sich mit der entwicklungsbezogenen Ausgangslage zum Zeitpunkt der Geburt ein weiterer Prozessabschnitt modellieren. Die Geburt wurde im Theorieteil als Transition von der intrauterinen Lebensumwelt (Bauch der Mutter) in die extrauterine Lebensumwelt (im Kleinen: die Familie, im Großen: die Gesellschaft) interpretiert. Der Übergang selbst ist dabei Endpunkt eines kritischen Entwicklungsprozesses und wird von Neugeborenen je nach sozialer Klassen- und ethnischer Zugehörigkeit unterschiedlich bewältigt. Das ist die gesundheitliche Ungleichheit, die terminologisch als IHO bzw. inequality of health opportunity bezeichnet wurde.

Dabei wurde im konzeptionellen Teil bezüglich der Vererbung von Statusvorteilen im Generationenverlauf dargelegt, dass das Problem gesundheitlicher Ungleichheit zum Lebensbeginn dem Problem ungleicher Bildungsbeteiligung vorgeschaltet ist. Hierfür müssten sich Bildungsungleichheiten aus schwangerschaftsbezogenen Ungleichheiten ergeben. Wie die in Kapitel 7 präsentierten Untersuchungen zeigen, ist dies insbesondere für das Einschulungsergebnis und in abgeschwächter Form für die Bildungspositionierung in der Sekundarstufe der Fall: Im Vergleich zu Termingeborenen haben Frühgeborene (vermutlich aufgrund einer eingeschränkten Schulfähigkeit) ein höheres Risiko ein Jahr vom Besuch der Grundschule zurückgestellt zu werden. Auch haben sie in der Sekundarstufe eine höhere Wahrscheinlichkeit eine Haupt- oder Förderschule zu besuchen. Ob zuletztgenanntes Muster über primäre oder sekundäre Effekte der familiären Herkunft und/oder Lehrereinflüsse erzeugt werden, kann mit den verwendeten Daten nicht beantwortet werden. Ungeachtet dieser Einschränkung zeigt sich jedoch, dass eine erfolgte Rückstellung vom Schulbesuch statistisch mit der Bildungspositionierung assoziiert ist. Eine weitere sich in den konzeptionellen Rahmen einfügende Beobachtung ist die stabile Assoziation zwischen allen betrachteten Bildungskennziffern mit der Variablen zur Exposition zu Rauchschadstoffen während der Schwangerschaft. Diese Befunde können als Hinweise darauf verstanden werden, dass sich der Statusvererbungsprozesses zum Teil über vorgeburtlich wirksam werdende Prozessketten vollzieht, etwa über folgende: Rauchexposition - Gesundheitsnachteile zur Geburt - vorschulische Kompetenzbeeinträchtigungen - geringere Bildungschancen. Für eine abschließende Bewertung schwangerschaftsbezogener Ungleichheitsmechanismen sind aber weitere Forschungsanstrengungen vonnöten.

\subsection{Fazit}

Im Bereich Bildung prägte in den 60er Jahren des vergangenen Jahrhunderts das „katholische Arbeitermädchen vom Lande“ das Ungleichheitsbild in Deutschland (Dahrendorf 1965; Peisert 1967). In den 90er Jahren hatte sich diese symbolische Kunstfigur statistisch nahezu aufgelöst, ihr Platz unter den am stärksten benachteiligten Gruppen im deutschen Bildungssystem blieb aber nicht lange leer und wurde von einer in schulischer Hinsicht ebenfalls relativ 
wenig erfolgreichen Kunstfigur eingenommen - dem ,türkischen Arbeitersohn im Großstadtquartier“ (Geißler 2005; Baumert und Schümer 2001; R. Becker 2011). Beide Bildungsgestalten, die ältere wie die neuere, teilen die Gemeinsamkeit, in ein sozial unterprivilegiertes Elternhaus geboren zu sein, ein für den vorschulischen Kompetenzerwerb und späteren Bildungswettbewerb in der Regel ungünstiges Vorzeichen.

Die vorliegenden Befunde erlauben dieser aktuellen Kunstfigur eine gesundheitsbezogene Facette hinzuzufügen. So finden sich in den durchgeführten Analysen neben eindeutigen Belegen für schulische Nachteile bei Kindern aus unteren sozialen Schichten und Migrantengruppen auch in gesundheitlicher Hinsicht Benachteiligungen, und zwar im Neugeborenenalter. Dabei hängen schwangerschaftsbezogene Ungleichheitsbedingungen bedeutsam mit dem späteren Bildungs(miss)erfolg zusammen. Die empirische Erhärtung der Frühverursachungshypothese in Form der hochsignifikanten Abhängigkeit des Einschulungsergebnisses von der Schwangerschaftsdauer sowie der stark negativen Wirkung der gesundheitsriskanten Lebensführung während der Schwangerschaft (Rauchen) auf den Bildungserfolg wirft in demokratischer (Chancengleichheit) wie wirtschaftlicher Hinsicht (Stichworte: Bildungsausschöpfung; Kompensationskosten) soziale Fragen auf. Daneben kann ein in entwicklungsbezogener Hinsicht nachteiliger Start ins Leben aber nicht nur für das Gemeinwesen, sondern für frühgeborene Kinder selbst und ihre Familien negative Konsequenzen nach sich ziehen. Gerade Eltern, welche sich um das Überleben und künftigen Lebenschancen der Frühchen sorgen, müssen mit hohen psychischen Belastungen zurechtkommen (Kirschner et al. 2009; Goldberg und Divitto 2002) - eine Aufgabe, die vermutlich insbesondere ressourcenärmere Familien schwerer bewältigen können. Wenig Optimismus verbreitet in diesem Zusammenhang wachsende Kinderarmut (Richter und Hurrelmann 2006), aus welcher sich für in sozial weniger privilegierte Elternhäuser geborene Frühchen ein mehrfaches Bildungsrisiko ableitet.

Mit Blick auf den Gesundheits- und Bildungskarrieren lenkenden Einfluss der verhaltensbezogenen Exposition zu Umweltgiften (Rauchen) empfiehlt es sich, dass zukünftige Studien sich verstärkt dem „Handeln selbst“ (Berger 1963: 290) zuwenden und danach fragen, warum Schwangere aus unteren Sozialschichten im Mittel seltener auf das Rauchen während der Schwangerschaft verzichten. Eine Beantwortung dieser Frage besitzt hohe gesundheits- und bildungspolitische Relevanz. So hinkt, wie die vorliegenden Untersuchungsergebnisse zeigen, ein bedeutsamer Teil der während der intrauterinen Entwicklung Rauchschadstoffen ausgesetzten Schülerschaft hinsichtlich der Schulfähigkeit zum Zeitpunkt der Einschulung sowie dem Leistungsstand in den Fächern Deutsch und Mathematik hinter ihrer Vergleichsgruppe (der diesem Risiko nicht ausgesetzten Schülerschaft) hinterher. Vor diesem Hintergrund überrascht auch nicht, dass das (mütterliche) Rauchverhalten während der Schwangerschaft nicht folgenlos bleibt für die individuellen Positionierungschancen in der Sekundarstufe und somit auch nicht für den letztlich erworbenen Bildungsabschluss. Rauchen ist also für den Erwerb humanen Vermögens, sei es Gesundheit oder Bildung, ein weichenstellender - wie vermeidbarer (!) - Risikofaktor. Statistisch hochbedeutsame Zusammenhänge zwischen Rauchexposition und den verwendeten Schulerfolgsmaßen legen irreversible kognitive Beeinträchtigungen 
nahe, welche sich im Erwachsenenalter in geringeren Arbeitsmarkt- und Einkommenschancen und damit auch in einer geringeren Lebenserwartung niederschlagen können.

Nichtsdestotrotz stellen diese Befunde, wie auch andere soziale Ungleichheitsstrukturen, keine unumstößlichen Naturgesetze dar. Im Gegenteil: Soziale Ungleichheitsphänomene sind in der Regel menschengemacht und daher veränderbar (Kreckel 1992: 13). Allerdings entziehen sich soziale Unterschiede in den Lebens- und Handlungsbedingungen typischerweise der kurzfristigen Einflussnahme durch den Menschen; Ungleichheiten werden „häufig als unabänderliches Schicksal hingenommen“ (Kreckel 1992: 13; Solga al. 2009: 15) und darüber legitimiert und verfestigt (Berger 2004: 355). Pessimistisch betrachtet, trügt die Hoffnung der Sozialpolitik, mit Bildung(-sforschung) allein gesellschaftliche Probleme lösen zu können (Solga und Becker 2012: 10). Dafür fördert dieser Glauben, so Solga und Becker (2012: 11), das „Interesse an Bildungsforschung“. Von ähnlich wenig Optimismus getragen scheint die Diskussion innerhalb der gesundheitssoziologischen Forschung.

Die Politik versucht aber Einfluss auf die Lebenschancen von Kindern zu nehmen. Eine im Sinne der Sozialinvestition unlängst abgewickelte Gesetzesreform war die Ablösung des Prinzips der freiwilligen Teilnahme an den U-Untersuchungen durch die Einführung eines verbindlichen Einlade- und Meldewesens. Diese Reform ist kritisch zu sehen, da vor dem Grundgesetz (Art. 6, Absatz 2) die primäre Verantwortung für die Pflege und Erziehung der Kinder bei den Eltern liegt. Die Einflussnahme von oben kann zwar einem gesamtgesellschaftlichen Interesse dienlich sein, dies allerdings auf einem schmalen Grat zwischen Hilfsangebot und Kontrolle. Nichtsdestotrotz verpflichtet das demokratische Selbstverständnis moderne Gesellschaften, die Hoffnung nicht aufzugeben, mittels sozialpolitisch intervenierender Maßnahmen sozial ungleiche Lebenschancen modifizieren (abschwächen) zu können.

In diesem Kontext dominiert im gesundheits- und bildungspolitischen Diskurs wie in der gesundheits- und bildungssoziologischen Forschung die Frage nach dem Kompensationspotential institutioneller Einrichtungen der Prävention oder frühkindlichen Bildung und Betreuung (Spieß 2013; Anders 2013). Wie die Ergebnisse aus Kapitel 7 zeigen, hängen Prävention und Bildung zusammen: Kinder, die nur unregelmäßig an den U-Untersuchungen teilnehmen, haben eine um das 10\%-Signifikanzniveau schwankende höhere Wahrscheinlichkeit vom Schulbesuch zurückstellt zu werden. Wesentlich prägnanter fallen die Assoziationen zwischen Präventionsneigung auf der einen und Grundschulnoten in Mathematik und Deutsch sowie die Bildungspositionierung auf der anderen Seite aus. Bemerkenswert ist dabei, dass die positiven Präventionseffekte für den Notenerfolg (nicht aber die für die Positionierung in einem weiterführenden Sekundarschulzweig) nach statistischer Berücksichtigung der individuellen Bewältigung des Schulalltags und dem Sozialverhalten zurückgehen. In anderen Worten: Der Einfluss schul- und lernrelevanter Größen, welche explizit im Fokus der U-Untersuchungen stehen, auf die Notengüte wird partiell über das elterliche Präventionsverhalten vermittelt. Insofern kann im Anschluss an die bisherige Forschung (Jungbauer-Gans 2006) resümiert werden, dass eine geringe elterliche Präventionsneigung am Zustandekommen des schlechteren schulischen Abschneidens beteiligt ist. Unklar bleibt, welcher Mechanismus hier greift: Sind es 
frühzeitig identifizierte und anschließend kompensierte Entwicklungsbeeinträchtigungen oder die in die U-Untersuchungen integrierte Beratungskomponente, die elterliche Erziehungs- und Gesundheitskompetenzen fördern sollen? Oder reflektieren die vom regelmäßigen U-Besuch ausgehenden positiven Bildungseffekte unbeobachtete familiäre Umweltbedingungen?

Hinsichtlich der Wirkung institutioneller Kontexte, wie sie für die formelle Kinderbetreuung diskutiert werden, erlauben die Ergebnisse der vorliegenden Arbeit auch eine - die in sie gesetzten Bildungshoffnungen nicht enttäuschende - Einschätzung. So finden sich für die Erfolgskriterien Fachnoten und Bildungspositionierung (nicht aber für die Einschulung) signifikante Zusammenhänge mit dem Kindergarteneintrittsalter: In der Regel zahlen sich ein Betreuungsbeginn vor Vollendung des dritten Lebensjahres in Form besserer Grundschulnoten und höheren Chancen auf die Einmündung in einen weiterführenden Sekundarschultyp aus. Allerdings sind diese Befunde im Hinblick auf die Ungleichheitsperspektive nur eingeschränkt positiv zu bewerten. Werden in den multiplen Regressionsmodellen familiäre Herkunftsmerkmale (Schichtzugehörigkeit, Migrationshintergrund) berücksichtigt, verliert der Einfluss des Alters des Kindes bei Betreuungsbeginn an Stärke und ist in einigen Modellen nicht mehr auf dem $10 \%$-Signifikanzniveau nachweisbar. Diese Beobachtung ist insofern nicht überraschend, als dass die frühe Nutzung von Kindertageseinrichtungen sozial selektiv erfolgt, also obere soziale Klassen sowie Familien aus der Mehrheitsgesellschaft zu einem größeren Anteil ihre Kinder bereits vor dem dritten Geburtstag zur Betreuung in eine Kindertageseinrichtung geben, weil deren Mütter beispielsweise im Mittel stärkere Präferenzen für eine baldige Wiederaufnahme der Erwerbstätigkeit, für eine familienergänzende Kinderbetreuung und Frühbildung aufweisen oder in finanzieller und wohnortbezogener Hinsicht eine auf pro-Kindertagesbetreuung ausgerichtete Opportunitätsstruktur vorfinden. Andere Studien (Becker und Lauterbach 2008) weisen in eine ähnliche Richtung, das heißt ein (Groß-)Teil der positiven formellen Betreuungseffekte geht auf das Konto der sozialen Herkunft bzw. unterliegt einem Selektionseffekt. Insofern stützen die hier gefundenen Befunde den Forschungsstand für Deutschland, demgemäß Kindertagesstätten zwar eine bedeutsame, relativ zum Einfluss der Herkunftsfamilie aber eher nebengeordnete Rolle für den Bildungserfolg spielen (Anders 2013). ${ }^{71}$ Allerdings erlauben die hier vorgestellten Befunde datenbedingt keine Aussagen zur Qualität dieser Einrichtungen.

Schließlich sei mit Blick auf den Schuleingang kurz auf die Möglichkeit einer diskriminierenden Wirkung der schulärztlichen Eingangsuntersuchung (Gate-Keeping: Gomolla und Radtke 2002) eingegangen. Zwar deuten die Befunde auf einen schulischen Nachteil für die ehemals vom Schulbesuch zurückgestellten Kinder hin, der an im Mittel schlechteren Noten in Deutsch und Mathematik festgemacht werden kann, allerdings lassen sich in den schrittweise aufgebauten Regressionsmodellen, in denen das Merkmal Zurückstellung einzeln in die Regression eingeführt wurde, keine Hinweise auf Diskriminierungseffekte beobachten. So verändern sich die mit einem Migrationshintergrund assoziierten Bildungsnachteile in Stärke

\footnotetext{
${ }^{71}$ Anders (2013) beschränkt dieses Resümee auf Kindertagesstätten von hoher Struktur- und Prozessqualität.
} 
und Richtung nicht nach Aufnahme der Einschulungserfahrung in das Regressionsmodell. Für die Aufklärung ethnischer Leistungs- und Positionsdisparitäten bedeutet also die Tatsache, ob ein Kind vom Grundschulbesuch zurückgestellt wurde oder nicht keinen Erklärungsgewinn.

Insgesamt liefert die Forschungsarbeit Hinweise auf schwangerschaftsbezogene Ursachen für gesundheitliche und bildungsbezogene Ungleichheiten, die aber aufgrund von Fallzahlproblemen und methodischen Einschränkungen bei der Operationalisierung zentraler theoretischer Konstrukte repliziert werden sollten (Becker und Stolberg 2013; Stolberg und Becker 2015). Mit den Lebensverlaufsdaten des Nationalen Bildungspanels (NEPS) kann dieses Unterfangen auf eine vielversprechende Grundlage gestellt werden, was die Diskussion um die pränatale Genese ungleicher Lebenschancen und die Frage, ob soziale Chancengleichheit im Bildungssystem eine Utopie bleiben muss, zukünftig um belastbarere Evidenzen bereichert. 


\section{Literatur}

Abel, Thomas. 2007. Cultural capital in health promotion. In Health and modernity. The role of theory in health promotion, Hrsg. David V. Mcqueen und Ilona Kickbusch, 43-73. Heidelberg: Springer.

Abel, Thomas. 2008. Cultural capital and social inequality in health. Journal of Epidemiology and Community Health 62:e13-e18.

Abel, Thomas, Andrea Abraham, und Kathrin Sommerhalder. 2009. Kulturelles Kapital, kollektive Lebensstile und die soziale Reproduktion gesundheitlicher Ungleichheit. In $\mathrm{Ge}$ sundheitliche Ungleichheit. Grundlagen, Probleme, Perspektiven, Hrsg. Matthias Richter und Klaus Hurrelmann, 195-208. Wiesbaden: VS Verlag für Sozialwissenschaften.

Alba, Richard D., Johann Handl, und Walter Müller. 1994. Ethnische Ungleichheiten im deutschen Bildungssystem. Kölner Zeitschrift für Soziologie und Sozialpsychologie 46:209237.

Alderliesten, Marianne, Tanja Vrijkotte, Marieke van der Wal, und Gouke Bonsel. 2007. Late start of antenatal care among ethnic minorities in a large cohort of pregnant women. BJOG, An International Journal of Obstetrics and Gynaecology 114:1232-1239.

Almond, Douglas, und Bhashkar Mazumder. 2013. Fetal origins and parental responses. Annual Review of Economics 5:37-56.

Ancél, Pierre-Yves, Marie-Josephe Saurel-Cubizolles, Gian Carlo Di Renzo, Emile Papiernik, und Gérard Bréart. 1999a. Very and moderate preterm births: Are the risk factors different? British Journal of Obstetrics and Gynaecology 106:1162-1170.

Ancél, Pierre-Yves, Marie-Josèphe Saurel-Cubizolles, Gian Carlo Di Renzo, Emile Papiernik, Gérard Bréart und Europop Group. 1999b. Social differences of very preterm birth in Europe: Interaction with obstetric history. American Journal of Epidemiology 149:908-915.

Anders, Yvonne. 2013. Stichwort: Auswirkungen frühkindlicher institutioneller Betreuung und Bildung. Zeitschrift für Erziehungswissenschaften 16:237-275.

Andersen, Ronald M. 1995. Revisiting the behavioral model and access to medical care: Does it matter? Journal of Health and Social Behavior 36:1-10.

Antonovsky, Aaron.1979. Health, Stress and Coping. San Francisco: Jossey-Bass.

Arpi, Elena, und Fabrizio Ferrari. 2013. Preterm birth and behaviour problems in infants and preschool-age children: a review of the recent literature. Developmental Medicine \& Child Neurology 55:788-796.

Auspurg, Katrin, und Thomas Hinz. 2011. Gruppenvergleiche bei Regressionen mit binären abhängigen Variablen - Probleme und Fehleinschätzungen am Beispiel von Bildungschancen im Kohortenverlauf. Zeitschrift für Soziologie 40:62-73.

Autorengruppe Bildungsberichterstattung. 2012. Bildung in Deutschland 2012. Ein indikatorengestützter Bericht mit einer Analyse zur kulturellen Bildung im Lebenslauf. Bielefeld: W. Bertelsmann Verlag. 
Barker, David J.P., Johan G. Eriksson, Tom Forsen, und Clive Osmond. 2002. Fetal origins of adult disease: strength of effects and biological basis. International Journal of Epidemiology 31:1235-1239.

Barnard, Kathryn E., und JoAnne E. Solchany. 2002. Mothering. In Handbook of Parenting. Vol. 3: Being and Becoming a Parent. Second Edition, Hrsg. Marc H. Bornstein, 3-25. Mahwah, NJ, US: Lawrence Erlbaum Associates Publishers.

Baumert, Jürgen, und Gundel Schümer. 2001. Familiäre Lebensverhältnisse, Bildungsbeteiligung und Kompetenzerwerb. In PISA 2000. Basiskompetenzen von Schülerinnen und Schülern im internationalen Vergleich, Hrsg. Deutsches PISA-Konsortium, 323-407. Opladen: Leske + Budrich.

Beck, Stacey, Daniel Wojdyla, Lale Say, Ana Pilar Betran, Mario Merialdi, Jennifer Harris Requejo, Craig Rubens, Ramkumar Menon, und Paul F.A. Van Look. 2010. The worldwide incidence of preterm birth: a systematic review of maternal mortality and morbidity. Bulletin of the World Health Organization 88:31-38.

Becker, Birgit, und Nicole Biedinger. 2006. Ethnische Bildungsungleichheit zu Schulbeginn. Kölner Zeitschrift für Soziologie und Sozialpsychologie 58:660-684.

Becker, Gary S. 1981. A treatise on the family. Cambridge.

Becker, Gary S. 1993. Human Capital. A Theoretical and Empirical Analysis with Special Reference to Education. Chicago: University Press.

Becker, Rolf. 2009. Bildungssoziologie - Was sie ist, was sie will, was sie kann. In Lehrbuch der Bildungssoziologie, Hrsg. Rolf Becker, 9-34. Wiesbaden: VS Verlag für Sozialwissenschaften.

Becker, Rolf. 2011. Lehrbuch der Bildungssoziologie. Wiesbaden: VS Verlag für Sozialwissenschaften.

Becker, Rolf, und Wolfgang Lauterbach. 2008. Bildung als Privileg - Ursachen, Mechanismen, Prozesse und Wirkungen. In Bildung als Privileg, Hrsg. Rolf Becker und Wolfgang Lauterbach, 11-45. Wiesbaden: VS Verlag für Sozialwissenschaften.

Becker, Sten. 2016. Lebensanfängliche Gesundheitsunterschiede und ihre Bildungsfolgen. (Unveröffentlichtes Manuskript).

Becker, Sten, und Karin Kurz. 2011. Social Inequality in Early Childhood Health. Participation in the Preventive Health Care Program for Children. Schmollers Jahrbuch - Journal of Applied Social Science Studies 131:381-394.

Becker, Sten, und Carolyn Stolberg. 2013. Ethnic inequality in preterm birth. Schmollers Jahrbuch - Journal of Applied Social Science Studies 133:299-322.

Beckert-Zieglschmid, Claudia. 2005. „Der Apfel fällt nicht weit vom Stamm?“ Eine Anwendung der Theorie von Pierre Bourdieu auf Lebensstile und Ernährungspraxis Jugendlicher. Norderstedt: Books on Demand.

Behrens, Johann. 2006. Meso-soziologische Ansätze und die Bedeutung gesundheitlicher Unterschiede für die allgemeine Soziologie sozialer Ungleichheit. In Gesundheitliche Un- 
gleichheit. Grundlagen, Probleme, Perspektiven, Hrsg. Matthias Richter und Klaus Hurrelmann, 53-72. Wiesbaden: VS Verlag für Sozialwissenschaften.

Behrman, Richard E., und Adrienne Smith Butler. 2007. Preterm birth. Causes, consequences, and prevention. Washington, D.C: The National Academies.

Benedek, Therese.1959. Parenthood As A Developmental Phase: A Contribution to the Libido Theory. Journal of the American Psychoanalytic Association 7:389-417.

Ben-Shlomo, Yoav, und Diana Kuh. 2002. A life course approach to chronic disease epidemiology: conceptual models, empirical challenges and interdisciplinary perspectives. International Journal of Epidemiology 31:285-293.

van den Berg, Gerrit, Manon van Eijsden, Tanja Vrijkotte, und Reinoud Gemke. 2012. Educational inequalities in perinatal outcomes: The mediating effect of smoking and environmental tobacco exposure. PLoS ONE 7:e37002.

Berger, Claudia, und Beate Schücking. 2011. Welchen Einfluss hat der Migrationshintergrund auf das geburtshilfliche Outcome? Die Hebamme:116-119.

Berger, Johannes. 2004. Über den Ursprung der Ungleichheit unter den Menschen. Zur Vergangenheit und Gegenwart einer soziologischen Schlüsselfrage. Zeitschrift für Soziologie 33:354-374.

Berger, Peter L. 1963. Einladung zur Soziologie. München: DTV.

Bergmann, Karl E., Renate L. Bergmann, Ute Ellert, und Joachim W. Dudenhausen. 2007. Perinatale Einflussfaktoren auf die spätere Gesundheit. Ergebnisse des Kinder- und Jugendgesundheitssurveys (KiGGS). Bundesgesundheitsblatt - Gesundheitsforschung Gesundheitsschutz 50:661-674.

Bergmann, Renate L., und Joachim Wolfram Dudenhausen. 2003. Prädiktion und Prävention der Frühgeburt. Der Gynäkologe 36:391-402.

Bermaoui, Jonas, Carina Marten, Ilona Ostner, Thorben Sembritzki, und Carolyn Stolberg. 2012. Parenting programmes in Germany - an overview. Polchi Working Paper, GeorgAugust University Göttingen.

Bernardi, Fabrizio. 2014. Compensatory Advantage as a Mechanism of Educational Inequality. A Regression Discontinuity Based on Month of Birth. Sociology of Education 87:7488.

Bernthaler, Peter. 2009. Frühstart ins Leben: Risiken und Perspektiven. Pädiatrie \& Pädologie 44:22-23.

Berthold, Barbara. 2008. Einschulungsregelungen und flexible Eingangsstufe. Recherche für den Nationalen Bildungsbericht 2008 im Auftrag des Deutschen Jugendinstituts Stand: Februar 2008. München: Verlag Deutsches Jugendinstitut.

Berufsverband der Kinder- und Jugendärzte e. V. (BVKJ). o.J. http://www.kinderaerzte-imnetz.de/vorsorge/baby-u1-bis-u6/u6-sechste-vorsorge/ (Zugriff: 22.02.2016). 
Bettge, Susanne, Sylke Oberwöhrmann, Matthias Brockstedt, und Christoph Bührer. 2014. Birth weight and special educational needs - results of a population based study in Berlin. Deutsches Ärzteblatt International 111:337-344.

Bettge, Susanne, Sylke Oberwöhrmann, Sabine Hermann, und Gerhard Meinlschmidt. 2007. Kindergesundheit in Berlin. Problemfelder und Handlungsbedarf. Gesundheits- und Sozialpolitik 61:34-48.

Biedinger, Nicole, und Birgit Becker. 2006. Ethnische Bildungsungleichheiten zu Schulbeginn. Kölner Zeitschrift für Soziologie und Sozialpsychologie 58:660-684.

Blau, Peter M., und Otis D. Duncan. 1967. The American occupational structure. New York: Wiley.

Bloomfield, Kim, Ludwig Kraus, und Michael Soyka. 2008. Alkoholkonsum und alkoholbezogene Störungen (Gesundheitsberichtserstattung des Bundes, Heft 40). Berlin: Robert Koch Institut.

Blossfeld, Hans-Peter. 1988. Bildungsverläufe im historischen Wandel. In Bildung, Beruf, Arbeitsmarkt, Hrsg. Hans-Joachiam Bodenhöfer, 259-302. Berlin: Ducker \& Höfer.

Blossfeld, Hans-Peter, Jutta von Maurice, und Thorsten Schneider. 2011. Grundidee, Konzeption und Design des Nationalen Bildungspanels für Deutschland. NEPS Working Paper No. 1. Bamberg: Otto-Friedrich-Universität, Nationales Bildungspanel.

Blumenshine, Philip, Susan Egerter, Colleen J. Barclay, Catherine Cubbin, und Paula A. Braveman. 2010. Socioeconomic disparities in adverse birth outcomes: A systemativ review. American Journal of Preventive Medicine 39:263-272.

Blumenshine, Philip, Susan Egerter, Moreen Libet, und Paula A. Braveman. 2011. Father's education: An independent marker of risk for preterm birth. Maternal Child Health Journal 15:60-67.

Bollini, Paola, Sandro Pampallona, Philippe Wanner, und Bruce Kupelnick. 2009. Pregnancy outcome of migrant women and integration policy: A systematic review of the international literature. Social Science and Medicine 68:452-461.

Bolten, Margarete. 2012. Stressfaktor drohende Frühgeburt. Psychoscope 5:4-7.

Borgetto, Bernhard, und Karl Kälble. 2007. Medizinsoziologie. Sozialer Wandel, Krankheit, Gesundheit und das Gesundheitssystem. Weinheim und München: Juventa Verlag.

Boudon, Raymond. 1974. Education, Opportunity, and Social Inequality. New York: John Wiley \& Sons.

Boudon, Raymond. 1998. Social mechanisms without black boxes. In Social Mechanisms: An Analytical Approach to Social Theory, Hrsg. Peter Hedström und Richard Swedberg, 172-203. Cambridge: Cambridge University Press.

Bourdieu, Pierre. 1973. Kulturelle Reproduktion und soziale Reproduktion. In Grundlagen einer Theorie der symbolischen Gewalt, Hrsg. Pierre Bourdieu, 88-137. Frankfurt am Main: Suhrkamp. 
Bourdieu, Pierre. 1976. Kulturelle Reproduktion und soziale Reproduktion. In Soziale Ungleichheit und Prozesse Sozialer Schichtung, Hrsg. Karl H. Hörnig, 223-230. Neuwied/Darmstadt: Luchterhand.

Bourdieu, Pierre. 1983. Ökonomisches Kapital, kulturelles Kapital, soziales Kapital. In Soziale Ungleichheiten. Soziale Welt Sonderband 2, Hrsg. Reinhard Kreckel, 183-198. Göttingen: Schwartz.

Bourdieu, Pierre. 1987. Die feinen Unterschiede. Kritik der gesellschaftlichen Urteilskraft. Frankfurt a. M.: Suhrkamp.

Bourdieu, Pierre. 1992. Die verborgenen Mechanismen der Macht. Hamburg: VSA-Verlag.

Bourdieu, Pierre. 2013. Meditationen: Zur Kritik der scholastischen Vernunft. Frankfurt am Main: Suhrkamp Verlag.

Bourdieu, Pierre, und Jean-Claude Passeron. 1971. Die Illusion der Chancengleichheit. Untersuchungen zur Soziologie des Bildungswesens am Beispiel Frankreichs. Stuttgart: Klett.

Bourdieu, Pierre, und Jean-Claude Passeron. 1973. Grundlagen einer Theorie der symbolischen Gewalt. In Grundlagen einer Theorie der symbolischen Gewalt, Hrsg. Pierre Bourdieu, 7-87. Frankfurt am Main: Suhrkamp.

Bourdieu, Pierre, und Loic J. D. Wacquant. 1996. Reflexive Anthropogie. Frankfurt am Main: Suhrkamp.

Boyle, Jennifer D., und Elaine M. Boyle. 2013. Born just a few weeks early: Does it matter? Archives of Disease in Childhood Fetal and Neonatal Edition 98:F85-F88.

BQS (Institut für Qualität \& Patientensicherheit). 2012. BQS-Ergebnisse der Jahre 2001 bis 2008 -Archiv, online: http://www.bqs-institut.de/archiv.html (accessed: 13. 09. 2012).

Bradley, Robert H., und Robert F. Corwyn. 2002. Socioeconomic Status and Child Development. Annual Review of Psychology 53:371-399.

Brake, Anna, Helmut Bremer, und Andrea Lange-Vester. 2013. Empirisch Arbeiten mit Bourdieu. Theoretische und methodische Überlegungen, Konzeptionen und Erfahrungen. Weinheim: Beltz Juventa.

Bremer, Helmut. 2006. Die Transformation sozialer Selektivität. Soziale Milieus und Traditionslinien der Weiterbildungsteilnahme. In Soziale Milieus und der Wandel der Sozialstruktur. Die gesellschaftlichen Herausforderungen und die Strategien der sozialer Gruppen, Hrsg. Helmut Bremer und Andrea Lange-Vester, 186-211. Wiesbaden: VS Verlag für Sozialwissenschaften.

Brinbaum, Yaël, und Hector Cebolla Boado. 2007. The school careers of ethnic minority youth in France: Success or disillusion? Ethnicities 7:445-474.

Bründel, Heidrun, und Klaus Hurrelmann. 1996. Einführung in die Kindheitsforschung. Weinheim und Basel: Beltz Verlag.

Brüning, Andreas. 2005. Einfluss des Rauchens der Mütter in der Schwangerschaft auf die Gewichtsklassifikation der Neugeborenen und Risikostruktur der Raucherinnen unter Be- 
rücksichtigung ausgewählter biologischer und sozialer Merkmale. Dissertation, Universitätsmedizin Berlin.

Bühler, Christoph. 2007. Soziales Kapital und Fertilität. In Sozialkapital. Grundlagen und Anwendungen. Kölner Zeitschrift für Soziologie und Sozialpsychologie (Sonderheft 47), Hrsg. Axel Franzen und Markus Freitag, 397-419. Wiesbaden: VS Verlag für Sozialwissenschaften.

Bühling, Kai Joachim, und Wolfgang Friedmann. 2009. Intensivkurs Gynäkologie und Geburtshilfe. München: Urban \& Fischer.

Bundeszentrale für gesundheitliche Aufklärung (BZgA). 2003. Forschung und Praxis der Gesundheitsförderung, Bd. 20: Das Ernährungsverhalten Jugendlicher im Kontext ihrer Lebensstile. Eine empirische Studie. Köln: Bundeszentrale für gesundheitliche Aufklärung.

Bundeszentrale für gesundheitliche Aufklärung (BZgA). 2012. Ich bin dabei! - Vater werden. http://www.bzga.de/botmed_13510000.html. Zugegriffen: 27. Juli 2014.

Bundeszentrale für gesundheitliche Aufklärung (BZgA). 2014. Rundum - Schwangerschaft und Geburt. http://www.bzga.de/botmed_13500000.html. Zugegriffen: 27. Juli 2014.

Bung, Peter 2006. Schwangerschaft und Ernährung. In Die Geburtshilfe, Hrsg. Henning Schneider, Peter Husslein und Karl-Theo M. Schneider, 219-234. Heidelberg: Springer.

Cable, Noriko. 2015. Life Course Approach in Social Epidemiology: An Overview, Application and Future Implications. Journal of Epidemiology 24:347-352.

Calderon-Margalit, Ronit, Chunfang Qiu, Asher Ornoy, David S. Siscovick, und Michelle A. Williams. 2009. Risk of preterm delivery and other adverse perinatal outcomes in relation to maternal use of psychotropic medications during pregnancy. American Journal of $\mathrm{Ob}$ stetrics and Gynecology 201:e1-e8.

Cassel, John. 1976. The contribution of the social environment to host resistance. American Journal of Epidemiology 104:253-286.

Chen, Xinguang, Bonita Stanton, Jie Gong, Xiaoyi Fang, und Xiaoming Li. 2009. Personal Social Capital Scale: an instrument for health and behavioral research. Health Education Research 24:306-317.

Choté, Anoushka A., Gerit T. Koopmans, William K. Redekop, Christianne J. M. de Groot, Renske J. Hoefman, Vincent W. V. Jaddoe, Albert Hofman, Eric A.P. Steegers, Johan P. Mackenbach, Margo Trappenburg, und Marlen Foets. 2011. Explaining Ethnic Differences in Late Antenatal Care Entry by Predisposing - Enabling and Need Factors in the Netherlands: The Generation R Study. Maternal and Child Health Journal 15:689-699.

Chu, Shih-Ming, Ming-Horng Tsai, Fan-Ming Hwang, Jen-Fu Hsu, Hsuan Rong Huang, und Yu-Shu Huang. 2012. The relationship between attention deficit hyperactivity disorder and premature infants in Taiwanese: a case control study. BMC Psychiatry 12:85.

Cobb, Sidney. 1976. Social support as a moderator of life stress. Psychosomatic Medicine 38:300-314.

Coleman, James S. 1988. Social capital in the creation of human capital. American Journal of Sociology 94:94-120. 
Coleman, James S. 1990. Foundations of social theory. Cambridge, MA: Harvard University Press.

Coleman, James S. 1991. Grundlagen der Sozialtheorie. Band 1: Handlungen und Handlungssysteme. München: Oldenbourg Verlag.

Collatz, Jürgen, Hartmut Hecker, und Karl Oeter. 1983. Perinatalstudie Niedersachsen und Bremen. Soziale Lage, medizinische Versorgung, Schwangerschaftsverlauf und perinatale Mortalität. München: Urban und Schwarzenberg.

Collatz, Jürgen, Peter Malzahn, und Eberhard Schmidt. 1979. Erreichen die gesetzlichen Früherkennungsuntersuchungen für Säuglinge und Kleinkinder ihre Zielgruppen? Öffentliches Gesundheitswesen 41:173-190.

Corman, Hope, Theodore J. Joyce, und Michael Grossman. 1987. Birth Outcome Production Functions in the U.S. Journal of Human Resources 22:339-360.

Cramer, Colin, und Martin Harant. 2014. Inklusion - Interdisziplinäre Kritik und Perspektiven von Begriff und Gegenstand. Zeitschrift für Erziehungswissenschaft 17:639-659.

Currie, Janet, und Enrico Moretti. 2003. Mother's education and the intergenerational transmission of human capital: Evidence from college openings. The Quartely Journal of Economics 118:1495-1532.

Dahrendorf, Ralf. 1965. Bildung ist Bürgerrecht. Plädoyer für eine aktive Bildungspolitik. Hamburg: Cristian Wegner Verlag.

Dahrendorf, Ralf. 1979. Lebenschancen. Anläufe zur sozialen und politischen Theorie. Frankfurt am Main: Suhrkamp.

David, Matthias, Jürgen Pachaly, und Klaus Vetter. 2006. Perinatal outcome in Berlin (Germany) among immigrants from Turkey. Archives of Gynecology and Obstetrics 274:271278.

David, Matthias, und Jürgen Pachaly. 2010. Migrationshintergrund als geburtshilflicher Risikofaktor? Perinataldaten im Vergleich. In Kinder und Jugendliche mit Migrationshintergrund. Lebenswelten, Gesundheit und Krankheit, Hrsg. Theda Borde und Matthias David, 11-30. Frankfurt am Main: Mabuse-Verlag.

De Jong, Marjanneke, Marjolein Verhoeven, und Anneloes L. van Baar. 2012. School outcome, cognitive functioning, and behaviour problems in moderate and late preterm children and adults: A review. Seminars in fetal \& neonatal medicine 17:163-169.

Delvaux, Thérèse, Pierre Buekens, Isabelle Godin, und Michel Boutsen. 2001. Barriers to Prenatal Care in Europe. American Journal of Preventive Medicine 21:52-59.

Derose, Kathryn P., und Danielle M. Varda. 2009. Social Capital and Health Care Access: A systemativ Review. Medical Care Research and Review 66:272-306.

Dew, Paul C., V. James, Felix A. Okah, Jinwen Cai, und Gerald L. Hoff. 2007. The effect of health compromising behaviors on preterm births. Maternal and Child Health Journal 11:227-233. 
D'Hombres, Béatrice, Lorenzo Rocco, Marc Suhrcke, und Martin McKee. 2010. Does social capital determine health? Evidence from eight transition countries. Health economics 19:56-74.

Diefenbach, Heike. 2008. Bildungschancen und Bildungs(miss)erfolg von ausländischen Schülern oder Schülern aus Migrantenfamilien im System schulischer Bildung. In Bildung als Privileg, Hrsg. Rolf Becker und Wolfgang Lauterbach, 221-246.Wiesbaden: VS Verlag für Sozialwissenschaften.

Diefenbach, Heike. 2011. Der Bildungserfolg von Schülern mit Migrationshintergrund. In Lehrbuch der Bildungssoziologie, Hrsg. Rolf Becker, 49-473. Wiesbaden: VS Verlag für Sozialwissenschaften.

Diehl, Claudia, Christian Hunkler, und Cornelia Kristen. 2016. Ethnische Bildungsungleichheiten im Bildungsverlauf. Eine Einführung. In Ethnische Ungleichheiten im Bildungsverlauf. Mechanismen, Befunde, Debatten, Hrsg., Claudia Diehl, Christian Hunkler und Cornelia Kristen, 3-31. Wiesbaden: VS Verlag für Sozialwissenschaften.

Diekmann, Andreas. 2005. Empirische Sozialforschung. Grundlagen, Methoden, Anwendungen. Hamburg: rowohlts enzyklopädie.

Diekmann, Andreas. 2007. Dimensionen des Sozialkapitals. In Sozialkapital. Grundlagen und Anwendungen, Hrsg. Axel Franzen und Michael Freitag, Kölner Zeitschrift für Soziologie und Sozialpsychologie (Sonderheft 47), 47-65. Wiesbaden: VS Verlag für Sozialwissenschaften.

Diewald, Martin. 1991. Soziale Beziehungen: Verlust oder Liberalisierung? Soziale Unterstützung in informellen Netzwerken. Berlin: edition sigma.

Diewald, Martin. 2007. Arbeitsmarktungleichheiten und die Verfügbarkeit von Sozialkapital. Die Rolle von Gratifikationen und Belastungen. In Sozialkapital. Grundlagen und Anwendungen. Hrsg. Axel Franzen und Michael Freitag, Kölner Zeitschrift für Soziologie und Sozialpsychologie (Sonderheft 47), 183-210. Wiesbaden: VS Verlag für Sozialwissenschaften.

DiMaggio, Paul. 1982. Cultural capital and school success: The impact of status culture participation on the grades of U.S. high school students. American Sociological Review 47:189-201.

Dingeldey, Irene, und Ina Berninger. 2013. Familienlohn und Armutssicherung im Normalarbeitsverhältnis. Zur Einkommenssituation von Männern und Frauen in Ost- und Westdeutschland. Kölner Zeitschrift für Soziologie und Sozialpsychologie 65:669-696.

DiPrete, Thomas A., und Gregory M. Eirich. 2006. Cumulative Advantage as a Mechanism for Inequality: A Review of Theoretical and Empirical Developments. Annual Review of Sociology 32:271-297.

Ditton, Hartmut. 2008. Der Beitrag von Schule und Lehrern zur Reproduktion von Bildungsungleichheit. In Bildung als Privileg, Hrsg. Rolf Becker und Wolfgang Lauterbach, 247275. Wiesbaden: VS Verlag für Sozialwissenschaften. 
Ditton, Hartmut, Jan Krüsken, und Magdalena Schauenberg. 2005. Bildungsungleichheit der Beitrag von Familie und Schule. Zeitschrift für Erziehungswissenschaft 8:285-304.

Ditton, Hartmut, und Jan Krüsken. 2006. Der Übergang von der Grundschule in die Sekundarstufe I. Zeitschrift für Erziehungswissenschaft 9:348-372 .

Dollmann, Jörg. 2010. Türkischstämmige Kinder am ersten Bildungsübergang: Primäre und sekundäre Herkunftseffekte. Wiesbaden: VS Verlag für Sozialwissenschaften.

Doyle, Orla, Colm P. Harmon, James Heckman, und Richard E. Tremblay. 2009. Investing in Early Human Development: Timing and Economic Efficiency. Economics and Human Biology 7:1-6.

Dragano, Nico. 2007. Gesundheitliche Ungleichheit im Lebenslauf. Aus Politik und Zeitgeschichte (42): 18-25.

Dragano, Nico, und Siegrist Johannes. 2009. Die Lebenslaufperspektive gesundheitlicher Ungleichheit: Konzepte und Forschungsergebnisse. In Gesundheitliche Ungleichheit. Grundlagen, Probleme, Perspektiven, Hrsg. Matthias Richter und Klaus Hurrelmann, 181-194. Wiesbaden: VS Verlag für Sozialwissenschaften.

Drukker, Marjan, Stephen L. Buka, Charles Kaplan, Kwame McKenzie, und Jim Van Os. 2005. Social capital and young adolescents' perceived health in different sociocultural settings. Social Science \& Medicine 61:185-198.

Durkheim, Emile. 1970. Suicide: A Study in Sociology. Translated by John A. Spaulding and George Simpson. London: Routledge \& Kegan Paul.

Elder, Glen H. 1985. Perspectives on the Life Course. In Life Course Dynamics. Trajectories and Transitions 1968 - 1980, Hrsg. Glen H. Elder, 23-49. Ithaca: Cornell University Press.

Elder, Glen H. 1998. The Life Course and Human Development. In Theoretical Models of Human Development, Handbook of Child Psychology, Vol. 1, Hrsg. Richard M. Lerner, 939-991. New York: Wiley \& Sons.

Engle, William A., Kay M. Tomashek, und Carol Wallman. 2007. "Late-preterm" infants: a population at risk. Pediatrics 120:1390-401.

Erci, Behice. 2003. Barriers to utilization of prenatal care services in Turkey. Journal of Nursing Scholarship 35:269-273.

Ergöçmen, Banu. 1999. Maternal and child health. In Demographic and health survey Turkey 1998, Hrsg: Aykut Toros, 105-117. Ankara, Turkey: Ministry of Health/Mother Child Health and Family Planning.

Erhart, Michael, Nora Wille, und Ulrike Ravens-Sieberer. 2008. In die Wiege gelegt?: Gesundheit im Kindes- und Jugendalter als Beginn einer lebenslangen Problematik. In Health Inequalities, Hrsg. Ullrich Bauer, Uwe H. Bittlingmayer und Matthias Richter, 331-358. Wiesbaden: VS Verlag für Sozialwissenschaften.

Erikson, Robert, und John H. Goldthorpe. 1992. The Constant Flux. Oxford. 
Esser, Hartmut. 1999. Soziologie. Allgemeine Grundlagen. Frankfurt/New York: Campus Verlag.

Esser, Hartmut. 1999. Soziologie. Spezielle Grundlagen. Band 1: Situationslogik und Handeln. Frankfurt am Main: Campus Verlag.

Esser, Hartmut. 2000. Soziologie. Spezielle Grundlagen. Band 2: Die Konstruktion der Gesellschaft. Frankfurt/New York: Campus Verlag.

Esser, Hartmut. 2006. Migration, Sprache und Integration. Berlin: Wissenschaftszentrum Berlin für Sozialforschung (AKI-Forschungsbilanz 4).

Esser, Hartmut. 2008. Assimilation, ethnische Schichtung oder selektive Akkulturation. In Migration und Integration. Sonderheft 48 der Kölner Zeitschrift für Soziologie und Sozialpsychologie, Hrsg. Frank Kalter, 8-107. Wiesbaden: VS Verlag für Sozialwissenschaften.

Faltermaier, Toni. 1994. Gesundheitsbewusstsein und Gesundheitshandeln. Über den Umgang mit Gesundheit im Alltag. Weinheim: Beltz Verlag.

Faltermaier, Toni. 2009. Gesundheit: körperliche, psychische und soziale Dimensionen. In Handbuch der Gesundheitspsychologie und Medizinischen Psychologie, Hrsg. Jürgen Bengel und Matthias Jerusalem, 46-57. Göttingen: Hogrefe.

Faltermaier, Toni, Irene Kühnlein, und Martina Burda-Viering. 1998. Gesundheit im Alltag. Laienkompetenz in Gesundheitshandeln und Gesundheitsförderung. Weinheim: Juventa.

Fassaert, Thijs, Arlette E. Hesselink, und Arnoud P. Verhoeff. 2009. Acculturation and Use of Health Care Services by Turkish and Moroccan Migrants: A Cross-Sectional PopulationBased Study. BMC Public Health 9:332.

Feinstein, Jonathan. 1993. The relationship between socioeconomic status and health: A review of the literature. The Milbank Quarterly 71:279-322.

Flick, Uwe. 1998. Wann fühlen wir uns gesund? Subjektive Vorstellungen von Gesundheit und Krankheit. Weinheim: Juventa.

Fransen, Mirjam P., Marie-Louise Essink-Bot, Anke Oenema, Johan P. Mackenbach, Eric A.P. Steegers, und Hajo I.J. Wildschut. 2007. Ethnic differences in determinants of participation and nonparticipation in prenatal screening for Down syndrome: A theoretical framework, Prenatal Diagnosis 27:938-950.

Franzen, Axel, und Markus Freitag. 2007. Aktuelle Themen und Diskussionen der Sozialkapitalforschung. In Sozialkapital. Grundlagen und Anwendungen, Hrsg. Axel Franzen und Markus Freitag, 7-22. Wiesbaden: VS Verlag für Sozialwissenschaften.

Frick, Joachim R., und Janina Söhn. 2005. Das Sozio-Oekonomische Panel (SOEP) als Grundlage für Analysen zur Bildungslage von Personen mit Migrationshintergrund. In Migrationshintergrund von Kindern und Jugendlichen: Wege zur Weiterentwicklung der amtlichen Statistik. Band 14 der Reihe Bildungsreform des Bundesministeriums für Bildung und Forschung, Hrsg. Bundesministerium für Bildung und Forschung, 81-90. Bonn/Berlin. 
Friedrich, Engels. 1845. Die Lage der arbeitenden Klasse in England. Leipzig.

Fuchs, Marek, und Michaela Sixt. 2008. Die Bildungschancen von Aussiedlerkindern. SOEPpapers on Multidisciplinary Panel Data Research 105. Berlin: Deutsches Institut für Wirtschaftsforschung.

Fukuyama, Francis Y. 1997. Social capital, The Tanner lectures on human values. American Sociological Review 19:375-484.

Geißler, Rainer. 1994. Soziale Schichten und Lebenschancen in Deutschland. Stuttgart: Ferdinand Enke Verlag.

Georg, Werner. 2005. Die Reproduktion sozialer Ungleichheit im Lebenslauf. Zeitschrift für Soziologie der Erziehung und Sozialisation 25:178-197.

Gerhards, Jürgen, Jörg Rössel, Claudia Beckert-Zieglschmid, und Janet Bennat. 2004. Geschlecht, Lebensstile und Ernährung. In Soziale Benachteiligung und Geschlecht bei Kindern und Jugendlichen, Hrsg. Monika Jungbauer-Gans und Peter Kriwy, 151-176. Wiesbaden: VS Verlag für Sozialwissenschaften.

Gerlinger, Thomas. 2006. Historische Entwicklung und theoretische Perspektiven der Gesundheitssoziologie. In Soziologie der Gesundheit. Sonderband 46 der Kölner Zeitschrift für Soziologie und Sozialpsychologie, Hrsg. Claus Wendt und Christof Wolf, 34-56. Wiesbaden: VS Verlag für Sozialwissenschaften.

Girard, Alain, und Henri Bastide. 1963. La stratification sociale et al démocratisation de l'enseignement, Population 18:435-72.

Gisselmann, Marit Dahlen, und Orjan Hemström. 2008. The contribution of maternal working conditions to socio-economic inequalities in birth outcome. Social Science \& Medicine 66:1297-1309.

Glaesmer, Heide, Ulla Wittig, Elmar Braehler, Alexandra Martin, Ricarda Mewes, und Winfried Rief. 2011. Health care utilization among first and second generation immigrants and native-born Germans: a population-based study in Germany. International Journal Public Health 56:541-548.

Goedhart, Geertje, Manon van Eijsdena, Marcel F. van der Wal, und Gouke J. Bonsel. 2008. Ethnic differences in term birthweight: the role of constitutional and environmental factors. Paediatric and Perinatal Epidemiology 22:360-368.

Goldberg, Susan, und Barbara DiVitto. 2002. Parenting Children Born Preterm. In: Handbook of Parenting. Vol. 1: Children and Parenting. Second Edition, Hrsg. Marc H. Bornstein, 329-354. Mahwah, NJ, US: Lawrence Erlbaum Associates Publishers.

Gomolla, Mechtild, und Frank-Olaf Radtke. 2002. Institutionelle Diskriminierung. Die Herstellung ethnischer Differenz in der Schule. Opladen: Leske + Budrich.

Gresch, Cornelia, und Michael Becker. 2010. Sozial und leistungsbedingte Disparitäten im Übergangsverhalten bei türkischstämmigen Kindern und Kindern aus (Spät-)Aussiedlerfamilien. In Der Übergang von der Grundschule in die weiterführende Schule - Leistungsgerechtigkeit und regionale, soziale und ethnisch-kulturelle Disparitä- 
ten: Zusammenfassung der zentralen Befunde, Hrsg. Jürgen Baumert, Kai Maaz, Cornelia Gresch und Nele McElvany, 181-200. Bonn, Berlin: BMBF.

Günter, Hans-Heinrich, Alexander Scharf, Peter Hillemanns, Paul Wenzlaff, und Holger Maul. 2007. Schwangerschaften ohne Vorsorgeuntersuchung - welche Frauen sind potenziell betroffen, welche Risiken ergeben sich für das Kind? Zeitschrift für Geburtshilfe und Neonatalogie 211:27-32.

Haisken-DeNew, John P., und Joachim R. Frick. 2005. DTC - Desktop Companion to the German Socio-Economic Panel (SOEP). Berlin.

Harris, Malinda N., Robert G. Voigt, William J. Barbaresi, Gretchen Voge, Jill M. Killian, Amy L. Weaver, Christopher E. Colby, William A. Carey, und Slavica K. Katusic. 2013. ADHD and Learning Disabilities in Former Late Preterm Infants: A Population-Based Birth Cohort. Pediatrics 132: e630-e636.

Harrison, Patricia A., und Abbey C. Sidebottom. 2009. Alcohol and drug use before and during pregnancy: An examination of use patterns and predictors of cessation. Maternal and Child Health Journal 13:386-394.

Hart, Betty, und Todd R. Risley. 2003. The early catastrophe: The 30 million word gap by age 3. American Educator 27:4-9.

Hartung, Susanne, Sabine Kluwe, Diana Sahrai, und Klaus Hurrelmann. 2010. Elternbildung und Elternpartizipation in Settings. Eine programmspezifische und vergleichende Analyse von Interventionsprogrammen in Kita, Schule und Kommunen. Abschlussbericht, Bielefelder Evaluation von Elternedukationsprogrammen (BEEP). Bielefeld, Hannover.

Haug, Sonja. 1997. Soziales Kapital. Ein kritischer Überblick über den aktuellen Forschungsstand. Arbeitsbericht Nr. 15. Mannheim: Mannheimer Zentrum für Europäische Sozialforschung (MZES).

Haug, Sonja. 2007. Soziales Kapital als Ressource im Kontext von Migranten und Integration. In Soziale Netzwerke und Soziale Ungleichheit. Zur Rolle von Sozialkapital in modernen Gesellschaften, Hrsg. J. Lüdicke und Martin Diewald, 85-111. Wiesbaden: VS Verlag für Sozialwissenschaften.

Häußermann, Harmut, und Walter Siebel. 1996. Soziologie des Wohnens. Eine Einführung in Wandel und Ausdifferenzierung des Wohnens. Weinheim, München: Juventa.

Hawe, Penelope, und Alan Shiell. 2000. Social capital and health promotion: a review. Social Science \& Medicine 51:871-885.

Heaney, Catherine A., und Barbara A. Israel. 2008. Social networks and social support. In Health behavior and health education. Theory, research and practice, Hrsg. Karen Glanz, Barbara K. Rimer und Kasisomayajula Viswanath, 189-210. San Francisco: Jossey-Bass.

Heath, Antony, und Yaël Brinbaum. 2007. Explaining ethnic inequalities in educational attainment. Ethnicities 7:291-305.

Helfferich, Cornelia, Anneliese Hendel-Kramer, und Heike Kindworth. 2003. Gesundheit alleinerziehender Mütter und Väter. Gesundheitsberichterstattung des Bundes 14:3-27. 
Henkel, Dieter, Uwe Zemlin, und Peer Dornbusch. 2003. Sozialschicht und Konsum von Alkohol und Tabak im Bundesgesundheitssurvey 1998. SUCHT - Zeitschrift für Wissenschaft und Praxis 49:306-311.

Henz, Ursula, und Ineke Maas. 1995. Chancengleichheit durch die Bildungsexpansion. Kölner Zeitschrift für Soziologie und Sozialpsychologie 47:605-633.

Hillmert, Steffen. 2008. Soziale Ungleichheit im Bildungsverlauf: Zum Verhältnis von Bildungsinstitutionen und Entscheidungen. In Bildung als Privileg, Hrsg. Rolf Becker und Wolfgang Lauterbach, 75-102.Wiesbaden: VS Verlag für Sozialwissenschaften.

Hölling, Heike, Panagiotis Kamtsiuris, Michael Lange, Wulf Thierfelder, Michael Thamm, und Robert Schlack. 2007. Der Kinder- und Jugendgesundheitssurvey (KiGGS): Studienmanagement und Durchführung der Feldarbeit. Bundesgesundheitsblatt - Gesundheitsforschung - Gesundheitsschutz 50:557-566.

Honein, Margaret A., Russell S. Kirby, Robert E. Meyer, Jian Xing, Nyasha I. Skerrette, Nataliya Yuskiv, Lisa Marengo, Joann R. Petrini, Michael J. Davidoff, Cara T. Mai, Charlotte M. Druschel, Samara Viner-Brown, und Lowell E. Sever. 2009. The association between major birth defects and preterm birth. Maternal and Child Health Journal 13:164175.

House, James S., Debra Umberson, und Karl R. Landis. 1988. Structures and processes of social support. Annual Review of Sociology 14:293-318.

Hradil, Stefan. 2006. Was prägt das Krankheitsrisiko: Schicht, Lage, Lebensstil? In Gesundheitliche Ungleichheit, Hrsg. Matthias Richter und Klaus Hurrelmann, 33-52. Wiesbaden: VS Verlag für Sozialwissenschaften.

Hsin, Amy. 2012. Is Biology Destiny? Birth Weight and Differential Parental Treatment. Demography 49:1385-1405.

Huch, Renate. 2006. Beratungsgrundlagen zur Lebensführung in der Schwangerschaft. In Die Geburtshilfe, Hrsg. Henning Schneider, Peter Husslein und Karl-Theo M. Schneider, 199-218. Heidelberg: Springer.

Hurrelmann, Klaus. 1988. Sozialisation und Gesundheit. Weinheim und München: Juventa Verlag.

Hurrelmann, Klaus. 2006. Gesundheitssoziologie. Eine Einführung in sozialwissenschaftliche Theorien von Krankheitsprävention und Gesundheitsförderung. Weinheim und München: Juventa.

Hurrelmann, Klaus. 2008. Eltern sind Laienerzieher. Frankfurter Rundschau vom 14.02.2008, Nr. 38, 64. Jahrgang.

Huschek, Doreen, Helga A. G. de Valk, und Aart C. Liefbroer. 2012. Partner Choice Patterns Among the Descendants of Turkish Immigrants in Europe. European Journal of Population 28:241-268.

Hustinx, Paul Willem J. 2002. School Careers of Pupils of Ethnic Minority Background after the Transition to Secondary Education: Is the Ethnic Factor Always Negative? Educational Research \& Evaluation 8:169-195. 
Hyman, Herbert H. 1953. The Values Systems of Different Classes: A Social Psychological Contribution to the Analysis of Stratification, In Class, Status and Power, Hrsg. Reinhard Bendix und Martin S. Lipset, 426-442. New York: Free Press.

Institut für Qualität \& Patientensicherheit (BQS). 2012. BQS-Ergebnisse der Jahre 2001 bis 2008. Archiv. http://www.bqs-institut.de/archiv.html. Zugegriffen: 13. Sept. 2012.

Jackson, Michelle. 2013. Determined to succeed? Performance versus choice in educational attainment. Stanford: Stanford University Press.

Jäkel, Julia, Nicole Baumann, und Dieter Wolke. 2013. Effects of Gestational Age at Birth on Cognitive Performance: A Function of Cognitive Workload Demands. PLoS ONE 8:e65219.

Jäkel, Julia, Dieter Wolke, und Birgit Leyendecker. 2012. Resilienz im Vorschulalter: Wie stark kann die familiäre Leseumwelt biologische und soziokulturelle Entwicklungsrisiken kompensieren? Zeitschrift für Familienforschung 24:148-159.

Janevic, Teresa, David A. Savitz, und Mary Janevic. 2011. Maternal education and adverse birth outcomes among immigrant women to the United States from Eastern Europe: A test of the healthy migrant hypothesis. Social Science \& Medicine 73:429-435.

Johnson, Samantha, Camilla Gilmore, Ian Gallimore, Julia Jaekel, und Dieter Wolke. 2015. Education Professionals' Knowledge of Preterm Birth. Developmental Medicine \& Child Neurology 57:571-577.

Jonsson, Jan O., und Frida Rudolphi. 2010. Weak Performance - Strong Determination: School Achievement and Educational Choice among Children of Immigrants in Sweden. European Sociological Review 27:1-22.

Jungbauer-Gans, Monika. 2002. Ungleichheit, soziale Beziehungen und Gesundheit. Wiesbaden: Westdeutscher Verlag.

Jungbauer-Gans, Monika. 2006. Soziale und kulturelle Einflüsse auf Krankheit und Gesundheit. In Soziologie der Gesundheit. Sonderband 46 der Kölner Zeitschrift für Soziologie und Sozialpsychologie, Hrsg. Claus Wendt und Christof Wolf, 86-108. Wiesbaden: VS Verlag für Sozialwissenschaften.

Jungbauer-Gans, Monika, und Peter Kriwy. 2004. Ungleichheit und Gesundheit von Kindern und Jugendlichen. In Soziale Benachteiligung und Gesundheit bei Kindern und Jugendlichen, Hrsg. Monika Jungbauer-Gans und Peter Kriwy, 9-24. Wiesbaden: VS Verlag für Sozialwissenschaften.

Kahl, Heidrun. 2010. Kindergesundheit - ein Ost-West-Vergleich und der Trend der letzten 20 Jahre. In Freidenker 69:28-35.

Kalies, Helen, und Rüdiger von Kries. 2002. Gesundheit im Kindesalter. Kurzbericht über die Ergebnisse der Schuleingangsuntersuchungen 1998/99-2000/01 in Bayern. München: Bayerisches Staatsministerium für Gesundheit Ernährung und Verbraucherschutz.

Kalter, Frank. 2008. Stand, Herausforderungen und Perspektiven der empirischen Migrationsforschung. In Migration und Integration. Sonderheft 48 der Kölner Zeitschrift für Sozio- 
logie und Sozialpsychologie, Hrsg. Frank Kalter, 12-36. Wiesbaden: VS Verlag für Sozialwissenschaften.

Kamtsiuris, Panagiotis, Eckardt Bergmann, Petra Rattay, und Martin Schlaud. 2007a. Inanspruchnahme medizinischer Leistungen. Ergebnisse des Kinder- und Jugendgesundheitssurveys (KiGGS). In Bundesgesundheitsblatt - Gesundheitsforschung - Gesundheitsschutz 50:836-850.

Kamtsiuris, Panagiotis, Michael Lange, und Angelika Schaffrath Rosario. 2007b. Der Kinderund Jugendgesundheitssurvey (KiGGS): Stichprobendesign, Response und NonresponseAnalyse. Bundesgesundheitsblatt - Gesundheitsforschung - Gesundheitsschutz 50:547556.

Kao, Grace, und Marta Tienda. 1998. Educational aspirations of minority youth. American Journal of Education 106: 349-384.

Kawachi, Ichiro, Bruce P. Kennedy, und Roberta Glass. 1999. Social capital and self-rated: a contextual analysis. American Journal of Public Health 89:1187-1183.

Kemptner, Daniel, und Jan Marcus. 2012. Spillover effects of maternal education on child's health and health behavior. Review of Economics of the Household 11:29-52.

Khashan, Ali S., Philip N. Baker, und Louise C. Kenny. 2010. Preterm birth and reduced birthweight in first and second teenage pregnancies: A register-based cohort study. $B M C$ Pregnancy and Childbirth 10:1-36.

Kirschner, Wolf, Horst Halle, und Marc-André Pogonke. 2009. Kosten der Früh- und Nichtfrühgeburten und die Effektivität und Effizienz von Präventionsprogrammen am Beispiel von BabyCare. Eine Schätzung auf der Grundlage der DRG unter Berücksichtigung der Primäraufnahmen in der Neonatologie. Prävention und Gesundheitsförderung 4:41-50.

Kitze, Katharina, und Bettina Kuske. 2008. Schwangerschaft und Geburt. In Medizinische Psychologie und Medizinische Soziologie von A bis Z, Hrsg. Hendrik Berth, Friedrich Balck, und Elmar Bähler, 417-420. Göttingen: Hogrefe.

Klauer, Thomas. 2009. Soziale Unterstützung. In Handbuch der Gesundheitspsychologie und Medizinischen Psychologie, Hrsg. Jürgen Bengel und Matthias Jerusalem, 80-85. Göttingen: Hogrefe.

Klein, Thomas, Sven Schneider, und Hannelore Löwel. 2001. Bildung und Mortalität. Die Bedeutung gesundheitsrelevanter Aspekte des Lebensstils. Zeitschrift für Soziologie 30:384-400.

Klocke, Andreas. 2004. Soziales Kapital als Ressource für Gesundheit im Jugendalter. In Soziale Benachteiligung und Gesundheit bei Kindern und Jugendlichen, Hrsg. Monika Jungbauer-Gans und Peter Kriwy, 85-96. Wiesbaden: VS Verlag für Sozialwissenschaften.

Klocke, Andreas, und Ulrich Becker. 2003. Die Lebenswelt Familie und ihre Auswirkungen auf die Gesundheit von Jugendlichen. In Jugendgesundheitssurvey - Internationale Vergleichsstudie im Auftrag der Weltgesundheitsorganisation WHO, Hrsg. Klaus Hurrel- 
mann, Andreas Klocke und Wolfgang Melzer,183-242.Weinheim und München: Juventa Verlag.

Klocke, Andreas, und Klaus Hurrelmann. 1995. Armut und Gesundheit. Inwieweit sind Kinder und Jugendliche betroffen? Zeitschrift für Gesundheitswissenschaften 2:138-151.

Kohler, Ulrich, Kristian Berndt, und Anders Holm. 2011. Comparing coefficients of nested nonlinear probability models. The Stata Journal 11:420-438.

Kolleck, Bernd, Johannes Korporal, und Angela Zink. 1979. Totgeburtlichkeit und Säuglingssterblichkeit ausländischer Kinder in West-Berlin. Gynäkologe 12:181-190.

Koller, Daniela, Nicholas Lack, und Andreas Mielck. 2008. Soziale Unterschiede bei der Inanspruchnahme der Schwangerschaftsvorsorgeuntersuchungen, beim Rauchen der Mutter während der Schwangerschaft und beim Geburtsgewicht des Neugeborenen. Empirische Analyse auf Basis der Bayerischen Perinatal-Studie. Das Gesundheitswesen 71:10-18.

Koob, Dirk. 2007. Sozialkapital zur Sprache gebracht. Eine bedeutungstheoretische Perspektive auf ein sozialwissenschaftliches Begriffs- und Theorieproblem. Göttingen: Universitätsverlag.

Kratzmann, Jens, und Thorsten Schneider. 2009. Soziale Ungleichheiten beim Schulstart. Empirische Untersuchungen zur Bedeutung der sozialen Herkunft und des Kindergartenbesuchs auf den Zeitpunkt der Einschulung. Kölner Zeitschrift für Soziologie und Sozialpsychologie 61:211-234.

Kreckel, Reinhard. 1992. Politische Soziologie der sozialen Ungleichheit. Frankfurt am Main/New York: Campus.

Krenz, Astrid. 2008. Theorie und Empirie über den Wirkungszusammenhang zwischen sozialer Herkunft, kulturellem und sozialem Kapital, Bildung und Einkommen in der Bundesrepublik Deutschland. SOEPpapers Nr. 128. Berlin.

Kristen, Cornelia. 1999. Bildungsentscheidungen und Bildungsungleichheit. Ein Überblick über den Forschungsstand. Mannheim: Mannheimer Zentrum für Europäische Sozialforschung (Arbeitspapiere 5).

Kristen, Cornelia. 2002. Hauptschule, Realschule oder Gymnasium? Kölner Zeitschrift für Soziologie und Sozialpsychologie 54:534-552.

Kristen, Cornelia. 2008. Schulische Leistungen von Kindern aus türkischen Familien am Ende der Grundschulzeit: Befunde aus der IGLU-Studie, Kölner Zeitschrift für Soziologie und Sozialpsychologie, Sonderheft 48:230-251.

Kristen, Cornelia, und Jörg Dollmann. 2009. Sekundäre Effekte der ethnischen Herkunft? Kinder aus türkischen Familien am ersten Bildungsübergang. Zeitschrift für Erziehungswissenschaft 12:205-229.

Kristen, Cornelia, und Jörg Dollmann. 2010. Sekundäre Effekte der ethnischen Herkunft. Kinder aus türkischen Familien am ersten Bildungsübergang. In Vom Kindergarten bis zur Hochschule. Die Generierung von ethnischen und sozialen Disparitäten in der Bildungsbiographie, Hrsg. Birgit Becker, 117-144.Wiesbaden: VS Verlag für Sozialwissenschaften. 
Kristen, Cornelia, Aileen Edele, Frank Kalter, Irena Kogan, Benjamin Schulz, Petra Stanat, und Gisela Will. 2011. The education of migrants and their children across the life course. Zeitschrift für Erziehungswissenschaft 14:121-137.

Kristen, Cornelia, und Nadja Granato. 2007. The educational attainment of the second generation in Germany. Social origins and ethnic inequality. Ethnicities 7:343-366.

Kroll, Lars Eric, und Thomas Lampert. 2007. Sozialkapital und Gesundheit in Deutschland. Das Gesundheitswesen 67:120-127.

Krueger, Paul M., und Theresa O. Scholl. 2001. Adequacy of prenatal care and pregnancy outcome. The Journal of the American Osteopathic Association 100:485-492.

Krüger-Hemmer, Christiane. 2016. Bildung. In Datenreport 2016. Ein Sozialbericht fur die Bundesrepublik Deutschland, Hrsg. Statistisches Bundesamt/Wissenschaftszentrum Berlin für Sozialforschung, 79-101. Bonn: Bundeszentrale für politische Bildung.

Kühnel, Steffen-Matthias, und Dagmar Krebs. 2014. Statistik für die Sozialwissenschaften. Grundlagen, Methoden, Anwendungen. Reinbek bei Hamburg: Rowohlt Taschenbuch Verlag.

Kuhrs, Ema, Volker Winkler, und Heiko Becher. 2012. Risk factors for cardiovascular and cerebrovascular diseases among ethnic Germans from the former Soviet Union: results of a nested case-control study. BMC Public Health 12:1-9.

Kuper, Hannah, Michael Marmot, und Harry Hemingway. 2002. Systematic review of prospective cohort studies of psychosocial factors in the etiology and prognosis of coronary heart disease. Seminars in Vascular Medicine 23:267-314.

Kurth, Bärbel-Maria. 2007. Der Kinder- und Jugendgesundheitssurvey (KiGGS): Ein Überblick über Planung, Durchführung und Ergebnisse unter Berücksichtigung von Aspekten eines Qualitätsmanagements. Bundesgesundheitsblatt - Gesundheitsforschung - Gesundheitsschutz 50:533-546.

Kurz, Karin, und Sten Becker. 2017. Health risk ,single-mother family‘? Towards an explanation of their low participation rate in Germany'a early health screening programme for children. In Parents in the Spotlight. Parenting Practices and Support from a Comparative Perspective (Journal of Family Research, Special Issue 11), Hrsg. Tanja Betz, Michael-Sebastion Honig und Ilona Ostner, 171-194. Opladen: Barbara Budrich Verlag.

Kurz, Karin, und Wiebke Paulus. 2008. Übergänge im Grundschulalter: Die Formation elterlicher Bildungsaspirationen. In Die Natur der Gesellschaft: Verhandlungen des 33. Kongresses der Deutschen Gesellschaft für Soziologie in Kassel 2006, Hrsg. Karl-Siegbert Rehberg, 5489-5503. Frankfurt: Campus Verlag.

Kynø, Nina M., Ingrid Helen Ravn, Rolf Lindemann, Nina Aarhus Smeby, Anne Mari Torgersen, und Tonje Gundersen. 2013. Parents of preterm-born children; sources of stress and worry and experiences with an early intervention programme - a qualitative study. BMC Nursing 12:1-12.

Lamb, Michael E. 1975. Fathers: Forgotten contributors to child development. Human Development 18: 245-266. 
Lampert, Thomas, Christine Hagen, und Boris Heizmann. 2010. Gesundheitliche Ungleichheit bei Kindern und Jugendlichen in Deutschland. Beiträge zur Gesundheitsberichtserstattung des Bundes. Berlin: Robert Koch-Institut.

Lampert, Thomas, und Lars Eric Kroll. 2005. Einfluss der Einkommensposition auf die Gesundheit und Lebenserwartung. DIW Discussion Papers Nr. 527. Berlin.

Lampert, Thomas, und Lars E. Kroll. 2006. Messung des sozioökonomischen Status in sozialepidemiologischen Studien. In Gesundheitliche Ungleichheit. Grundlagen, Probleme, Perspektiven, Hrsg. Matthias Richter und Klaus Hurrelmann, 297-319. Wiesbaden: VS Verlag für Sozialwissenschaften.

Lampert Thomas, und Sabine M. List. 2010. Gesundheitsrisiko Passivrauchen (Gesundheitsberichterstattung des Bundes Kompakt 3). Berlin: Robert Koch Institut.

Lampert, Thomas, und Matthias Richter. 2006. Gesundheitliche Ungleichheit bei Kindern und Jugendlichen. In Gesundheitliche Ungleichheit. Grundlagen, Probleme, Perspektiven, Hrsg. Matthias Richter und Klaus Hurrelmann, 209-230. Wiesbaden: VS Verlag für Sozialwissenschaften.

Lampert, Thomas, Anke-Christine Saß, Michael Häfelinger, und Thomas Ziese. 2005. Armut, soziale Ungleichheit und Gesundheit, Beiträge zur Gesundheitsberichtserstattung des Bundes (Expertise des Robert Koch Instituts zum 2. Armuts- und Reichtumsbericht der Bundesregierung).

Lampert, Thomas, und Thomas Ziese. 2005. Armut, soziale Ungleichheit und Gesundheit. Expertise des Robert Koch-Instituts zum 2. Armuts- und Reichtumsbericht der Bundesregierung. Berlin: Robert Koch Institut.

Lanfranchi, Andrea, und Andrea Burgener Woeffray. 2013. Familien in Risikosituationen durch frühkindliche Bildung erreichen. In Handbuch Frühkindliche Bildungsforschung, Hrsg. Margrit Stamm und Doris Edelmann, 603-616. Wiesbaden: VS Verlag für Sozialwissenschaften.

Lange, Michael, Panagiotis Kamtsiuris, Cornelia Lange, Angelika Schaffrath Rosario, Heribert Stolzenberg, und Thomas Lampert. 2007. Messung soziodemographischer Merkmale im Kinder- und Jugendgesundheitssurvey (KiGGS) und ihre Bedeutung am Beispiel der Einschätzung des allgemeinen Gesundheitszustands. Bundesgesundheitsblatt - Gesundheitsforschung - Gesundheitsschutz 50:578-589.

Langness, Andrea. 2007. Prävention bei sozial benachteiligten Kindern. Eine Studie zur Inanspruchnahme von Früherkennungsuntersuchungen. Zugl.: Bielefeld, Univ., Diss. 1. Aufl. Bern: Huber (Studien zur Gesundheits- und Pflegewissenschaft).

Larroque, Beatrice, Pierre-Yves Ancel, Laetitia Marchand-Martin, Gilles Cambonie, Jeanne Fresson, Véronique Pierrat, Jean-Christophe Rozé, Loic Marpeau, Gerard Thiriez, Corinne Alberge, Gérard Bréart, Monique Kaminski, und Stéphane Marret. 2011. Special Care and School Difficulties in 8-Year-Old Very Preterm Children: The Epipage Cohort Study. PLoS ONE 6: e21361. 
Lengerer, Andrea. 2012. Die soziale Selektivität des partnerschaftlichen Zusammenlebens im Wandel. Eine kohortenbezogene Analyse kumulierter Mikrozensen. Kölner Zeitschrift für Soziologie und Sozialpsychologie 64:247-275.

Leyendecker, Birgit, und Axel Schölmerich. 2005. Familie und kindliche Entwicklung im Vorschulalter: Der Einfluss von Kultur und sozioökonomischen Faktoren. In Familie, Akkulturation und Erziehung. Migration zwischen Eigen- und Fremdkultur, Hrsg. Urs Fuhrer und Haci-Halil Uslucan, 17-39. Stuttgart: Verlag W. Kohlhammer.

Lin, Nan, Walter M. Ensel, Ronald S. Simeone, und Wen Kuo. 1979. Social Support, Stressful Life Events, and Illness: A Model and an Empirical Test. Journal of Health and Social Behaviour 20:108-119.

Lindenberg, Siegwart. 2001. Social rationality versus rational egoism. In Handbook of sociological theory, Hrsg. Jonathan H. Turner, 635-668. New York/Boston/Dordrecht/ London/Moscow: Kluwer Academic/Plenum.

Lindstrom, Martin. 2005. Social capital, the miniaturization of community and high alcohol consumption: a population-based study. Alcohol and Alcoholism 40:556-562.

Lohmann, Henning, Christa K. Spieß, Olaf Groh-Samberg, und Jürgen Schupp. 2009. Analysepotenziale des Sozio-oekonomischen Panels (SOEP) für die empirische Bildungsforschung. Zeitschrift für Erziehungswissenschaft 12:252-280.

Lüdicke, Jörg, und Martin Diewald. 2007. Soziale Netzwerke und Soziale Ungleichheit. Zur Rolle von Sozialkapital in modernen Gesellschaften. Wiesbaden: VS Verlag für Sozialwissenschaften.

Lüdtke, Oliver, Alexander Robitzsch, Ulrich Trautwein, und Olaf Köller. 2007. Umgang mit fehlenden Werten in der psychologischen Forschung. Probleme und Lösungen. Psychologische Rundschau 58:103-117.

Lumley, Judith. 1993. The epidemiology of preterm birth. Baillibre's Clinical Obstetrics and Gynaecology 7:477-498.

Lundborg, Petter. 2005. Social capital and substance use among Swedish adolescents -- an explorative study. Social Science \& Medicine 61:1151-1158.

Luo, Zhong Cheng, Russel Wilkins, und Michael S. Kramer. 2004. Disparities in pregnancy outcomes according to marital and cohabitation status. Obstetrics \& Gynecology. 103:1300-1307.

Lynch, Jamie L. 2011. Infant Health, Race/ Ethnicity, and Early Educational Outcomes Using the ECLS-B. Sociological Inquiry 81:499-526.

MacDorman, Marian F. 2011. Race and Ethnic Disparities in Fetal Mortality, Preterm Birth, and Infant Mortality in the United States: an Overview. Seminars in Perinatology 35:200-208.

Martius, Joachim A., Thomas Steck, Martin K. Oehler, und Karl-H. Wulf. 1998. Risk factors associated with preterm $(<37+0$ weeks $)$ and early preterm birth $(<32+0$ weeks $)$ : Univariate and multivariate analysis of 106345 singleton births from the 1994 statewide per- 
inatal survey of Bavaria. European Journal of Obstetrics \& Gynecology and Reproductive Biology 80:183-189.

Mayer, Karl Ulrich. 1987. Lebenslaufforschung. In Methoden der Biographie- und Lebenslaufforschung, Hrsg. Wolfgang Voges, 51-73. Opladen: Westdeutscher Verlag.

Mayer, Karl Ulrich. 1990. Lebensverläufe und sozialer Wandel. Anmerkungen zu einem Forschungsprogramm. In: Lebensverläufe und sozialer Wandel, Hrsg. Karl Ulrich Mayer, 7 21. Opladen: Westdeutscher Verlag.

Mersmann, Heiner. 1998. Gesundheit von Schulanfängern. Die Auswirkungen sozialer Benachteiligungen. In Forschung und Praxis der Gesundheitsförderung. Band 3 Gesundheit von Kindern, Epidemiologische Grundlagen, Expertentagung, Hrsg. Bundeszentrale für gesundheitliche Aufklärung, 60-78. Köln.

Meulemann, Heiner. 1990. Schullaufbahnen, Ausbildungskarrieren und die Folgen im Lebensverlauf. In Lebensverläufe und sozialer Wandel, Hrsg. Karl Ulrich Mayer, 89-117. Opladen: Westdeutscher Verlag.

Meyer-Nürnberger, Monika. 2002. Gesundheit von Kindern. Eine Sichtung regionaler und lokaler Gesundheitsberichterstattung. Bundesgesundheitsblatt - Gesundheitsforschung Gesundheitsschutz 45:859-865.

Mielck, Andreas. 2005. Soziale Ungleichheit und Gesundheit. Einführung in die aktuelle Diskussion. Bern: Hans Huber.

Mielck, Andreas, und Kim Bloomfield. 2001.Verringerung der Einkommensungleichheit und Verstärkung des sozialen Kapitals: Neue Aufgaben der sozial-epidemiologischen Forschung. Gesundheitswesen 63:18-23.

Milewski, Nadja, und Frederik Peters. 2014. Too low or too high? On birthweight differentials of immigrants in Germany. Comparative Population Studies 39:3-22.

Ministerium für Justiz, Gesundheit und Soziales. 2005. Bericht zur Gesundheit und gesundheitlichen Versorgung von Einschulkindern im Saarland. Saarbrücken.

Mirowsky, John, und Catherine E. Ross. 2003. Education, social status, and health. New York: Aldine De Gruyter.

Mirowsky, John, und Catherine E. Ross. 2007. Life course trajectories of perceived control and their relationship to education. American Journal of Sociology 112:1339-1382.

Misra, Dawn P., Cleopatra Caldwell, Alford A. Young Jr., und Sara Abelson. 2010. Do fathers matter? Paternal contributions to birth outcomes and racial disparities. American Journal of Obstetrics \& Gynecology 202:99-100.

Mohensi, Mohabbat, und Martin Lindstrom. 2007. Social capital, trust in the health-care system and self-rated health: the role of access to health care in a population-based study. Social Science \& Medicine 64:1373-1383.

Mood, Carina. 2010. Logistic regression: Why we cannot do what we think we can do, and what we can do about it. European Sociological Review 26:67-82. 
Moore, Keith L., und T. Vidhya N. Persaud. 2007. Embryologie. Entwicklungsstadien Frühentwicklung - Organogenese - Klinik. München: Urban \& Fischer.

Motakef, Mona. 2006. Das Menschenrecht auf Bildung und der Schutz vor Diskriminierung. Exklusionsrisiken und Inklusionschancen. Berlin: Deutsches Institut für Menschenrechte.

Mozurkewich, Ellen L., Barbara Luke, Michael Avni, und Frederic M. Wolf. 2000. Working conditions and adverse pregnancy outcome: a meta-analysis. Obstetrics and Gynecology 95:623-635.

Mueller, Ulrich, und Monika Heinzel-Gutenbrunner. 2001. Krankheiten und Beschwerden (subjektive Gesundheit) unter Bewertung der eigenen Gesundheit. BIB Bundesinstitut für Bevölkerungsforschung beim Statistischen Bundesamt Heft 102c.

Müller, Hans-Peter. 2014. Pierre Bourdieu - Eine systematische Einführung. Berlin: Suhrkamp Verlag.

Müller, Walter. 1975. Familie, Schule, Beruf. Analysen zur sozialen Mobilität und Statuszuweisung in der Bundesrepublik, Studien zur Sozialwissenschaft 25, Opladen: Westdeutscher Verlag.

Müller, Andrea G., und Petra Stanat. 2006. Schulischer Erfolg von Schülerinnen und Schülern mit Migrationshintergrund: Analysen zur Situation von Jugendlichen aus der ehemaligen Sowjetunion und der Türkei. In Herkunftsbedingte Disparitäten im Bildungswesen: Differenzielle Bildungsprozesse und Probleme der Verteilungsgerechtigkeit, Hrsg. Jürgen Baumert, Petra Stanat und Rainer Watermann, 221-256. Wiesbaden: VS Verlag für Sozialwissenschaften.

Müller-Rieckmann, Edith. 2006. Das frühgeborene Kind in seiner Entwicklung. Eine Elternberatung. München: Ernst Reinhardt.

Nauck, Bernhard. 1994. Bildungsverhalten in Migrantenfamilien. In Kindliche Lebenswelten, Bildung und innerfamiliale Beziehungen. Materialien zum Fünften Familienbericht, Band 4, Hrsg. Peter Büchner, Dagmar Meyer, Matthias Grundmann, Sabine Rothe, Johannes Huinink, Lothar Krappmann und Bernhard Nauck, 105-141. Weinheim/München: DJI/Juventa.

Neugebauer, Markus. 2010. Bildungsungleichheit und Grundschulempfehlung beim Übergang auf das Gymnasium. Eine Dekomposition primärer und sekundärer Herkunftseffekte. Zeitschrift für Soziologie 39:202-214.

Neugebauer, Martin, und Steffen Schindler. 2012. Early transitions and tertiary enrolment: The cumulative impact of primary and secondary effects on entering university in Germany. Acta Sociologica 55:19-36.

Oeter, Karl, Jürgen Collatz, Hans Hecker, und Johann-Jürgen Rohde. 1979. Werden die präventiven Möglichkeiten der Schwangerenvorsorge ausreichend genutzt? Erste Ergebnisse der Perinatalstudie Hannover. Gynäkologe 12:164-174.

O’Leary, Colleen M., Natasha Nassar, Jennifer J. Kurinczuk, und Carol Bower. 2009. The effect of maternal alcohol consumption on fetal growth and preterm birth. International Journal of Obstetrics and Gynaecology 116:390-400. 
Ormel, Johan, Siegwart Lindenberg, Nardi Steverink, und Michael Vonkorff. 1997. Quality of life and social production functions: A framework for understanding health effects. Social science \& medicine 45:1051-1063.

Ostner, Ilona, und Carolyn Stolberg. 2015. Investing in Children, Monitoring Parents: Parenting Support in the Changing German Welfare State. Social Policy and Society 14:621632.

Paulus, Wolfgang E. 2006. Medikamente in Schwangerschaft und Stillzeit. In Die Geburtshil$f e$, Hrsg. Henning Schneider, Peter Husslein und Karl-Theo M. Schneider, 81-102. Heidelberg: Springer.

Peacock, Janet L., J. Martin Bland, und H. Ross Anderson. 1995. Preterm delivery: Effects of socioeconomic factors, psychological stress, smoking, alcohol, and caffeine. British Medical Journal 311:531-536.

Peter, Frauke, und C. Katharina Spieß. 2015. Kinder mit Migrationshintergrund in Kindertageseinrichtungen und Horten: Unterschiede zwischen den Gruppen nicht vernachlässigen! DIW Wochenbericht 1+2:12-21.

Petermann, Franz, Kay Niebank, und Herbert Scheithauer. 2000. Risiken in der frühkindlichen Entwicklung. Göttingen: Hogrefe-Verlag.

Peters, Frederik. 2010. Späte Mutterschaft als medizinischer Risikofaktor? Der Einfluss des Alters der Mutter auf das Risiko der Frühgeburt. SOEPpapers Nr. 342. Berlin.

Petrou, Stavros, Tracey Sach, und Leslie Davidson. 2001. The long-term costs of preterm birth and low birth weight: Results of a systematic review. Child: Care, Health and Development 27:97-115.

Pevalin, David J., und David Rose. 2002. Social capital for health: Investigating the links between social capital and health using the British Household Panel Survey. London: Health Development Agency.

Phillips, Kathryn A., Kathleen R. Morrison, Ronald Andersen, und Lu Ann Aday. 1998. Understanding the Context of Healthcare Utilization: Assessing Environmental and Provider-Related Variables in the Behavioral Model of Utilization. Health Services Research 33:571-596.

Ponce, Ninez A., Katherine J. Hoggat, Michelle Wilhelm, und Beate Ritz. 2005. Preterm Birth: The Interaction of Traffic-related Air Pollution with Economic Hardship in Los Angeles Neighborhoods. American Journal of Epidemiology 162:140-148.

Poortinga, Wouter. 2006. Social relations or social capital? Individual and community health effects of bonding social capital. Social Science \& Medicine 63:255-270.

Porter, Travis R., Shia T. Kent, Wei Su, Heidi M. Beck, und Julia M. Gohlke. 2014. Spatiotemporal association between birth outcomes and coke production and steel making facilities in Alabama, USA: a cross-sectional study. Environmental Health 13:1-8.

Portes, Alejandro. 1998. Social capital: Its origins and applications in modern sociology. Annual Review of Sociology 24:1-24. 
Power, Chris, und Diana Kuh. 2008. Die Entwicklung gesundheitlicher Ungleichheiten im Lebenslauf. In Soziale Ungleichheit und Gesundheit: Erklärungsansätze und gesundheitspolitische Folgerungen, Hrsg. Johannes Siegrist und Michael Marmot, 27-53. Bern: Hans Huber.

Putnam, Robert D. 1993. Making Democracy Work: Civic Traditions in Modern Italy. Princeton, NJ: University Press.

Putnam, Robert D. 1995. Bowling alone: America's declining social capital. Journal of Democracy 6:65-78.

Quigley, Maria, Gry Poulsen, Elaine Boyle, Dieter Wolke, David Field, Zarko Alfirevicet, und Jennifer Kurinczuk. 2012. Early term and late preterm birth is associated with poorer school performance at age 5 years: A cohort study. Archive of Disease in Childhood Fetal Neonatal Edition 97:167-173.

Raum, Elke, Birgit Arabin, Martin Schlaud, Ulla Walter, und Friedrich Wilhelm Schwartz. 2001. The impact of maternal education on intrauterine growth: a comparison of former West and East Germany. International Journal of Epidemiology 30:81-87.

Razum, Oliver, Anne Reeske, und Jacob Spallek. 2011. Gesundheit von Schwangeren und Säuglingen mit Migrationshintergrund. Frankfurt am Main: Peter Lang GmbH.

Razum, Oliver, und Hajo Zeeb. 1998. Epidemiologic studies among foreign immigrants in Germany - rational and obstacles. Gesundheitswesen 60:283-286.

Razum, Oliver, Hajo Zeeb, Uta Meesmann, Liane Schenk, Maren Bredehorst, Patrick Brzoska, Tanja Dercks, Susanne Glodny, Björn Menkhaus, Ramazan Salman, Anke C. Saß, und Ralf Ulrich. 2008. Migration und Gesundheit. Schwerpunktbericht der Gesundheitsberichterstaatung des Bundes. Berlin: Robert Koch-Institut.

Reeske, Anne, Marcus Kutschmann, Oliver Razum, und Jacob Spallek. 2011a. Stillbirth differences according to regions of origin: an analysis of the German perinatal database, 2004-2007. BMC Pregnancy and Childbirth 11:1-10.

Reeske, Anne, Jacob Spallek, und Oliver Razum. 2011b. Migration und Gesundheit. In Gesundheit von Schwangeren und Säuglingen mit Migrationshintergrund, Hrsg. Oliver Razum, Anne Reeske und Jacob Spallek, 15-29. Frankfurt am Main: Peter Lang GmbH.

Reeske, Anne, Jacob Spallek, und Oliver Razum. 2009. Changes in smoking prevalence among first and second-generation Turkish migrants in Germany - an analysis of the 2005 Microcensus. International Journal for Equity Health 8:1-9.

Reime, Birgit, Ulrike Lindwedel-Reime, Karin M. Ertl, Carina Jacob, Beate A. Schücking, und Paul Wetzlaff. 2009. Does underutilization of prenatal care explain the excess risk for stillbirth among women with migration background in Germany? Acta Obstetricia et Gynecologica 88:1276-1283.

Reime, Birgit, Pamela A. Ratner, Sandra N. Tomaselli-Reime, Ann Kelly, Beate A. Schuecking, und Paul Wenzlaff. 2006. The role of mediating factors in the association between social deprivation and low birth weight in Germany. Social Science and Medicine 62:1731-1744. 
Reiss, Katharina, Jacob Spallek, und Oliver Razum. 2010. 'Imported risk' or 'health transition'? Smoking prevalence among ethnic German immigrants from the Former Soviet Union by duration of stay in Germany - analysis of microcensus data. International Journal for Equity in Health 9:1-9.

Relikowski, Ilona, Schneider, Thorsten, und Blossfeld, Hans-Peter. 2010. Primäre und sekundäre Herkunftseffekte beim Übergang in das gegliederte Schulsystem: Welche Rolle spielen soziale Klasse und Bildungsstatus in Familien mit Migrationshintergrund? In Komparative empirische Sozialforschung. Anwendungsfelder und aktuelle Methoden in Best Practice-Studien, Hrsg. Tilo Beckers, Klaus W. Birkelbach, Jörg Hagenah und Ulrich Rosar, 143-167. Wiesbaden: VS Verlag für Sozialwissenschaften.

Rettwitz-Volk, Werner. 2003. Mortalität und Morbidität von Frühgeborenen - Eine Zusammenfassung der deutschen Neonatalerhebungen 2001. Zeitschrift für Geburtshilfe und Neonatalogie 207:143-147.

Richter, Matthias. 2005. Gesundheit und Gesundheitsverhalten im Jugendalter. Der Einfluss sozialer Ungleichheit. Wiesbaden: VS Verlag für Sozialwissenschaften.

Richter, Matthias, und Klaus Hurrelmann. 2006. Gesundheitliche Ungleichheit. Grundlagen, Probleme, Perspektiven. Wiesbaden: VS Verlag für Sozialwissenschaften.

Richter, Matthias, und Klaus Hurrelmann. 2007. Warum die gesellschaftlichen Verhältnisse krank machen. Aus Politik und Zeitgeschichte 42:3-10.

Richter, Matthias, und Klaus Hurrelmann. 2009. Gesundheitliche Ungleichheit: Ausgangsfragen und Herausforderungen. In Gesundheitliche Ungleichheit. Grundlagen, Probleme, Perspektiven, Hrsg. Matthias Richter und Klaus Hurrelmann, 13-34. Wiesbaden: VS Verlag für Sozialwissenschaften.

Richter, Matthias, und Klaus Hurrelmann. 2016. Life course influences on health and health inequalities: A socialisation perspective. Zeitschrift für Soziologie der Erziehung und Sozialisation 36:264-280.

Rimbach, Erwin. 1967. Schwangerschaften und Geburten bei Ausländerinnen. Archives of Gynecology and Obstetrics 204:293-295.

Robert Koch-Institut (RKI). 2005. Übergewicht und Adipositas. Berlin: Robert Koch Institut.

Robert Koch-Institut (RKI). 2006. Gesundheit in Deutschland. Gesundheitsberichtserstattung des Bundes. Berlin: Robert Koch Institut.

Robert Koch-Institut (RKI). 2008. Kinder- und Jugendgesundheitssurvey (KiGGS) 20032006: Kinder und Jugendliche mit Migrationshintergrund in Deutschland. Bericht im Auftrag des Bundesministeriums für Gesundheit. Berlin: Robert Koch-Institut.

Rosenbaum, Heidi. 1998. Verwandtschaft in historischer Perspektive. In Verwandtschaft. Sozialwissenschaftliche Beiträge zu einem vernachlässigten Thema, Hrsg. Michael Wagner und Yvonne Schütze, 14-34 Stuttgart: Ferdinand Enke.

von Rosenbladt, Bernhard, SOEP-Gruppe und Infratest Sozialforschung. 2003. SOEP 2003 Methodenbericht zum Befragungsjahr 2003 (Welle 20) des Sozio-oekonomischen Panels. München: Infratest Sozialforschung. 
Rütten, Alfred, Karim Abu-Omar, Thomas Lampert, und Thomas Ziese. 2005. Körperliche Aktivität (Gesundheitsberichtserstattung des Bundes, Heft 26). Berlin: Robert Koch Institut. Rössel, Jörg. 2009. Sozialstrukturanalyse. Eine kompakte Einführung. Wiesbaden: VS Verlag für Sozialwissenschaften.

Rutter, Derek R., und Lyn Quin.1990. Inequality in pregnancy outcome - a review of psychosocial and behavioural mediators, Social Science \& Medicine 30:553-568.

Sackmann, Reinhold, und Matthias Wingens. 2001. Theoretische Konzepte des Lebenslaufs: Übergang, Sequenz und Verlauf. In Strukturen des Lebenslaufs. Übergang - SequenzVerlauf, Hrsg. Reinhold Sackmann und Matthias Wingens, 17-50. Weinheim und München: Juventa Verlag.

Schäfer, Gerd E. 2003. Bildung beginnt mit der Geburt. Förderung von Bildungsprozessen in den ersten sechs Lebensjahren.Weinheim: Beltz-Verlag.

Schenk, Liane. 2007. Migration und Gesundheit - Entwicklung eines Erklärungs- und Analysemodells für epidemiologische Studien. International Journal of Public Health 52:8796.

Schenk, Liane, Ute Ellert, und Hannelore Neuhauser. 2007. Kinder und Jugendliche mit Migrationshintergrund in Deutschland. Methodische Aspekte im Kinder- und Jugendgesundheitssurvey (KiGGS). Bundesgesundheitsblatt - Gesundheitsforschung - Gesundheitsschutz 50:590-599.

Scheppers, Emmanuel, Els van Dongen, Jos Dekker, Jan Geertzen, und Joost Dekker. 2006. Potential barriers to the use of health services among ethnic minorities: a review. Family Practice 23:325-348.

Schier, Michaela, Karin Jurzyk, und Peggy Szymenderski. 2011. Entgrenzung von Arbeit und Familie - mehr als Prekarisierung. WSI Mitteilungen 8:402-408.

Schlesiger, Claudia, Jennifer Lorenz, Sabine Weinert, Thorsten Schneider, und Hans-Günther Roßbach. 2011. From birth to early child care. Zeitschrift für Erziehungswissenschaft 14:187-202.

Schneider, Thorsten. 2016. Geburten aus geplanten und ungeplanten Schwangerschaften Die Relevanz von Bildung, Arbeitslosigkeit und Partnerschaft. Kölner Zeitschrift für Soziologie und Sozialpsychologie 68:1-28.

Schroedter, Julia H., und Frank Kalter. 2008. Binationale Ehen in Deutschland. Trends und Mechanismen der sozialen Assimilation. In Migration und Integration. Sonderheft 48 der Kölner Zeitschrift für Soziologie und Sozialpsychologie, Hrsg. Frank Kalter, 351379.Wiesbaden: VS Verlag für Sozialwissenschaften.

Schröder, Carsten, C. Katharina Spieß, und Johanna Storck. 2015. Private Bildungsausgaben für Kinder: Einkommensschwache Familien sind relativ stärker belastet. DIW Wochenbericht 8:158-169.

Schultz, Theodore W. 1961. Investment in Human Capital. The American Economic Review 51:1-17. 
Schupp, Jürgen, Sebastian Frischholz, und Christian Schmitt. 2010. 14. BIOAGE01: Generated variables from the ,mother and child questionaire'. In Biography and life history data in the German Socio-Economic Panel (SOEP, v26, 1984-2009), Hrsg. Joachim R. Frick und Henning Lohmann, 163-169. Berlin: Deutsches Institut für Wirtschaftsforschung.

Schwingel, Markus. 1995. Pierre Bourdieu zur Einführung. Hamburg: Junius.

Scriba, Peter C. 2007. Die Ergebnisse des Kinder- und Jugendgesundheitssurveys (KiGGS) eine einzigartige Datensammlung zur Gesundheit der heranwachsenden Bevölkerung in Deutschland. Bundesgesundheitsblatt - Gesundheitsforschung - Gesundheitsschutz 50:531-532.

Shah, Prakesh S. 2011. On behalf of the knowledge synthesis group on determinants of preterm/low birthweight births. Paternal factors and low birthweight, preterm, and small for gestational age births: a systematic review. American Journal of Obstetrics and Gynecology 202:103-123.

Shah, Prakesh S, Jamie Zao, und Samana Ali, Knowledge Synthesis Group of Determinants of preterm/LBW births. 2011. Maternal marital status and birth outcomes: A systematic review and meta-analyses. Maternal and Child Health Journal 7:1097-1109.

Shiono, Patricia H., und Mark A. Klebanoff. 1986. Ethnic Differences in Preterm and Very Preterm Delivery. American Journal of Public Health 76:1317-1321.

Shiono, Patricia H., Virginia A. Rauh, Mikyung Park, Sally A. Lederman, und Deborah Zuskar. 1997. Ethnic Differences in Birthweight: The Role of Lifestyle and Other Factors. American Journal of Public Health 87:787-793.

Siegrist, Johannes. 1995. Medizinische Soziologie. München/Wien/Baltimore: Urban \& Schwarzenberg.

Siegrist, Johannes. 2005. Medizinische Soziologie. München: Elsevier GmbH.

Siegrist, Johannes, und Michael Marmot. 2008. Soziale Ungleichheit und Gesundheit: Erklärungsansätze und gesundheitspolitische Forderungen. Bern: Verlag Hans Huber.

Simoes, Elisabeth, Siegfried Kunz, Margarete Bosing-Schwenkglenks, Peter Schwoerer, und Friedrich Wilhelm Schmahl. 2003. Inanspruchnahme der Schwangerenvorsorge - Ein Spiegel gesellschaftlicher Entwicklungen und Aspekte der Effizienz. Geburtshilfe und Frauenheilkunde 63:538-545.

Skopek, Jan, Florian Schulz, und Hans-Peter Blossfeld. 2009. Partnersuche im Internet. Bildungsspezifische Mechanismen bei der Wahl von Kontaktpartnern. Kölner Zeitschrift für Soziologie und Sozialpsychologie 61:1-28.

Snelgrove, John W., Hynek Pikhart, und Mai Stafford. 2009. A multilevel analysis of social capital and self-rated health: evidence from the British Household Panel Survey. Social Science \& Medicine 68:1993-2001.

SOEP Group. 2001. The German Socio-Economic Panel (SOEP) after more than 15 years An overview. Vierteljahreshefte zur Wirtschaftsforschung 70:7-14. 
Söhn, Janina. 2008. Bildungsunterschiede zwischen Migrantengruppen in Deutschland: Schulabschlüsse von Aussiedlern und anderen Migranten der ersten Generation im Vergleich. Berliner Journal für Soziologie 18:401-431.

Solga, Heike. 1997. Bildungschancen in der DDR. In Bildungsgeschichte einer Diktatur. Bildung und Erziehung in der SBZ und der DDR im historisch-gesellschaftlichen Kontext, Hrsg. Sonja Häder, Heinz-Elmar Tenort, 275-294. Weinheim: Deutscher Studien Verlag.

Solga, Heike. 2005. Meritokratie - die moderne Legitimation ungleicher Bildungschancen. In Institutionalisierte Ungleichheiten. Wie das Bildungswesen Chancen blockiert, Hrsg. Peter A. Berger und Heike Kahlert, 19-38. Weinheim und München: Juventa Verlag.

Solga, Heike, und Rolf Becker. 2012. Soziologische Bildungsforschung - eine kritische Bestandsaufnahme. In Soziologische Bildungsforschung, Kölner Sonderheft 52, Zeitschrift für Soziologie und Sozialpsychologie, Hrsg. Rolf Becker und Heike Solga, 7-43. Wiesbaden: VS Verlag für Sozialwissenschaften.

Solga, Heike, Peter A. Berger, und Justin Powell. 2009. Soziale Ungleichheit - Kein Schnee von gestern! Eine Einführung. In Soziale Ungleichheit. Klassische Texte zur Sozialstrukturanalyse, Hrsg. Heike Solga, Peter A. Berger und Justin Powell, 11-45. Frankfurt a.M.: Campus.

Solga, Heike, und Sandra Wagner. 2008. Die Zurückgelassenen - die soziale Verarmung der Lernumwelt von Hauptschülerinnen und Hauptschülern. In Bildung als Privileg, 3. Auflage, Hrsg. Rolf Becker und Wolfgang Lauterbach, 191-219. Wiesbaden: VS Verlag für Sozialwissenschaften.

Spallek, Jacob, Jessica Lehnhardt, Anna Reeske, Oliver Razum, und Matthias David. 2014. Perinatal outcomes of immigrant women of Turkish, Middle Eastern and North African origin in Berlin, Germany: A comparison of two time periods. Archives of Gynecology and Obstetrics 289:505-512.

Spallek, Jacob, Hajo Zeeb, und Oliver Razum. 2010. Prevention among immigrants: the example of Germany. BMC Public Health 10:1-6.

Sperlich, Stefanie, Kristin Illiger, und Siegfried Geyer. 2011. Warum rauchen Mütter? Analyse des Einflusses der Lebenslage und psychischer Faktoren auf den Tabakkonsum von Müttern minderjähriger Kinder. Bundesgesundheitsblatt 54:1211-1220.

Sperlich, Stefanie, und Andreas Mielck. 2003. Sozialepidemiologische Erklärungsansätze im Spannungsfeld zwischen Schicht- und Lebensstilkonzeptionen. Plädoyer für eine integrative Betrachtung auf der Grundlage der Bourdieuschen Habitustheorie. Zeitschrift für Gesundheitswissenschaften 11:165-179.

Spieß, Christa K. 2013. Investitionen in Bildung: Frühkindlicher Bereich hat großes Potential. DIW Wochenbericht Nr. 26:40-48.

Spieß, Martin. 2010. Der Umgang mit fehlenden Werten. In Handbuch der sozialwissenschaftlichen Datenanalyse, Hrsg. Christof Wolf und Henning Best, 117-142. Wiesbaden: VS Verlag für Sozialwissenschaften. 
Stanat, Petra, Knut Schwippert, und Carola Gröhlich. 2010. Der Einfluss des Migrantenanteils in Schulklassen auf den Kompetenzerwerb: Längsschnittliche Überprüfung eines umstrittenen Effekts. In Migration, Sprache, Identität und Bildungserfolg. 55. Beiheft der Zeitschrift für Pädagogik, Hrsg. Christina Allemann-Ghionda, Petra Stanat, Kerstin Göbel und Charlotte Röhner, 147-164. Weinheim: Beltz.

StataCorp. 2009. Stata Multiple-Imputation Reference Manual. Release 11. Statistical Software. College Station: StataCorp LP.

Statistisches Bundesamt. 2010. Bevölkerung und Erwerbstätigkeit. Haushalte und Familien. Ergebnisse des Mikrozensus 2009. Wiesbaden: Statistisches Bundesamt.

Steinhausen, Hans-Christoph. 2000. Pränatale Entwicklungsgefährdungen - Ergebnisse der Verhaltensteratologie. In Risiken in der frühkindlichen Entwicklung, Hrsg. Franz Petermann, Kay Niebank und Herbert Scheithauer, 101-112. Göttingen: Hogrefe-Verlag.

Sterne, Jonathan A., Ian R. White, John B. Carlin, Michael Spratt, Patrick Royston, Michael G. Kenward, Angela M. Wood, und James R. Carpenter. 2009. Multiple imputation for missing data in epidemiological and clinical research: Potential and pitfalls. British Medical Journal 2009:b2393.

Steyn, Krisela, Thea De Wet, Yussuf Saloojee, Hannelie Nel, und Derek Yach. 2006. The influence of maternal cigarette smoking, snuff use and passive smoking on pregnancy outcomes: the Birth To Ten Study. Paediatric and Perinatal Epidemiology 20:90-99.

Stich, P. H. Heribert Ludwig, Rafael Mikolajczyk, und Alexander Krämer. 2009. Determinanten des Teilnahmeverhaltens bei Kindervorsorgeuntersuchungen (U1 - U8). Eine gesundheitswissenschaftlicher Analyse zur Gesundheitsversorgung im Kindesalter. Prävention und Gesundheitsförderung 4:265-271.

Stocké, Volker. 2012. Das Rational-Choice Paradigma in der Bildungssoziologie. In Handbuch Bildungs- und Erziehungssoziologie, Hrsg. Ullrich Bauer, Uwe H. Bittlingmayer und Alber Scherr, 423-436. Wiesbaden: VS Verlag für Sozialwissenschaften.

Stolberg, Carolyn. 2017. Converging Interventions? Social Investment Elements in Child Health Care Strategies in Germany, Sweden and the United Kingdom. In Parents in the Spotlight. Parenting Practices and Support from a Comparative Perspective (Journal of Family Research, Special Issue 11), Hrsg. Tanja Betz, Michael-Sebastion Honig und Ilona Ostner, 293-312. Opladen: Barbara Budrich Verlag.

Stolberg, Carolyn, und Sten Becker. 2015. Gesundheitliche Ungleichheit zum Lebensbeginn. Zum Einfluss der mütterlichen Bildung auf die Wahrscheinlichkeit einer Frühgeburt. Kölner Zeitschrift für Soziologie und Sozialpsychologie 67:321-354.

Strandberg-Larsen, Katrine, Naja Rod Nielsen, Anne-Marie Nybo Andersen, Jørn Olsen, und Morten Grønbæk. 2008. Characteristics of women who binge drink before and after they become aware of their pregnancy. European Journal of Epidemiology 23:565-572.

Straube, Sebastian, Manfred Voigt, Rembrandt D. Scholz, Frederik Peters, Ernst Hallier, Volker Briese, und Gerhard Jorch. 2009. 18th Communication: Preterm birth rates and ma- 
ternal occupation - the importance of age and number of live births as confounding factors. Geburtshilfe und Frauenheilkunde 69:698-702.

Strobel, Bernadette, und Cornelia Kristen. 2015. Erhalt der Herkunftssprache? - Muster des Sprachgebrauchs in Migrantenfamilien. Zeitschrift für Erziehungswissenschaft 18:125142.

Sucksdorff, Minna, Liisa Lehtonen, Roshan Chudal, Auli Suominen, Petteri Joelsson, Mika Gissler, und Andre Sourander. 2015. Preterm Birth and Poor Fetal Growth as Risk Factors of Attention-Deficit/ Hyperactivity Disorder. Pediatrics 136:e599-608.

Sullivan, Alice. 2001. Cultural capital and educational attainment. Sociology 35:893-912.

Svensson, Anna C., Sven Sandin, Sven Cnattingius, Marie Reilly, Yudi Pawitan, Christina M. Hultman, und Paul Lichtenstein. 2009. Maternal effects for preterm birth: A genetic epidemiologic study of 630,000 Families. American Journal of Epidemiology 170:13651372 .

Tamm, Marcus. 2005. The effect of poverty on the health of newborn children. Evidence from Germany. RWI Discussion Papers 33. Essen.

Teitler, Julien O. 2001. Father involvement, child health, and maternal health behavior. Children and Youth Services Revue 23:403-425.

Thaiss, Heidrun, Renate Klein, Eike C. Schumann, Gabriele Ellsäßer, Helmut Breitkopf, Heike Reinecke, und Eberhard Zimmermann. 2010. Früherkennungsuntersuchungen als Instrument im Kinderschutz. Bundesgesundheitsblatt - Gesundheitsforschung - Gesundheitsschutz, 53:1029-1047.

Tietze, Konrad W., Eike Bartholomeyczik, Sabine Bartholomeyczik, Petra Jaedicke, Ursula Jaensch, und Heidi Trull. 1982. Epidemiologische und sozialmedizinische Aspekte der Schwangerschaft. Eine Untersuchung zu den sozialen regionalen Bedingungen von Schwangerenvorsorge. Der Bundesminister für Arbeit und Sozialordnung. Gesundheitsforschung Bd. 70. Bonn.

TNS Infratest Sozialforschung. 2015a. SOEP 2015 - Erhebungsinstrumente 2015 (Welle 32) des Sozio-oekonomischen Panels: Eltern und Kind (7-8 Jahre), Altstichproben. SOEP Survey Papers 280: Series. Berlin: DIW/SOEP.

Troschke, Jürgen von. 2006. Gesundheits- und Krankheitsverhalten. In Handbuch Gesundheitswissenschaften, Hrsg. Klaus Hurrelmann, Ulrich Laaser, und Oliver Razum, 529560. Weinheim: Juventa.

Tuppat, Julia, und Birgit Becker. 2014. Sind türkischstämmige Kinder beim Schulstart im Nachteil? Die Bedeutung genereller und aufnahmelandspezifischer Kompetenzen für die Wahrscheinlichkeit einer Rückstellung. Kölner Zeitschrift für Soziologie und Sozialpsychologie 66:219-241.

Turner, Ralph H. 1960. Sponsored and contest mobility and the school system. American Sociological Review 25:855-867.

Unger, Rainer. 2008. Gesundheit im Lebenslauf. Zur relativen Bedeutung von Selektionsgegenüber Kausaleffekten am Beispiel des Familienstands. In Health Inequalities. De- 
terminanten und Mechanismen gesundheitlicher Ungleichheit, Gesundheit und Gesellschaft, Hrsg. Ulrich Bauer, Uwe Bittlingmeyer und Matthias Richter, 430-451. Wiesbaden: VS Verlag für Sozialwissenschaften.

Urech, Corinne, Sandra Scherer, und Judith Alder. 2012. Stressfaktor drohende Frühgeburt. Psychoscope 5:4-7.

Vallet, Louis-André, und Jean-Paul Caille. 1999. Migration and Integration in France. Academic Careers of Immigrants' Children in Lower and Upper Secondary School. Paper prepared for the ESF Conference "Migrations and Inter-Ethnic Relations in Europe" Obernai, September 1999, 23-28.

Verkerk, Paul H., Boukje M. Zaadstra, J. Dorothea Reerink, W. Pieter Herngreen, und S. Pauline Verloove-Vanhorick. 1994. Social class, ethnicity and other risk factors for small for gestational age and preterm delivery in the Netherlands. European Journal of Obstetrics \& Gynecology and Reproductive Biology 53:129-134.

Villa, Paula-Irene. 2008. Körper. In Handbuch Soziologie, Hrsg. Nina Bauer, Hermann Korte, Martina Löw und Markus Schroer, 201-217. Wiesbaden: VS Verlag für Sozialwissenschaften.

Voigt, Manfred, Volker Briese, Vera Pietzner, Sylvia Kirchengast, Klaus-Theo M. Schneider, Sebastian Straube, und Gerhard Jorch. 2009. Evaluierung von mütterlichen Merkmalen als Risikofaktoren für Frühgeburtlichkeit (Einzel- und Kombinationswirkung). Zeitschrift für Geburtshilfe und Neonatalogie 213:138-146.

Voigt, Manfred, Christoph Fusch, Dirk Olbertz, Martin H. Carstensen, Andreas Brüning, Klaus Hartmann, und Volker Hesse. 2006b. 10. Mitteilung: Zum Anstieg der SGANeugeborenenrate durch das Rauchen der Mütter in der Schwangerschaft und Risikostruktur von „starken Raucherinnen“ (Analyse des Geburtenkollektivs der Jahre 19951997 der Bundesrepublik Deutschland). Geburtshilfe und Frauenheilkunde 66:270-276.

Voigt, Manfred, Klaus-Theo M. Schneider, H. Brinks, Christoph Fusch, Klaus Hartmann, Ursula Wittwer-Backofen, und Volker Hesse. 2006a. 11. Mitteilung: Unterschiede im somatischen Entwicklungsstand Neugeborener unter Berücksichtigung des Herkunftslandes der Mütter (Analyse des Neugeborenenkollektivs der Jahre 1995-1997 der Bundesrepublik Deutschland). Geburtshilfe und Frauenheilkunde 66:391-399.

Voigt, Manfred, Sebastian Straube, Christoph Fusch, Guido Heineck, Dirk M. Olbertz, und Klaus-Theo M. Schneider. 2007. Erhöhung der Frühgeborenenrate durch Rauchen in der Schwangerschaft und daraus resultierende Kosten für die Perinatalmedizin in Deutschland. Zeitschrift für Geburtshilfe und Neonatologie 211:204-210.

Wagner, Gert G., Joachim R. Frick, und Jürgen Schupp. 2007. The German Socio-Economic Panel Study (SOEP) - Scope, evolution and enhancements. Schmollers Jahrbuch - Journal of Applied Social Science Studies, 121:139-169.

Wagner, Michael, und Yvonne Schütze. 1998. Verwandtschaft. Sozialwissenschaftliche Beiträge zu einem vernachlässigten Thema. Stuttgart: Ferdinand Enke. 
Walker, Meghan J., Ban Al-Sahab, Farah Islam, und Hala Tamim. 2011. The epidemiology of alcohol utilization during pregnancy: An analysis of the Canadian Maternity Experiences Survey (MES). BMC Pregnancy and Childbirth 11:1-9.

Weber, Ingbert. 1994. Soziale Schichtung und Gesundheit. In Soziale Schichten und Lebenschancen in Deutschland, Hrsg. Rainer Geißler, 195-219. Stuttgart: Ferdinand Enke Verlag.

Weber, Max. 1972. Wirtschaft und Gesellschaft: Grundriss der verstehenden Soziologie. Tübingen: Mohr Siebeck.

Wendt, Claus, und Christoph Wolf. 2006. Soziologie der Gesundheit. Sonderband 46 der Kölner Zeitschrift für Soziologie und Sozialpsychologie. Wiesbaden: VS Verlag für Sozialwissenschaften.

von der Wense, Axel, und Carola Bindt. 2013. Risikofaktor Frühgeburt. Entwicklungsrisiken erkennen und behandeln. Weinheim und Basel: Beltz Verlag.

van de Werfhorst, Herman G., und Frank Van Tubergen. 2007. Ethnicity, Schooling, and Merit in the Netherlands. Ethnicities 7:416-444.

Werning, Rolf. 2014. Stichwort: Schulische Inklusion. Zeitschrift für Erziehungswissenschaft 17:601-623.

Weyers, Simone. 2008. Soziale Ungleichheit, soziale Beziehungen und Gesundheitsverhalten. In Health Inequalities. Determinanten und Mechansimen gesundheitlicher Ungleichheit, Hrsg. Ulrich Bauer, Uwe H. Bittlingmayer, und Matthias Richter, 257-270. Wiesbaden: VS Verlag für Sozialwissenschaften.

White, Ian R., Patrick Royston, und Angela M. Wood. 2011. Multiple imputation using chained equations: Issues and guidance for practice. Statistics in Medicine 30:377-399.

Williams, Redford B. 1998. Lower socioeconomic status and increased mortality: early childhood roots and the potential for successful interventions. Journal of the American Medical Association 279:1745-1746.

Williams, Simon J. 1995. Theorising class, health and lifestyles: Can Bourdieu help us? Sociology of Health and Illness 17:577-604.

Winkler, Oliver. 2017. Aufstiege und Abstiege im Bildungsverlauf. Eine empirische Untersuchung zur Öffnung von Bildungswegen. Wiesbaden: VS Verlag für Sozialwissenschaften.

Winship, Christopher, und Robert D. Mare. 1984. Regression models with ordinal variables. American Sociological Review 49:512-525.

Wolf, Christof. 2006. Psychosozialer Stress und Gesundheit. Belastungen durch Erwerbsarbeit, Hausarbeit und soziale Beziehungen. In Soziologie der Gesundheit. Sonderband 46 der Kölner Zeitschrift für Soziologie und Sozialpsychologie, Hrsg. Claus Wendt und Christof Wolf, 86-108. Wiesbaden: VS Verlag für Sozialwissenschaften.

Wolf, Christof, und Henning Best. 2010. Logistische Regressionen. In Handbuch der sozialwissenschaftlichen Datenanalyse, Hrsg. Christof Wolf und Henning Best, 827-854. Wiesbaden: VS Verlag für Sozialwissenschaften. 
Wolke, Dieter, und Renate Meyer. 1999. Cognitive status, language attainment, and prereading skills of 6-year-old very preterm children and their peers: The Bavarian longitudinal study. Developmental Medicine \& Child Neurology 41:94-109.

Wolke, Dieter, und Renate Meyer. 2000. Ergebnisse der Bayerischen Entwicklungsstudie an neonatalen Risikokindern: Implikationen für Theorie und Praxis. In Risiken in der frühkindlichen Entwicklung - Entwicklungspathologie der ersten Lebensjahre, Hrsg. Franz Petermann, Kay Niebank und Herbert Scheithauer, 113-138. Göttingen: Hogrefe-Verlag.

Wood, Angela M, Ian R. White, und Patrick Royston. 2008. How should variable selection be performed with multiply imputed data? Statistics in Medicine 27:3277-3246.

Woodruff, Tracey J., Jennifer D. Parker, Amy D. Kyle, und Kenneth C. Schoendorf. 2003. Disparities in Exposure to Air Pollution during Pregnancy. Environmental Health Perspectives 111:942-946.

World Health Organization (WHO). 1986. Ottawa-Charta zur Gesundheitsförderung. Die Ortskrankenkasse 70:117-120.

World Health Organization (WHO). 1998. The world health report 1998. Life in the 21 st century: A vision for all. Switzerland: WHO Publications.

World Health Organization (WHO). 2005. The world health report 2005. Make every mother and child count. Switzerland: WHO Publications.

World Health Organization, und United Children's Fund (WHO und UNICEF). 2004. Low birthweight. Country, regional and global estimates. Switzerland: WHO Publications.

Wulff, Karl-Heinrich. 1997. Frühgeburt und Grenzen. Der Gynäkologe 30:539-543.

Xie, Yiqiong, Emily Wheeler Harville, und Aubrey Spriggs Madkour. 2014. Academic performance, educational aspiration and birth outcomes among adolescent mothers: A national longitudinal study. BMC Pregnancy and Childbirth 14:1-11. 


\section{Eidesstattliche Erklärung}

„Ich versichere, dass ich die eingereichte Dissertation „Ursachen früher gesundheitlicher Ungleichheiten und ihre schulischen Folgen" selbstständig und ohne unerlaubte Hilfsmittel verfasst habe. Anderer als der von mir angegebenen Hilfsmittel und Schriften habe ich mich nicht bedient. Alle wörtlich oder sinngemäß den Schriften anderer Autoren entnommenen Stellen habe ich kenntlich gemacht.“ 